\title{
Österreichischer Blutdruckkonsens 2019
}

\author{
Thomas Weber · Klaus Arbeiter · Florian Ardelt - Johann Auer · Christoph Aufricht · Mathias C. Brandt · \\ Wolfgang Dichtl · Julia Ferrari · Bernhard Föger · Martin Henkel · Katharina Hohenstein-Scheibenecker · \\ Sabine Horn · Alexandra Kautzky-Willer · Erwin Kepplinger · Michael Knoflach · Christian Koppelstätter · \\ Christoph Mache - Peter Marschang · Gert Mayer - Bernhard Metzler - Rainer Oberbauer · Florian Obermair · \\ Barbara Obermayer-Pietsch · Sabine Perl · Stefan Pilz · Friedrich C. Prischl · Andrea Podczeck-Schweighofer · \\ Erwin Rebhandl · Miklos Rohla · Regina Roller-Wirnsberger · Christoph H. Saely · Peter Siostrzonek · Jörg Slany • \\ Kurt Stoschitzky · Siegfried Waldegger · Rene R. Wenzel · Thomas Weiss · Gerhard Wirnsberger • \\ Yvonne Winhofer-Stöckl · David Zweiker · Robert Zweiker · Bruno Watschinger · Österreichische Gesellschaft für \\ Hypertensiologie - Österreichische Atherosklerosegesellschaft · Österreichische Diabetes Gesellschaft . \\ Österreichische Gesellschaft für Internistische Angiologie · Österreichische Gesellschaft für Nephrologie • \\ Österreichische Kardiologische Gesellschaft • Österreichische Gesellschaft für Neurologie · Österreichische \\ Schlaganfall-Gesellschaft · Österr. Gesellschaft für Allgemeinmedizin · Österr. Gesellschaft für Geriatrie • \\ Österreichische Gesellschaft für Endokrinologie und Stoffwechsel · Österreichische Gesellschaft für Innere \\ Medizin · Österreichische Gesellschaft für Kinder- und Jugendheilkunde
}

Zusammenfassung Erhöhter Blutdruck bleibt eine Hauptursache von kardiovaskulären Erkrankungen, Behinderung und frühzeitiger Sterblichkeit in Österreich, wobei die Raten an Diagnose, Behandlung und Kontrolle auch in rezenten Studien suboptimal sind. Das Management von Bluthochdruck ist eine häufige Herausforderung für Ärztinnen und Ärzte vieler Fachrichtungen. In einem Versuch, diagnostische und therapeutische Strategien zu standardisieren und letztendlich die Rate an gut kontrollierten Hypertoniker/innen zu erhöhen und dadurch kardiovaskuläre Erkrankungen zu verhindern, haben 13 österreichische medizinische Fachgesellschaften die vorhandene Evidenz zur Prävention, Diagnose, Abklärung, Therapie und Konsequenzen erhöhten Blutdrucks gesichtet. Das hier vorgestellte Ergebnis ist der erste Österreichische Blutdruckkonsens. Die Autoren und die beteiligten Fachgesellschaften sind davon überzeugt, daß es einer gemeinsamen nationalen Anstrengung bedarf, die Blutdruck-assoziierte Morbidität und Mortalität in unserem Land zu verringern.

Schlüsselwörter Arterielle Hypertonie - Guidelines · Blutdruck · Therapie

\section{Austrian Consensus on High Blood Pressure 2019}

Summary Elevated blood pressure remains a major cause of cardiovascular disease, disability, and pre- mature death in Austria, with suboptimal rates of detection, treatment and control also in recent years. Management of hypertension is a common challenge for physicians with different spezializations. In an attempt to standardize diagnostic and therapeutic strategies and, ultimately, to increase the rate of patients with controlled blood pressure and to decrease the burden of cardiovascular disease, 13 Austrian medical societies reviewed the evidence regarding prevention, detection, workup, treatment and consequences of high blood pressure in general and in various clinical scenarios. The result is presented as the first national consensus on blood pressure. The authors and societies involved are convinced that a joint national effort is needed to decrease hypertension-related morbidity and mortality in our country.

Keywords Arterial Hypertension · Guidelines · Blood Pressure · Treatment

Koordination: Thomas Weber

PD Dr. T. Weber $(\bowtie)$

Abteilung für Innere Medizin II - Kardiologie, Intensivmedizin, Klinikum Wels-Grieskirchen, Grieskirchnerstraße 42, 4600 Wels, Österreich thomas.weber3@liwest.at 


\section{K. Arbeiter $\cdot$ C. Aufricht}

Universitätsklinik für Kinder- und Jugendheilkunde, Abteilung Pädiatrische Nephrologie und Gastroenterologie, Medizinische Universität Wien, Wien, Österreich

F. Ardelt

Gemeinschaftspraxis für Allgemeinmedizin, Marchtrenk, Österreich

J. Auer

Interne Abteilung, Krankenhaus Sankt Josef Braunau, Braunau, Österreich

M. C. Brandt

Klinik II für Innere Medizin, Universitätsklinikum Salzburg, Müllner Hauptstraße 48, 5020 Salzburg, Österreich

W. Dichtl · B. Metzler

Universitätsklinik für Innere Medizin III - Kardiologie und Angiologie, Medizinische Universität Innsbruck,

Anichstraße 35, 6020 Innsbruck, Österreich

J. Ferrari

Abteilung für Neurologie, neurologische Rehabilitation und Akutgeriatrie, Krankenhaus der Barmherzigen Brüder Wien, Wien, Österreich

\section{B. Föger}

Abteilung für Innere Medizin, Krankenhaus Pfarrkirchen, Pfarrkirchen, Österreich

M. Henkel

Abteilung für Kinder- und Jugendheilkunde,

Ordensklinikum Linz Barmherzige Schwestern, Linz,

Österreich

K. Hohenstein-Scheibenecker

Pflegewohnhaus Donaustadt, Sozialmedizinisches Zentrum Ost - Wiener Krankenanstaltenverbund, Langobardenstaße 122a, 1220 Wien, Österreich

\section{S. Horn}

Abteilung für Innere Medizin, Landeskrankenhaus Villach, Villach, Österreich

\section{A. Kautzky-Willer · Y. Winhofer-Stöckl}

Klinik für Innere Medizin III, Abteilung für Endokrinologie und Stoffwechsel, Medizinische Universität Wien, Wien,

Österreich

\section{E. Kepplinger}

Gemeinschaftspraxis für Allgemeinmedizin Leonding, Leonding, Österreich

\section{Knoflach}

Universitätsklinik für Neurologie, Medizinische Universität Innsbruck, Anichstraße 35, 6020 Innsbruck, Österreich

\section{Koppelstätter $\cdot$ G. Mayer}

Universitätsklinik für Innere Medizin IV, Medizinische Universität Innsbruck, Anichstraße 35, 6020 Innsbruck, Österreich

\section{Mache}

Universitätklinik für Kinder- und Jugendheilkunde,

Klinische Abteilung für Allgemeine Pädiatrie, Medizinische Universität Graz, Graz, Österreich

P. Marschang

Abteilung für Innere Medizin, Zentralkrankenhaus Bozen, Lorenz-Böhler-Straße 5, 39100 Bozen, Italien

\section{R. Oberbauer $\cdot$ B. Watschinger}

Klinik für Innere Medizin III, Abteilung für Nephrologie und Dialyse, Medical University of Vienna, Währinger Gürtel 18-20, 1090 Wien, Österreich

F. Obermair

Praxis für Allgemeinmedizin, Eberschwang, Österreich

B. Obermayer-Pietsch · S. Pilz

Universitätsklinik für Innere Medizin, Klinische Abteilung für Endokrinologie und Diabetologie, Medizinische Universität Graz, Graz, Österreich

S. Perl $\cdot$ K. Stoschitzky $\cdot$ R. Zweiker

Abteilung für Kardiologie, Medizinische Universität Graz, Auenbruggerplatz 15, 8036 Graz, Österreich

F. C. Prischl

4. Interne Abteilung, Bereich Nephrologie, Klinikum

Wels-Grieskirchen, 4600 Wels, Österreich

A. Podczeck-Schweighofer

5. Medizinische Abteilung/Kardiologie, Kaiser Franz Josef-Spital, Wien, Österreich

E. Rebhandl

Primärversorgungszentrum Haslach, Haslach an der Mühl, Österreich

M. Rohla $\cdot$ D. Zweiker

3. Medizinische Abteilung, Kardiologie und internistische Intensivmedizin, Wilhelminenspital, Wien, Österreich

\section{R. Roller-Wirnsberger}

Universitätsklinik für Innere Medizin, Gemeinsame

Einrichtung, Medizinische Universität Graz,

Auenbruggerplatz 15, $8036 \mathrm{Graz}$, Österreich

C. H. Saely

Landeskrankenhaus Feldkirch, Akademisches

Lehrkrankenhaus, 6800 Feldkirch, Österreich

VIVIT Institut, Feldkirch, Österreich

Private Universität im Fürstentum Liechtenstein (UFL), Triesen, Liechtenstein

P. Siostrzonek

Interne II - Kardiologie, Ordensklinikum Linz Barmherzige Schwestern, Seilerstätte 4, 4060 Linz, Österreich

J. Slany

Promenadengasse 21/3, 1170 Wien, Österreich

S. Waldegger

Tirolkliniken, Pädiatrie I, Medizinische Universität

Innsbruck, Innsbruck, Österreich

R. R. Wenzel

Interne Abteilung, A.ö. Tauernkliniken - Zell am See \& Mittersill, Paracelsusstr. 8, 5700 Zell am See, Österreich

T. Weiss

Privatklinik Döbling, Wien, Österreich

Karl Landsteiner Institut für Kardiometabolik, St. Pölten, Österreich

Lehrstuhl für experimentelle Kardiologie, Sigmund Freud PrivatUniversität Wien, Wien, Österreich

\section{G. Wirnsberger}

Medizinische Universität Graz, Klinische Abteilung für Nephrologie, Universitätsklinik für Innere Medizin, Auenbruggerplatz 27, 8036 Graz, Österreich 


\section{Kapitelübersicht}

1. Präambel

2. Die Situation in Österreich

3. Diagnose der Hypertonie - Blutdruckmessung

4. Erweiterte Diagnose und Risikoeinschätzung

5. Basisabklärung bei Hypertonie

6. Hypertonie-vermittelte Organschäden

7. Sekundäre Hypertonie

8. Hypertonie und Nierenarterienstenose

9. Endokrine Hypertonie

10. Nichtmedikamentöse Blutdrucksenkung

11. Blutdrucksenkende Medikamente

12. Blutdruckzielwerte bei unkomplizierter Hypertonie

13. Blutdruck und Herzerkrankungen

14. Blutdruck und vaskuläre Erkrankungen

15. Blutdruck und Diabetes mellitus

16. Blutdruck und Nierenerkrankungen

17. Blutdruck und neurologische Erkrankungen

18. Blutdruck während und nach der Schwangerschaft

19. Blutdruck bei Kindern und Jugendlichen

20. Blutdruck im Alter

21. Hypertensiver Notfall - hypertensive Krise

22. Blutdruckmanagement perioperativ

23. Resistente Hypertonie

24. Interventionelle Verfahren zur Blutdrucksenkung

25. Hypertonie und Adhärenz

26. Gesprächsführung

27. Welche Medikamente sollten vermieden werden?

28. Blutdruckschulung und Empowerment

29. Kontrollen und Kontrollintervalle

\section{Präambel}

Die vorliegenden Empfehlungen sollen behandelnden Ärztinnen und Ärzten auf Basis der verfügbaren Evidenz eine Unterstützung für die Diagnostik und Therapie von Patient/innen mit Bluthochdruck geben. Guidelines und Empfehlungen sind allgemeiner Natur und sind in den meisten Situationen für die meisten Patient/innen zutreffend. Die Empfehlungen richten sich explizit an Ärztinnen und Ärzte, da diese aufgrund ihrer medizinischen Ausbildung in der Lage sind, auch individuell sinnvolle und notwendige Abweichungen von den Empfehlungen vorzunehmen, wenn diese aufgrund einer speziellen Situation (Verfügbarkeit der Mittel, Restriktionen anderer Art, individueller Patient/innenwille/-bedürfnis etc.) erforderlich sind. Ärztinnen und Ärzte, die sich auf die Empfehlungen beziehen, sind dafür verantwortlich, die Sinnhaftigkeit, Anwendbarkeit sowie deren Umsetzung im jeweiligen Einzelfall zu überprüfen. Die Empfehlungen richten sich primär nicht an andere Berufsgruppen und sind in ihrer Art daher nicht ohne Weiteres bzw. ohne vorherige Diskussion als Grundlage für Entscheidungen anderer Fachgruppen, z.B. administrativer oder regulatorischer Institutionen, geeignet.

Der vorliegende Blutdruckkonsens 2019 steht in der Tradition der Empfehlungen der Österreichischen Gesellschaft für Hypertensiologie der letzten Jahrzehnte [1, 2]. In Anerkennung der Tatsache, dass Bluthochdruck eine echte Volkskrankheit darstellt und somit in der Allgemeinmedizin, aber auch in vielen Teilgebieten der Medizin eine wesentliche Rolle spielt, wurden die vorliegenden Empfehlungen gemeinsam von 12 medizinischen Fachgesellschaften sowie der Österreichischen Gesellschaft für Allgemeinmedizin erarbeitet. Das klare Ziel ist eine Verbesserung der Blutdrucksituation in Österreich.

\section{Blutdruck und kardiovaskuläres Risiko}

Unter 67 Risikofaktoren, die in der Global Burden of Disease Studie untersucht wurden, war systolischer Blutdruck (BD) $>115 \mathrm{~mm} \mathrm{Hg}$ der führende einzelne Risikofaktor für vorzeitige Sterblichkeit und für disability-adjusted life-years (DALYs) weltweit, verantwortlich für 9,4 Mio. Todesfälle und für 7,0\% der globalen DALYs 2010 [3]. Eine weitere Analyse dieser Studie fand, dass $14 \%$ aller weltweiten Todesfälle auf Hypertonie (systolischer BD >140 mm Hg) zurückgeführt werden können [4], wobei meist koronare Herzerkrankung und Schlaganfall Todesursachen waren. 53\% der DALYs durch koronare Herzerkrankung konnten auf erhöhten BD zurückgeführt werden [3]. Personen mit Hypertonie im Alter von 30 Jahren haben ein Risiko von 63,3\%, im weiteren Leben eine kardiovaskuläre Erkrankung zu erleiden, und sie erleiden diese 5 Jahre früher als Personen mit normalem Blutdruck [5]. In einer großen epidemiologischen Studie, an der 1,25 Mio. Personen (1/5 antihypertensiv behandelt) ohne kardiovaskuläre Erkrankung teilnahmen, zeigte sich, dass bis zum Alter von 79 Jahren ein systolischer $\mathrm{BD}<115 \mathrm{~mm} \mathrm{Hg}$ und ein diastolischer $\mathrm{BD}$ $<75 \mathrm{~mm}$ Hg mit dem niedrigsten Risiko für 12 unterschiedliche kardiovaskuläre Erkrankungen, inklusive stabile und instabile Angina pectoris, Myokardinfarkt, Herzinsuffizienz, plötzlicher Herztod, ischämischer und hämorrhagischer Schlaganfall sowie periphere arterielle Verschlusserkrankung assoziiert war [5]. Ein J-Kurven-Effekt, also ein höheres Risiko bei niedrigerem BD, bestand nicht. Eine frühere Meta-Analyse [6], die 1 Mio. Erwachsene aus 61 prospektiven Observationsstudien inkludierte, hatte ähnliche Ergebnisse: Während des gesamten Erwachsenenalters zeigte der BD einen starken und direkten Zusammenhang mit der kardiovaskulären Sterblichkeit, ohne Hinweis für einen unteren Schwellenwert, zumindest bis zu einem Wert von 115/75 mm Hg. Bei jungen Personen wies in der Framingham Studie der diastolische BD die stärkste Assoziation mit der koronaren Herzerkrankung auf [7], ab dem mittleren sowie in höherem Lebensalter (über 50-60 Lebensjahren) war ein höherer systolischer BD und ein niedrigerer diastolischer 
BD (d.h. ein höherer Pulsdruck) mit einem höheren Risiko für koronare Herzerkrankung verbunden [8].

Erhöhter BD verursacht in Frühstadien selten Symptome [9], und wird daher der „stille Killer“ genannt. Langfristig verursacht Bluthochdruck akzelerierte Atherosklerose (Plaques und Stenosen) und Arteriosklerose (erhöhte Gefäß-Steifigkeit), die beide zu Organschäden (z.B. an Herz, Gehirn, Nieren), klinischen Ereignissen, Behinderung und vorzeitigem Tod beitragen [9]. Weltweit ist etwa jeder 4. Mensch hypertensiv (definiert als $>140 / 90 \mathrm{~mm} \mathrm{Hg}$ ), sowie $30-45 \%$ aller Erwachsenen und mehr als $60 \%$ aller über 60 Jährigen. Weltweit ist die Anzahl an Personen mit systolischem BD >110-115 mm Hg (über diesem Wert steigt ja das kardiovaskuläre Risiko an) und mit Hypertonie (systolischer BD $>140 \mathrm{~mm} \mathrm{Hg}$ ) steigend [4]. Obwohl arterielle Hypertonie einen wichtigen genetischen Hintergrund hat, sind ihr Auftreten und ihre Behandlungsbedürftigkeit häufig verzögerbar. Eine frühe klinische Manifestation ist mit ungesundem Lebensstil (körperlicher Inaktivität, Überernährung, Übergewicht) assoziiert [10].

Trotz der durch große randomisierte Studien und deren Meta-Analysen nachgewiesenen Reduktion kardiovaskulärer Ereignisse durch antihypertensive Behandlung [11] sind Awareness, Behandlung und Kontrolle des Bluthochdrucks weiterhin ein großes Problem. In einer großen weltweiten Studie mit mehr als 140.000 Teilnehmern in Ländern mit hohem, mittlerem und niedrigem Einkommen [12], waren sich in den Ländern mit hohem Einkommen etwa 50\% der Hypertoniker ihrer Erkrankung bewusst. Beinahe $90 \%$ von ihnen waren behandelt, aber weniger als $50 \%$ der behandelten Hypertoniker hatten ihren BD gut kontrolliert. In den Ländern mit mittlerem und niedrigem Einkommen war die Situation schlechter.

\section{Fact Box Blutdruck und kardiovaskuläres Risiko}

- Erhöhter Blutdruck ist weltweit der wichtigste Risikofaktor für vorzeitige Sterblichkeit und Leben mit Behinderung.

- $14 \%$ aller weltweiten Todesfälle können auf Bluthochdruck zurückgeführt werden.

- In epidemiologischen Studien sind ein systolischer Blutdruck $<115 \mathrm{~mm} \mathrm{Hg}$ und ein diastolischer Blutdruck $<75 \mathrm{~mm} \mathrm{Hg}$ mit dem niedrigsten kardiovaskulären Risiko assoziiert.

- In höherem Alter ist die Kombination „erhöhter systolischer Blutdruck und erniedrigter diastolischer Blutdruck" besonders ungünstig, sie spricht für steife Gefäße.

\section{Die Situation in Österreich}

Gegenwärtig liegen folgende Erhebungen hinsichtlich der Kontrolle von Bluthochdruck in Österreich vor:

Die SCREEN-II-Studie (2003) [13] konnte zeigen, dass von 1303 Patientinnen und Patienten mit re- gelmäßigen Selbstmessungen (>30 /Patient/in, insgesamt 39.000 Messwerte), lediglich $17 \%$ das Heimblutdruckziel von 135/85 mm Hg unter Therapie erreichten. Ebenso waren nur $16 \%$ der Patient/innen, die ihren BD beim behandelnden Arzt/Ärztin messen ließen, adäquat behandelt. In der internationalen EURIKA-Studie (2010) [14] erreichten insgesamt 36\% von 624 in Österreich rekrutierten Patient/innen das Blutdruckziel von < 140/90 mm Hg.

Eine der rezentesten Erhebungen wurde im Oktober 2015 bei 158 teilnehmenden Apotheken in Niederösterreich durchgeführt. In einem zweiwöchigen Untersuchungszeitraum konnten 4303 Patient/innen eingeschlossen werden, welche aktiv eine Apotheke mit einem Rezept für ein Antihypertensivum aufsuchten. Die BD-Messung erfolgte standardisiert und entsprechend den Guidelines (an beiden Armen, nach 3-5 min Ruhephase, mit einem validierten automatischen oszillometrischen BD-Messgerät). In dieser Kohorte von diagnostizierten, behandelten und überwiegend therapietreuen Patient/innen erreichten nur $41 \%$ das Blutdruckziel von 140/90 mm Hg [15]. Dies ist ein alarmierender Wert, insbesondere da nichtadhärente Patient/innen kaum Berücksichtigung finden konnten und daher die Blutdruckkontrollrate in der behandelten Gesamtbevölkerung deutlich unter dem genannten Wert liegen dürfte. Der akzeptable Blutdruckdurchschnitt der Gesamtkohorte (144/84 mm Hg) täuscht darüber hinweg, dass Patient/innen mit kontrollierten Blutdruckwerten zwar adäquat eingestellt waren (126/77 mm Hg), jedoch die Mehrheit der nicht-kontrollierten Patient/innen das Therapieziel mit einem durchschnittlichem BD von 156/89 mm Hg deutlich verfehlte. Im Mittel erhielten die Patient/innen 1,8 antihypertensive Präparate, oder 2,2 Einzelsubstanzen, wenn man Kombinationspräparate in Betracht zog. Somit ist als mögliche Ursache für die unzufriedenstellende Blutdruckeinstellung die fehlende Aufmerksamkeit des Arztes/der Ärztin zur Therapieintensivierung („Physician's inertia“) in Betracht zu ziehen und nicht ein tatsächlich therapieresistenter Hypertonus [15]. Zukünftige Disease-Management Programme sollten sowohl patient/innenabhängige Faktoren (Therapietreue) als auch die Aufmerksamkeit der Ärzt/innen zur Therapieintensivierung als Hauptansatzpunkt in Betracht ziehen.

Ebenfalls in Apotheken wurden 2016-2017 in Oberösterreich bei 10.973 Personen BD und Gefäß-Steifigkeit (Pulswellengeschwindigkeit; ausgewertet als Gefäß-Alter) gemessen [16]. 38,1\% der Teilnehmenden dieser Untersuchung hatten einen erhöhten BD (>140/90 mm Hg). Von den 3890 Teilnehmenden mit bekannter Hypertonie waren 57,3\% in der Messung hypertensiv, von den 7083 Teilnehmenden ohne bekannte Hypertonie waren 29,5\% in der Messung hypertensiv. In einer Subgruppe wurde ein 24-h-Blutdruckmonitoring durchgeführt. Es zeigte sich, dass $80 \%$ der Personen mit erhöhtem BD in der Apothe- 
kenmessung auch im Alltag hypertensiv waren. Das Projekt bestätigt eine hohe Dunkelziffer bezüglich undiagnostizierter Hypertonie in Österreich, weiters die unzureichende Blutdruckkontrolle bei bekanntem Bluthochdruck.

Im Mai 2017 wurde im Rahmen der Aktion „May Measurement Month“ der International Society of Hypertension in 80 Ländern weltweit an unterschiedlichsten Plätzen BD-Screeningmessungen durchgeführt. In Österreich [17] beteiligten sich 56 Zentren, darunter niedergelassene Internist/innen und Allgemeinmediziner/innen, Spitalsambulanzen, Apotheken und Fitnessstudios an dieser Messaktion. Auch an zahlreichen öffentlichen Plätzen wurde Menschen über 18 Jahren der BD mittels automatisierter 3-fach Messung gemessen. Insgesamt wurden 2711 Personen gescreent, davon 58,6\% Frauen. 1704 (62,9\%) von diesen zeigten Blutdruckwerte $>140 / 90 \mathrm{~mm}$ Hg. Auch ein Datenbogen zur Evaluierung von Komorbiditäten und Medikamenteneinnahme wurde den Teilnehmenden ausgehändigt, wodurch sich zeigte, dass von den Probanden ohne antihypertensive Therapie 43,2\% hypertensiv waren - dies entspricht sozusagen den neu diagnostizierten Hypertoniker/innen. Besonders erschreckend war das Ergebnis bei den Personen, die bereits eine antihypertensive Therapie einnahmen: Hier zeigten 63,5\% hypertensive Werte und damit einen unkontrollierten Bluthochdruck. Die Ergebnisse dieser Messaktion unterstreichen die Wichtigkeit des Blutdruckscreenings bei Gesunden und die regelmäßige Therapiekontrolle bei therapierten Patientinnen und Patienten.

\section{Fact Box Blutdrucksituation in Österreich}

- In älteren Studien erreichten $17 \%$ (2003) bzw $36 \%$ (2010) der Patient/innen das Blutdruckziel.

- In einer neueren Studie (2015) mit Messung in Apotheken mit mehr als 4300 Patient/innen erreichten nur $41 \%$ der behandelten Hypertoniker/innen, die ein Rezept für ein Blutdruckmedikament einlösten, normotensive Blutdruckwerte.

- Eine weitere neue Studie (2016-2017) mit Apothekenmessungen an mehr als 10.000 Personen zeigte, dass $29,5 \%$ der Personen ohne bekannten Bluthochdruck und 57,3\% der Personen mit bekanntem (und meist behandeltem) Bluthochdruck hypertensive Blutdruckwerte aufwiesen.

- Im Rahmen der Aktion Messmonat Mai im Jahr 2017 wurden bei 2711 Personen 3-fach Blutdruckmessungen bei Ärzt/innen, in Apotheken und an öffentlichen Plätzen durchgeführt. 43,2 \% der unbehandelten Personen und $63,5 \%$ der behandelten Personen wiesen hypertensive Blutdruckwerte auf.

- Zusammenfassend sind die Raten an BlutdruckAwareness und Blutdruck-Kontrolle in Österreich nicht zufriedenstellend, und es besteht dringender Handlungsbedarf.

\section{Diagnose der Hypertonie - Blutdruckmessung}

\section{Einleitung}

Aufgrund der ausgeprägten Variation der absoluten Blutdruckhöhe (beat-to-beat; zirkadian; jahreszeitlich) [18] ist eine exakte Bestimmung der für ein Individuum typischen oder wahren Blutdruckhöhe nahezu unmöglich. Dementsprechend komplex gestaltet sich die Diagnose der arteriellen Hypertonie, wobei zur adäquaten Einschätzung der Blutdruckhöhe verschiedene immer komplexer werdende Messmethoden zur Verfügung stehen. Trotzdem sei an dieser Stelle darauf hingewiesen, dass sämtliche nationale und internationale Leitlinien für die Risikoeinschätzung und Behandlung der Hypertonie nicht nur den BD, sondern auch zusätzliche Parameter wie Risikofaktoren, Begleiterkrankungen, Endorganschäden und begleitende kardiovaskuläre Erkrankungen einfordern.

\section{Blutdruckmessmethoden}

Folgende Blutdruckmessmethoden stehen zur Beurteilung des BDs zu Verfügung:

a) Invasive beat-to beat Messung

b) Nicht-invasive beat-to-beat-Messung

c) Office-Blutdruckmessung konventionell

d) Office-Blutdruckmessung automatisiert

e) Office-Blutdruckmessung automatisiert ohne anwesendes medizinisches Personal („unattended“)

f) Blutdruckselbstmessung/Heimblutdruckmessung g) Ambulantes Blutdruckmonitoring

h) Blutdruckmessung im Rahmen der Ergometrie

Ad a Invasive Beat-to-beat Messung Obwohl diese Form der Blutdruckmessung den wissenschaftlichen Goldstandard darstellt, ist diese Meßform wegen ihrer Invasivität für die Diagnostik der Hypertonie ungeeignet. Sie wird vornehmlich in der Anästhesie/ Intensivmedizin und in der hypertensiologischen Forschung eingesetzt.

Ad b Nicht-invasive Beat-to beat Messung Diese Form der Blutdruckmessung erfolgt vor allem über eine oszillometrische Methode am Finger (Finapress, Task Force Monitor) [19]. Der Vorteil der Einzelschlagmessung wird allerdings im täglichen Gebrauch durch den Einfluss des hydrostatischen Drucks begrenzt. Gemeint ist damit die Veränderung des gemessenen BDs in Abhängigkeit von der Handhöhe im Vergleich zur Referenz Herzhöhe. Bei einem Niveau über Herzhöhe ist der BD fälschlich niedriger als real und vice versa. Diese Messmethode ist im Wesentlichen auf die Forschung sowie Spezialanwendungen wie z.B. die Kipptischuntersuchung beschränkt.

Ad c Office-Blutdruckmessung konventionell Die meisten großen epidemiologischen Studien, die den BD als unabhängigen Risikofaktor identifizierten, 


\section{Tab. 1 Richtiges Blutdruckmessen}

Vor der ersten Messung mindestens 5 min (z. B. Lesen einer Seite einer Zeitung) ruhiges Sitzen in einem Sessel mit den Beinen nicht-überkreuzt am Boden und dem Rücken angelehnt

Kein Kaffee, Sport, Rauchen 30 min vor der Messung

Kein Gespräch in der Wartephase und während der Messung

Anlage der Blutdruckmanschette direkt auf der Haut des Oberarmes (d. h. nicht über der Kleidung)

Verwendung eines validierten Messgeräts

Verwendung der richtigen Manschettengröße (Standardgrösse: 12-13cm Breite und $35 \mathrm{~cm}$ Länge; für dünnere bzw dickere Oberarme müssen kleinere bzw grössere Manschetten verwendet werden)

Der Arm mit der Blutdruckmanschette wird auf einem Tisch aufgestützt

Position der Mitte der Manschette in Herzhöhe (Mitte des Sternums)

Bei der ersten Messung wird an beiden Armen gemessen, in der Folge an dem Arm mit den höheren Messwerten

Wiederholung der Messung nach 1-2 min, dann nochmals, weitere Wiederholung falls die Messwerte um mehr als $10 \mathrm{~mm} \mathrm{Hg}$ differieren. Der Mittelwert der

letzten beiden Messungen wird notiert. Für die Selbstmessung ist es am besten, wenn das Blutdruckmessgerät die Werte automatisch speichert.

Falls auskultatorisch gemessen wird (allenfalls für die Praxismessung empfehlenswert): zunächst palpatorisch den systolischen Blutdruck abschätzen und die

Manschette dann 20-30 $\mathrm{mm} \mathrm{Hg}$ darüber aufblasen und langsam (2 $\mathrm{mm} \mathrm{Hg}$ pro Sekunde) ablassen.

Bei älteren Patienten, Diabetiker/innen und bei Orthostase-Symptomen wird eine Messung nach 1 und 3 min Stehen durchgeführt

Bei der Messung und bei der Palpation des Pulses wird auf Arrhythmien (Vorhofflimmern!) geachtet

Systolischen und diastolischen Blutdruck sowie Herzfrequenz notieren

Zeitlichen Zusammenhang zwischen der letzten Einnahme von Blutdrucksenkern und der Messung dokumentieren

Falls auskultatorisch gemessen wird, den systolischen und den diastolischen Blutdruck nicht auf-oder abrunden, sondern die nächste gerade Zahl notieren

Zur Bestimmung des Blutdruckniveaus zumindest 2 Einzelmessungen an zumindest 2 Messtagen mitteln

Die Messergebnisse dem Patienten/der Patientin mündlich und schriftlich mitteilen. Bei der Selbstmessung: Werte aufschreiben

Abb. 1 State-of-the-art der konventionellen OfficeBlutdruckmessung

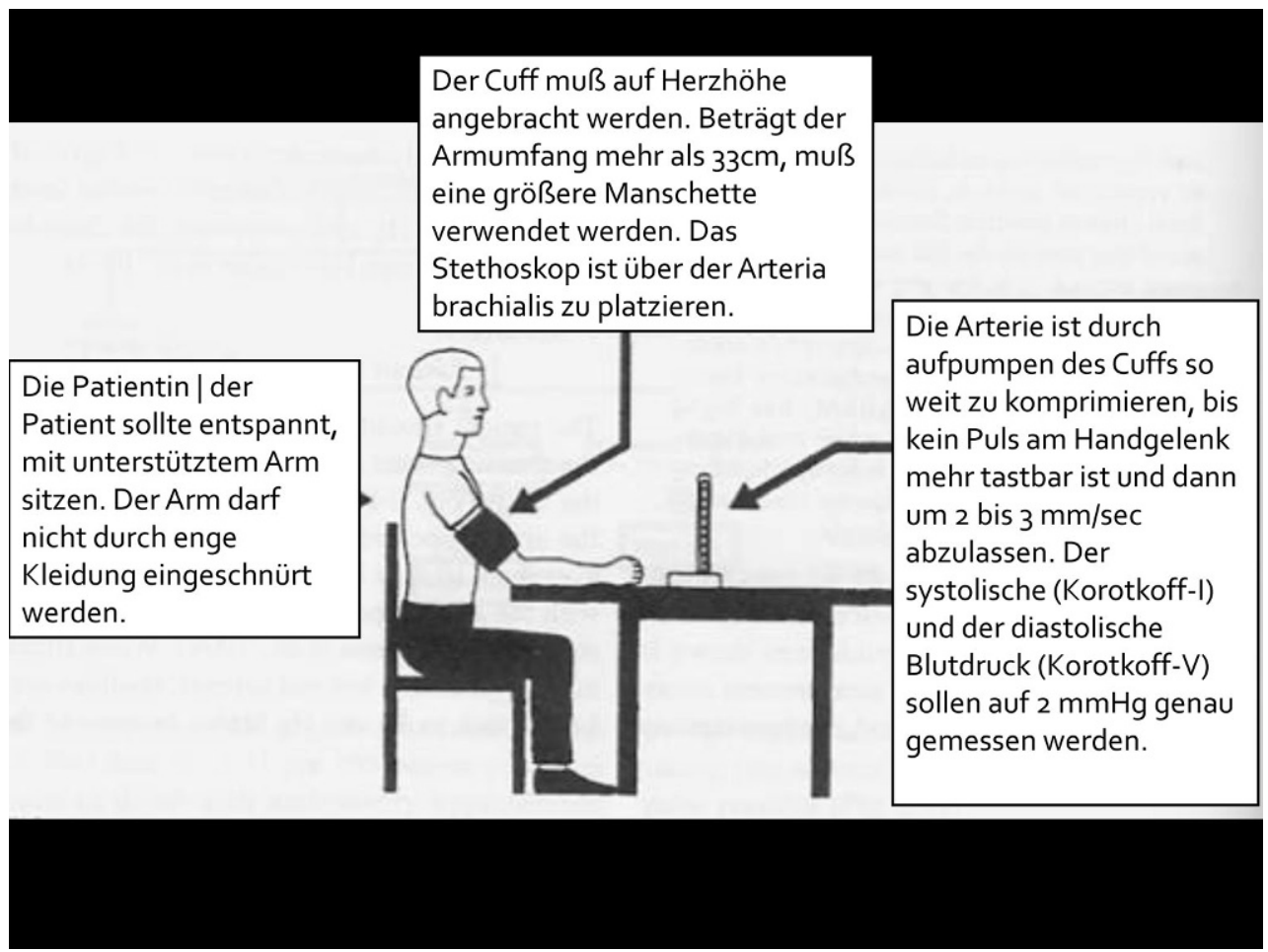

wurden auf Basis der konventionellen Office-Blutdruckmessung durchgeführt. Das gilt auch für nahezu alle Studien, die den vorteilhaften Effekt der medikamentösen Blutdrucksenkung auf Mortalität und Morbidität zeigen konnten. Dieses Messverfahren hat daher die stärkste Evidenzlage, ist aber für die Beurteilung des BDs von individuellen Patient/innen durch die Blutdruckvariabilität und durch in ihrem Ausmaß unvorhersehbare Weißkitteleffekte limitiert.

Entscheidend ist aufgrund der großen Blutdruckschwankungen auch bei Gesunden eine Standardisie- rung und damit hohe Qualität in der Durchführung dieser Messmethode (Tab. 1, Abb. 1).

Als erster Schritt ist die Verwendung eines validierten Blutdruckmessgerätes wichtig. Goldstandard bleibt die Methode nach Riva-Rocci und Korotkoff mit einem Quecksilber-Sphygmomanometer, wenngleich rezent durch eine große Meta-Analyse [20] belegt ist, daß die nichtinvasive Oberarm-Blutdruckmessung mit der Manschette im Vergleich $\mathrm{zu}$ invasiven Messungen den systolischen BD deutlich unterschätzt und den diastolischen $\mathrm{BD}$ deutlich über- 
schätzt. Alle anderen Blutdruckmessmethoden und -Geräte werden daran validiert. Der systolische BD ist dabei definiert als das Hörbarwerden des ersten Korotkoff-Geräusches, der diastolische BD als das Verschwinden des letzten Korotkoff-Geräusches. Aufgrund der Toxizität von Quecksilber sind diese Messgeräte nicht mehr breit verfügbar, und werden meist durch oszillometrische semi-automatische oder automatische Sphygmomanometer ersetzt. Bei der oszillometrischen Messung werden die Blutdruckschwankungen in der Blutdruckmanschette (die nur wenige $\mathrm{mm} \mathrm{Hg}$ umfassen) beim Ablassen derselben aufgezeichnet. Die Hüllkurve dieser geringen Blutdruckschwankungen ist im Idealfall diamantenförmig mit einem Maximum, das dem mittleren BD entspricht. Dieser wird daher oszillometrisch direkt und üblicherweise sehr präzise gemessen, aber meist gar nicht auf dem Display angezeigt. Aus der Hüllkurve der BD-Schwankungen in der Manschette wird mithilfe firmenspezifischer Algorithmen der systolische und der diastolische BD errechnet [21], wobei sich diese Algorithmen je nach Hersteller unterscheiden. Daher müssen alle automatischen oszillometrischen Blutdruckmessgeräte gegen den Gold-Standard geprüft („validert“) werden. Rezent konnte ein weltweit gültiger Standard zur Validierung der Messgeräte ausgearbeitet werden [22].

Zur Standardisierung sind eine ausreichende Ruhephase vor der ersten Messung und eine ruhige Messumgebung sowie eine entsprechende Körperhaltung mit Aufstützen des Messarmes erforderlich. Bei der ersten Messung sollte der BD an beiden Oberarmen gemessen werden, idealerweise simultan. Eine wiederholbare Blutdruckdifferenz zwischen beiden Armen von mehr als $15 \mathrm{~mm} \mathrm{Hg}$ systolisch ist ein Hinweis auf Atherosklerose und zeigt ein erhöhtes kardiovaskuläres Risiko an [23]. Die weiteren Blutdruckmessungen sollten am Arm mit dem höheren BD-Wert durchgeführt werden.

Die Bildung eines Mittelwerts aus mehreren Messungen, die in einem zeitlichen Abstand von 1-2 min durchgeführt werden, erhöht deren Aussagekraft in Bezug auf Diagnose, Prognose und Therapiekontrolle ganz erheblich. Der erste Messwert ist meist höher als die folgenden und sollte daher bei der Auswertung nicht berücksichtigt werden [24]. Daher empfehlen wir, den 1. Messwert von drei Messungen wegzulassen und lediglich den Mittelwert aus der Messung 2 und $3 \mathrm{zu}$ bilden. Falls die Messwerte um mehr als $10 \mathrm{~mm} \mathrm{Hg}$ differieren, sind weitere Messungen erforderlich. Dadurch wird vor allem die arousal-Reaktion durch den Messvorgang an sich vermindert. Die Diagnose einer Hypertonie kann im Allgemeinen erst nach mehreren BD-Messungen zu mehreren Gelegenheiten gestellt werden.

Besonders bei älteren Personen, Diabetiker/innen und bei entsprechenden Symptomen sollte eine Orthostasereaktion ausgeschlossen werden. Dazu sollte der BD auch nach 1 und 3 min Stehen gemessen wer- den. Orthostatische Hypotonie ist definiert als Abfall des systolischen/diastolischen BDs um 20/10 mm Hg innerhalb einer dreiminütigen Stehphase. Dies ist mit einem erhöhten kardiovaskulären Risiko verbunden [25] und wird häufig eine Therapieanpassung erforderlich machen.

Ad d Office-Blutdruckmessung automatisiert Es handelt sich hierbei um eine Verbesserung der konventionellen Office-Blutdruckmessung durch Verwendung automatisch arbeitender oszillometrisch messender Oberarm-Blutdruckmessgeräte, die auch selbständig Mittelwerte bilden können. Die größte Erfahrung mit dieser Form der Blutdruckmessung stammt aus Kanada [26]. Messfehler werden dadurch vermindert und die Aussagekraft der Office-Messung erhöht.

\section{Ad e Office-Blutdruckmessung automatisiert ohne} anwesendes medizinisches Personal („unbeobachtet") Bei dieser Messmethode befindet sich der Patient/die Patientin alleine in einem ruhigen Raum. Nach 5 min ruhigem Sitzen, Rücken angelehnt, ohne zu sprechen, wird der BD automatisch mit einem entsprechend programmierten oszillometrischen Oberarm-Messgerät gemessen. Verwendet wird der Mittelwert aus mindestens 3 Messungen im Abstand einer Minute. Diese Messmethode ist durch ihre Verwendung im Rahmen der SPRINT-Studie $\mathrm{zu}$ einer größeren Bekanntheit gelangt [27]. Die meisten Erfahrungen damit stammen ebenfalls aus Kanada. Der Hauptvorteil ist das weitgehende Vermeiden des Weißkitteleffektes. $\mathrm{Zu}$ Diskussionen Anlass gebend ist allerdings die Tatsache, dass solcherart erhobene Messwerte im Allgemeinen niedriger als Messungen mit konventionellen Office-Messungen liegen. Die mithilfe der automatischen Office-Messung erhobenen Blutdruckwerte dürften etwa um 10-15 mm Hg systolisch und um 5-7 mm Hg diastolisch tiefer als die konventionellen Office-Blutdruckwerte liegen [28]. In einem grossen Sample entsprach ein konventioneller Office-BD von 140/90 mm $\mathrm{Hg}$ einem automatischen Office-BD von 131/85 mm Hg [29]. Die Werte der automatischen Office-Messung liegen im Durchschnitt etwa auf Höhe der Tagesmittelwerte des 24-h-Blutdruckmonitoring und der Blutdruckselbstmessung [30], in der SPRINT Studie selbst aber etwa auf Höhe der 24-h-Durchschnittswerte [31]. Beim individuellen Patienten/bei der individuellen Patientin können aber beträchtliche Unterschiede bestehen [31], sodass die out-of-Office Messung durch die automatische Office-Messung nicht ersetzt wird. Ob die automatische Messung beobachtet oder unbeobachtet durchgeführt wird, dürfte sich auf die Blutdruckhöhe nicht wesentlich auswirken [32]. Jedenfalls liegen die Blutdruckgrenz- und -Zielwerte mit der automatischen Office-Blutdruckmessung deutlich tiefer als bei der konventionellen Office-Blutdruckmessung. 
Ad f Blutdruckselbstmessung/Heimblutdruckmessung Speziell in Österreich hat die Heimblutdruckmessung eine lange Tradition. Sie stellt eine outof-office-Messmethode dar, damit sind Weißkitteleffekte verhinderbar und longitudinale Blutdruckverläufe dokumentierbar. Schon aufgrund der höheren Anzahl der Messwerte und deren Erhebung im Alltag des Patienten ist die Selbstmessung der Office-Messung im Hinblick auf Korrelationen mit Endorganschäden [33] sowie in prognostischer Hinsicht [34] überlegen. Der Patient/die Patientin wird dadurch aktiver in das Management seiner/ihrer Erkrankung einbezogen, wodurch nachweislich die Blutdruckeinstellung durch bessere Compliance und Lebensstilveränderungen verbessert werden kann [35], besonders in Verbindung mit einem Schulungsprogramm [36]. Eine Titration der Antihypertensiva nach den Werten der Blutdruckselbstmessung resultiert in niedrigeren Blutdruckwerten im Vergleich zu einer OfficeBD basierten Therapie [37]. Dabei kann auch eine auf Blutdruckselbstmessung basierte Selbst-Titration nach einem festgelegten Schema hilfreich sein [38]. Das praktische Vorgehen ist in der Fact Box dargestellt. Eine wichtige Voraussetzung für die Blutdruckselbstmessung ist eine Schulung des Patienten/der Patientin hinsichtlich der Verwendung des Gerätes.

\section{Zusammenfassende Empfehlungen zur Verwendung der Heimblutdruckmessung}

Die Heimblutblutdruckmessung kann für 3 verschiedene Anwendungsbereiche herangezogen werden:

1. Diagnosesicherung der Hypertonie

2. Therapiekontrolle der Hypertonie

3. Unterstützung der Compliance + Adhärenz des Patienten/der Patientin im Sinne des Empowerments

Zur Diagnosesicherung/Ausschluß eines Weißkittelphänomens wird entsprechend den internationalen Guidelines eine „Blutdruckmesswoche“ empfohlen (Durchführung siehe Factbox Blutdruckselbstmessung). Ein Mittelwert $>135 / 85 \mathrm{~mm} \mathrm{Hg}$ bestätigt die Diagnose Hypertonie. Die Messwoche soll einmal pro Quartal durch den Patienten/die Patientin durchgeführt werden.

Zur Dokumentation einer ausreichenden Kontrolle der Hypertonie mittels Heimblutdruckmessung ist die publizierte Datenlage wenig belastbar. Aus pragmatischer Sicht bestehen 2 Möglichkeiten: Empfohlen wird die Durchführung einer „Blutdruckmesswoche“ pro Quartal (Durchführung siehe Factbox). Ein Mittelwert $<135 / 85 \mathrm{~mm}$ Hg zeigt eine ausreichende Therapiekontrolle an. Alternativ dazu kann aus statistischen Überlegungen die Verwendung des Blutdruck-Loads (= Prozentanzahl der Messungen $>135 / 85 \mathrm{~mm} \mathrm{Hg}$ ) erwogen werden. Wenn bei einmal täglichen Blutdruckmessungen $>25 \%$ der Messungen $>135 / 85 \mathrm{~mm}$ Hg liegen ( $>7$ Messungen pro 30 Tage), kann von einer un- zureichenden Hypertoniekontrolle ausgegangen werden.

\section{Fact Box Blutdruckselbstmessung}

- Verwendung eines geprüften automatischen Blutdruckmessgerätes

- Verwendung der richtigen Manschettengröße

- 5 min Ruhe vor den Messungen

- Sitzende Haltung, Oberarm aufgestützt

- Insgesamt etwa 30 Messungen

- Bei jeder Messung $3 \times$ messen und den Mittelwert der zweiten und dritten Messung bilden. Dieser Mittelwert wird gewertet.

- 1-2 min zwischen den Messungen warten

- 7 Tage morgens und abends messen

- Messungen schriftlich festhalten (oder bevorzugt am Gerät automatisch speichern)

- Ausgewertet wird der Mittelwert der Tage 2-7

Ad g Ambulantes Blutdruckmonitoring Diese Form der Blutdruckbeurteilung stellt den State-of-the-Art der nichtinvasiven Blutdruckmessung dar. Durch die Erhebung zahlreicher Messwerte (alle 15-20 min tagsüber, alle $30 \mathrm{~min}$ während der Schlafenszeit) ist ein repräsentativer 24-h-Querschnitt in Bezug auf die Blutdruckbelastung eines Individuums zu erheben. Als Qualitätskriterium gilt, dass zumindest $70 \%$ der Messwerte valide sind. Als Auswerteparameter stehen der mittlere BD über $24 \mathrm{~h}$, der Tagesmittelwert und der Nachtmittelwert zur Verfügung. Weiters stehen zusätzliche Parameter wie der zirkadiane Rhythmus des BDs (Dipping, Non-Dipping, Extreme-Dipping, Inverse-Dipping), der frühmorgendliche Blutdruckanstieg, und die Blutdruckvariabilität zur Verfügung [39].

Der 24-h-Mittelwert ist ein besserer Prädiktor für Hypertonie-assoziierte Endorganschäden [40] sowie kardiovaskuläre Ereignisse [41] als der Office-BD. Besondere prognostische Aussagen lassen sich aufgrund der Höhe des nächtlichen BDs (allerdings nur bei unbehandelten Patient/innen) treffen [42, 43]. Dieser ist normalerweise niedriger als der BD am Tag. Als arbiträrer Grenzwert dieses nächtlichen Blutdruckabfalls („Dipping“) wurde 10-20\% des Tagesblutdrucks gewählt, um normales Dipping zu charakterisieren. Typische Ursachen für geringeres nächtliches Dipping sind Schlafstörungen, obstruktive Schlafapnoe, Adipositas, orthostatische Hypotonie, autonome Dysfunktion, chronische Niereninsuffizienz, und höheres Alter [43]. Auch ein nächtlicher Blutdruckanstieg ist prognostisch ungünstig (,inverse Dipping“) [44].

\section{Fact Box Ambulantes Blutdruckmonitoring}

- Verwendung eines geprüften automatischen Blutdruckmessgerätes

- Verwendung der richtigen Manschettengröße 
- Indikation zur Diagnosesicherung und zur Therapiekontrolle

- Erfolgreiche Absolvierung des Kurses „Ambulantes Blutdruckmonitoring" der ÖGH

- Patient/inneninformation zgl Verhalten während des Messvorgangs + Tagebuch (Schlafzeiten, Aktivitäten ...)

- Normwerte $24 \mathrm{~h}<130 / 80 \mathrm{~mm} \mathrm{Hg}$ | Tag <135/ $85 \mathrm{~mm} \mathrm{Hg} \mid$ Nacht <120/70 mm Hg

- Beurteilung von Mittelwerten $24 \mathrm{~h} / \mathrm{Tag} / \mathrm{Nacht}$

- Beurteilung des zirkadianen Blutdruckprofils: Dipping | Non-Dipping | Extrem-Dipping | Inverses Dipping

Ad h Blutdruckmessung bei der Ergometrie Blutdruckanstiege bei Stresstests sind zum einen physiologisch, können aber andererseits zu diagnostischen und prognostischen Zwecken Verwendung finden. Physiologisch ist ein Anstieg des BDs um $10 \pm 2 \mathrm{~mm} \mathrm{Hg}$ pro MET (metabolisches Äquivalent), wobei der systolische BD stärker ansteigt als der diastolische. Der BD-Normalwert liegt bei maximaler Belastung systolisch <210 mm Hg (or) bzw <190 mm Hg (ㅇ) und diastolisch $<110 \mathrm{~mm} \mathrm{Hg}$. Der normale systolische Blutdruckanstieg (Ruhe versus maximale Belastung) liegt bei $50-60 \mathrm{~mm} \mathrm{Hg}$ ( $\left.\sigma^{7}\right)$ bzw. bei $40-50 \mathrm{~mm} \mathrm{Hg}$ (?). Allerdings ist auch eine Altersabhängigkeit zu berücksichtigen (bei höherem Alter sind stärkere Blutdruckanstiege normal). Für Aussagen zur kardiovaskulären Prognose ist vor allem der submaximale Blutdruck wertvoll (17) [45]. Ein überhöhter Blutdruckanstieg bei Belastung ist bei Personen mit noch normalem BD im Alltag mit einem erhöhten Risiko für das Auftreten einer Hypertonie assoziiert [46].

\section{Korrespondierende Blutdruckwerte mit Office- und out-of office Methoden}

Bei der Interpretation der Messwerte ist zu berücksichtigen, dass ambulante Blutdruckwerte nicht direkt mit Office-Messwerten vergleichbar sind. Bedingt u. a. durch das Weißkittelphänomen und ein Regression-to-the mean-Phänomen liegt der ambulante BD bei erhöhten Office-Werten tiefer (der Unterschied ist umso größer, je höher der Office-Blutdruckwert ist), aber bei niedrigem Office-BD mitunter auch höher als dieser. Somit sind die korrespondierenden Blutdruckwerte unterschiedlich, wobei sie sich bei niedri- gen Blutdruckwerten im Kollektiv annähern (Tab. 2). Bei Einzelpatient/innen kann die out-of-office Messung nicht durch Office-Messungen ersetzt werden.

\section{Weißkittelhochdruck („Praxishochdruck“, „White coat hypertension")}

Als Weißkittelhochdruck bezeichnet man bei unbehandelten Personen jene Situation, bei der der BD bei der Office-Messung erhöht, bei der out-of-office Messung (Selbstmessung, 24-h-Blutdruckmonitoring) aber normal ist. Der Blutdruckunterschied wird als „Weißkitteleffekt“ bezeichnet und ist eine Alarmreaktion auf Arzt/Ärztin oder in einem geringeren Ausmaß Pflegepersonal. Weißkittelhochdruck und Weißkitteleffekt (bei behandelten Patient/innen) ist häufig anzutreffen, besonders in höherem Alter und bei Frauen. Personen mit Weißkittelhochdruck haben im Vergleich zu normotensiven Individuen höhere Blutdruckwerte in der out-of office Messung (wenngleich noch im Normalbereich) und daher ein erhöhtes Risiko, später eine „echte“ Hypertonie zu bekommen. Sie haben mehr kardiovaskuläre Risikofaktoren, häufiger hypertensive Endorganschäden und auch eine schlechtere Langzeitprognose als echte Normotoniker [47]. Zur Absicherung ist sowohl Blutdruckselbstmessung als auch 24-h-Monitoring erforderlich, da nur bei Vorliegen eines normalen BDs in beiden Verfahren von einem niedrigen Risiko ausgegangen werden kann.

\section{Maskierte Hypertonie}

Als maskierte Hypertonie bezeichnet man bei unbehandelten Personen jene Situation, bei der der BD bei der Office-Messung normal, bei der out-of-office Messung (Selbstmessung, 24-h-Blutdruckmonitoring) aber erhöht ist. Bei behandelten Personen nennt man die entsprechende Situation „maskierte unkontrollierte Hypertonie“. Maskierte Hypertonie besteht häufiger bei schon grenzwertig hohem Office-BD. Maskierte Hypertonie findet man bei etwa $15 \%$ der Personen mit normalem Office-BD, häufiger bei jüngeren Personen, Männern, Raucher/innen, jenen mit höherem Ausmaß an körperlicher Aktivität, Stress im Beruf und Alkoholkonsum [18]. Häufig ist besonders der nächtliche BD erhöht. Maskierte Hypertonie ist mit mehr hypertensiven Endorganschäden sowie einer schlechteren Prognose als normaler BD assoziiert [41]. Das

Tab. 2 Korrespondierende Blutdruckwerte mit verschiedenen Messverfahren

\begin{tabular}{|c|c|c|c|c|}
\hline Office-Blutdruck & Blutdruck-Selbstmessung & $\begin{array}{l}\text { Tagesdurchschnitt } \\
\text { Blutdruckmonitoring }\end{array}$ & $\begin{array}{l}\text { Nachtdurchschnitt } \\
\text { Blutdruckmonitoring }\end{array}$ & $\begin{array}{l}\text { 24-h-Durchschnitt } \\
\text { Blutdruckmonitoring }\end{array}$ \\
\hline $120 / 80$ & $120 / 80$ & $120 / 80$ & $100 / 65$ & $115 / 75$ \\
\hline $130 / 80$ & $130 / 80$ & $130 / 80$ & $110 / 65$ & $125 / 75$ \\
\hline $140 / 90$ & $135 / 85$ & $135 / 85$ & $120 / 70$ & $130 / 80$ \\
\hline $160 / 100$ & $145 / 90$ & $145 / 90$ & $140 / 85$ & $145 / 90$ \\
\hline
\end{tabular}


Tab. 3 Einteilung des Blutdrucks nach OfficeMesswerten

\begin{tabular}{|l|c|c|c|}
\hline Blutdruck-Kategorie & Systolisch $\mathrm{mm} \mathrm{Hg}$ & \multicolumn{2}{c|}{ Diastolisch $\mathrm{mm} \mathrm{Hg}$} \\
\hline Optimal & $<120$ & und & $<80$ \\
\hline Normal & $120-129$ & und/oder & $80-84$ \\
\hline Hochnormal & $130-139$ & und/oder & $85-89$ \\
\hline Hypertonie Grad 1 & $140-159$ & und/oder & $90-99$ \\
\hline Hypertonie Grad 2 & $160-179$ & und/oder & $100-109$ \\
\hline Hypertonie Grad 3 & $\geq 180$ & und/oder & $\geq 110$ \\
\hline Isolierte systolische Hypertonie & $\geq 140$ & und & $<90$ \\
\hline
\end{tabular}

kardiovaskuläre Risiko liegt im Bereich der „echten Hypertonie“.

\section{Einsatz der verschiedenen Blutdruckmessverfahren}

Wie oben ausgeführt, bedingen Office-Messungen und out-of-office Messungen nicht selten die Zuordnung $\mathrm{zu}$ unterschiedlichen Blutdruckklassifizierungen. Bessere Übereinstimmungen gibt es zwischen ambulantem BD und den Heimblutdruckwerten, aber trotzdem sind diese Messverfahren nicht gegeneinander austauschbar, sondern sollten als einander ergänzende out-of-office-Blutdruckmessverfahren angesehen werden. Empfehlungen für den Alltag zeigt die Fact Box.

\section{Fact Box: Einsatz der unterschiedlichen Blut- druckmessverfahren}

- Diagnose der Hypertonie: Office-Blutdruck und Bestätigung durch Out-of-Office Messung (bevorzugt 24-h-Blutdruckmonitoring, bei Nicht-Verfügbarkeit Blutdruckselbstmessung)

- Therapiesteuerung bei medikamentöser Behandlung: Blutdruck-Selbstmessung ergänzt durch Office-Blutdruckmessung (bevorzugt automatisch)

- Bei Unklarheiten: 24-h-Blutdruckmonitoring als Goldstandard

\section{Diagnose und Einteilung der Hypertonie}

Aus epidemiologischer Sicht besteht ein kontinuierlicher Zusammenhang zwischen der Höhe des systolischen und des diastolischen BDs, beginnend $\mathrm{ab}$ systolischen Werten von unter $115 \mathrm{~mm} \mathrm{Hg}$ und diastolischen Werten von unter $75 \mathrm{~mm} \mathrm{Hg}[5,6]$, und dem Risiko für kardiovaskuläre Ereignisse. Ein eindeutiger Schwellenwert für die Diagnose „Hypertonie“ ist nicht fassbar. Aus praktischen Gründen, insbesonders zur Indikationsstellung für nichtmedikamentöse und medikamentöse Therapien zur Blutdrucksenkung, ist aber eine Klassifizierung erforderlich. Die Unterteilung, ob ein Bluthochdruck vorliegt oder nicht, richtet sich vorrangig nach der eindeutigsten Evidenzlage für einen Benefit einer medikamentösen Blutdrucksenkung, basierend auf dem Office-BD. Die Grenze normaler/erhöhter BD liegt dabei im Allgemeinen bei 140/90 mm Hg (Office-BD). Aus den oben angeführ- ten Limitationen folgt, dass 1. nicht-medikamentöse Maßnahmen zur Blutdrucksenkung schon bei niedrigeren Blutdruckwerten sinnvoll sein können, 2 . bei einzelnen Patient/innengruppen auch bei niedrigeren Blutdruckwerte eine Indikation zur medikamentösen Blutdrucksenkung bestehen kann, und 3. dass aufgrund der Variabilität des BDs out-of-Office Blutdruckmessungen erforderlich sind, um die Diagnose Hypertonie zu bestätigen.

Die empfohlene Klassifizierung, beruhend auf dem Office-BD, zeigt Tab. 3.

\section{Bluthochdruck und Begleiterkrankungen}

Hypertonie tritt selten isoliert auf, viel häufiger in Kombination mit anderen modifizierbaren (Rauchen, Übergewicht, Hyperlipidämie, Diabetes) und nichtmodifizierbaren (Alter, genetischer Hintergrund) kardiovaskulären Risikofaktoren. Dies gilt auch umgekehrt, so sind die meisten Patient/innen mit Typ2-Diabetes und/oder chronischer Niereninsuffizienz hypertensiv. Das globale kardiovaskuläre Risiko steigt mit der Anzahl an Risikofaktoren an [48, 49].

\section{Erweiterte Diagnose und Risikoeinschätzung}

Eine Möglichkeit zur Einschätzung des kardiovaskulären Risikos bietet der SCORE-Chart des European Society of Cardiology [49, 50]. Hier wird auf Basis von Alter, Geschlecht, systolischem BD, Raucherstatus und

Tab. 4 Erweitertes SCORE System zur kardiovaskulären Risikoeinschätzung

\begin{tabular}{|c|c|}
\hline \multirow{3}{*}{$\begin{array}{l}\text { Sehr hohes } \\
\text { Risiko }\end{array}$} & 10-Jahres-SCORE $\geq 10 \%$ \\
\hline & $\begin{array}{l}\text { Diabetes mellitus mit Organschaden und/oder schwere } \\
\text { Niereninsuffizienz (eGFR }<30 \mathrm{ml} / \mathrm{min} / 1,73 \mathrm{~m}^{2} \text { ) }\end{array}$ \\
\hline & $\begin{array}{l}\text { Klinisch manifeste oder durch Bildgebung eindeutig nachge- } \\
\text { wiesene kardiovaskuläre Erkrankung }\end{array}$ \\
\hline \multirow{3}{*}{$\begin{array}{l}\text { Hohes } \\
\text { Risiko }\end{array}$} & 10-Jahres-SCORE $\geq 5 \%<10 \%$ \\
\hline & $\begin{array}{l}\text { Diabetes mellitus ohne Organschaden oder relevanter singu- } \\
\text { lärer Risikofaktor (Hypertonie Grad 3, familiäre Hypercholes- } \\
\text { terinämie etc.) }\end{array}$ \\
\hline & LVH und/oder eGFR $30-59 \mathrm{ml} / \mathrm{min} / 1,73 \mathrm{~m}^{2}$ \\
\hline \multirow{2}{*}{$\begin{array}{l}\text { Moderates } \\
\text { Risiko }\end{array}$} & 10-Jahres-SCORE $\geq 1 \%<5 \%$ \\
\hline & Hypertonie Grad 2 \\
\hline $\begin{array}{l}\text { Niedriges } \\
\text { Risiko }\end{array}$ & 10-Jahres-SCORE $<1 \%$ \\
\hline
\end{tabular}




\section{konsensus bericht}

Tab. 5 Faktoren die das kardiovaskuläre Risiko bei Hypertonie beeinflussen

\begin{tabular}{|c|c|}
\hline \multicolumn{2}{|l|}{ Demographie und Laborparameter } \\
\hline Alter & - \\
\hline Männliches Geschlecht & - \\
\hline Rauchen & Rezent> Exraucher/in \\
\hline Hyperlipidämie & Gesamtcholesterin, LDL Cholesterin, HDL Cholesterin \\
\hline Hyperurikämie & - \\
\hline Diabetes mellitus & - \\
\hline Übergewicht und Adipositas & - \\
\hline $\begin{array}{l}\text { Positive Familienanamnese für kardiovaskulä- } \\
\text { re Erkrankungen }\end{array}$ & $M<55 J, F<65 J$ \\
\hline Früher Beginn der Menopause & - \\
\hline Bewegungsarmut & - \\
\hline $\begin{array}{l}\text { Psychosoziale und sozioökonomische Fakto- } \\
\text { ren }\end{array}$ & - \\
\hline Herzfrequenz & In Ruhe >80 Schläge/min \\
\hline \multicolumn{2}{|c|}{ Asymptomatische Hypertonie-vermittelte Organschäden } \\
\hline Erhöhte Gefäß-Steifigkeit & $\begin{array}{l}\text { Brachialis-Pulsdruck ab } 60 \mathrm{~mm} \mathrm{Hg} \\
\text { Carotis-femoralis oder aortale Pulswellengeschwindigkeit }>10 \mathrm{~m} / \mathrm{s}\end{array}$ \\
\hline Mäßiggradige asymptomatische Plaques [51] & $<50 \%$ Stenose in der Bildgebung (Angiographie, Ultraschall, CT, MRI) \\
\hline Linksherzhypertrophie & EKG- oder ECHO-Kriterien \\
\hline Mikroalbuminurie & $\begin{array}{l}30-300 \mathrm{mg} / 24 \text { Std oder Albumin-Kreatinin-Ratio }>300 \mathrm{mg} / \mathrm{g} \text { (>34 mg/ } \\
\mathrm{mmol} \text { ) }\end{array}$ \\
\hline Mäßiggradige Niereninsuffizienz & eGFR $30-59 \mathrm{ml} / \mathrm{min} / 1,73 \mathrm{~m}^{2}$ \\
\hline Knöchel-Arm Index pathologisch & $<0,9$ oder $>1,4$ \\
\hline Fortgeschrittene Retinopathie & Blutungen, Exsudate, Papillenödem \\
\hline \multicolumn{2}{|l|}{ Manifeste kardiovaskuläre oder renale Erkrankung } \\
\hline Zerebrovaskuläre Erkrankung & Ischämischer oder hämorrhagischer Insult, TIA \\
\hline Koronare Herzerkrankung & Myokardinfarkt, Angina, Revaskularisation \\
\hline Höhergradige asymptomatische Plaques & $>50 \%$ Stenose in der Bildgebung (Angiographie, Ultraschall, CT, MRI) \\
\hline Herzinsuffizienz & Unabhängig von der $\mathrm{EF}$ \\
\hline Periphere arterielle Verschlusserkrankung & Inklusive Aortenaneurysma \\
\hline Vorhofflimmern & - \\
\hline Höhergradige Niereninsuffizienz & $\mathrm{eGFR}<30 \mathrm{ml} / \mathrm{min} / 1,73 \mathrm{~m}^{2}$ \\
\hline
\end{tabular}

Abb. 2 Hypertonie-Stadien und kardiovaskuläres Risiko (niedrig-mäßig-hoch-sehr hoch), exemplarisch dargestellt für einen Mann im mittleren Alter (RF Risikofaktoren, HVOS Hypertonievermittelte Organschäden, NI 3 Niereninsuffizienz 3 (eGFR $30-59 \mathrm{ml} / \mathrm{min} / 1,73 \mathrm{~m}^{2}$ ), NI 4 Niereninsuffizienz 4 (eGFR $<30 \mathrm{ml} / \mathrm{min} / 1,73 \mathrm{~m}^{2}$ ), $\mathrm{Dm}$ Diabetes mellitus, CV$\mathrm{KH}$ manifeste kardiovaskuläre Erkrankungen). Adaptiert nach Williams et al. [52]

\begin{tabular}{|c|c|c|c|c|c|}
\hline Stadium der & $R F, O S$ & BD & Hypertonie & Hypertonie & Hypertonie \\
\hline Hypertonie & $C V-K H$ & hochnormal & Grad 1 & Grad 2 & Grad 3 \\
\hline \multirow{4}{*}{$\begin{array}{l}\text { Stadium } 1 \\
\text { (unkompliziert) }\end{array}$} & & $130-139 / 80-89$ & $140-159 / 90-99$ & $\begin{array}{c}160-179 / 100- \\
109\end{array}$ & $\geq 180 / \geq 110$ \\
\hline & $0 \mathrm{RF}$ & Niedrig & Niedrig & Mässig & Hoch \\
\hline & $1-2 \mathrm{RF}$ & Niedrig & Mässig & Mässig-hoch & Hoch \\
\hline & $\geq 3 \mathrm{RF}$ & $\begin{array}{l}\text { Niedrig- } \\
\text { mässig }\end{array}$ & Mässig-hoch & Hoch & Hoch \\
\hline $\begin{array}{l}\text { Stadium } 2 \\
\text { (asymptomatische }\end{array}$ & $\begin{array}{l}\text { HVOS, } \\
\text { Dm, }\end{array}$ & Mässig-hoch & Hoch & Hoch & $\begin{array}{c}\text { Hoch bis sehr } \\
\text { hoch }\end{array}$ \\
\hline Erkrankung) & NI 3 & & & & \\
\hline Stadium 3 & $\mathrm{Dm}$ & Sehr hoch & Sehr hoch & Sehr hoch & Sehr hoch \\
\hline (symptomatische & OS, & & & & \\
\hline \multirow[t]{2}{*}{ Erkrankung) } & NI 4, & & & & \\
\hline & CV-KH & & & & \\
\hline
\end{tabular}


Gesamtcholesterinspiegel das absolute 10-Jahres-Risiko für kardiovaskulären Tod angegeben, wobei für Österreich der sogenannte „low risk“-Chart anzuwenden ist. Für jüngere Personen, die alleine aufgrund des Alters ein niedriges absolutes Risiko aufweisen, wird auch das relative Risiko angegeben. Als sehr hoch bezeichnet man ein mittels SCORE kalkuliertes 10-Jahres-Risiko von $>10 \%$, als hoch eines von $5-10 \%$, als mäßig eines von $1-5 \%$ und als gering eines von $<1 \%$.

In Ergänzung zum SCORE-Chart liegt ein sehr hohes kardiovaskuläres Risiko vor, wenn schon kardiovaskuläre Erkrankungen (inklusive höhergradige asymptomatische Stenosen) vorliegen, oder ein Typ2-Diabetes mit Organschäden, oder eine fortgeschrittene Niereninsuffizienz (Tab. 4).

In Ergänzung zum SCORE-Chart liegt ein hohes kardiovaskuläres Risiko vor, wenn hypertensive Organschäden feststellbar sind, wenn ein Typ-2Diabetes besteht, oder eine mäßiggradige Niereninsuffizienz, oder wenn ein singulärer Risikofaktor außerordentlich erhöht ist (z.B. Hypertonie Grad 3, Gesamtcholesterin $>310 \mathrm{mg} / \mathrm{dl}$, etc).

In Ergänzung zum SCORE-Chart liegt ein mäßiggradiges kardiovaskuläres Risiko vor, wenn der (Office)BD > 160/100 mm Hg liegt.

Einen Überblick über weitere Faktoren, die das kardiovaskuläre Risiko determinieren, sowie eine genauere Spezifizierung von Organschäden und kardiovaskulären/renalen Erkrankungen zeigt Tab. 5.

Abhängig vom Vorliegen von Organschäden und manifesten Herz-Kreislauferkrankungen wird die Hypertonie in verschiedene Stadien eingeteilt, die das kardiovaskuläre Risiko widerspiegeln (Abb. 2; [52]).

\section{Basisabklärung bei Hypertonie: Anamnese, Status, Basislabor}

\section{Einleitung}

Ziel der klinischen Evaluation ist es, potenzielle Ursachen zu identifizieren, welche zur Entstehung des Hypertonus beitragen (Lebensstilfaktoren, Begleitmedikation, familiäre Belastung) und weitere kardiovaskuläre Risikofaktoren, atherosklerotische HerzKreislauferkrankungen und Hypertonie-vermittelte Organschäden (HVOS) zu erheben. Daraus leitet sich insofern eine direkte therapeutische Konsequenz ab, als dass die Substanzwahl (s. Abschn. 11) und das Blutdruckziel (s. Abschn. 12) entscheidend von diesen Faktoren mitbestimmt sind [52, 53].

\section{Anamnese}

Bei Erstdiagnose einer arteriellen Hypertonie und auch bei fehlendem Therapieerfolg im Sinne einer therapieresistenten Hypertonie ist eine zielgerichtete Anamnese unerläßlich, um die weitere therapeutische Strategie optimal gestalten zu können.
Nachfolgend sind wesentliche Aspekte der Anamnese in tabellarischer Form zusammengefasst. Die ausführliche Darstellung soll bei der Anamneseerhebung hinsichtlich eines möglichen HVOS und bei Verdacht auf sekundäre Hypertonieursachen als Hilfestellung zur gezielten klinischen Evaluation dienen (Tab. 6).

\section{Status}

Der klinisch physikalische Status erlaubt Rückschlüsse auf Komorbiditäten, hypertensive Endorganschäden und sekundäre Hypertonieursachen zu ziehen. Methoden zur korrekten Blutdruckmessung sind in Abschn. 3 zusammengefasst. Weitere routinemäßig empfohlene klinische Untersuchungen beinhalten (Tab. 7).

\section{Basislabor}

Das Basislabor dient einerseits zur Identifikation möglicher kardiovaskulärer Komorbiditäten und ist darüber hinaus essentiell, um eine sichere und effektive medikamentöse Therapie etablieren bzw. intensivieren zu können. Folgende Laborbestimmungen sind zur Basisabklärung empfohlen (Tab. 8).

Zusätzlich wird ein 12-Ableitungs-EKG empfohlen.

\section{Hypertonie-vermittelte Organschäden (struk- turelle und funktionelle Veränderungen durch er- höhten Blutdruck)}

\section{Einleitung}

Die Diagnostik von asymptomatischen, funktionellen und strukturellen Veränderungen der Blutgefässe (Arterien, Arteriolen und Mikrozirkulation) sowie der Endorgane (Herz, Gehirn, Augen, Nieren) durch erhöhten BD (Hypertonie-vermittelte Organschäden - HVOS) ist pathophysiologisch [54] und diagnostisch [55] wichtig und prognostisch relevant. In einer Populationsstudie aus Dänemark [56] war das 10Jahres-Risiko für kardiovaskulären Tod bei SCORE unter 5\% und Fehlen von HVOS niedrig. Das isolierte Vorliegen von HVOS bzw. SCORE $\geq 5 \%$ erhöhte das Risiko 4-fach bzw 5,9-fach, die Kombination beider 17-fach. Je mehr HVOS vorlagen (Linksherzhypertrophie, erhöhte Gefäß-Steifigkeit, Carotisplaques, erhöhte Albuminausscheidung im Harn), desto höher war das Risiko. HVOS spielen in der Stadieneinteilung der Hypertonie eine wichtige Rolle und haben auch unmittelbare therapeutische Implikationen. Nur bei fehlendem Nachweis von Organschäden kann bei unbehandelter Grad 1 Hypertonie mit niedrigmoderatem kardiovaskulären Risiko ein konservatives Procedere mit „Lifestyle-Intervention“ ohne medikamentöse Therapie für 3-6 Monate empfohlen werden [1, 52]. Auf der anderen Seite stellt das Vorliegen von HVOS bei Grad 1 Hypertonie eine klare Indikation 
Tab. 6 Anamnese bei Bluthochdruck. (Modifziert nach Williams et al. [52])

\begin{tabular}{l} 
Allgemeine Anamnese \\
Beschwerden, Symptome \\
Erstdiagnose der arteriellen Hypertonie \\
Frühere und aktuelle Blutdruckwerte \\
Frühere und aktuelle antihypertensive Therapie inkl. Nebenwirkungen und \\
Therapieadhärenz \\
Begleitmedikation \\
Risikofaktoren \\
\hline Lebensstilfaktoren \\
Familienanamnese, Ernährungsgewohnheiten inkl. Alkoholkonsum und \\
Salzkonsum, Nikotin- und Drogenabusus, sportliche Aktivität, Schlafana- \\
mnese, ggf. Einfluss der Therapie auf die Sexualfunktion \\
\hline Kardiovaskuläre Risikofaktoren \\
Diabetes mellitus, Hyperlipidämie, Familienanamnese \\
Begleiterkrankungen, stattgehabte kardiovaskuläre Ereignisse \\
\hline $\begin{array}{l}\text { Myokardinfarkt, Schlaganfall, Niereninsuffizienz, CAVK, PAVK, erektile } \\
\text { Dysfunktion, Partneranamnese hinsichtlich Schlafapnoe/Schnarchen, Hy- } \\
\text { pertonie in der Schwangerschaft/Eklampsie }\end{array}$ \\
\hline
\end{tabular}

Symptome welche auf einen Hypertonie-assoziierten Organschaden hindeuten können

Zerebral

Kopfschmerz, Schwindel, Synkope, Sehstörungen, sensorisches oder motorisches Defizit, kognitive Störungen

Kardial

Angina pectoris, Kurzatmigkeit, Ödeme, Synkope, Palpitationen

Renal

Durst, Polyurie, Polydipsie, Hämaturie, Familienanamnese hinsichtlich chronischer Niereninsuffizienz oder polycystischer Nierenerkrankung

Peripher arteriell

Kalte Extremitäten, Claudicatio intermittens, schmerzfreie Gehstrecke

Anamnese hinsichtlich potenziell sekundärer Ursachen

Früher Beginn einer Hypertonie 2. oder 3. Grades (<40 Jahren)

Plötzliches Eintreten oder rasche Verschlechterung eines arteriellen Hypertonus, repetitive Schweißausbrüche, Panikattacken, Kopfschmerzen, Palpitationen (Phäochromozytom)

Begleittherapie mit NSAR, Kortikosteroiden, nasalen Vasokonstriktoren, Chemotherapeutika (insb. Paclitaxel, Cisplatin, VEGF Inhibitoren, div. monoklonale Antikörper, Tyrosinkinaseinhibitoren), Immunsuppresiva (Cyclosporin, Tacrolimus), Serotonin-Noradrenalin-Wiederaufnahmehemmer (Venlafaxin, Milnacipran, Duloxetin), Erythropoetin, insb. östrogenhaltige orale Kontrazeptiva

Drogenabusus, Energy Drinks, alternativmedizinische Präparate, Lakritze, Anabolika

Spontane- oder diuretikainduzierte Hypokaliämien, Muskelschwäche, Tetanie (Hyperaldosteronismus - Conn Syndrom)

Symptome von Schilddrüsenerkrankungen/Hyperthyreose/

Hyperparathyreodismus

Schlafanamnese (Partneranamnese hinsichtlich Apnoe und Schnarchen)

Bekannte Erkrankung der Nieren oder des Harntraktes

Schwangerschaft

für eine medikamentöse Behandlung dar. Selbst bei hoch-normalen Blutdruckwerten und dem Vorliegen von HVOS kann eine medikamentöse Therapie in Betracht gezogen werden.

Die wichtigste Rolle spielt das Screening auf HVOS bei Personen mit niedrigem oder intermediärem kar-
Tab. 7 Physikalische Untersuchung bei Bluthochdruck. (Modifziert nach Williams et al. [52])

\begin{tabular}{|l|l|}
\hline Physiognomie & $\begin{array}{l}\text { Körpergewicht, Größe, Ernährungszustand, Bauch- } \\
\text { umfang }\end{array}$ \\
\hline $\begin{array}{l}\text { Hypertonie-assoziier- } \\
\text { ter Organschaden }\end{array}$ & $\begin{array}{l}\text { Neurostatus, kognitiver Status, Fundoskopie, } \\
\text { Auskultation des Herzens, der Karotiden und der } \\
\text { Nierenarterien, Pulsstatus, Blutdruckseitendiffe- } \\
\text { renz }\end{array}$ \\
\hline $\begin{array}{l}\text { Sekundäre Hyperto- } \\
\text { nie }\end{array}$ & $\begin{array}{l}\text { Auskultation Herz, Nierenarterien (Vitien, renovas- } \\
\text { kuläre Hypertonie) } \\
\text { Zeichen eines M. Cushing/Cushing Syndrom } \\
\text { (Stammfettsucht, ,Stiernacken“, Striae rubra, } \\
\text { Muskelatrophie, Akne, Hirsutismus, Akromegalie } \\
\text { etc.) } \\
\text { Schilddrüsenpalpation } \\
\text { Palpation Nierenlager (Polycystische Nierenerkran- } \\
\text { kung) } \\
\text { Cafe-au-lait Flecken (Neurofibromatose - Phäo- } \\
\text { chromozytom) }\end{array}$ \\
\hline
\end{tabular}

Tab. 8 Basislabor bei Bluthochdruck. (Modifiziert nach Williams et al. [52])

\begin{tabular}{l} 
Basislabor bei Hypertonie \\
Blutbild \\
\hline Nüchternblutzucker, HbA1c \\
Serumlipide: Gesamtcholesterin, LDL Cholesterin, HDL Cholesterin, Triglyce- \\
ride \\
\hline Serumkreatinin und berechnete GFR \\
\hline Serumelektrolyte: Natrium, Kalium \\
\hline Harnsäure \\
\hline Leberfunktionsparameter: GOT (ASAT), GPT (ALAT), Gamma-GT, alkalische \\
Phosphatase, Cholinesterase, Lipase, Bilirubin \\
\hline Urinstreifen: Protein, Glucose (falls verfügbar Albumin/Kreatinin Ratio) \\
\hline
\end{tabular}

diovaskulärem Risiko, basierend auf den klassischen Risikofaktoren (SCORE-System). Hier bewirkt das Vorliegen von HVOS eine Reklassifizierung (niedriges $\rightarrow$ intermediäres bzw. intermediäres $\rightarrow$ hohes kardiovaskuläres Risiko).

Auch Veränderungen der Organschäden im Verlauf sind zu erheben [57]. Eine Abnahme der linksventrikulären Hypertrophie (LVH) unter medikamentöser Therapie geht mit einer verbesserten Prognose einher [58]. In einer Meta-Analyse wiesen Patient/innen mit persistierend normaler linksventrikulärer Masse bzw. Regression einer LVH ein halb so hohes kardiovaskuläres Risiko auf wie Patient/innen mit persistierender LVH oder neuem Auftreten einer LVH. Ähnliches gilt auch für eine Abnahme der Albuminurie [59] unter antihypertensiver Medikation, während eine Reduktion der Intima-Media Dicke in der Arteria carotis mit keiner prognostischen Verbesserung assoziiert ist.

\section{Fact Box: asymptomatische Hypertonie-vermit- telte Organschäden}

\section{Arterielles Gefäß-System}

- Knöchel-Arm-Index $<0,9$

- Pulsdruck (bei älteren Menschen) $\geq 60 \mathrm{~mm} \mathrm{Hg}$ 
- Carotis-femoralis (aortale) Pulswellengeschwindigkeit $(>10 \mathrm{~m} / \mathrm{s})$

\section{Herz: Linksventrikuläre Hypertrophie}

- EKG

- Sokolow-Lyon Index $\left(\mathrm{S}_{\mathrm{V} 1}+\mathrm{R}_{\mathrm{V} 5}\right)>35 \mathrm{~mm}$

- $R_{\mathrm{aVL}} \geq 11 \mathrm{~mm}$

- Cornell Voltage $S_{V 3}+R_{a V L}>28 m m$ bei Männern bzw. $>20 \mathrm{~mm}$ bei Frauen

- Cornell Voltage $\times$ QRS Dauer >2440 mm.ms

- Echokardiographie

- LV Massenindex/Körpergröße $e^{2,7}$ : >50 g/m Männern bzw. $>47 \mathrm{~g} / \mathrm{m}^{2,7}$ bei Frauen

- LV Massenindex /BSA: $>115 \mathrm{~g} / \mathrm{m}^{2}$ bei Männern bzw. $>95 \mathrm{~g} / \mathrm{m}^{2}$ bei Frauen

\section{Niere}

- Albuminausscheidung

- Mikroalbuminurie (30-300 mg in 24 h)

- Albuminurie (>300 mg in $24 \mathrm{~h}$ )

- erhöhte Harn Albumin-Kreatinin-Ratio (30-300 mg/g; 3,4-34 mg/mmol) (bevorzugt im Morgenharn gemessen)

- Nierenfunktionsstörung

- Stadium III: eGFR 30-59 mL/min/1,73 m²

- Stadium IV: eGFR $15-30 \mathrm{~mL} / \mathrm{min} / 1,73 \mathrm{~m}^{2}$

- Stadium V: eGFR $<15 \mathrm{~mL} / \mathrm{min} / 1,73 \mathrm{~m}^{2}$

\section{Augenhintergrund}

- hämorrhagisches oder exsudatives Papillenödem

\section{Diagnostik von Hypertonie-vermittelten Organschäden}

Als basale Screeningtests werden die Ableitung eines EKG, die Messung der Albumin-Kreatinin-Ratio im Harn und die Messung des Serum-Kreatinins bzw. die Abschätzung der eGFR bei allen Patient/innen mit Bluthochdruck empfohlen.

Als weiterführende Untersuchungen bei asymptomatischen Hypertoniker/innen werden die Echokardiographie, die Sonographie der Gehirn-versorgenden Gefäße (Carotis-Vertebralis-Duplex), der abdominelle Ultraschall (Niere, Aorta, Nebenniere, Nierenarterien), Pulswellengeschwindigkeit und Knöchel-Arm-Index empfohlen, wenn das Ergebnis der Untersuchung(en) eine Änderung der Therapie bewirken könnte, z.B. durch Reklassifizierung in eine höhere Risikokategorie. Kongnitive Tests und eine zerebrale Bildgebung (CT/MRI) sind meist nur bei Symptomen indiziert. Die Durchführung einer Fundoskopie ist besonders bei Blutdruckwerten $>160 / 100 \mathrm{~mm} \mathrm{Hg}$ (Office-BD) sinnvoll.

\section{Das Herz bei arterieller Hypertonie}

Folgeschäden eines Bluthochdrucks sind die Linksventrikelhypertrophie (LVH), eine gestörte linksventrikuläre Relaxation („steifes Herz“), eine Vergrößerung des linken Vorhofs mit gehäuften Arrhythmien (vor allem Vorhofflimmern) und ein erhöhtes Risiko für das Entstehen einer Herzinsuffizienz (sowohl mit reduzierter EF - HFrEF als auch mit erhaltener EF HFpEF).

Bei allen Patient/innen mit arterieller Hypertonie sollte ein 12-Ableitungs-EKG dokumentiert werden, wenngleich dieses für die LVH nur eingeschränkt sensitiv ist. Allerdings haben EKG Veränderungen (Hypertrophiekriterien; „strain pattern“ - also Repolarisationsstörungen) eine gut etablierte prognostische Relevanz.

Die transthorakale 2D-Echokardiographie ist ein wesentlicher Bestandteil in der Abklärung der arteriellen Hypertonie [60]. Die schnelle Verfügbarkeit bei fehlender Belastung durch ionisierende Strahlung rechtfertigt den breiten klinischen Einsatz. Im Rahmen der Abklärung bei Hypertonie sind LVH, LV Geometrie und Grösse des linken Vorhofs von besonderer prognostischer Relevanz. Jedenfalls indiziert ist eine Echokardiographie bei allen Patient/innen mit pathologischem EKG und/oder Zeichen einer linksventrikulären Dysfunktion, weiters bei Symptomen wie Dyspnoe. Bei Nachweis einer LVH sollte auch bei Vorhandensein einer arteriellen Hypertonie an häufige Begleiterkrankungen gedacht werden, die ebenso zu einer Verdickung der linkventrikulären Wand und des Septums führen (z.B. Aortenstenose bzw. senile kardiale Amyloidose bei älteren Patient/innen, hypertrophe Kardiomyopathie bei jüngeren Patient/ innen). Weiterführende Untersuchungen (3D-Echokardiographie, Herz-MRI) sind derzeit weitgehend wissenschaftlichen Fragestellungen vorbehalten. Für die Beurteilung der diastolischen Funktion der linken Herzkammer [60, 61] werden Gewebsdoppler, Einstromgeschwindigkeit an der Mitralklappe, Größe des linken Vorhofs und Rückstromgeschwindigkeit an der Trikuspidalklappe herangezogen (Tab. 9).

\section{Die Blutgefäße bei arterieller Hypertonie}

\section{Arteria Carotis}

Das Vorliegen von Plaques im Carotis-Ultraschall wird meist als eine Verbreiterung der Intima-media Dicke (IMT $>1,5 \mathrm{~mm}$, oder fokale Verdickung um $>0,5 \mathrm{~mm}$ oder um $>50 \%$ zur unmittelbaren Umgebung) definiert und hat prognostische Bedeutung zusätzlich zu den klassischen Risikofaktoren [56, 62]. Die IMT selbst (IMT $>0,9 \mathrm{~mm}$ in der Arteria carotis wird als pathologisch angesehen) hat geringere prognostische Bedeutung.

Hochgradige Plaques ( $\geq 50 \%$ ) gelten als unabhängiger Risikomarker für Insult bzw. Herzinfarkt und füh- 
Tab. 9 Echokardiographie-Kriterien für kardiale Endorganschäden

\begin{tabular}{l|c|}
\hline $\begin{array}{l}\text { Echoparameter } \\
\text { LVH - LVMI/BSA }\end{array}$ & Grenzwerte \\
\hline LVH - LVMI/Körpergröße 2,7 & Fänner $>115 \mathrm{~g} / \mathrm{m}^{2}$ \\
& Männer $>50 \mathrm{~g} / \mathrm{m}^{2,7}$ \\
\hline LV Geometrie - RWT & Frauen $>47 \mathrm{~g} / \mathrm{m}^{2,7}$ \\
\hline LV Dilatation (LVEDD/Körpergröße) & $>0,42$ \\
\hline Linker Vorhof (Volumen/Körperoberfläche) & Männer $>34 \mathrm{~mm} / \mathrm{m}$ \\
\hline Linksventrikuläre Wanddicke (Septum, posterior) & Frauen $>33 \mathrm{~mm} / \mathrm{m}$ \\
\hline $\begin{array}{l}\text { BSA body surface area, LVEDD left ventricular enddiastolic diameter, LVHleft ventricular hypertrophy, LVMIleft ventricular } \\
\text { mass index, RWT relative wall thickness }\end{array}$
\end{tabular}

ren automatischer zur Zuordnung in die Kategorie „sehr hohes Risiko“ [52].

\section{Gefäßsteifigkeit}

Eine zunehmende Steifigkeit der großen Gefäße ist der Hauptgrund für die isolierte systolische Hypertonie im Alter. Dies führt zu einem erhöhten Pulsdruck (bei älteren Personen pathologisch wenn $\geq 60 \mathrm{~mm} \mathrm{Hg}$ ). Als diagnostischer Goldstandard der Gefäßsteifigkeit gilt die Messung der Pulswellengeschwindigkeit (PWV; Grenzwert $>10 \mathrm{~m} / \mathrm{s}$ ) zwischen Arteria carotis und Arteria femoralis [63]. Die PWV hat prognostische Bedeutung über die klassischen Risikofaktoren hinaus [64]. Eine Verbesserung der PWV ist vorteilhaft, dies wurde zumindest bei Patient/innen mit chronischer Niereninsuffizienz gezeigt [65]. Da die klassische Messung der carotis-femoralis PWV mittels Tonometrie im klinischen Alltag aufwändig ist, wurden Vereinfachungen entwickelt, unter anderem die Abschätzung der aortalen PWV mittels Oberarmmanschette [66]. Deren klinische Validierung in großen Endpunktstudien ist noch ausständig.

\section{Knöchel-Arm-Index}

Der Knöchel-Arm-Index (ankle brachial index - ABI) wird klassischerweise mit dem CW-Doppler und Blutdruckmanschetten (eine Verwendung automatischer Blutdruckmanschetten ist ebenfalls möglich) ermittelt als: $\mathrm{ABI}=$ systolischer $B D$ am Bein/systolischer $B D$ am Arm. Am Bein wird für die Formel der höhere BD an der A. dorsalis pedis oder der A. tibialis posterior herangezogen, am Arm der höhere $\mathrm{BD}$ an der rechten oder linken A. brachialis. Ein Wert $<0,9$ charakterisiert eine periphere arterielle Verschlusserkrankung (pAVK) und verdoppelt das im SCORE errechnete kardiovaskuläre 10-Jahres-Mortalitätsrisiko [67] und bewirkt eine Klassifizierung zu hohem bzw. sehr hohem kardiovaskulären Risiko.

\section{Die Niere bei arterieller Hypertonie}

Nach dem Diabetes mellitus ist die arterielle Hypertonie die zweithäufigste Ursache für eine Nierenfunktionsstörung. Auf der anderen Seite führen primäre Nie- renerkrankungen zur Entwicklung oder Verschlechterung einer Hypertonie.

Das Serum-Kreatinin ist initial ein insensitiver Parameter für eine Abnahme der Nierenfunktion, weshalb die eGFR errechnet werden sollte [68]. Eine Abnahme der eGFR unter $59 \mathrm{~mL} / \mathrm{min} / 1,73 \mathrm{~m}^{2}$ gilt als HVOS. Ein weiterer Parameter ist die EiweißAusscheidung im Harn, errechnet als Albumin-Kreatinin-Ratio in einer Einzel-Harn-Probe (vorzugsweise Morgenharn). Als Grenzwerte gelten: für die Mikroalbuminurie 30-300 mg/24h bzw. für die AlbuminKreatinin-Ratio 30-300 mg/g Kreatinin. Ein negativer Harn-Streifentest schließt eine Albuminurie nicht aus [69]. Sowohl verminderte eGFR als auch erhöhte Albumin/Kreatinin Ratio sind unabhängige Prädiktoren für kardiovaskuläre und renale Ereignisse [70].

Beide Parameter (eGFR, Albumin/Kreatinin Ratio) sollten bei Hypertonie zumindest einmal jährlich bestimmt werden. Liegen eine Nierenfunktionsstörung bzw. eine Albuminurie vor oder besteht der Verdacht auf eine sekundäre Hypertonie, sollte die Sonographie der Nieren bzw. die Doppleruntersuchung der Nierenarterien durchgeführt werden.

\section{Hypertensive Retinopathie}

Die Wertigkeit der hypertensiven Retinopathie ist als Prognosemarker gut abgesichert [71]. Mittels Fundoskopie werden schwere Veränderungen wie retinale Blutungen, Mikroaneursymen, harte Exsudate, „cotton wool spots“ und ein Papillenödem verlässlich und gut reproduzierbar nachgewiesen. Frühe Veränderungen wie Verengungen der Arteriolen oder arteriovenöse Kreuzungszeichen sind dagegen unzuverlässlich nachzuweisen (hohe inter-observer-Variabilität). Eine Fundoskopie sollte bei Blutdruckwerten $>$ 160/100 mm Hg und/oder Diabetes mellitus durchgeführt werden. Moderne Entwicklungen (Visualisierung des Augenhintergrunds mittels Smartphones) könnten in Zukunft an Bedeutung gewinnen. 


\section{Das Gehirn bei arterieller Hypertonie}

Die arterielle Hypertonie führt sowohl zu dramatischen Ereignissen wie Insult oder TIA als auch zu klinisch stummen Gehirnveränderungen, die man am besten mittels MRT nachweisen kann (sogenannte White Matter Lesions, Microbleeds oder kleine stille Mikroinfarkte, die zumeist lakunären Ursprungs sind) [72]. Ein besonders enger Zusammenhang sowohl der morphologischen als auch der strukturellen Veränderungen ist mit einer erhöhten Gefäß-Steifigkeit gegeben [73, 74]. Bildgebende Verfahren wie MRT oder CT sollten bei entsprechender Klinik wie kognitivem Abbau oder neurologische Symptome auftreten. Zusätzlich wird die (wiederholte) Durchführung von standardisierten Tests wie dem Mini-Mental-State zur Verlaufsbeobachtung bei klinischer Auffälligkeit empfohlen.

\section{Patientenselektion für das Screening von se- kundärer Hypertonie}

Sekundäre Hypertonie ist eine Bluthochdruckkrankheit, die durch eindeutig identifizierbare und potentiell behandelbare zu Grunde liegende Erkrankungen verursacht wird.

Es ist ein hohes Maß an Aufmerksamkeit erforderlich, um sekundäre Hypertonieformen frühzeitig erkennen zu können. Eine möglichst frühzeitige Diagnose ist allerdings erforderlich, um insbesondere bei jüngeren Patient/innen potentiell kurable Faktoren zu eliminieren und die Hypertonie noch vor dem Entstehen von Organschäden zu heilen.

$\mathrm{Zu}$ den potentiell korrigierbaren Ursachen zählen unter anderen die Coarctatio der Aorta, eine Nierenarterienstenose durch fibromuskuläre Dysplasie bei jüngeren Patient/innen, oder ein Nebennierenadenoms bei endokriner Hypertonie.
Zusätzlich kann eine Behandlung mit einem spezifischen Medikament bei monogenetischen Formen der arteriellen Hypertonie zu einer Blutdrucknormalisierung führen (z.B. Verwendung von Amilorid bei Liddle-Syndrom) [75].

Wenn der ideale Zeitpunkt der Korrektur einer sekundären Hypertonie verpasst wird, entstehen häufig Endorganschäden und es ist weniger wahrscheinlich, dass die Korrektur der zugrundeliegenden Ursache zu einer vollständigen Heilung (einschließlich Medikamentenfreiheit) bzgl. der arteriellen Hypertonie führt. Dennoch kann auch bei einer verzögerten Diagnose einer sekundären Hypertonie die Korrektur der zugrunde liegenden Ursache zu einer wesentlichen Verbesserung der Blutdruckeinstellung beitragen.

Die Prävalenz der sekundären Hypertonieformen wird in der Literatur zwischen 5 und 15\% der Hypertoniker/innen angegeben.

Ein Screening aller Patient/innen mit arterieller Hypertonie auf eine sekundäre Hypertonie, ist aufgrund der limitierten Sensitivitäten und Spezifitäten der für das Screening zur Verfügung stehenden diagnostischen Maßnahmen, weder sinnvoll noch kosteneffektiv. Allerdings sollten bestimmte Patient/ innencharakteristika dazu beitragen, die Schwelle für das Screening auf sekundäre Hypertonie zu reduzieren, sobald die Diagnose einer arteriellen Hypertonie (idealerweise mittels 24-h-Blutdruckmonitoring) gestellt ist.

$\mathrm{Zu}$ diesen Patient/innencharakteristika zählen ein jüngeres Manifestationsalter (unter 40 Jahre) mit deutlich ausgeprägter Hypertension (Grad 2 Hypertonie) oder das Auftreten einer arteriellen Hypertonie im Kindesalter. Auch eine sich rasch verschlechternde Hypertonie bei Patient/innen mit vorher dokumentierter Normotension oder eine resistente Hypertonie sowie sehr schwere Formen der Hypertonie einschließlich hypertensiver Notfälle sollten eine Di-
Tab. 10 Patientencharakteristika, die ein Screening auf sekundäre Hypertonie veranlassen können. (Adaptiert nach $[52,76,77])$

\footnotetext{
Jüngeres Manifestationsalter (unter 40 Jahre) mit deutlich ausgeprägter Hypertension (Grad II Hypertonie) oder das Auftreten einer arteriellen Hypertonie im Kindesalter

Plötzlicher Beginn und/oder rasch sich verschlechternde Hypertonie bei Patient/innen mit vorher dokumentierter Normotension

Resistente Hypertonie

Schwere Formen der Hypertonie einschließlich hypertensiver Notfälle [78]

Ausgedehnte Hypertonie-vermittelte Organschäden

Klinische oder biochemische Parameter, die auf eine endokrine Hypertonie (spontane oder Diuretika-induzierte Hypokaliämie, Episoden von Muskelschwäche und Tetanie - Hyperaldosteronismus; charakteristische Symptome einer Schilddrüsenüberfunktion, eines Hyperparathyroidismus oder eines Cushing-Syndroms) hinweisen

Klinische oder biochemische Parameter (Serum-Kreatinin, Harnsediment, Eiweißausscheidung im Harn), die auf eine chronische Nierenerkrankung hinweisen

Schwangerschaft oder Verwendung von Kontrazeptiva

Verwendung bestimmter Medikamente/Substanzen (Kokain, Amphetamine, Kortikosteroide, nasale Vasokonstriktoren, antineoplastische Chemotherapeutika, Yohimbine, Lakritze)

Klinische Zeichen eines obstruktiven Schlafapnoesyndroms (Schnarchen, nächtliche Atempausen, Tagesmüdigeit)

Symptome (wiederholte Episoden mit Schweißausbrüchen, Kopfschmerzen, Angstzuständen und Palpitationen; cafe-au-lait-Flecken) oder eine Familiengeschichte eines Phäochromocytoms

Abdominelles Strömungsgeräusch (Nierenarterienstenose) Blutdruckdifferenz zwischen oberen und unteren Extremitäten $(\geq 20 / 10 \mathrm{~mm} \mathrm{Hg})$
} 
agnostik auf sekundäre Hypertonieformen nach sich ziehen.

Zu den weiteren Triggern für die Suche nach sekundären Hypertonieformen, zählen ausgedehnte Hypertonie-vermittelte Organschäden, klinische oder biochemische Parameter, die auf eine endokrine Hypertonie oder chronische Nierenerkrankung hinweisen, klinische Zeichen eines obstruktiven Schlafapnoesyndroms und Symptome oder eine Familiengeschichte eines Phäochromocytoms (Tab. 10).

\section{Hypertonie und Nierenarterienstenose}

\section{Einleitung}

Atherosklerose ist bei circa 95 von 100 Patienten, eine fibromuskuläre Dysplasie bei etwa $5 \%$ der Betroffenen die Ursache einer Nierenarterienstenose (NAST). Sie gehört zu den häufigsten Ursachen einer sekundären Hypertonie, weshalb unter bestimmten Voraussetzungen gezielt danach gesucht wird [79]. Mit der Hoffnung, dass bei NAST mittels Intervention eine Heilung der Hypertonie möglich wird, kam es in den USA zu einem Anstieg von Dilatationen und Stentimplantationen bei NAST von 7660 im Jahr 1996 auf 18.520 Interventionen im Jahr 2000 [80] und die Zahl stieg bis 2005 auf geschätzt 35.000 Interventionen an [81].

Das Management einer NAST stellt nach wie vor eine Herausforderung für den Kliniker/die Klinikerin dar, zumal das Spektrum der Erkrankung von Zufallsbefunden [82] bis zu dramatischen klinischen Manifestationen wie dem blitzartig auftretenden Lungenödem [83] mit Intensivpflichtigkeit reichen kann. $\mathrm{Zu}$ bedenken ist auch noch, dass neben der Möglichkeit der renovaskulären Hypertonie eine ischämische Nierenerkrankung, das heißt Durchblutungsminderung aufgrund mikroangiopathischer Gefäßläsionen, vorliegen kann oder auch eine Kombination beider Krankheitsbilder.

Tab. 11 Anamnese und klinische Befunde, die an eine renovaskuläre Hypertonie denken lassen (sollten)

Schwere oder maligne Hypertonie (Endorganschäden!)
Behandlungsrefraktäre Hypertonie (mindestens 3 Antihypertensiva)
Plötzlicher Blutdruck-Anstieg bei vorher stabilen Werten
Hypertonie vor Pubertät
Hypertonie vor dem 30. Lebensjahr mit negativer Familienanamnese bezüg-
lich Hypertonie ohne Übergewicht
Kreatininanstieg >30\% vom Ausgangswert nach Gabe von ACE-Hemmern/
Angiotensin-Rezeptoren-Blockern
Hypertonie bei Patient/innen mit generalisierter Atherosklerose, einseitig
verkleinerter Niere oder Nieren-Größendifferenz >1,5cm
Hypertonie und wiederholt „flash pulmonary edema“
Beginn einer schweren Hypertonie erst nach dem 55. Lebensjahr [67]
Hypertonie und Strömungsgeräusch periumbilikal
Signifikant wechselnde Serum-Kreatinin-Werte im Verlauf, die in erster
Linie mit sich änderndem Volumenstatus in Zusammenhang stehen
Sich verschlechternde Nierenfunktion nach Implantation eines Aorten-Stent-
grafts

\section{Klinik und Diagnostik der NAST}

Klinische Symptome, die an eine NAST als sekundäre Hypertonie-Ursache denken lassen sollten sind in Tab. 11 angeführt. Eine NAST kann auch Ursache einer Nierenfunktionseinschränkung im Sinne einer ischämischen Nierenerkrankung sein, die klinischen Präsentationszeichen zeigt Tab. 12; [79, 84]. Die Abklärung sollte nur durchgeführt werden, wenn die Klinik an eine sekundäre Hypertonie denken lässt, wenn andere sekundäre Hypertonieformen wie eine Nephropathie, ein Hyperaldosteronismus oder Phäochromozytom unwahrscheinlich sind und vor allem wenn bei signifikanter NAST auch eine Intervention durchgeführt werden würde.

Ergibt sich aus Anamnese und klinischer Untersuchung, z. B. periumbilikales arterielles Strömungsgeräusch, ein konkreter Verdacht, so gibt es mehrere bildgebende Verfahren zur Diagnosestellung. Die Leitlinien der Europäischen Gesellschaft für Kardiologie in Zusammenarbeit mit der Europäischen Gesellschaft für Gefäßchirurgie stellen klar die Duplexsonographie an erste Stelle in der Diagnostik [67]. Sie beziehen sich damit ihrerseits auf Leitlinien der US Fachgesellschaften [85], aber auch andere Quellen [86, 87]. Die Methode ist nicht-invasiv und kann beliebig wiederholt werden, um etwa eine Progression mit Zunahme des Stenosegrades zu detektieren. Die Sensitivität von $85 \%$ und die Spezifität von $92 \%$ sind gut validiert (siehe Tab. 13), auch wenn der Stenosegrad möglicherweise etwas überschätzt wird [88]. Ergänzend kann die Bestimmung des renalen Widerstandsindex hilfreich sein $[87,89]$ Index-Werte $>80$ weisen auf eine geringe Ansprechrate auf Intervention hin. Nachteile der Duplexsonographie sind die Untersucherabhängigkeit, ev. schlechte Einsehbarkeit bei Übergewicht, eingeschränkte Beurteilbarkeit bei ev. nicht vollständiger Einsehbarkeit oder dass akzessorische Nierenarterien möglicherweise nicht entdeckt werden.

Auch die MR-Angiographie weist eine gute Sensitivität und Spezifität auf, tendiert aber ebenso zur Überschätzung des Stenosegrades. Allerdings werden damit akzessorische Nierenarterien sowie die Gefäße der Umgebung gut erfasst und eine Beurteilung der Nierenmorphologie und eine gewisse Beurteilung der exkretorischen Nierenfunktion ist möglich [90, 92]. Zu beachten ist, dass die Gabe von MR-Kontrastmittel bei

Tab. 12 Klinische Situationen, die an eine Nierenarterienstenose als Ursache einer Niereninsuffizienz denken lassen

Unerklärbare, persistierende und progrediente Niereninsuffizienz
Akute Verschlechterung einer chronischen Niereninsuffizienz (z. B. nach
ACE-Hemmer/Angiotensin-Rezeptor-Blocker-Gabe)
Stark schwankende Serum-Kreatinin-Werte, vermutlich aufgrund Schwan-
kungen im Volumenhaushalt
Niereninsuffizienz mit unauffälligem Harnsediment
Einseitig verkleinerte Niere


Tab. 13 Methoden zum Screening und zur Abklärung bei Verdacht auf Nierenarterienstenose

\begin{tabular}{|l|l|l|l|}
\hline Methode & Lit & Sensitivität (in \%) & Spezifität (in \%) \\
\hline Duplex-Sonographie & {$[88]$} & 85 & 92 \\
\hline \begin{tabular}{l|l|l} 
MR-Angiographie \\
[90]
\end{tabular} & 100 & 96 \\
\hline $\begin{array}{l}\text { Spiral-CT mit Angiogra- } \\
\text { phie }\end{array}$ & {$[91]$} & 93 & 81 \\
\hline
\end{tabular}

einer glomerulären Filtrationsrate $<30 \mathrm{ml} / \mathrm{min} / 1,73 \mathrm{~m}^{2}$ nicht empfohlen wird. Eine MR-Untersuchung ist aber auch ohne Kontrastmittel möglich. Liegt bereits ein Stenting locoregionär vor, so ist die Beurteilbarkeit durch Artefakte deutlich eingeschränkt.

Bei der Angiographie mittels Spiral-CT wird Kontrastmittel benötigt. Die üblichen Caveats sind zu beachten. Sensitivität und Spezifität der CT-Angiographie sind vergleichbar gut wie die MR-Angiographie $[91,93]$.

Goldstandard bleibt die invasive Angiographie mit arteriellem Gefäßzugang und Katheter und Darstellung in DSA-Technik (digitale Subtraktions-Angiographie) [93]. Dabei ist es mittels Druckmessungen vor und nach Stenose möglich, die hämodynamische Wirksamkeit einer NAST zu beweisen (Gradient $>20 \mathrm{~mm} \mathrm{Hg}$ ) und damit die Indikation für eine Intervention abzusichern [94]. Die konventionelle Angiographie soll erst zum Einsatz kommen, wenn aufgrund von Anamnese und Klinik eine HochrisikoSituation besteht, die eine Intervention rechtfertigen würde - siehe nachfolgend "Therapie einer NAST“.

Zusammenfassend geben die Europäischen Leitlinien für die Duplexsonographie als ,first-line'-Untersuchung, und in der Folge CT-Angiographie oder MRAngiographie zur weiteren Diagnosesicherung eine Klasse-I-Empfehlung mit einem Evidenzgrad ,B“ ab [67].

Weitere Hinweise hinsichtlich Diagnose und eventueller nachfolgender Intervention geben morphologische Aspekte wie der Nierendurchmesser oder das Organvolumen [95, 96]. Seitendifferenz mit kleinerer Niere auf der NAST-Seite und vermindertes Organvolumen ebendort sind diagnostische Hinweise, und haben auch eine gewisse prognostische Bedeutung. Ein nur gering verringertes Organvolumen scheint prognostisch günstig hinsichtlich des Ansprechens auf eine Intervention zu sein [96], während dies bei stark verkleinerter Niere nicht der Fall ist [95]. Zahlenmäßige Angaben, welche Größendifferenz relevant ist, gibt es leider nicht.

Wesentlich hinsichtlich der Prognose ist noch, dass die Lebenserwartung von Patient/innen mit NAST ohne terminale Niereninsuffizienz aufgrund erhöhter kardiovaskulärer Mortalität reduziert ist [97]. Bei Patient/innen mit Fortschreiten ihrer NAST-bedingten Niereninsuffizienz hin zu terminaler Niereninsuffizienz ist die Mortalitätsrate sogar noch höher [98].

\section{Therapie bei Nierenarterienstenose}

Medikamentöses Management

Medikamentöse Therapie und nicht-medikamentöse Therapiemaßnahmen, gemeint sind Lebensstilmaßnahmen, erfolgen nach den üblichen Empfehlungen für Patient/innen mit Atherosklerose [49, 99-101]. Alle Patient/innen sollen eine antihypertensive Therapie unter Einschluss eines ACE-Hemmers/ARBs [102, 103] erhalten. $\mathrm{Zu}$ beachten ist, dass ACE-Hemmer/ ARBs unter Umständen den glomerulären Perfusionsdruck so deutlich senken können, dass es zu einem markanten Kreatininanstieg, gelegentlich sogar zu einem akuten Nierenversagen kommen kann. Kontrollen des Serumkreatinins nach Therapiebeginn und im Verlauf sind daher angezeigt. Selbstverständlich sind die Dosierungsempfehlungen der Hersteller in Abhängigkeit vom Ausmaß der eventuell gleichzeitig vorhandenen Nierenfunktionseinschränkung zu beachten. Bei beidseitiger NAST oder wirksamer NAST und Einnierigkeit ist eine sorgfältige Nutzen-Risiko-Abwägung und kurzfristige Kontrolle der Nierenfunktionsparameter nach Therapiebeginn und bei jeder Dosisänderung zu beachten. Bei einzelnen ACE-Hemmern/ ARBs wird die beidseitige NAST als Kontraindikation angeführt. Weiter sind eine Thrombozytenaggregationshemmung und eine Statintherapie angezeigt. AuBerdem sollen alle Raucher/innen eine Beratung und Unterstützung zur Entwöhnung erhalten. Die Wirksamkeit dieses Ansatzes einer multimodalen Therapie wurde in der CORAL-Studie eindrucksvoll gezeigt, die medikamentöse Therapie war gleich wirksam wie die Intervention [104].

\section{Revaskularisation}

Eine Revaskularisierung kann unterschiedliche Ziele haben. Zum einen soll damit eine Verbesserung der Blutdrucksituation erreicht werden, zum anderen könnte bei ischämischer Genese eine Verbesserung der Nierenfunktion das Ziel sein.

Folgt man den zuletzt publizierten drei großen Studien zum Vergleich einer konservativen Therapie versus Intervention [104-106], so besteht im Allgemeinen keine Indikation für eine Revaskularisation mittels Dilatation und Stenting. Es zeigte sich kein Vorteil durch Intervention, das heißt der BD war nach Intervention im Vergleich zu medikamentöser Therapie nicht signifikant unterschiedlich und auch die Nierenfunktion wurde, sofern schon eingeschränkt, nicht verbessert.

Die möglichen Risiken der Katheterintervention bei atherosklerotischer NAST sind nicht zu unterschätzen, was sich in den größeren Studien zur Intervention bei NAST in Form beträchtlicher Nebenwirkungen (in $8 \%$ schwerwiegend) wie reno-renale Embolien, Nierenarterienperforationen, Nierenarterienverschlüsse, Cholesterinembolien mit Gangrän und Amputation, Herzinfarkt, akutes Nierenversagen, relevante Leistenhämatome und sogar kardiale Todesfälle zeigte [104-106]. Es waren allerdings viele Zentren mit teils 


\section{Hypertonie und/oder eingeschränkte Nierenfunktion}

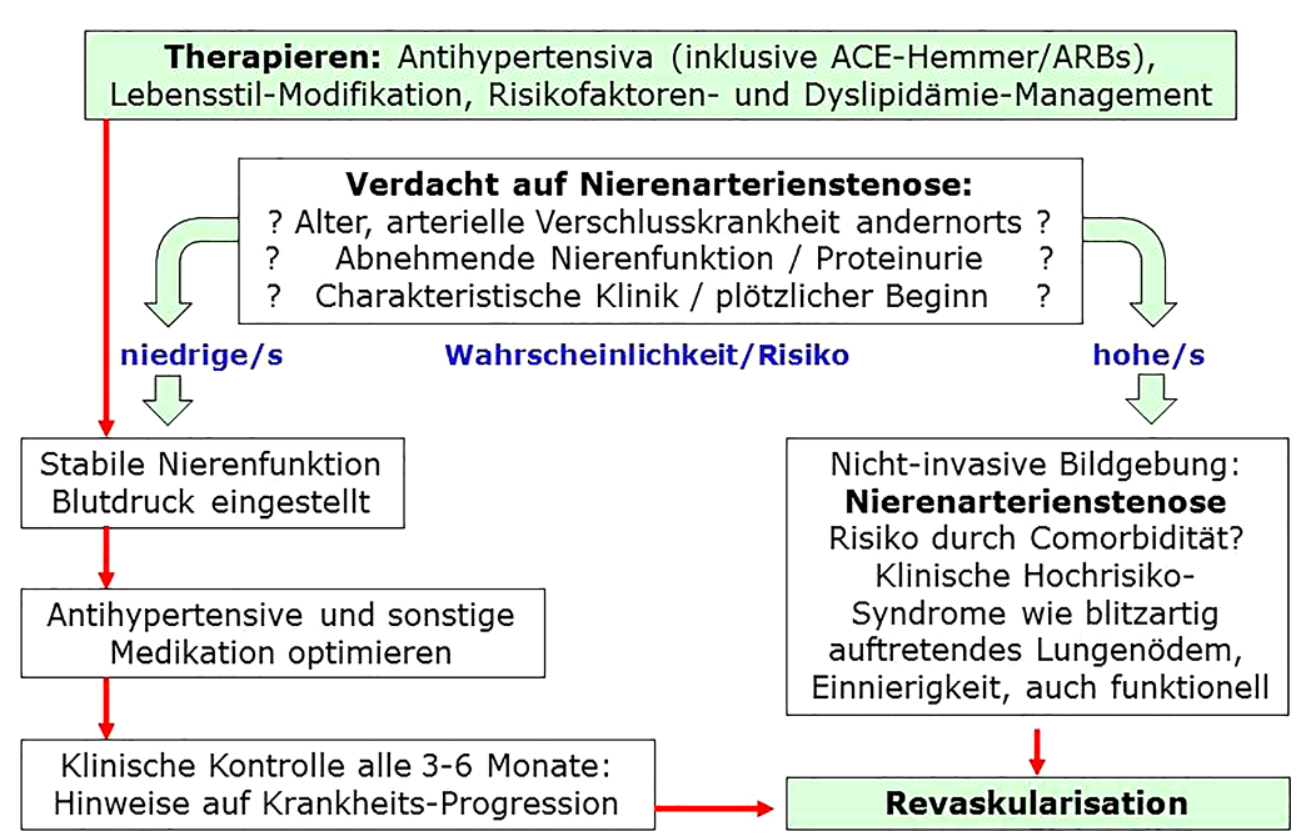

Abb. 3 Management der renovaskulären Hypertonie (bzw. ischämischen Nierenerkrankung). Bei klinischem Verdacht auf Nierenarterienstenose hängt die Vorgangsweise von Wahrscheinlichkeit und Risiko ab. Bei hoher Wahrscheinlichkeit ist die Diagnose mittels entsprechender Bildgebung zu sichern. Bei Hochrisiko-Patient/innen, das sind Patient/innen mit blitzartig auftretendem Lungenödem oder sich rasch verschlech-

nur geringer Fallzahl an diesen Studien beteiligt, weshalb die beträchtliche Komplikationsrate zumindest teilweise durch mangelnde Expertise erklärbar ist.

Dennoch sprechen Kohortenstudien dafür, dass unter bestimmten Umständen eine Intervention mit Dilatation und Stenting erwogen werden kann. Wie in Abb. 3 (adaptiert nach [107]) dargestellt, dürfte es Hochrisiko-Patient/innen geben, die von der Beseitigung der Stenose/n durch Intervention profitieren [108, 109]. Dies trifft insbesondere für Patient/innen $\mathrm{zu}$, die sich mit blitzartig auftretendem Lungenödem präsentieren, weiter Patient/innen mit kongestiver Herzinsuffizienz, insbesondere bei erhaltener linksventrikulärer Pumpfunktion, bei Patient/innen mit beidseitiger NAST, und für Patient/innen mit funktioneller/tatsächlicher Einzelniere und NAST [86, 108, $110]$.

\section{Besonderheiten bei Nierenarterienstenose durch Fibromuskuläre Dysplasie}

Die Prävalenz der Fibromuskulären Dysplasie (FMD) liegt bei $<1 \%$ in der Allgemeinbevölkerung, Frauen sind mit einer Ratio von 9:1 häufiger betroffen. Die renovaskuläre Erkrankung ist die häufigste klinische Manifestationsform der FMD, es gibt relevante Unterschiede zur atherosklerotisch bedingten NAST. Diagnostisch können die Gefäßveränderungen schwierig ternder Nierenfunktion (ohne andere Ursache) oder kongestiver Herzinsuffizienz mit erhaltener linksventrikulärer Pumpfunktion oder beidseitiger, hämodynamisch wirksamer NAST oder mit Einnierigkeit mit hämodynamisch wirksamer NAST oder mit medikamentös unkontrollierbarer Hypertonie, kann die Möglichkeit einer Revaskularisierung in Erwägung gezogen werden. (Adaptiert nach [107])

$\mathrm{zu}$ beurteilen sein, weil sie oft in die intrarenalen $\mathrm{Ab}$ schnitte der Nierenarterie reichen. Bei symptomatischer FMD und Zeichen der renalen Ischämie ist eine Intervention indiziert [111]. Empfohlen wird die Ballonangioplastie, eine zusätzliche Stentimplantation nur bei Gefäßwanddissektion oder Versagen der alleinigen Ballondilatation [112, 113]. Eine operative gefäßchirurgische Sanierung ist angezeigt, wenn gleichzeitig mit Stenosen komplexe Aneurysmen vorliegen, eine komplexe Gefäßanatomie, z.B. in Bifurkationsbereichen vorliegt, oder wenn die Intervention nicht wirksam war [113].

\section{Fact Box Nierenarterienstenose}

- Die durch Atherosklerose bedingte Nierenarterienstenose (NAST) ist die häufigste Ursache für renovaskuläre (sekundäre) Hypertonie, nur ca. $5 \%$ sind auf Fibromuskuläre Dysplasie zurückzuführen.

- Eine Abklärung hinsichtlich NAST soll nur erfolgen, wenn eine hohe Wahrscheinlichkeit besteht, dass ein Patient/eine Patientin von einer interventionellen Therapie profitiert (Hochrisiko-Patient/in).

- Besteht eine sekundäre Hypertonie renovaskulärer Genese, lässt sich aber der Blutdruck mit Standardtherapie gut einstellen, so ist eine Abklärung/ Intervention nicht indiziert. 
- Bei typischer klinischer Präsentation und Verdacht auf NAST sollte zur Diagnosestellung in erster Linie die Duplexsonographie verwendet werden.

- Alternative bildgebende Verfahren sind die MR-Angiographie oder die CT-Angiographie, wobei letztere eine Kontrastmittelgabe erfordert. Mit beiden kann die Diagnose gesichert werden.

- Bei atherosklerotischer NAST ist eine Revaskularisierung in der Mehrzahl der Betroffenen nicht indiziert. Sie verbessert weder den Blutdruck noch die Nierenfunktion und reduziert kardiovaskuläre Ereignisse nicht.

- Standard der medikamentösen Therapie bei atherosklerotischer NAST ist die Gabe von Antihypertensiva, Thrombozytenaggregationshemmern und Statinen und als nicht-medikamentöse Maßnahme das Beenden des Zigarettenrauchens (Beratung!).

- Hochrisiko-Patient/innen profitieren möglicherweise von einer Revaskularisierung, es bleibt aber eine individuell abzuwägende Entscheidung.

- Patient/innen mit symptomatischer Fibromuskulärer Dysplasie als NAST-Ursache profitieren von einer Revaskularisierung.

\section{Endokrine Hypertonie}

Die endokrine Hypertonie ist die häufigste sekundäre Hypertonieform mit einer Prävalenz von ca. 5-15\% bei Patient/innen mit arteriellem Hypertonus [52, 114-117]. Eine frühzeitige Diagnosestellung und Therapie von sekundären Hypertonieformen kann zu ei-

Tab. 14 Endokrine Hypertonieformen. (Adaptiert von [117])

\section{Adrenal bedingte Ursachen \\ 1. Primärer Hyperaldosteronismus \\ 2. Phäochromozytom/Paragangliom \\ 3. Cushing Syndrom \\ 4. Hyperdeoxycorticosteronismus}

(a) Adrenogenitales Syndrom (AGS) mit 11 $\beta$-Hydroxylase- oder 17-Hydroxylase-Mangel

(b) Deoxycorticosteron (DOC) produzierender Tumor

(c) Glukokortikoid-Resistenz

Apparenter Mineralokortikoid Exzess/11 $\beta$-Hydroxysteroid Dehydrogenase Mangel

1. Genetisch bedingt: Syndrom des apparenten Mineralokortikoidexzesses

2. Erworben: Lakritze, Cushing Syndrom (v. a. ektopes Cushing Syndrom)

Liddle Syndrom und Gordon Syndrom

Primärer Hyperparathyreoidismus

Hypophysär bedingte Ursachen

1. Akromegalie

2. Morbus Cushing

Sekundärer Hyperaldosteronismus: renovaskuläre Hypertonie, Renin produzierender Tumor

Schilddrüsenfunktionsstörungen

1. Hypothyreose

2. Hyperthyreose ner partiellen oder vollständigen Heilung der arteriellen Hypertonie führen und bewirkt folglich auch eine signifikante Reduktion von Morbidität und Mortalität [52, 114-117]. Bei den verschiedenen endokrinen Hypertonieformen (siehe Tab. 14) ist der primäre Hyperaldosteronismus (PHA) mit Abstand am häufigsten mit einer Prävalenz von ca. 5-10\% [52, 114-121].

Neben dem PHA hat hier auch das Phäochromozytom/Paragangliom als Leitsymptom einen arteriellen Hypertonus [122, 123]. Bei den meisten anderen endokrinen Hypertonieformen ist, abgesehen von seltenen genetischen Formen, die arterielle Hypertonie nur fakultativ bzw. als Begleitsymptom einer ansonsten anderweitig klinisch apparenten Erkrankung (wie. z. B. Cushing Syndrom) vorhanden [114, 115, 117]. Ein generelles Screening auf endokrine Hypertonieformen bei allen Patient/innen mit arterieller Hypertonie wird nicht empfohlen, sondern es sollte je nach Anamnese, Klinik bzw. Laborbefunden eine gezielte Diagnostik erfolgen [52, 114-121, 124].

\section{Primärer Hyperaldosteronismus (PHA)}

Der PHA (klassischerweise auch bekannt als Conn Syndrom) ist definiert „als eine Gruppe von Erkrankungen, bei denen die Aldosteron Sekretion unangemessen zu hoch im Bezug auf den Salz (Natrium) Status, relativ autonom von wichtigen Regulatoren der Aldosteron Sekretion (Angiotensin II und Kalium) und nicht supprimierbar durch Salzbelastung ist" $[118,119]$. Als häufigste endokrine Hypertonieform findet man diese Erkrankung bei ca. 5-10\% (in manchen Literaturstellen auch bis $20 \%$ ) aller Patient/ innen mit arterieller Hypertonie, wobei die Prävalenz mit steigenden BD-Werten zunimmt [118-121].

Patient/innen mit PHA haben im Vergleich zu primärer arterieller Hypertonie, selbst bei gleichen BDWerten, ein massiv erhöhtes kardiovaskuläres Risiko $[120,125,126]$. Da es beim PHA durch diverse Aldosteron Effekte (u.a. Natriumretention, BD Anstieg, etc.) zu einer Supprimierung des Renins kommt, re-

Tab. 15 Indikationen zur Abklärung auf einen primären Hyperaldosteronismus

Office-Blutdruck über 150/100 mm Hg bei drei Messungen an unterschiedlichen Tagen

Resistenter arterieller Hypertonus mit Office-Blutdruck über 140/90 mm Hg trotz Therapie mit 3 antihypertensiven Medikamenten (inklusive eines Diuretikums)

Kontrollierter arterieller Hypertonus (Blutdruck $<140 / 90 \mathrm{~mm} \mathrm{Hg}$ ) mit jedoch vier oder mehr antihypertensiven Medikamenten

Arterieller Hypertonus mit spontaner oder Diuretika induzierter Hypokaliämie

Arterieller Hypertonus bei Nebennieren-Inzidentalom

Arterieller Hypertonus bei Schlaf Apnoe Syndrom

Arterieller Hypertonus und Familienanamnese eines in jungen Jahren ( $<40$ Jahre) aufgetretenen arteriellen Hypertonus oder zerebrovaskulären Ereignisses

Alle hypertensiven erstgradig Verwandten eines Angehörigen mit primärem Hyperaldosteronismus 
Tab. 16 Auswahl wichtiger Faktoren für die Bestimmung und Interpretation der Aldosteron zu Renin Ratio (ARR). (Adaptiert von [119])

Hypokaliämie ausgleichen (cave: Hypokaliämie supprimiert Aldosteron; falsch negative ARR bei schwerer Hypokaliämie möglich)
Normale Kochsalzzufuhr
Folgende Medikamente sollten zumindest 4 Wochen vor der ARR Testung abgesetzt werden: Spironolacton
Eplerenon, Amilorid, Triamteren (ggf. passager ersetzen durch z. B. Doxazosin oder Verapamil)
Die Blutabnahme sollte am Vormittag nach $5-15$ min im Sitzen erfolgen, nachdem die Patientin/der Patient schon zumindest $2 \mathrm{~h}$ wach (aus dem Bett) ist (cave:
beim Hinlegen reduziert sich die ARR)
Verarbeitung der Proben bis zur Zentrifugierung bei Raumtemperatur (nicht im Eis); danach ggf. schnell einfrieren
Bzgl. Interpretation der ARR sind folgende Faktoren zu berücksichtigen:
Junge Frauen haben relativ häufig falsch erhöhte ARR Werte wegen einerseits oralen Kontrazeptiva (diese reduzieren Renin) und wegen der antimineralokortiko-
iden Wirkung des Progesterons (cave: 2 . Zyklushälfte, häufig erhöhte ARR)
Ältere Menschen und Patient/innen mit Niereninsuffizienz haben häufig höhere ARR
Diuretika, ACE-Hemmer und ARBs reduzieren die ARR (durch Reninanstieg) und Betablocker erhöhen die ARR (durch Reninabfall)
Es handelt sich um eine Auswahl verschiedener Faktoren für die Bestimmung und Interpretation der ARR, die bei weitem nicht vollständig ist

Tab. 17 Aldosteron-zu-Renin-Ratio (ARR) cut-off-Werte gemäß der Richtlinie der Endocrine Society [114]

\begin{tabular}{|c|c|c|c|c|}
\hline & Renin-Konzentration (mU/L) & Renin-Konzentration (ng/L) & Reninaktivität (ng/ml/h) & Reninaktivität (pmol/L/min) \\
\hline \multirow{3}{*}{$\begin{array}{l}\text { Aldosteron } \\
\text { (ng/dL) }\end{array}$} & $2,4^{\mathrm{a}}$ & 3,8 & 20 & 1,6 \\
\hline & 3,7 & 5,7 & 30 & 2,5 \\
\hline & 4,9 & 7,7 & 40 & 3,1 \\
\hline \multirow{2}{*}{$\begin{array}{l}\text { Aldosteron } \\
\text { (pmol/L) }\end{array}$} & 91 & 144 & 750 & 60 \\
\hline & 122 & 192 & 1000 & 80 \\
\hline
\end{tabular}

sultiert eine Erhöhung des Aldosteron zu Renin Quotienten (= Aldosteron zu Renin Ratio [ARR]), der somit auch als Screeningtest für diese Erkrankung empfohlen wird [118, 119]. Bei den in Tab. 15 aufgelisteten Konstellationen wird eine Diagnostik auf einen PHA mittels Bestimmung der ARR empfohlen, da bei diesen Patient/innen (v. a. auch in der Kombination Hypokaliämie und resistenter arterieller Hypertonus) die Vortestwahrscheinlichkeit für einen PHA deutlich erhöht ist [118, 119]. Eine Umsetzung dieser Empfehlungen bedeutet, dass in etwa bei jeder/m zweiten Patientin/Patienten mit arterieller Hypertonie eine ARR Bestimmung durchgeführt werden sollte. Dieses Screening wird jedoch in der klinischen Routine kaum umgesetzt, weswegen vermutlich über $90 \%$ der Patient/ innen mit PHA derzeit nicht diagnostiziert und somit auch weitgehend untherapiert sind [127-129].

Es gibt jedoch zahlreiche Faktoren, die neben der Labormethodik einen Einfluss auf die ARR haben, wobei in der klinischen Routine zu berücksichtigende Einflußgrößen auf die ARR in Tab. 16 aufgelistet sind.

Die prominenteste Empfehlung ist, dass Mineralokortikoid-Antagonisten (MRAs), z. B. Spironolacton, für zumindest 4 Wochen vor Bestimmung der ARR abgesetzt werden sollten, wohingegen in vielen Fällen die ARR trotz fortgeführter Medikamenteneinnahme (z. B. ACE-Hemmer oder Betablocker) oder anderer suboptimaler Bedingungen der Testung ausreichend interpretiert werden kann [118, 119]. Bei grenzwertigen Ergebnissen ist jedoch eine Wiederholung der Un- tersuchung nach Optimierung der Einflussfaktoren in Betracht zu ziehen.

In der Laboranalytik kann bei der Reninbestimmung entweder die Konzentration des Renins gemessen werden oder aber, was seltener durchgeführt wird und aufwendiger ist, die enzymatische Aktivität von Renin, welches als Protease das Angiotensinogen in das Angiotensin I umsetzt. Die Herausforderung bei der Interpretation der ARR liegt darin, dass es unterschiedliche cut-off Werte für eine positive/ pathologische ARR gibt, welche in Tab. 17 aufgelistet sind [118, 119]. Zusätzlich zur erhöhten ARR wird für einen positiven Screeningtest eines PHA teilweise auch gefordert, dass das Aldosteron im Plasma einen bestimmten Grenzwert überschreitet, wobei dbzgl. in der Literatur Werte von 6-15 ng/dL angegeben werden [119]. Jedes Zentrum ist somit gefordert, einen lokalen cut-off Wert in Hinblick auf die jeweilige Laboranalytik zu etablieren [119, 130].

Bei einer eindeutigen Laborkonstellation d.h. bei spontaner Hypokaliämie mit Plasma Aldosteron $>20 \mathrm{ng} / \mathrm{dL}$ und supprimiertem Renin (unter dem Detektionslimit des Assays) kann die Diagnose PHA gestellt werden [118, 119]. Bei pathologisch erhöhter ARR sollte ansonsten einer der in Tab. 18 angeführten Bestätigungsteste durchgeführt werden, wobei dies, sowie auch die weitere Abklärung, an einem Zentrum mit entsprechender Expertise durchgeführt werden sollte. 
Tab. 18 Bestätigungsteste für den primären Hyperaldosteronismus gemäß Endocrine Society guideline [119]

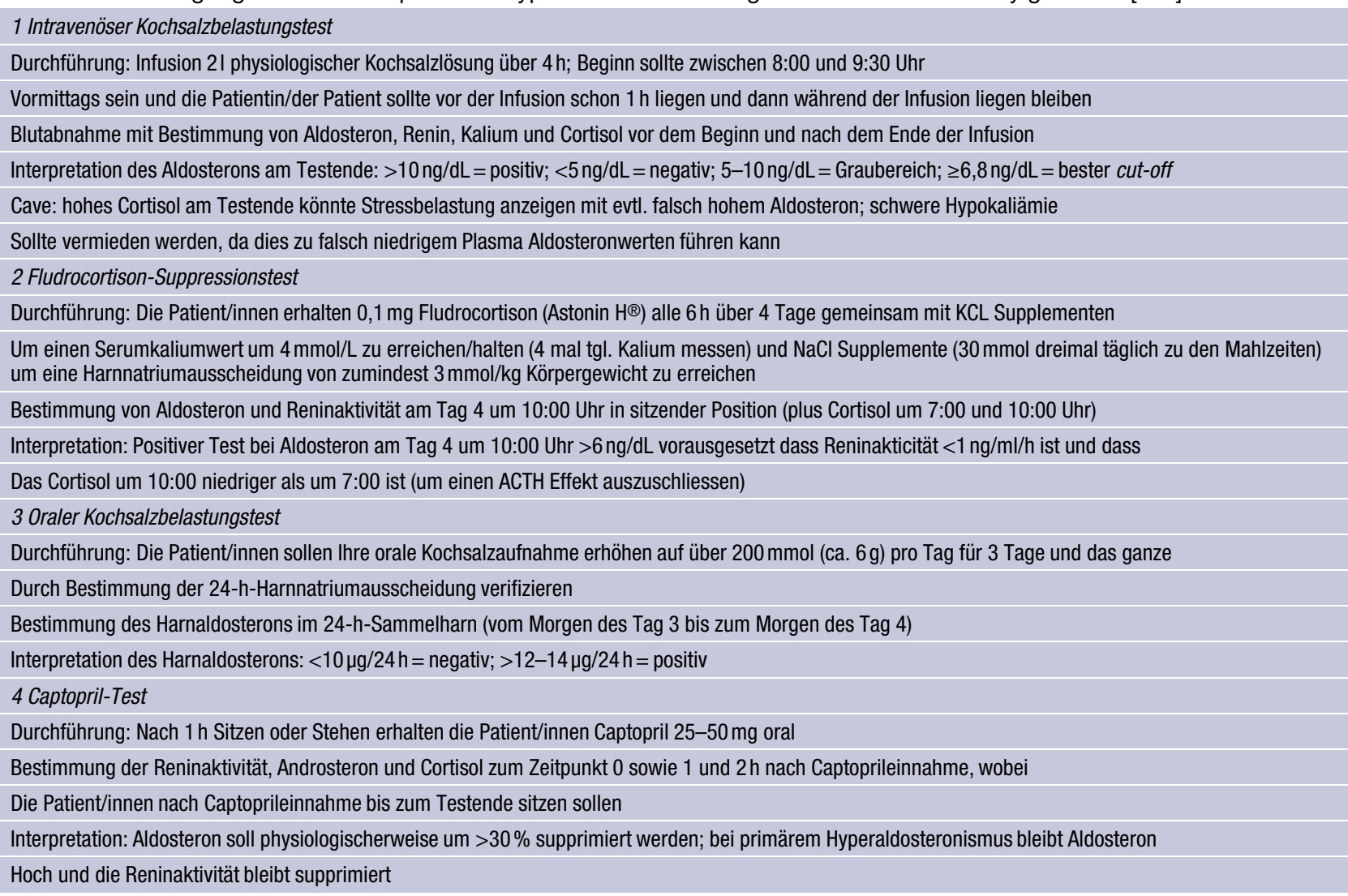

Bei positivem Bestätigungstest und somit biochemisch verifiziertem PHA wird gemäß Richtlinien zur Subtypenklassifizierung (siehe Tab. 19 bzw. Subtypen des PHA), vor allem zur Differenzierung des Aldosteron produzierenden Adenoms von der bilateralen adrenalen Hyperplasie sowie zur Diagnostik eines extrem seltenen Aldosteron sezernierenden Karzinoms, eine radiologische Bildgebung (in erster Linie Nativ CT) empfohlen, ggf. mit anschliessendem adrenalen Venensampling [118, 119]. Bzgl. Subtypenklassifizierung zeigte eine rezente randomisierte Studie jedoch keinen relevanten Unterschied im Outcome bei PHA

Tab. 19 Subtypen des primären Hyperaldosteronismus. (Adaptiert von [121])

\begin{tabular}{|l|l|}
\hline Aldosteron produzierendes Adenom (APA) & \multicolumn{2}{l}{ Prävalenz (\%) } \\
\hline Bilaterale (idiopathische) adrenale Hyperplasie (BAH) & 60 \\
\hline Primäre (unilaterale) adrenale Hyperplasie & 2 \\
\hline Aldosteron produzierendes Nebennierenkarzinom & $<1$ \\
\hline Familiärer Hyperaldosteronismus (FH) & \\
\hline $\begin{array}{l}\text { Glukokortikoid supprimierbarer Hyperaldosteronismus (FH } \\
\text { Typ 1) }\end{array}$ & $<1$ \\
\hline FH Typ 2 (BAH oder APA) & $<6$ \\
\hline FH Typ 3 (KCNJ5 Mutation) & $<1$ \\
\hline FH Typ 4 (CACNA1H Mutation) & $<0,1$ \\
\hline $\begin{array}{l}\text { Ektope Aldosteron produzierende Adenome oder Karzino- } \\
\text { me }\end{array}$ & $<0,1$ \\
\hline
\end{tabular}

Patient/innen die entweder gemäß des Ergebnisses des adrenalen Venensamplings oder gemäß des CT Befundes (OP-Indikation bei einseitiger adrenaler Raumforderung von $\geq 7 \mathrm{~mm}$ ) therapiert wurden [131].

In der Therapie des PHA wird bei Patient/innen mit beidseitiger adrenaler Erkrankung eine MRA-Therapie empfohlen. Dies wird auch bei Patient/innen mit PHA empfohlen, die unwillig oder untauglich für eine Operation sind bzw. auch bei Patient/innen mit erhöhter ARR, die eine weitere Abklärung/Untersuchung ablehnen. Als MRA wird primär Spironolacton empfohlen in einer Startdosis von 12,5-25 mg einmal täglich. Je nach klinischem Ansprechen kann diese Dosis schrittweise auf bis zu maximal $100 \mathrm{mg}$ pro Tag angehoben werden. Es sollten regelmäßig Kontrollen der Elektrolyte und des Kreatinins, z. B. innerhalb 1 Woche (bzw. besser noch innerhalb von 3 Tagen) und 4 Wochen nach Therapieeinleitung mit MRAs erfolgen. Eine Alternative zu Spironolacton ist Eplerenon, welches ein günstigeres Nebenwirkungsprofil hat und v. a. beim Auftreten einer Gynäkomastie unter Spironolacton mit einer Startdosis von $25 \mathrm{mg} \mathrm{1-0-1} \mathrm{tgl.} \mathrm{verschrieben} \mathrm{werden}$ kann. Evtl. kann auch, v. a. bei Nebenwirkungen auf MRAs, eine Therapie mit Amilorid verabreicht werden, welches den epithelialen Natriumkanal (ENaC) inhibiert, der auch das hauptsächliche Wirkungsziel der MRAs darstellt. In diesem Zusammenhang sei auch erwähnt, dass die PATHWAY-2-Studie gezeigt hat, dass bei resistentem arteriellen Hypertonus die blutdruck- 
senkende Wirkung von Spironolacton auch bei Patient/innen ohne PHA mit der ARR direkt korrelierte und Spironolacton sowie Amilorid anderen antihypertensiven Medikamenten überlegen war [132]. Bzgl. der medikamentösen Therapie des PHA gibt es zudem Hinweise, dass die Aufhebung einer Reninsuppression unter der Therapie mit MRAs indikativ ist für ein gutes Therapieansprechen [133].

Bei einseitigem PHA (meistens Aldosteron produzierendes Adenom, selten auch einseitige adrenale Hyperplasie), wird eine unilaterale Adrenalektomie empfohlen, da man dadurch eine lebenslange medikamentöse Therapie evtl. vermeiden kann, die vermutlich auch im Bezug auf harte klinische Endpunkte unterlegen ist. Präoperativ wird eine MRABehandlung empfohlen, um einerseits eine gute BDEinstellung und einen Kaliumausgleich zu erreichen, und um andererseits einen postoperativen Hypoaldosteronismus (durch präoperative Stimulierung der kontralateralen Nebenniere im Rahmen einer MRA-Therapie) zu vermeiden. MRAs sind (genauso wie Kalium Supplemente) postoperativ sofort abzusetzen. Postoperativ sollten regelmäßig Elektrolyt (Kalium) Bestimmungen erfolgen, und auch der BD sollte postoperativ engmaschig kontrolliert werden. In der PASO (Primary Aldosteronism Surgical Outcome)-Studie mit 705 Patient/innen mit unilateralem PHA hatten nach der Adrenalektomie $37 \%$ einen kompletten klinischen Therapieerfolg (= normotensiv ohne antihypertensive Medikamente) und $94 \%$ einen kompletten biochemischen Therapieerfolg [134]. Vor allem Frauen und jüngere Patient/innen hatten eine sehr hohe Wahrscheinlichkeit für einen kompletten klinischen Therapieerfolg.

\section{Primärer Hyperaldosteronismus (PHA) - Fact Box}

- Der PHA ist die häufigste sekundär endokrine Hypertonieform mit einer Prävalenz von ca. 5 bis $10 \%$

- Eine Abklärung auf PHA ist bei ca. jedem zweiten Patient/innen mit Hypertonie indiziert, v. a. bei resistentem arteriellem Hypertonus und/oder Hypokaliämie

- Als Screeningtest wird die Bestimmung der Aldosteron zu Renin Ratio empfohlen

- Diagnosesicherung erfolgt in der Regel durch einen Bestätigungstest (z.B. i.v. Kochsalzbelastungstest) mit nachfolgender Bildgebung (in erster Linie CT) der Nebennieren

- Therapiert wird der PHA bei einseitiger Erkrankung (meist Nebennierenadenom) mittels Adrenalektomie und ansonsten mittels Mineralokortikoid-RezeptorAntagonist-Therapie

\section{Phäochromozytom/Paragangliom}

Phäochromozytome sind Tumore von chromaffinen Zellen des Nebennierenmarks, während Paragangliome von extraadrenalen chromaffinnen Zellen abstammen. Im Gegensatz zum PHA ist das Phäo-
chromozytom/Paragangliom (Anmerkung: nachfolgend wird zur Vereinfachung statt Phäochromozyom/ Paragangliom nur der Begriff Phäochromozytom verwendet) eine sehr seltene Erkrankung mit einer Prävalenz bei ambulanten Patient/innen mit arteriellem Hypertonus von ca. $0,2-0,6 \%$ [122]. Die klinische Symptomatik kann sehr heterogen sein, wobei die klassische klinische Trias aus Kopfschmerzen, Schwitzen und Palpitation nur bei ca. $10 \%$ der Patient/ innen zu beobachten ist. Die Phäochromozytom Patient/innen sind nicht immer hypertensiv, sondern können teils auch durch orthostatische Hypotonie symptomatisch sein (siehe Tab. 20 für die Symptome/ Klinik bei Phäochromozytom) [122, 123].

Phäochromozytome werden häufig zu Lebzeiten nicht diagnostiziert und verlaufen unbehandelt in der Regel tödlich. Eine Abklärung auf Phäochromozytom wird bei jeder Patientin/jedem Patienten mit entsprechender/verdächtiger Klinik, v. a. bei paroxysmal auftretenden Symptomen empfohlen, bei jedem Nebenniereninzidentalom, bei hereditärer Prädisposition für ein Phäochromozytom (z.B. von-HippelLindau-Syndrom), sowie bei medikamentös induzierten Blutdruckentgleisungen bei z.B. Narkose [122, 123]. Die Schwelle zur diagnostischen Abklärung auf ein Phäochromozytom sollte, v. a. aufgrund des fatalen unbehandelten Verlaufes der Erkrankung, niedrig sein [122, 135].

Bzgl. der Phäochromozytomabklärung können entweder die fraktionierten Metanephrine (d.h. getrennte Bestimmung von Metanephrin und Normetanephrin) im Plasma oder im 24-h-Sammelharn verwendet werden [10]. Die Bestimmung im Plasma oder im Harn wird als gleichwertig angesehen, wobei vielerorts, die meistens praktisch leichter durchführbare Bestimmung im Plasma präferiert wird. Zum besseren Verständnis der Diagnostik sei erklärt, dass Metanephrine und Normetanephrine Stoffwechselmetabolite von Adrenalin und Noradrenalin sind, welche grundsätzlich kontinuierlich (und nicht abhängig von der teils episodischen exozytotischen Freisetzung von Ad-

Tab. 20 Klinik bei Phäochromozytom (Angaben in \%)

\begin{tabular}{|l|}
\hline Arterieller Hypertonus $80-90$ \\
\hline Davon permanent $50-60$ \\
\hline Davon intermittierend (paroxysmal) 30 \\
\hline Kopfschmerzen $60-90$ \\
\hline Schwitzen $55-75$ \\
\hline Palpitationen $50-70$ \\
\hline Blässe 40-45 \\
\hline Hyperglykämie 40 \\
\hline Müdigkeit 25-40 \\
\hline Übelkeit 20-40 \\
\hline Gewichtsverlust 20-40 \\
\hline Angst, Panik 20-40 \\
\hline Orthostatische Hypotonie 10-50 \\
\hline Flush 10-20 \\
\hline
\end{tabular}


renalin und Noradrenalin) von den Tumoren durch das dort Membran gebundene Enzym Catecholamine O-Methlytransferase (COMT), gebildet werden [122]. Etwa die Hälfte aller Phäochromozytome produzieren einen Mix aus Noradrenalin und Adrenalin, während die andere Hälfte fast ausschliesslich Noradrenalin oder Noradrenalin und Dopamin produzieren (Anmerkung: Paragangliome produzieren fast ausschliesslich Noradrenalin oder Noradrenalin und Dopamin; in extrem seltenen Fällen nur Dopamin, welches gemeinsam mit seinem Metabolit Methoxytyramin gemessen werden kann) [122].

Für die Bestimmung der Plasma Metanephrine und/oder Normetanephrine wäre es optimal, wenn die Blutabnahme nach zumindest 30-minütigem Liegen erfolgt. Da dies organisatorisch oft nur schwer möglich ist, kann aus pragmatischer Sicht auch eine initiale Bestimmung der Plasma Metanephrine/ Normetanephrine im Sitzen erfolgen. Da im Sitzen die Metanephrin/Normetanephrin Werte im Plasma grundsätzlich höher sind als im Liegen, kann bei „negativen“ Metanephrinen/Normetanephrinen im Sitzen ein Phäochromozytom ausgeschlossen werden. Andererseits sollte bei erhöhten Plasma Metanephrinen/Normetanephrinen im Sitzen eine Testwiederholung nach zumindest $30 \mathrm{~min}$ im Liegen erfolgen, um falsch positive Ergebnisse zu minimieren. Falsch positive Testergebnisse mit erhöhten Metanephrinen/Normetanephrinen können auch durch Stress (z.B. im Rahmen einer akuten Erkrankung) sowie durch Medikamente bedingt sein (z.B. trizyklische Antidepressiva, selektive Serotonin Reuptake Inhibitoren [SSRI], selektive Noradrenalin Reuptake Inhibitoren [SNRI], Monoaminoxidase Inhibitoren, etc.; Anmerkung: bei positivem Screeningtest immer auch Medikamentenliste zwecks Einfluss auf Laboranalytik überprüfen) [122, 136]. Nikotinkonsum und Kaffee können evtl. auch zu falsch positiven Ergebnissen führen und sollte vor der Blutabnahme pausiert werden [136]. Die fraktionierten Metanephrine sind hierbei sehr sensitiv (d.h. ein negatives Testergebniss bedeutet mit hoher Sicherheit einen Ausschluss der Erkrankung), aber leider nicht sehr spezifisch mit häufig falsch positiven (leicht) erhöhten Werten. Diesbezüglich sollte so vorgegangen werden, dass bei einem positiven Test, bei dem Plasma Metanephrin und/oder Normetanephrin erhöht aber weniger als 3-fach über dem oberen Grenzbereich liegt, eine Testwiederholung unter optimalen Bedingungen erfolgen sollte (z. B. im Liegen und ohne vorangehendem Rauchen; evtl. optimierte Medikation). Sind dann die Metanephrine und Normetanephrine im Normbereich, kann ein Phäochromozytom ausgeschlossen werden. Bei noch immer (leicht) erhöhten Plasma Metanephrinen/Normetanephrinen sollte in erster Linie ein Clonidinhemmtest durchgeführt werden (alternativ eine Kombination aus Chromogranin A und 24-h-Harn-Metanephrinen). Bei einem Clonidinhemmtest, welcher in liegender Position durchgeführt wird, erfolgt nach initialer Blutabnahme zur Bestimmung von Normetanephrin und Noradrenalin die orale Verabreichung von $300 \mu \mathrm{g}$ Clonidin $(300 \mu \mathrm{g}$ pro $70 \mathrm{~kg}$ Körpergewicht), und nach weiteren $180 \mathrm{~min}$ die nochmalige Bestimmung von Normetanephrin (sollte zum Ausgangswert über $40 \%$ abfallen) und Noradrenalin (sollte über $50 \%$ abfallen). Wenn jedoch schon bei einer einzigen Blutabnahme (selbst unter suboptimalen) Bedingungen (oder bei einer Bestimmung im 24-h-Sammelharn) die Metanephrine und/oder Normetanephrine 3-fach oder mehr über dem oberen Grenzbereich liegen, kann man grundsätzlich die biochemische Diagnose eines Phäochromozytoms als gesichert ansehen und es sollte eine weitere bildgebende Diagnostik erfolgen. Bei sehr grossen (z. B. über $5 \mathrm{~cm}$ grossen) oder bilateralen Phäochromozytomen sollte (zusätzlich zur initialen Bildgebung welche in erster Linie ein CT ist) eine ${ }^{123}$ Jod-MIBG(Metaiodbenzylguanidin-)Szintigraphie durchgeführt werden, um eine evtl. Metastasierung auzuschliessen bzw. zu detektieren. Bei bereits radiologisch vorbekannten Metastasen im z.B. CT wird initial ein ${ }^{18} \mathrm{~F}-\mathrm{FDG}$ PET/CT als bevorzugte Bildgebung empfohlen. Malignität ist beim Phäochromozytom übrigens durch eine vorhandene Metastasierung definiert (ca. 10-17\% aller Phäochromozytome sind maligne).

Bei Diagnosestellung eines Phäochromozytoms sollte auch immer eine genetische Testung erwogen (angeboten) werden, da auch ca. jeder 3. Patient bzw. Patientin eine genetische Mutation mit Prädisposition für ein Phäochromozytom aufweist (z. B. Multiple Endokrine Neoplasie Typ 2; Neurofibromatose Typ 1; von-Hippel-Lindau-Syndrom; etc). Es wird dabei je nach vorliegenden Befunden (z. B. je nach Lokalisation, Vorhandensein von Metastasen, Dominanz von entweder Normetanephrin oder Metanephrin, Klinik eines Syndroms etc.) eine sequentielle genetische Testung durchgeführt [122].

Ein nicht metastasiertes Phäochromozytom wird meistens durch eine komplette Adrenalektomie therapiert. Präoperativ empfehlen die Richtlinien eine zumindest 7 - bis 14-tägige Therapie mit einem Alphablocker, d.h. Phenoxybenzamin mit einer üblichen Startdosis von $10 \mathrm{mg}$ 1-0-1 tgl. (alternative z. B. Doxazosin beginnend mit $2 \mathrm{mg} / \mathrm{Tag}$ ). Phenoxybenzamin sollte dann je nach Klinik/Blutdruck auf eine Zieldosis von $1 \mathrm{mg} / \mathrm{kg} /$ Tag präoperativ hinauftitriert werden (z.B. Steigerung um ca. $10 \mathrm{mg} / \mathrm{Tag}$; Cave: auf orthosthatische Dysregulation achten). Falls die Alpha-Blocker-Therapie nicht ausreichend ist, werden zusätzlich Kalziumkanal-Blocker empfohlen (z. B. Amlodipin). Eine Beta-Blocker-Therapie kann zur Tachykardie-Kontrolle indiziert sein, sollte aber erst nach ausreichender Alpha-Blocker-Therapie eingeleitet werden, da ansonsten paradoxe Blutdruckanstiege induziert werden könnten (cave: überwiegende Vasokonstriktion aufgrund zu geringer Alpha-Blockade). Postoperativ sollte v. a. in den ersten $24-48 \mathrm{~h}$ eine regelmässige Kontrolle von BD, Herzfrequenz und Blut- 
zucker (cave: Hypoglykämierisiko) erfolgen, wobei v. a. die postoperative Hypotonie sehr gut auf Volumsgabe anspricht. Ca. 2-4 Wochen nach der Adrenalektomie sollte eine follow-up-Untersuchung erfolgen, um die erfolgreiche Resektion des Phäochromozytoms auch biochemisch $\mathrm{zu}$ verifizieren (z.B. Bestimmung der fraktionierten Plasma Metanephrine). Danach sind lebenslange (zumindest) jährliche endokrinologische Kontrollen empfohlen.

\section{Phäochromozytom - Fact Box}

- Das Phäochromozytom ist eine seltene, aber ohne Therapie in der Regel tödlich verlaufende sekundäre Hypertonieform

- Eine Diagnostik auf Phäochromozytom ist bei verdächtiger Klinik z.B. paroxysmale Symptome z.B. RR Anstiege mit Kopfschmerzen, Schwitzen und Palpitationen indiziert wobei die Schwelle zur Abklärung niedrig angesetzt werden sollte

- In der Diagnostik wird die Bestimmung der Normetanephrine und Metanephrine im Plasma oder $24 \mathrm{~h}$ Harn empfohlen (beide Bestimmungsmethoden sind gleichwertig)

- Nach biochemischer Diagnosestellung ist eine abdominelle Bildgebung indiziert und es sollte eine genetische Untersuchung erfolgen bzw. angeboten werden

- Therapiert wird das Phäochromozytom mittels Operation wobei eine präoperative Vorbereitung mit Phenoxybenzamin (Dibenzyran ${ }^{\circledR}$ ) (alternative z.B. Doxazosin [Supressin ${ }^{\circledR}$ ]) für 1-2 Wochen erfolgen sollte

\section{Hypercortisolismus - Cushing Syndrom}

Das endogene Cushing Syndrom/Hypercortisolismus ist eine sehr seltene Erkrankung (Inzidenz nur ein paar Fällen pro Millionen Einwohner pro Jahr), die durch einen Glukokortikoidhormonexzess charakterisiert ist. Meistens liegt ein sogenannter Morbus Cushing, d.h. ein ACTH produzierender Hypophysentumor, vor. In selteneren Fällen handelt es sich um einen Cortisol produzierenden Nebennierentumor bzw. in manchen v. a. klinisch ausgeprägten Fällen um einen ektopen ACTH produzierenden Tumor (z.B. Bronchialkarzinom) [137-141]. Patient/innen mit klassischem Cushing Syndrom haben eine deutlich erhöhte Mortalität. Die häufigsten Todesursachen sind kardiovaskuläre Erkrankungen, venöse Thrombosen (Thrombembolien) und Infektionen. Beim Cushing Syndrom steht jedoch der arterielle Hypertonus im Vergleich zu anderen Symptomen/klinischen Stigmata des Cushing Syndroms eher im Hintergrund. Eine Diagnostik auf einen Hypercortisolismus sollte nur bei ausgeprägtem klinischen Verdacht durchgeführt werden. Typische Cushing Stigmata wären hierbei z. B. faziale Plethora (Rötung), Neigung zu Blutergüssen/ Hautblutungen, proximale Myopathie, Striae wenn über $1 \mathrm{~cm}$ breit und livide/rötlich. Andere mögliche
Charakteristika sind eine auffallend zentrale Adipositas mit vergleichsweise dünnen Extremitäten, eine rundliche Gesichtsform (Vollmondgesicht), zigarettenpapierartige dünne Haut, sowie weiters auch evtl. schlechte Wundheilung, Hirsutismus, Akne, Osteoporose bzw. in extrem ausgeprägten Fällen von v.a. ektopem Cushing Syndrom eine massiv ausgeprägte Hypokaliämie mit metabolischer Alkalose. Bzgl. der genauen Diagnostik und Therapie des Cushing Syndroms wird aufgrund der eher geringen Bedeutung bei der arteriellen Hypertonie auf die entsprechende Fachliteratur verwiesen [137-141].

\section{Seltene endokrine Hypertonieformen}

Bei folgenden seltenen endokrinen Hypertonieformen wird nur ein kurzer Überblick gegeben wobei bzgl. genauerer Diagnostik und Therapie auf die entsprechende Fachliteratur verwiesen wird. Bei jungen Patient/innen mit der Konstellation arterielle Hypertonie, Hypokaliämie und niedriges Aldosteron und Renin sollte man differentialdiagnostisch vor allem an einen Hyperdeoxycorticosteronismus denken, der durch einen Überschuss von Nebennierenhormonen wie v.a. Deoxycorticosteron (DOC) ausgeprägte mineralokortikoide Wirkungen hervorruft [117, 142, 143]. Die zu einem Hyperdeoxycorticosteronismus führenden Erkrankungen sind das adrenogenitales Syndrom (AGS) (21-Hydroxylase- oder 11 $\beta$-HydroxylaseMangel), ein DOC produzierender Tumor, sowie eine primäre Glukokortikoid-Resistenz, auch ChrousosSyndrom genannt.

Bei einem apparenten Mineralokortikoid Exzess (AME) handelt es sich um ein Syndrom mit fehlender oder eingeschränkter Aktivität des Enzyms 11ßHSD2, welches charakterisiert ist durch die Trias arterielle Hypertonie, Hypokaliämie und supprimiertes Aldosteron und Renin [117, 144, 145]. In Aldosteron-Zielorganen führt 11ßHSD2 u.a. zu einer „Inaktivierung“ von Cortisol zu Corticosteron, wodurch der Mineralokoirtikoid-Rezeptor durch Aldosteron und nicht durch das wesentlich höher konzentrierte Cortisol aktiviert werden sollte. Eine reduzierte 11ßHSD2-Aktivität führt somit zu einer überschiessenden Aktivierung des Mineralokoirtikoid-Rezeptor durch Cortisol. Der AME kann genetisch bedingt sein, wobei ein AME auch erworben sein kann durch z. B. übermässigen Konsum von Lakritze oder Carbenoxolon, welche die 11ßHSD2 inhibieren [146].

Das Liddle Syndrom ist eine seltene autosomal dominant vererbte Erkrankung mit aktivierender Mutation des epithelialen Natriumkanals (ENaC) [147-149]. Betroffene haben einen in jungen Jahren auftretenden arteriellen Hypertonus, eine Hypokaliämie, metabolische Alkalose, sowie niedriges Aldosteron und Renin (Anmerkung: eine ENaC Aktivierung ist ein klassischer Mineralokortikoid-Rezeptor mediierter Effekt).

Das Gordon Syndrom ist ebenfalls eine seltene meistens autosomal dominant vererbte Erkrankung 
mit Hyperkaliämie sowie supprimiertem Renin und relativ niedrigem Aldosteron. Beim Gordon Syndrom kommt es durch verschiedene Mutationen $\mathrm{zu}$ einer Aktivierung des Thiazid sensitiven $\mathrm{Na} / \mathrm{Cl}$ Cotransporters (NCC) sowie verminderten Expression des „Renal Outer Medullary Potassium Channels“ (ROMK).

\section{Primärer Hyperparathyreoidismus}

Ein primärer Hyperparathyreoidismus (Prävalenz fast $1 \%)$ ist typischerweise charakterisiert durch eine Hyperkalzämie mit erhöhtem oder unangemessen zu hohem Parathormon (PTH), was meistens auf ein (oder mehrere) Nebenschilddrüsenadenome zurückzuführen ist [150, 151]. Diese Erkrankung kann u.a. zu Urolithiasis, eingeschränkter Nierenfunktion und Osteoporose führen. Ein Zusammenhang mit arterieller Hypertonie und erhöhten kardiovaskulärem Risiko wurde auch beschrieben, die Evidenzlage dazu ist aber teilweise inkonsistent [152]. Bei biochemischem Nachweis eines primären Hyperparathyreoidismus (Kalzium und PTH hoch) erfolgt dann eine Bildgebung der Nebenschilddrüsen in erster Linie mit Sonographie. Therapiert wird ein primären Hyperparathyreoidismus in der Regel mittels Nebenschilddrüsenoperation [150, 151].

\section{Hypophysär bedingte Ursachen}

Eine Akromegalie (Prävalenz ca. 2,8-13,7 Fälle pro 100.000) wird durch erhöhte Werte des Wachstumshormons sowie des Insulin-like-growth-Factors-1 (IGF-1) verursacht und ist meistens auf ein Wachstumshormon sezernierendes Hypophysenadenom zurückzuführen [153-155]. Typische Charakteristika sind Riesenwuchs (im Kindesalter) sowie vergrösserte Akren mit u.a. auch zunehmender Schuhgröße im Erwachsenenalter, nicht mehr passender Ring, Karpaltunnelsyndrom etc; dbzgl. oft ein Gesichtsvergleich mit alten Fotos diagnostisch wegweisend. Darüber hinaus kann eine Akromegalie neben einem Diabetes mellitus und einer Cardiomyopathie regelmässig auch zu einer arteriellen Hypertonie führen [153, 155, 156]. Als Screeningtest wird die IGF-1-Bestimmung im Serum empfohlen mit bei erhöhten Werten weiterer Spezial-Abklärung in einem dbzgl. Zentrum. Therapeutisch wird eine Akromegalie operativ (Hypophysenoperation) behandelt [153, 155].

Das Cushing Syndrom, welches meistens als Morbus Cushing durch ein Hypophysenadenom bedingt ist, wurde bereits weiter oben ausgeführt. Ein exogener Hypercortisolismus durch eine Glukokortikoidtherapie ist jedoch wesentlich häufiger als der endogene Hypercortisolismus.

\section{Sekundärer Hyperaldosteronismus}

Eine sekundärer Hyperaldosteronsimus liegt typischerweise bei einer renovaskulären Hypertonie vor, bei der aufgrund einer Nierenarterienstenose eine konsekutive Aktivierung des Renin Angiotensin Aldosteron Systems vorliegt [157].

Ein Renin produzierender Tumor (auch Reninom oder Tumor des Juxtaglomerulären Apparates genannt) kann über eine vermehrte Reninproduktion zu einem Hyperaldosteronismus mit Hypertonie und Hypokaliämie sowie metabolischer Alkalose führen [45, 46]. Neben der Bildgebung kann auch ein renales Venensampling mit Bestimmung des Renins aus beiden Nebennierenvenen diagnostisch hilfreich sein [158, 159]. Obwohl die Patient/innen gut auf Renin-Angiotensin-Aldosteron-blockierende Medikamente ansprechen ist, die operative Tumorentfernung grundsätzlich die Therapie der Wahl [158, 159].

\section{Schilddrüsenfunktionsstörungen}

Schilddrüsenhormone haben weitreichende Wirkungen auf das Herz-Kreislaufsystem, wobei sowohl eine Hypothyreose als auch eine Hyperthyreose zu erhöhten BD-Werten führen können [160-163]. Eine laborchemische Bestimmung von TSH und Thyroxin (fT4) sollte bei klinischem Verdacht auf eine Schilddrüsenfunktionsstörung erfolgen. Bei einer subklinischen Hypothyreose mit erhöhtem TSH und im Normbereich gelegenem Thyroxin (fT4) zeigte sich in einer rezenten Meta-Analyse ein blutdrucksenkender Effekt einer LT4-Therapie von $-4,80 \mathrm{mmHg}$ (95\% CI: $-6,50$ bis $-3,09 ; p<0,001)$ auf den systolischen $\mathrm{BD}$ und $-2,74 \mathrm{~mm} \mathrm{Hg}$ (95\% CI: $-4,06$ bis $-1,43 ; p<0,001$ ) auf den diastolischen BD. Bezüglich Diagnostik und Therapie von Schilddrüsenfuktionsstörungen wird auf die entsprechenden Richtlinien verwiesen [164-167].

\section{Conclusio}

Die korrekte Diagnosestellung sekundär endokriner Hypertonieformen ermöglicht in vielen Fällen eine kausale Therapie, welche konsekutiv auch $\mathrm{zu}$ einer deutlich reduzierten Morbidität und Mortalität führt. Der PHA ist die häufigste endokrine Hypertonieform, wird jedoch aktuell massiv unterdiagnostiziert und somit untertherapiert. Viele der anderen endokrinen Hypertonieformen sind zwar sehr selten, sollten aber bei entsprechendem klinischen Verdacht einer endokrinologischen Diagnostik zugeführt werden.

\section{Nichtmedikamentöse Blutdrucksenkung}

Alle Hochdruckpatient/innen sollten über die Möglichkeiten der nicht medikamentösen Blutdrucksenkung informiert werden. Ein gesunder Lebensstil kann das Auftreten einer Hypertonie verzögern oder gar verhindern [49]. Effektive Lebensstilmaßnahmen können bei Grad 1 Hypertonie die Notwendigkeit einer medikamentösen Therapie hinauszögern oder gar erübrigen, bzw. im Falle einer medikamentösen Therapie die Effizienz derselben verstärken. Die Durchfüh- 
rung von Lebensstilmaßnahmen oder das Vorhaben, solche zu beginnen, darf die Einleitung einer medikamentösen Therapie bei Vorliegen eines hohen kardiovaskulären Risikos (z. B. bei Vorliegen von HVOS) aber nicht verzögern. Ein Nachteil von Lebensstilmaßnahmen ist, dass sie von Patient/innen häufig nicht über einen langen Zeitraum aufrechterhalten werden können [168].

\section{Natrium- und Kochsalzrestriktion}

Eine Natriumaufnahme $>5 \mathrm{~g} / \mathrm{Tag}$ ist mit einem Blutdruckanstieg und dem vermehrten Auftreten einer systolischen Hypertonie im Alter assoziiert [169]. Umgekehrt wurde in randomisierten Studien der Einfluss von moderater Natriumreduktion in der Diät bei hypertensiven und normotensiven Menschen untersucht. In einer Meta-Analyse fand sich, dass eine Reduktion der Zufuhr von Natrium um 1,75g/Tag (entsprechend 4,4g Kochsalz/Tag) mit einer Blutdrucksenkung von 4,2/2,2 mm Hg einherging [170], wobei der Effekt bei Hypertoniker/innen stärker war $(-5,4 / 2,8 \mathrm{~mm} \mathrm{Hg})$. Die blutdrucksenkende Wirkung von Natrium-Restriktion ist bei älteren Patient/innen sowie bei solchen mit metabolischem Syndrom, Diabetes oder chronischer Niereninsuffizienz stärker ausgeprägt [171]. Natriumrestriktion bewirkt auch unter medikamentöser Therapie eine effektive Blutdrucksenkung [172]. Besonders der Effekt der Hemmer des Renin-Angiotensin-Aldosteron-Systems (RAAS-Hemmer) wird durch Natrium-Restriktion verstärkt [173]. Bei resistenter Hypertonie kommt der Natrium-Restriktion besondere Bedeutung zu [174]. Die übliche Natriumzufuhr beträgt 3,5-5,5g/Tag (entsprechend 9-12g Kochsalz/Tag). Eine Einschränkung auf 2 g Natrium (5 $\mathrm{g}$ Kochsalz) pro Tag ist zu empfehlen.

\section{Umfassendere Diäten}

Dietary Approaches to Stop Hypertension(DASH-)Diät [175] Diese Diät enthält viel Obst, Gemüse, fettarme Milchprodukte, Vollkornprodukte, Geflügel, Fisch und Nüsse - aber wenig Zucker und rotes Fleisch. Dadurch enthält sie viel Kalium, Magnesium, Kalzium, Eiweiß und Fasern - aber wenig Fett, v. a. gesättigte Fettsäuren und Cholesterin. In einer Studie wurde die DASH Diät mit einer typischen amerikanischen Ernährung verglichen. Die DASH Diät reduzierte den Blutdruck um 11/3 mm Hg - obwohl Natriumaufnahme und Kaloriengehalt ident waren. Kombiniert man die DASH-Diät mit Natriumrestriktion, addieren sich die Effekte. Die mediterrane Diät enthält reichlich Gemüse, Obst, Hülsenfrüchte, Olivenöl, Nüsse, Fisch, aber wenig Fleisch [176]. Sie senkt den Blutdruck [177] und verbessert das Glukose- und Lipidprofil. In einer randomisierten Studie bei Hochrisikopatient/innen traten nach 5 Jahren mediterraner Diät um 29\% weniger kardiovaskuläre Ereignisse und um $39 \%$ weniger Schlaganfälle auf [176].

\section{Andere Empfehlungen zur Ernährung}

Der Genuss von Kaffee führt bei vielen Patient/innen zu einer akuten Blutdrucksteigerung [178]. Langfristig betrachtet, ist moderater Kaffeekonsum (3-5 Tassen pro Tag) aber mit einer Verminderung kardiovaskulärer Ereignisse (Myokardinfarkte, Schlaganfälle) assoziiert [179]. Dies gilt nicht für koffeinfreien Kaffee, der keinen Effekt auf kardiovaskuläre Ereignisse hat. Der Genuss von grünem oder schwarzem Tee kann den BD senken [180, 181]. Eine Kalium-reiche Ernährung (3,5-5 g/Tag; bevorzugt aus Obst, Gemüse, Milchprodukten, Soja, Fisch) kann ebenfalls den BD senken [182] und das Schlaganfall-Risiko vermindern [183]. Sie ist aber bei Niereninsuffizienz oder Einnahme von Kalium-sparenden Medikamenten kontraindiziert.

\section{Reduzierter Alkoholkonsum}

Frauen, die 2 oder mehr alkoholische Getränke pro Tag bzw. Männer, die 3 oder mehr alkoholische Getränke pro Tag konsumieren, weisen signifikant häufiger hypertensive Blutdruckwerte auf als Menschen, die keinen Alkohol trinken [184, 185]. Dieser Effekt ist dosisabhängig und besonders ausgeprägt, wenn die Alkoholzufuhr 5 Drinks pro Tag übersteigt. Reduzieren Menschen, die exzessiv viel Alkohol trinken, ihren Alkohol-Konsum, können sie dadurch ihren BD senken. Moderater Alkoholkonsum (1 Standarddrink pro Tag für Frauen und 1-2 Standarddrinks für Männer) hat aber kaum Effekte auf den BD und ist sogar mit einer milden Abnahme des kardiovaskulären Risikos assoziiert. 1 Standard-Drink ist definiert als $125 \mathrm{ml}$ Wein oder $250 \mathrm{ml}$ Bier.

\section{Gewichtsreduktion}

Gewichtsnormalisierung bei übergewichtigen Individuen kann eine signifikante Blutdruckreduktion bewirken - diese tritt unabhängig von körperlichem Training oder Natriumrestriktion auf [186]. In einer MetaAnalyse war eine Gewichtsreduktion von 5,1 kg mit einer Blutdrucksenkung von 4,4/3,6 mm Hg verbunden [187]. Eine zusätzliche Natriumrestriktion potenziert den Effekt [188]. Sowohl Untergewicht als auch Übergewicht sind mit einem erhöhten Mortalitätsrisiko assoziiert. Der ideale Body-Mass-Index beträgt $22 \mathrm{~kg} / \mathrm{m}^{2}$ für das Alter zwischen 35 und 49 Jahre, $23 \mathrm{~kg} / \mathrm{m}^{2}$ für das Alter zwischen 50 und 69 Jahre, und $24 \mathrm{~kg} / \mathrm{m}^{2}$ für das Alter zwischen 70 und 89 Jahre [189]. Zum Erzielen und Aufrechterhalten einer Gewichtsreduktion sind multidisziplinäre Programme hilfreich, die neben Ernährungsvorschlägen auch Bewegungstherapie und Motivationstechniken umfassen. Anti-AdipositasMedikamente und insbesonders bariatrische Chirurgie können zu einer beträchtlichen Gewichtsreduktion und einer Verbesserung zahlreicher kardiovaskulärer Risikofaktoren führen [190]. 
Tab. 21 Lebensstilmassnahmen bei Hypertonie

\begin{tabular}{|c|c|c|}
\hline Intervention & Empfehlung/Ziel & Effekt auf Blutdruck \\
\hline Kochsalzrestriktion & $\begin{array}{l}5 \mathrm{~g} \text { Kochsalz/Tag } \\
2 \mathrm{~g} \mathrm{Natrium} / \mathrm{Tag}\end{array}$ & $\begin{array}{l}\text { Kochsalzreduktion um 4,4 g/Tag: } \\
\qquad \rightarrow-4,2 / 2,2 \mathrm{~mm} \mathrm{Hg}\end{array}$ \\
\hline Gewichtsreduktion & $\begin{array}{l}\text { BMI 20-25 kg/m² } \\
\text { Bauchumfang: } \\
<94 \mathrm{~cm}(\mathrm{~m}) /<80 \mathrm{~cm} \text { (f) }\end{array}$ & $\begin{array}{l}\text { Gewichtsreduktion } 5,1 \mathrm{~kg}: \\
\rightarrow-4,4 / 3,6 \mathrm{~mm} \mathrm{Hg}\end{array}$ \\
\hline Ernährung & $\begin{array}{l}\text { Reich an Gemüse, Obst, Fisch, } \\
\text { Nüsse, Olivenöl; arm an Fleisch }\end{array}$ & $\begin{array}{l}\text { DASH Diät: } \\
\rightarrow-11 / 3 \mathrm{~mm} \mathrm{Hg}\end{array}$ \\
\hline Kalium & 3,5-5g/Tag (aus 0bst, Gemüse) & $\rightarrow-4-5 \mathrm{~mm} \mathrm{Hg}$ systolisch \\
\hline Alkohol & $\begin{array}{l}<14 \text { Drinks/Woche }(\mathrm{m})^{\mathrm{a}} \\
<8 \text { Drinks/Woche }(\mathrm{f})^{\mathrm{a}} \\
\text { Alkoholexzesse vermeiden }\end{array}$ & $\begin{array}{l}\text { Reduktion von starkem Alkoholkonsum auf empfohlene Menge: } \\
\qquad \rightarrow-4 \mathrm{~mm} \mathrm{Hg} \text { systolisch }\end{array}$ \\
\hline Körperliche Aktivität & Mind. $30 \mathrm{~min}$ an 5-7 Tagen/Woche & $\begin{array}{l}\text { Regelm. aerobes Ausdauertraining bei Hypertonie: } \\
\qquad \rightarrow-8,3 / 5,2 \mathrm{~mm} \mathrm{Hg}\end{array}$ \\
\hline Nikotin & Konsum beenden & - \\
\hline
\end{tabular}

\section{Regelmässige körperliche Aktivität}

Körperliche Aktivität/Sport führt zu einem kurzfristigen Anstieg insbesonders des systolischen BDs, gefolgt von einer kurzanhaltenden Blutdrucksenkung. Aerobes Training und möglicherweise auch Widerstandstraining reduzieren den systolischen und diastolischen BD um 4-6/3 mm Hg. Dieser Effekt ist unabhängig von einem Effekt durch Gewichtsabnahme. Die meisten Studien, die den Effekt von Training auf den BD zeigten, untersuchten drei bis viermal pro Woche moderates aerobes Training für ca. 40 min über 12 Wochen [191]. In einer Meta-Analyse [192] führten aerobes Ausdauertraining, dynamisches Krafttraining und isometrisches Krafttraining zu einer Blutdrucksenkung um 3,5/2,5; 1,8/3,2 und 10,9/6,2 mm Hg. Ausdauertraining war bei Hypertoniker/innen besonders effektiv (Blutdrucksenkung 8,3/5,2 mm Hg). Das Ausmaß der Blutdrucksenkung hängt von der Trainingsintensität $\mathrm{ab}$, aber auch regelmäßige körperliche Aktivität mäßiger Intensität ist bei Hypertoniker/ innen mit niedriger Gesamtsterblichkeit assoziiert [193].

\section{Beendigung des Nikotinkonsums}

Zigarettenrauchen führt zu einem akuten Anstieg von BD [194] und Gefäß-Steifigkeit [195]. Beendigung des Nikotinkonsums zeigt aber keinen Effekt auf den Office-BD [196]. Nikotinkonsum ist weltweit hinter erhöhtem BD die zweitwichtigste Ursache für vorzeitige Sterblichkeit [3]. Eine Beendigung des Nikotinabusus ist vermutlich die effektivste Einzelmaßnahme (siehe Tab. 21) in der Primärprävention kardiovaskulärer Erkrankungen, wobei im wesentlichen BD-unabhängige Mechanismen wirksam werden.

\section{Blutdrucksenkende Medikamente}

Die primäre Intention einer Blutdruckbehandlung ist die Verhinderung kardiovaskulärer Ereignisse durch das dauerhafte Erreichen der Blutdruckzielwerte. In großen Meta-Analysen wurde gezeigt, dass die fünf wichtigsten Antihypertensiva-Klassen (AngiotensinConverting-Enzym-Hemmer - ACE-Hemmer, Angiotensin Rezeptor Blocker - ARBs; Kalziumantagonisten, Diuretika, Betablocker) sicher den BD senken und im Vergleich zu Placebo kardiovaskuläre Ereignisse reduzieren [197, 198]. Der wesentliche Effekt dürfte die Blutdrucksenkung per se sein [198]. Diese 5 Medikamenten-Klassen stellen aufgrund ihrer nachgewiesenen Reduktion kardiovaskulärer Ereignisse die antihypertensive Standardmedikation dar. Andere Antihypertensiva (Alphablocker, zentral wirksame Antihypertensiva, Mineralokortikoid-RezeptorAntagonisten) sind wertvolle Ergänzungsmedikamente, wenn die BD-Zielwerte mit der Standardtherapie nicht erreicht werden.

Eine Meta-Analyse von Studien, die verschiedene Antihypertensiva-Klassen miteinander verglich, zeigte, dass die meisten kardiovaskulären Endpunkte bei den Vergleichen gleich häufig auftraten. Im Detail fanden sich zwar einige Unterschiede, deren praktische Bedeutung aber gering ist, da das Ziel der Therapie eine Verhinderung möglichst aller kardiovaskulären Endpunkte (und nicht nur einzelner ausgewählter) ist: Diuretika waren in der Verhinderung von Herzinsuffizienz überlegen; Kalziumantagonisten waren in der Verhinderung von Herzinsuffizienz unterlegen, aber in der Verhinderung von Schlaganfällen überlegen; ACE-Hemmer waren in der Verhinderung von Schlaganfällen unterlegen, aber in der Verhinderung von Herzinfarkten überlegen; ARBs waren in der Verhinderung von Herzinfarkten unterlegen; Betablocker waren in der Verhinderung von Schlaganfällen unterlegen; ACE-Hemmer und ARBs waren in der Verhinderung von Herzinsuffizienz überlegen [199]. Wenn man die Heterogenität der Substanzklassen zusätzlich ins Kalkül zieht, ergibt sich aus diesen Studien keine eindeutige Präferenz für eine der Substanzklassen in der initialen medikamentösen Behandlung. Die Präparate sollten in Hinblick auf 
Tab. 22 Die wichtigsten Nebenwirkungen und Kontraindikationen der grossen Antihypertensiva-Klassen

\begin{tabular}{|c|c|c|}
\hline Substanzklasse & Kontraindiziert bei: & Vorsicht bei: \\
\hline ACE Hemmer & $\begin{array}{c}\text { Schwangerschaft } \\
\text { Hyperkaliämie (Serumkalium }>5,5 \mathrm{mmol} / \mathrm{l}) \\
\text { Bilaterale Nierenarterienstenose }\end{array}$ & Frauen im gebärfähigen Alter \\
\hline ARB & $\begin{array}{c}\text { Schwangerschaft } \\
\text { Hyperkaliämie (Serumkalium }>5,5 \mathrm{mmol} / \mathrm{l}) \\
\text { Bilaterale Nierenarterienstenose }\end{array}$ & Frauen im gebärfähigen Alter \\
\hline Beta-Blocker & $\begin{array}{l}\text { Asthma } \\
\text { Höhergradiger sinoatrialer oder atrioventrikulärer Block } \\
\text { Bradykardie }(\mathrm{HF}<60 / \text { min) }\end{array}$ & $\begin{array}{l}\text { Metabolisches Syndrom } \\
\text { Glukoseintoleranz } \\
\text { Sportler }\end{array}$ \\
\hline Kalziumantagonist (Dihydropyridin-Typ) & - & $\begin{array}{l}\text { Tachyarrhythmie } \\
\text { HFrEF III/IV } \\
\text { Starke Beinödeme }\end{array}$ \\
\hline Kalziumantagonist (Verapamil, Diltiazem) & $\begin{array}{c}\text { Höhergradiger sinoatrialer oder atrioventrikulärer Block } \\
\text { Bradykardie }(\mathrm{HF}<60 / \mathrm{min}) \\
\mathrm{HFrEF}(\mathrm{EF}<40 \%) \\
\text { Kombination mit Betablocker }\end{array}$ & Obstipation \\
\hline Thiazide/thiazid-ähnliche Diuretika & Gicht & $\begin{array}{l}\text { Metabolisches Syndrom } \\
\text { Glukoseintoleranz } \\
\text { Schwangerschaft } \\
\text { Hyperkalziämie } \\
\text { Hypokaliämie }\end{array}$ \\
\hline
\end{tabular}

ihr Nebenwirkungsprofil und unter Berücksichtigung der Begleiterkrankungen gewählt werden (Tab. 22). Eine mögliche Ausnahme stellen Betablocker in der Initialtherapie bei unkomplizierter Hypertonie (ohne spezifische, meist kardiale Betablocker-Indikation) dar: sie senken den zentralen Blutdruck geringer als ACE-Hemmer/ARBs oder Kalziumantagonisten [200] und führen weniger gut als ACE-Hemmer/ARBs und Kalziumantagonisten zu einer Rückbildung Hypertonie-vermittelter Organschäden [100]. Ein weiterer wichtiger Aspekt der Antihypertensiva sind Nebenwirkungen und dadurch Adhärenz und Therapietreue über lange Zeit [201, 202]. Einen Überblick über die wichtigsten Kontraindikationen der großen Antihypertensiva-Klassen gibt Tab. 22.

\section{Renin-Angiotensin-System-Blocker}

ACE-Hemmer und ARBs sind die weltweit am häufigsten verabreichten Antihypertensiva. Beide Substanzklassen senken die Mortalität sowie die Rate an kardiovaskulären Ereignissen [197]. ARBs zeichnet das geringste Nebenwirkungsprofil aller Antihypertensiva aus [202] (es liegt de facto auf Placeboniveau [202]), was die Chance auf eine gute Medikamentenadhärenz vergrößert [203]. Sie stellen wahrscheinlich auch beim angioneurotischen Ödem - einer seltenen $\mathrm{Ne}$ benwirkung der ACE-Hemmer - eine gute Alternative dar [204]. Hier kann nach 6 Wochen Pause eine Umstellung auf einen ARB erfolgen. Ein weiterer Vertreter der Renin-Angiotensin-Blocker sind Renin-Inhibitoren, die allerdings nach derzeitiger Studienlage keine zusätzlichen Vorteile zu ACE-Hemmern oder ARBs bieten.

Eine klare Indikation für ACE-Hemmer oder ARBs besteht bei Albuminurie, da sie die Progression einer diabetischen und nicht-diabetischen chronischen
Nierenerkrankung verzögern [205] sowie das Risiko einer terminalen Niereninsuffizienz verringern [205], weiters nach stattgehabtem Myokardinfarkt sowie bei Herzinsuffizienz mit reduzierter EF (HFrEF), wobei in diesen kardiologischen Indikationen primär ACEHemmer indiziert sind (und nur bei deren Unverträglichkeit ARBs) [206].

ACE-Hemmer und ARBs sind in der Schwangerschaft aufgrund einer erhöhten Fehlbildungsrate kontraindiziert. $\mathrm{Zu}$ beachten ist ein erhöhtes Risiko einer Hyperkaliämie unter ACE-Hemmern und ARBs, sowie das erhöhte Risiko eines akuten Nierenversagens bei beidseitiger Nierenarterienstenose [199]. Hypertensive Patient/innen mit dunkler Hautfarbe zeigen ein geringeres Ansprechen auf ACE-Hemmer und ARBs, ein häufigeres Auftreten von Angioödemen unter ACEHemmer-Therapie und ein besseres Ansprechen auf Diuretika und Kalziumantagonisten im Vergleich zu ACE-Hemmern und ARBs [207-209].

Eine Kombination von ACE-Hemmern und ARBs (bzw. Renin-Inhibitoren) wird nicht empfohlen. Studien haben gezeigt, dass diese Kombination einerseits keinen zusätzlichen Effekt auf kardiovaskuläre Ereignisse, aber eine erhöhten Rate an Niereninsuffizienz bringt, andererseits sogar mit einer erhöhten Rate an kardiovaskulären Ereignissen bei Diabetiker/ innen verbunden ist [210-212].

\section{Kalziumantagonisten}

Kalziumantagonisten sind eine heterogene Substanzgruppe und werden in zwei Gruppen unterteilt: die Dihydropyridine (DHP; Hauptvertreter in den grossen Outcome-Studien war Amlodipin), zu denen die Datenlage sehr gut ist, sowie die Nicht-Dihydropyridine (Verapamil und Diltiazem), zu denen es eine geringere Anzahl an Studien in der Hypertensiologie gibt. 
Zusammengefasst zeigen die Daten eine Überlegenheit der Kalziumantagonisten zur Prävention von Insulten, allerdings eine Unterlegenheit in der Prävention von Herzinsuffizienz [199]. Letztere war in den großen Outcome-Studien aber schwer zu erfassen, und könnte unter anderem durch die typischen, unter Kalziumantagonisten auftretenden Knöchelödeme überschätzt worden sein.

Vorsicht ist bei Nicht-Dihydropyridinen bei bereits bestehender HFrEF und AV-Block $\geq 2$ gegeben.

\section{Diuretika (Thiazide: Hydrochlorothiazid, Bendrofluazid bzw. thiazidähnliche Substanzen: Chlorthalidon, Indapamid)}

Thiaziddiuretika z.B. Hydrochlorothiazid (HCT) sind seit langem im Gebrauch und die Erfahrung mit diesen Substanzen ist groß. In Studien konnte gezeigt werden, dass die thiazidähnliche Substanz Chlortalidon eine potentere blutdrucksenkende Wirkung mit längerer Wirkdauer im Vergleich zu HCT aufweist. Auch eine stärkere Reduktion an kardiovaskulären Ereignissen und Mortalität wurde verzeichnet [213, 214]. Eine aktuelle Metaanalyse von placebokontrollierten Studien zeigt aber keine klare Überlegenheit für Thiazide, Chlorthalidon oder Indapamid in Hinblick auf das kardiovaskuläre Outcome [198] im Vergleich zu anderen Substanzklassen.

In Österreich findet sich ein klarer Überhang an Fixkombinationspräparaten mit HCT in Form von Zwei- und Dreifachkombinationen. Dem gegenüber gibt es derzeit lediglich ein Fixkombinationsprodukt mit Chlorthalidon. Aus der derzeitigen Datenlage ist allerdings zumindest von einer Gleichwertigkeit der beiden Substanzen auszugehen, weshalb Chlortalidon vermehrt in den Behandlungsfokus rücken sollte.

Thiazide und Thiazid-ähnliche Diuretika weisen ein etwas ungünstigeres Nebenwirkungsprofil als andere Antihypertensiva auf, was mit einer häufigeren Rate an Absetzen wegen Nebenwirkungen verbunden ist [201, 202]. Typisch sind Hypokaliämie, erhöhte Insulinresistenz und Neuauftreten eines Diabetes mellitus. Diese Stoffwechseleffekte können durch Kaliumsubstitution [215] oder Hinzugabe eines Kaliumsparers [216] abgeschwächt werden. Thiazide und Thiazid-ähnliche Diuretika sind unter einer GFR von $45 \mathrm{ml} / \mathrm{min}$ weniger effektiv und sollten unter einer GFR von $30 \mathrm{ml} / \mathrm{min}$ durch Schleifendiuretika ersetzt werden. Bei einer normalen Nierenfunktion spielen Schleifendiuretika zur Blutdrucksenkung keine Rolle.

Bezugnehmend auf die Risikowarnung von HCT und dem vermehrten Auftreten von NMSC-nichtmelanozytärem (weißem) Hautkrebs sowie Lippenkrebs ist eine Aufklärung der Patient/innen über das potenziell erhöhte Risiko notwendig. Es sei erwähnt, dass diese Warnung auf die Ergebnisse von zwei skandinavischen pharmakoepidemiologischen Studien [217, 218] gestützt ist, die den Zusammenhang zwischen der Einnahme von HCT und der Entstehung von
NMSC nahelegt, allerdings ohne den Hauttyp und die Sonnenexposition der Betroffenen in der Analyse berücksichtigt zu haben. Aus derzeitiger Studienlage ist von einem relativ geringen Risiko von HCT auszugehen, die Aufklärung der Patient/innen und die Empfehlung zu einem regelmäßigen dermatologischen Screening sollen erfolgen. Der Wechsel auf ein anderes Präparat wird, insbesondere bei erhöhtem individuellem Risiko für NMSC, wie z. B. bei Einnahme von Immunsuppressiva, unter Berücksichtigung einer Nutzen-Risiko-Erhebung empfohlen.

\section{Betablocker}

Betablocker haben ihren Platz in der Bluthochdrucktherapie bei spezifischer, meist kardialer Indikation (wie z.B. nach Herzinfarkt, bei Vorhofflimmern, HFrEF, aber auch in der Schwangerschaft). Bei Patient/innen mit koronarer Herzerkrankung (KHK) und Status post Myokardinfarkt (MCI) zeigt sich in MetaAnalysen ein Vorteil für Betablocker im Vergleich zu anderen Antihypertensiva [219].

Betablocker erhöhen, besonders in Kombination mit Thiaziden, das Risiko für Neuauftreten eines Diabetes mellitus bei prädisponierten Patient/innen (v.a. solchen mit metabolischem Syndrom). Das Nebenwirkungsprofil ist ungünstiger als bei ARBs, was $\mathrm{zu}$ vermehrtem Absetzen führen kann [201]. Erwähnt sei, dass eine periphere arterielle Verschlusserkrankung (pAVK) keine Kontraindikation für die Betablockertherapie darstellt - es gibt keine Evidenz, dass Betablocker eine Claudicatio intermittens verschlechtern [220, 221]. Auch die chronisch-obstruktive Lungenerkrankung (COPD) gilt nicht mehr als Kontraindikation [222].

Betablocker sind allerdings keine homogene Substanzklasse, insbesonders das Nebenwirkungsprofil betreffend. Neuere Betablocker, teils mit vasodilatierender Wirkung (Carvedilol [223], Nebivolol [224]), haben wenig bis keine ungünstigen metabolischen Nebenwirkungen.

\section{Aldosteronantagonisten}

Spironolacton rückt nicht zuletzt durch die Ergebnisse der PATHWAY-2-Studie [225] in den Behandlungsfokus bei resistenter Hypertonie. Es wird empfohlen, dass bei Nichterreichen der BD-Zielwerte unter Standard-Dreifachtherapie und fehlenden Kontraindikationen (wie schwere Niereninsuffizienz und Hyperkaliämie) Spironolacton hinzugefügt werden soll. Eine Kontrolle des Serumkaliums bei Verabreichung von ACE-Hemmern/ARBs in Kombination mit Spironolacton wird insbesondere bei eingeschränkter Nierenfunktion empfohlen. In einer Substudie der PATHWAY-2-Studie zeigte sich Amilorid im blutdrucksenkenden Effekt vergleichbar mit Spironolacton und stellt daher eine Alternative dar [132]. Auch bei dieser Substanz besteht aber ein Hyperkaliämie-Risiko. 
Tab. 23 Medikamentöse antihypertensive Therapie

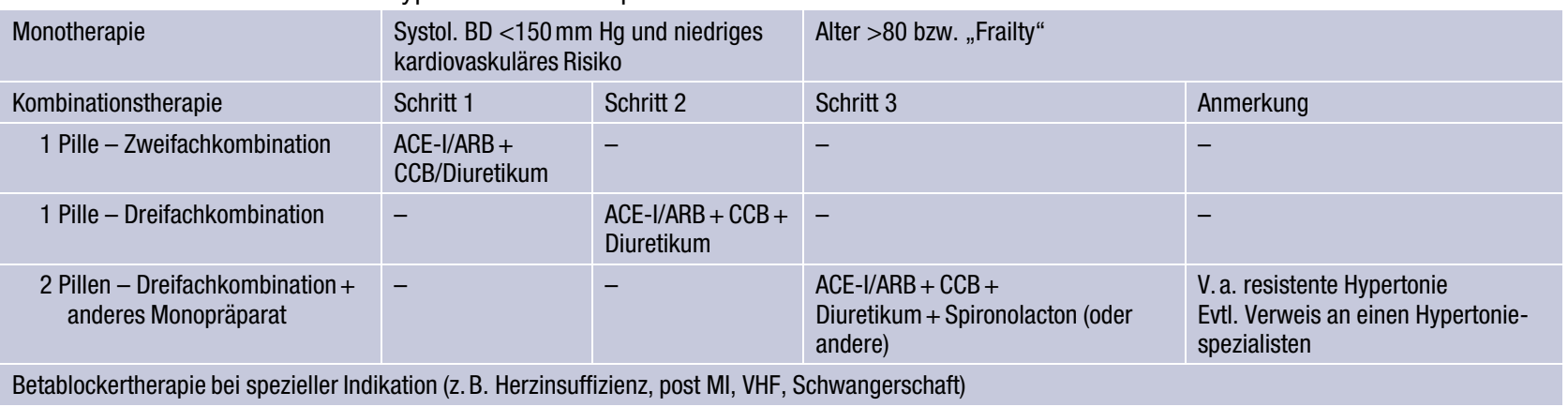

\section{Andere Antihypertensiva}

Alphablocker zeigten sich im Vergleich zu Spironolacton in Hinblick auf die blutdrucksenkende Wirkung in der PATHWAY-2-Studie unterlegen [225], im Vergleich zu Placebo war allerdings eine BD-Senkung nachweisbar. In der ALLHAT-Studie wurde der AlphablockerArm (Doxazosin) wegen des erhöhten Auftretens von Herzinsuffizienz (im Vergleich zu Chlorthalidon) vorzeitig gestoppt [226]. Bei gleichzeitig bestehenden Komorbiditäten wie der benignen Prostatahypertrophie können sie allerdings eine Rolle spielen.

Eine Meta-Analyse zu zentral wirksamen Antihypertensiva, die sehr alte Studien v. a. mit Reserpin und Methyldopa umfasste, zeigte eine Reduktion kardiovaskulärer Ereignisse im Vergleich zu Placebo [198]. Zu den neueren Substanzen (Moxonidin, Rilmenidin) liegen keine kardiovaskulären Endpunktstudien bei Hypertonie vor, die blutdrucksenkende Wirkung ist aber gut belegt.

Zusammenfassend sei festgehalten, dass Alphablocker, zentralwirksame Antihypertensiva und andere Antihypertensiva im Gegensatz zu den 5 Hauptsubstanzen nur als add-on-Therapie bei therapieresistenter Hypertonie eingesetzt werden sollen und in der Standardtherapie keinen Stellenwert mehr haben.

\section{Praktisches Vorgehen}

Wenn das Blutdruckziel mit einer Monotherapie in Standarddosierung nicht erreicht wird, ergeben sich grundsätzlich 2 Möglichkeiten: maximales Aufdosieren der Monotherapie oder Kombination mit einem anderen Antihypertensivum. Beides wurden in einer großen Meta-Analyse (354 randomisierte Studien, 40.000 Patient/innen) miteinander verglichen [227]: Im Durchschnitt senkte eine Standarddosis eines Antihypertensivums einer der 5 Haupt-Klassen (ACEHemmer, ARBs, Betablocker, Kalziumantagonisten, Diuretika) den BD um 9,1/5,5 mm Hg, eine halbe Standard-Dosis um 7,1/4,4 mm Hg. Eine Verdoppelung der Dosis bringt somit nur eine relativ geringen zusätzlichen BD-Senkung. Die Rate an Nebenwirkungen nimmt im Gegensatz dazu aber besonders bei Diuretika, Betablockern und Kalziumantagonisten deutlich zu. Die Kombination mit einem weiteren
Antihypertensivum einer anderen Klasse (Ausnahme: Kombination ACE-Hemmer plus ARB) ist hingegen additiv antihypertensiv wirksam [228], d.h. es kommt zu einer doppelt so starken BD-Senkung.

In den meisten großen Studien hat sich gezeigt, dass für das Erreichen des Blutdruckziels eine Kombinationstherapie erforderlich ist [52].

\section{Initiale Monotherapie oder Kombinationstherapie}

Bislang wurde nur bei deutlich erhöhtem BD eine initiale medikamentöse Kombinationstherapie empfohlen [100]. In mehreren seither publizierten Studien zeigte sich, dass eine initiale Kombinationstherapie eine raschere, verlässlichere und gleich nebenwirkungsarme BD-Senkung und ein rascheres Erreichen der Blutdruckzielwerte ermöglicht [229, 230]. Auch die Rate an Therapieabbrüchen ist reduziert [231]. Eine initiale Kombinationstherapie reduziert auch die „therapeutic inertia“, die unveränderte Fortführung einer bestehenden Therapie trotz insuffizienter Blutdruckkontrolle. Die Monotherapie hat somit nach derzeitiger Datenlage eine untergeordnete Rolle und soll nur bei Patient/innen mit niedrigem Risikoprofil und systolischen Blutdruckwerten $<150 \mathrm{~mm} \mathrm{Hg}$ als Initialtherapie angewendet werden, oder wenn eine langsame BD-Senkung empfehlenswert erscheint, z.B. bei hochbetagten Patient/innen. Die Kombinationstherapie stellt abgesehen von oben genannten Ausnahmen die initiale Standardtherapie dar. $\mathrm{Zu}$ Beginn empfiehlt sich eine 2-fach Kombinationstherapie mit einem ACE-Hemmer oder ARB und einem CCB [232, 233] oder einem Diuretikum [234] (siehe Tab. 23, Abb. 4). Beide Kombinationen wurden in zahlreichen Outcome-Studien getestet.

Mit einer Zweifach-Kombinationstherapie sollten bis zu zwei Drittel aller Patient/innen normotensive Blutdruckwerte erreichen [228]. Wenn erforderlich, folgt als nächster Schritt eine 3-fach Kombination mit den 3 vorher genannten Substanzgruppen, wodurch eine ausreichende Blutdruckkontrolle in bis $\mathrm{zu} 80 \%$ der Patient/innen erreichbar sein sollte [235]. Dies ist deutlich besser, als die derzeitige Situation in Österreich [15] (siehe Abschn. 2 Die Situation in Österreich). Um die Adhärenz zu verbessern [229], empfiehlt sich die Gabe von Fixkombinationpräparaten, die 2 bzw 


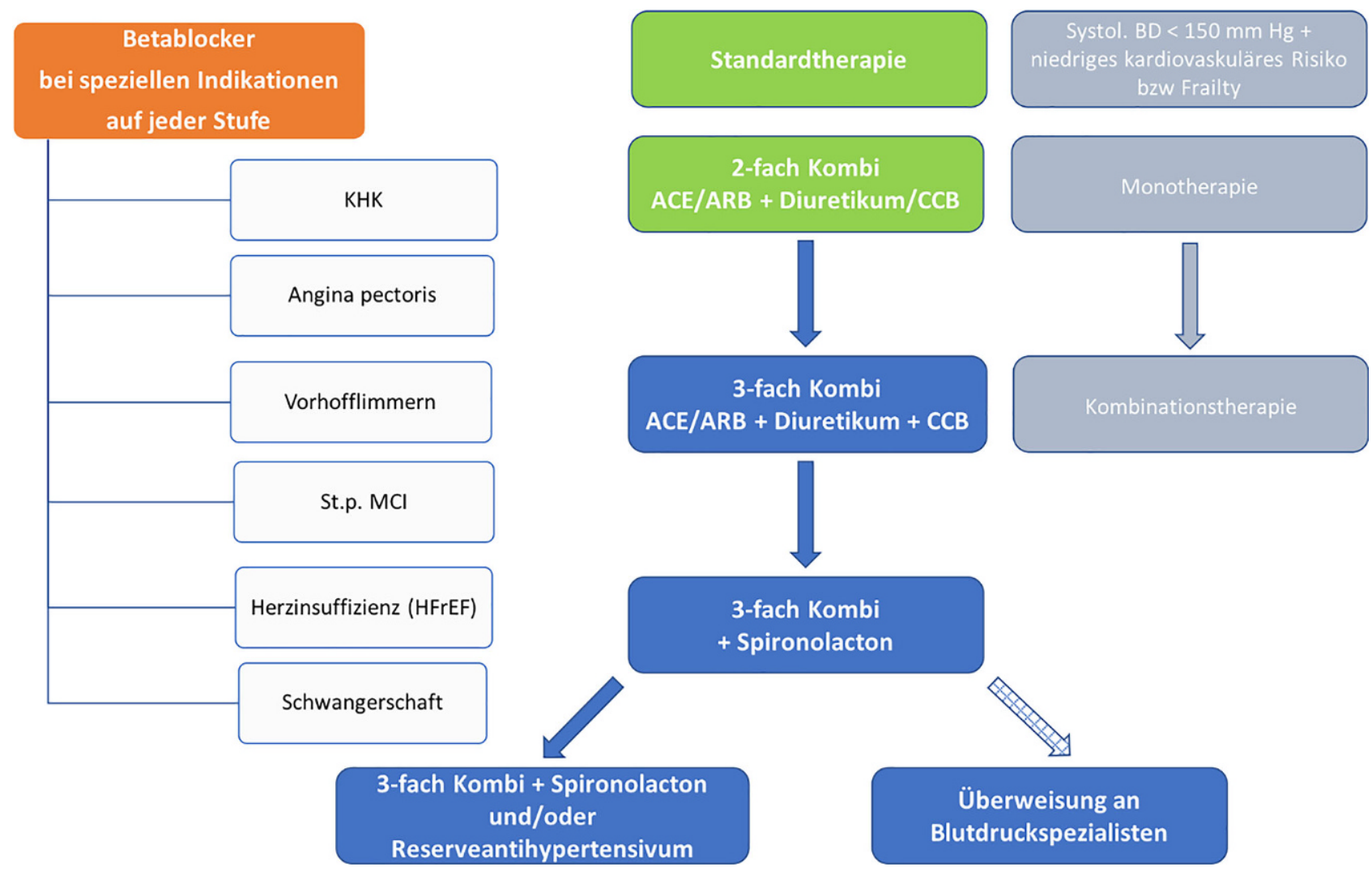

Abb. 4 Medikamentöse antihypertensive Therapie bei Patient/innen mit unkomplizierter Hypertonie. Der Algorithmus ist auch für die meisten Patient/innen mit Hypertonie-vermittelten
Organschäden, zerebrovaskuläre Erkrankungen, Diabetes und pAVK anwendbar. Betablocker werden bei spezifischen Indikationen auf jeder Stufe eingesetzt
3 Substanzen in 1 Tablette vereinen. Leider ist hier derzeit die Auswahl vor allem im Bereich der Diuretika beschränkt.

Ist mit einer 3-fach-Kombination der Zielblutdruck nicht erreichbar, so sollte ein Aldosteronantagonist hinzugefügt werden. Weitere Möglichkeiten sind ein Alphablocker, ein zentral wirksames Antihypertensivum (Clonidin, Alpha-Methyldopa; Moxonidin, Rilmenidin), ein Betablocker, ein Schleifendiuretikum (besonders bei GFR $<45 \mathrm{ml} / \mathrm{min}$ ), oder ein anderes Antihypertensivum (Urapidil, Minoxidil). Per definitionem besteht dann eine therapierefraktäre Hypertonie (siehe Abschn. 23 Resistente Hypertonie).

\section{Fact-Box medikamentöse antihypertensive The- rapie}

- ACE-Hemmer und ARBs, Betablocker, Kalziumantagonisten und Thiazide/Thiazid-ähnliche Diuretika führen zu einer Blutdrucksenkung und reduzieren das Auftreten von kardiovaskulären Ereignissen.

- Als initiale Standardtherapie wird eine 2-fach-Kombination von einem ACE-Hemmer oder ARB mit einem Kalziumantagonisten oder Diuretikum empfohlen optimalerweise in einer Tablette. Je nach Komorbiditäten können alternativ andere Zweifach-Kombinationen aus den 5 Substanzgruppen gewählt werden.
- Die Verdoppelung der Dosis eines Antihypertensivums bringt nur eine relativ geringe zusätzliche Blutdrucksenkung, aber eine Zunahme an Nebenwirkungen. Die Kombination zweier AntihypertensivaKlassen ist additiv wirksam.

- Falls eine Zweifach-Kombinationstherapie nicht ausreicht, soll eine 3-fach-Kombination von einem ACEHemmer oder ARB mit einem Kalziumantagonisten und einem Diuretikum erfolgen - optimalerweise in einer Tablette.

- Als nächster Schritt bei Verfehlen der Blutdruckzielwerte soll der 3-fach-Kombination Spironolacton hinzugefügt werden.

- Eine Monotherapie soll nur noch in speziellen Situationen (z. B. systolischer Blutdruck $<150 \mathrm{~mm} \mathrm{Hg}$ und niedriges kardiovaskuläres Risiko) etabliert werden.

- Eine Kombination von ACE-Hemmern und ARBs ist kontraindiziert.

\section{Blutdruckziele bei unkomplizierter Hypertonie}

Die medikamentöse Behandlung des Bluthochdrucks ist eine der am besten belegten medizinischen Maßnahmen überhaupt: In einer großen Meta-Analyse, die 68 randomisierte Studien und beinahe 250.000 Patient/innen umfasste [11], führte eine standardisierte Senkung des systolisch/diastolischen BDs um 
10/5 mm Hg zu einer Reduktion des relative Risikos für Schlaganfall um $37 \%$, für Myokardinfarkt um $22 \%$, für Herzinsuffizienz um $46 \%$, für kardiovaskuläre Sterblichkeit um $20 \%$, und für Gesamtsterblichkeit um 12\%. Die Zahl der Patient/innen, die über 5 Jahre behandelt werden müssen, um einen Fall von Schlaganfall/Myokardinfarkt/Herzinsuffizienz/ kardiovaskulären Tod zu verhindern, hängt vom Gesamtrisiko ab und betrug im Gesamtkollektiv der Studie 67/118/81 und 134. Aufgrund der relative kurzen Nachbeobachtungszeit in randomisierten Studien von etwa 5 Jahren ist es wahrscheinlich, dass die positiven Effekte der medikamentösen Blutdrucksenkung mit den oben angegebenen Zahlen unterschätzt werden. Studien, die auf genetisch determinierten BDUnterschieden beruhen, die ja während der gesamten Lebenszeit wirksam sind, legen nahe, dass ein um $10 \mathrm{~mm} \mathrm{Hg}$ niedrigerer systolischer $\mathrm{BD}$ das Risiko für Myokardinfarkt um beinahe $50 \%$ verringern könnte [236].

\section{Grundlagen - randomisierte Studien und Meta- Analysen}

In den größten veröffentlichten Beobachtungsstudien [5, 6] steigt das kardiovaskuläre Risiko ab einem systolischen BD von $115 \mathrm{~mm} \mathrm{Hg}$ und ab einem diastolischen BD von $75 \mathrm{~mm} \mathrm{Hg}$ an, ohne dass ein Schwellenwert erkennbar ist. Dennoch können Grenzwerte, ab denen eine medikamentöse Blutdrucksenkung sinnvoll ist, sowie Zielwerte dieser Therapie nur auf Basis randomisierter kontrollierter Studien empfohlen werden, da auch die möglichen Nachteile einer medikamentösen Therapie berücksichtigt werden müssen. Allerdings haben diese Studien auch Limitationen: sie werden meist mit (hoch-)selektierten Patient/ innen durchgeführt, die häufig ein hohes kardiovaskuläres Risiko aufweisen, was die Anwendbarkeit auf die Patient/innen im Alltag oft erschwert; sie haben oft eine Dauer von höchstens 5 Jahren, was mögliche Vorteile einer Langzeittherapie unterschätzt, aber auch mögliche negative Spätfolgen nicht berücksichtigt; sie sind für bestimmte Patient/innengruppen, z. B. junge Patient/innen mit niedrigem Kurzzeitrisiko, nicht verfügbar; und zuletzt wurden bislang alle relevanten Therapie-Studien mit „harten“ kardiovaskulären Endpunkten wie Myokardinfarkt oder Insult ausschließlich auf der Basis des Office-BDs durchgeführt, wodurch sie sich schwer auf die ansonsten ja überlegenen Out-of-Office Messungen umlegen lassen.

In einer großen Meta-Analyse (147 Studien, 464.000 Patient/innen) [219], die neben „echten“ HypertonieStudien auch andere Studien mit Blutdrucksenkern (z. B. Herzinsuffizienz-Studien) umfasste, war der Benefit der medikamentösen BD-Senkung (Reduktion von Myokardinfarkt und Schlaganfall) unabhängig vom Ausgangs-BD und auch bei den niedrigsten erreichten systolischen $(110-119 \mathrm{~mm} \mathrm{Hg})$ und diastolischen (70-74 mm Hg) BD-Werten nachweisbar. Die
Risikoreduktion war abhängig von der BD-Senkung und bei Verwendung von 3 antihypertensiven Medikamenten in halber Standarddosierung etwa doppelt so groß wie bei Verwendung eines Antihypertensivums in Standarddosis. Eine neuere Meta-Analyse, die ebenfalls alle Studien mit BD-Senkern unabhängig von Begleiterkrankungen wie Myokardinfarkt und Herzinsuffizienz einschloss (123 Studien, 613.815 Patient/innen), kam zu ähnlichen Ergebnissen, insbesonders war der Benefit der BD-Senker auch bei niedrigem Ausgangs-BD ( $<130 \mathrm{~mm}$ Hg systolisch) und unabhängig von den Komorbiditäten nachweisbar [197].

Rezent wurde eine Serie von Meta-Analysen veröffentlicht, in denen der Effekt BD-senkender Medikamente bei Hypertonie untersucht wurde [11]. Insgesamt wurden 68 randomisierte Studien (245.885 Patient/innen) eingeschlossen. Eine standardisierte BDSenkung um systolisch/diastolisch 10/5 mm Hg reduzierte das relative Risiko für Insult/Myokardinfarkt/ Herzinsuffizienz/kardiovaskulären Tod/Gesamtsterblichkeit um 36/18/38/16 und $10 \%$, wobei die Number needed to treat über 5 Jahre bei $53 / 133 / 53 / 125 / 111$ lag. Die Risikoreduktion kombinierter vaskulärer Ereignisse war proportional zur BD-Senkung. Basierend auf 14 Studien (behandelte Hypertoniker/innen, ältere Hypertoniker/innen, Patient/innen mit isolierter systolischer Hypertonie, mit und ohne Diabetes, mit renaler Funktionseinschränkung, nach lakunärem Insult) mit 42.354 Teilnehmer/innen, die $\mathrm{zu} 2$ unterschiedlichen BD-Zielen randomisiert wurden, fanden die Autoren, dass eine intensivere BD-Senkung Schlaganfälle, Myokardinfarkte und die kardiovaskuläre Sterblichkeit reduziert [237].

Eine weitere Analyse nach unterschiedlichem BD zu Studienanfang zeigte, dass die Effekte der BDSenkung relativ (prozentuell) gesehen bei jedem Ausgangs-BD gleich sind, die absolute Risikoreduktion aber von der Höhe des Ausgangs-BDs und des kardiovaskulären Risikos abhängen [237, 238], d.h. bei höherem kardiovaskulären Risiko höher sind. Eine Analyse nach erzieltem BD zeigte, dass auch in Studien mit erzieltem systolischen $\mathrm{BD}<130 \mathrm{~mm} \mathrm{Hg}$ und erzieltem diastolischen $\mathrm{BD}<80 \mathrm{~mm} \mathrm{Hg}$ signifikante Risikoreduktionen für Myokardinfarkt, Insult und kardiovaskuläre Mortalität nachweisbar waren. Die absoluten Risikoreduktionen waren aber in den Studien mit höheren erreichten BD-Zielwerten grösser. Mit anderen Worten, auch der niedrigste untersuchte BD-Zielwert (unter $130 \mathrm{~mm} \mathrm{Hg}$ systolisch bzw erreichter systolischer BD 120-129 mm Hg) war vorteilhaft, der Vorteil der BD-Senkung war aber geringer (und die Number needed to treat höher) als der Vorteil der BD-Senkung unter 150 oder unter $140 \mathrm{~mm} \mathrm{Hg}$. Was den diastolischen BD betrifft, war die Senkung unter $80 \mathrm{~mm} \mathrm{Hg}$ (bzw. ein erreichter diastolischer BD von 75-79 mm Hg) vorteilhaft, dies war unabhängig vom Alter [239]. Der Einfluss des systolischen AusgangsBDs in der Primärprävention wurde in einer weite- 
ren Meta-Analyse [240] (51 Studien, 192.795 Patient/ innen) untersucht: die Reduktion von kardiovaskulärer Mortalität, Myokardinfarkt und Schlaganfall war bei einem systolischen Ausgangs-BD $\geq 160 \mathrm{~mm} \mathrm{Hg} \mathrm{am}$ grössten und zwischen 140 und $159 \mathrm{~mm} \mathrm{Hg}$ immer noch signifikant. Bei einem Ausgangs-BD $<140 \mathrm{~mm} \mathrm{Hg}$ fand sich lediglich eine nicht-signifikante Senkung des Insult-Risikos sowie eine signifikante Senkung des Risikos für Herzinsuffizienz. Eine weitere MetaAnalyse bezog nur Studien an unbehandelten Personen mit normalem und hoch-normalem BD ein (120-139/80-89 mm Hg), insgesamt 24 randomisierte Studien und 47.991 Personen [205]. Antihypertensive Behandlung resultierte in dieser Analyse in einer grenzwertig signifikanten Reduktion von Insulten und in einer signifikanten Reduktion der Kombination aus Insult und Myokardinfarkt. Der vorteilhafte Effekt war auf Personen mit hohem und sehr hohem kardiovaskulären Risiko beschränkt.

In einer weiteren Analyse [239] wurden die Effekte der medikamentösen BD-Senkung bei jüngeren und älteren Patient/innen untersucht, wobei $114.009 \mathrm{~Pa}-$ tient/innen jünger und 96.549 Patient/innen älter als 65 Jahre eingeschlossen waren. Ältere Patient/innen wiesen dieselben relativen und größere absolute Risikoreduktionen für kardiovaskuläre Endpunkte als jüngere Patient/innen auf, ebenso ein höheres absolutes Risiko für Nebenwirkungen. Auch Patient/innen über 80 Jahre profitieren signifikant von einer antihypertensiven Therapie [241]. Bei älteren Patient/innen (>65 Jahre) wurde in nur einer Studie bei Hypertonie Grad 1 eine Therapie eingeleitet, hier fand sich ein günstiger Effekt. Ein erzielter systolischer BD von $130-139 \mathrm{~mm} \mathrm{Hg}$ war in beiden Altersgruppen $(<65$ und $>65$ Jahre) vorteilhaft. Ein erzielter systolischer $\mathrm{BD}<130 \mathrm{~mm} \mathrm{Hg}$ war in der Altersgruppe >65 Jahre ebenso vorteilhaft, allerdings gibt es hier nur sehr wenige Studien.

Eine weitere unabhängige Meta-Analyse, die eine intensive mit einer weniger intensiven BD-Senkung verglich, wurde 2016 publiziert [242]. Eingeschlossen wurden 44.989 Patient/innen in 19 randomisierten Studien, der mittlere erreichte Office-BD betrug $133 / 76 \mathrm{~mm} \mathrm{Hg}$ in der intensiver behandelten Gruppe und $140 / 81 \mathrm{~mm} \mathrm{Hg}$ in der Standard-Gruppe. In der intensiver behandelten Gruppe traten signifikant weniger kardiovaskuläre Ereignisse, Myokardinfarkte und Schlaganfälle auf. Der absolute Vorteil war bei Patient/innen mit vaskulären Erkrankungen sowie bei Diabetiker/innen am größten. Schwere Nebenwirkungen waren numerisch, aber nicht statistisch häufiger in der intensiver behandelten Gruppe $(1,2 \%$ versus $0,9 \%$ pro Jahr).

$\mathrm{Zu}$ beachten ist, dass mit einer intensiveren BDSenkung das Risiko von Nebenwirkungen ansteigt, vor allem aber die Rate an Patient/innen, die die antihypertensive Therapie wegen Nebenwirkungen absetzen [243]. Dies kann dann die Vorteile einer bis dahin erzielten BD-Senkung zunichte machen.
Eine singuläre Studie, die für die Diskussion um BD-Zielwerte wichtig ist, soll erwähnt werden: In der SPRINT (Systolic Blood Pressure Intervention Trial) Studie [244] wurden 9361 Patient/innen, älter als 50 Jahre (mittleres Alter 68 Jahre), zu einem systolischen Ziel-BD von 120 versus $140 \mathrm{~mm} \mathrm{Hg}$ randomisiert. Einschlusskriterien waren ein hohes kardiovaskuläres Risiko (Framingham Risk Score $>15 \%$, was das 10-Jahres-Risiko für kardiovaskuläre Ereignisse angibt), vorbestehende kardiovaskuläre Erkrankungen, mäßige Nierenfunktionseinschränkung, oder Alter $\geq 75$ Jahre. Wichtige Ausschlusskriterien waren orthostatische Intoleranz, Diabetes sowie früherer Schlaganfall. Die BD-Messung erfolgte mit der (meist) unbeobachteten automatischen Office-Messung. Nach einer vorzeitigen Beendigung der Studie wegen einer Mortalitätsreduktion in der intensiv behandelten Gruppe nach 3,26 Jahren zeigte sich, dass die intensive Therapie kardiovaskuläre Ereignisse um $25 \%$, Herzinsuffizienz um 38\%, kardiovaskuläre Sterblichkeit um $43 \%$ und Gesamtsterblichkeit um $27 \%$ reduzierte. Ernsthafte Nebenwirkungen traten selten auf, wobei orthostatische Hypotonie, Synkopen (nicht aber Stürze mit Frakturen!), KreatininAnstiege und Elektrolytentgleisungen (Natrium, Kalium) häufiger in der intensiv behandelten Gruppe waren. Der BD unter Therapie lag nach einem Jahr bei $121 / 69 \mathrm{~mm} \mathrm{Hg}$ in der intensiv behandelten Gruppe und bei $136 / 76 \mathrm{~mm} \mathrm{Hg}$ in der Standardgruppe. Aufgrund des Fehlens des Weißkitteleffekts bei der unbeobachteten Office-BD Messung liegen die BDWerte aber tiefer als bei konventioneller Office-Messung und entsprechen eher dem Tages-Durchschnitt bzw. dem 24-Stunden-Durchschnitt des 24-h-Blutdruck-Monitorings [245]. In einer Studie mit mehr als 2000 Patient/innen entsprach ein konventioneller Office-BD von 140/90 mm $\mathrm{Hg}$ einem Wert von $131 / 85 \mathrm{~mm} \mathrm{Hg}$ mit unbeobachteter automatischer Office-BD-Messung [29]. In diesem Zusammenhang ist eine Substudie von SPRINT von Interesse, welche 897 Patient/innen umfasst und in der ein 24h-Blutdruck-Monitoring durchgeführt wurde [31]: in der intensiv behandelten Gruppe lagen der 24-Stunden-Durchschnitt sowie der Tages-Durchschnitt bei $123 / 69 \mathrm{~mm} \mathrm{Hg}$ und 127/72 mm Hg, in der StandardGruppe bei $134 / 74 \mathrm{~mm} \mathrm{Hg}$ und 139/79 mm Hg.

Zusammengefasst, besteht große Evidenz für ein systolisches BD-Ziel zwischen 130 und $139 \mathrm{~mm} \mathrm{Hg}$ (Office-BD) in der allgemeinen, nicht-diabetischen, hypertensiven Population. Eine weitere BD-Senkung auf 120-129 mm Hg kann das kardiovaskuläre Risiko weiter vermindern und soll bei guter Verträglichkeit angestrebt werden, muss aber gegen vermehrte Nebenwirkungen und das Risiko des Absetzens der Therapie wegen Nebenwirkungen abgewogen werden. Die Einleitung einer medikamentösen antihypertensiven Therapie ist ab einem systolischen Office-BD von $140 \mathrm{~mm} \mathrm{Hg}$ immer indiziert, bei hohem kardiovaskulären Risiko, insbesonders bei KHK, kann dies schon 
Tab. 24 Blutdruckzielwerte bei unkomplizierter Hypertonie

\begin{tabular}{l|c|}
\hline $\begin{array}{l}\text { Art der Blutdruckmessung } \\
\text { Office-Blutdruck }\end{array}$ & Zielwert mm Hg \\
\hline Systolischer Blutdruck & $\begin{array}{c}1 . \text { Ziel: } 130 \\
\text { 2. Ziel: } 120-129 \\
\text { (bei guter Verträglichkeit) }\end{array}$ \\
\hline $\begin{array}{l}\text { Diastolischer Blutdruck } \\
\text { Unbeobachtete Automatische Office-Messung }\end{array}$ \\
\hline $\begin{array}{l}\text { Mittelwert dreier Messungen } \\
\text { 24-Stunden-Blutdruck-Monitoring }\end{array}$ \\
\hline 24-Stunden-Mittelwert \\
\hline Tages-Mittelwert \\
\hline Nacht-Mittelwert & $120-125 / 70$ \\
\hline Blutdruck-Selbstmessung & $125-129 / 70-75$ \\
\hline Mittelwert & $115-120 / 65$ \\
\hline
\end{tabular}

ab einem Schwellenwert von $130 \mathrm{~mm} \mathrm{Hg}$ sinnvoll sein. Beim diastolischen BD ist die Situation etwas komplexer: bei Patient/innen jungen und mittleren Alters besteht große Evidenz für ein BD-Ziel unter $90 \mathrm{~mm} \mathrm{Hg}$ und auch ausreichend Evidenz für ein BD-Ziel unter $80 \mathrm{~mm} \mathrm{Hg}$ (Office-BD) (Tab. 24). Bei älteren Patient/innen wird aufgrund einer zunehmenden Steifigkeit der Aorta die isolierte systolische Hypertonie die häufigste Hypertonieform [246]. Bei diesen Patient/innen ist die prognostische Bedeutung des diastolischen BDs dann umgekehrt, d.h. ein niedrigerer diastolischer BD ist mit einer Zunahme an kardiovaskulären Ereignissen assoziiert [7]. Es ist offensichtlich, dass für diese Patient/innen kein diastolisches BD-Ziel angegeben werden kann.

Da bisher keine Therapiestudie primär auf Outof-Office-BD-Werte fokussiert hat (die groß angelegte MASTER-Studie ist im Laufen), muss man bezüglich der Out-of-Office-BD-Zielwerte extrapolieren. Hilfreich dabei ist, dass die Unterschiede zwischen Office-BD und Out-of-Office-BD für die meisten Patient/innen bei niedrigen BD-Werten (etwa $120 / 70 \mathrm{~mm} \mathrm{Hg}$ ) sehr gering werden [247]. Bei behandelten Patient/innen entspricht ein systolischer Office-BD von $130 \mathrm{~mm} \mathrm{Hg}$ etwa einem 24-StundenDurchschnitt von $125 \mathrm{~mm} \mathrm{Hg}$ und einem Durchschnitt in der Selbstmessung von unter $130 \mathrm{~mm} \mathrm{Hg}$. In einer großen Substudie von SPRINT [31] wurde auch 24-Stunden-BD-Monitoring durchgeführt. Die intensiv behandelte Gruppe wies dabei einen 24h-Durchschnitt von 123/69 mm Hg auf, einen Tagesdurchschnitt von 127/72 mm Hg und einen NachtDurchschnitt von 116/63 mm Hg. Die entsprechenden Werte in der Standardgruppe betrugen 134/75 mm Hg (24 Std), 139/79 mm Hg (Tag) und 126/69 mm Hg (Nacht).

\section{Blutdruck und Herzerkrankungen}

\section{Hypertonie und koronare Herzerkrankung}

Ein Anstieg des BDs, beginnend ab $115 \mathrm{~mm}$ Hg systolisch und $70 \mathrm{~mm} \mathrm{Hg}$ diastolisch, ist einer der wichtigsten Risikofaktoren für verschiedenste Manifestationen der KHK (u.a. stabile und instabile Angina pectoris, Myokardinfarkt, plötzlicher Herztod) [5], zumindest bis zum 79. Lebensjahr. Wenn systolischer und diastolischer BD gemeinsam analysiert werden, wie z. B. in der Framingham Studie, ist der diastolische BD bis zum 50. Lebensjahr der beste Prädiktor für eine KHK, zwischen 50. und 59. Lebensjahr sind systolischer und diastolischer BD gleich prädiktiv, und ab den 60 Lebensjahr ist der diastolische BD invers mit dem KHK Risiko assoziiert, sodass der Pulsdruck der beste KHKPrädiktor wird [7].

Physiologisch trägt die Höhe des systolische BDs wesentlich zum myokardialen Sauerstoffverbrauch bei, während die Koronarperfusion unter Ruhebedingungen $\mathrm{zu}$ 85\% während der Diastole erfolgt und somit eng mit dem diastolischen $\mathrm{BD}$ verknüpft ist [248]. Unter starker Belastung verkürzt sich die Diastolenzeit, und $40-50 \%$ des Koronarflusses erfolgt nun in der Systole [249]. Dies kann die subendokardiale Perfusion limitieren, da die intramyokardialen Koronargefässe durch die kardiale Kontraktion in der Systole abgeschnürt werden. Bei jungen Personen mit elastischer Aorta steigt der systolische BD durch das Schlagvolumen nur gering an. Die ebenfalls durch die kardiale Kontraktion erzeugte Pulswelle läuft relativ langsam (Pulswellengeschwindigkeit etwa $5 \mathrm{~m} / \mathrm{s}$ ) in die Peripherie, wird bei Impedanz-Mismatch (Bifurkationen) reflektiert, und läuft ebenso langsam wieder zum Herz zurück. Die reflektierte Druckwelle erreicht im selben Herzzyklus in der Diastole wieder die Aorta ascendens, addiert sich zur antegraden Welle, und erhöht den diastolischen BD (und somit die Koronarperfusion). Wenn die Aorta in höherem Alter steifer wird, steigt die Pulswellengeschwindigkeit, die antegrade und die reflektierte Pulswelle läuft schneller, und die reflektierte Pulswelle erhöht letztlich den spätsystolischen Druck (und somit den Sauerstoffverbrauch des Herzens), während der diastolische BD (und die Koronarperfusion) sinken [250]. In der Tat besteht beim Menschen ein starker inverser $\mathrm{Zu}$ sammenhang zwischen Pulswellengeschwindigkeit und Koronarfluss [251] sowie Koronarreserve [252]. Bei Patient/innen mit manifester KHK besteht ein inverser Zusammenhang zwischen Aortensteifigkeit (Pulswellengeschwindigkeit), Pulswellenreflexionen und der Zeit bis zum Erreichen der Ischämieschwelle in einem Belastungstest [253].

Der Nutzen der antihypertensiven Therapie zur Prävention koronarer Ereignisse ist durch zahlreiche Meta-Analysen ausgezeichnet belegt [11, 219]. Der primäre Mechanismus ist BD-Senkung per se, die 
5 grossen Antihypertensiva-Klassen reduzieren das koronare Risiko gleich gut [219].

Bei etablierter KHK zeigt sich in Meta-Analysen [219] ein Vorteil für Betablocker im Vergleich zu anderen Antihypertensiva nach einem rezenten Myokardinfarkt. Bei KHK-Patient/innen ohne früheren Myokardinfarkt sind alle 5 Antihypertensiva-Klasse gleich gut präventiv wirksam.

BD-Zielwerte und Schwellenwerte zur Einleitung einer antihypertensiven Therapie bei KHK sowie die zugrundeliegenden pathophysiologischen Konzepte wurden rezent in einem Positionspapier der Österreichischen Gesellschaft für Hypertensiologie und der Österreichischen Kardiologischen Gesellschaft ausführlich erörtert [254]. In einer rezenten Meta-Analyse [240] (12 Studien, 77.562 Patient/innen) von Studien mit KHK-Patient/innen fand sich durch BD-Senkung ein signifikant positiver Effekt auf kardiovaskuläre und koronare Ereignisse, Schlaganfall und Herzinsuffizienz, der systolische Ausgangs-BD lag bei $138 \mathrm{~mm} \mathrm{Hg}$. Die Einleitung einer antihypertensiven Therapie bei Patient/innen mit etablierter KHK ist somit schon bei systolischen BD-Werten im hochnormalen Bereich (130-139 mm Hg) zu überlegen. Im Hinblick auf BD-Zielwerte wurde in der CAMELOT Studie [255] (Amlodipin versus Enalapril versus Placebo) gezeigt, dass das Ausmaß der koronaren Atherosklerose, mit intrakoronarem Ultraschall gemessen, bei einem systolischen BD von 120 bis $140 \mathrm{~mm} \mathrm{Hg}$ stabil bleibt, bei niedrigerem $\mathrm{BD}$ abnimmt, und bei höherem $\mathrm{BD} \mathrm{zu}-$ nimmt. Bei allzu niedrigem diastolischen BD ist aber eine Zunahme kardiovaskulärer Ereignisse beschrieben („J-Kurve“) [256]. Dies ist typischerweise durch Patient/innencharakteristika (steife Aorta!) bedingt und muss von einem Therapieeffekt differenziert werden [257]. Dies wurde in der SPRINT Studie gezeigt [258]: Patient/innen in der niedrigsten Quintile des diastolischen BDs (<68 mm Hg) erlitten am häufigsten kardiovaskuläre Ereignisse. Allerdings war auch in dieser Gruppe ein positiver Effekt einer intensiven Senkung des systolischen BDs erkennbar. Im Hinblick auf das hohe kardiovaskuläre Risiko bei etablierter KHK liegt der systolische Ziel-BD $<130 \mathrm{~mm} \mathrm{Hg}$ und der diastolische Ziel-BD <80 mm Hg [259].

Bei Patient/innen mit stabiler KHK sollten Betablocker Teil der medikamentösen antihypertensiven Therapie sein, und zwar aus prognostischen Gründen nach Myokardinfarkt [219] und aus symptomatischen Gründen (Behandlung von Angina pectoris). ACE-Hemmer oder ARBs gehören ebenso $\mathrm{zu}$ den Blutdrucksenkern erster Wahl bei Hochrisikopatient/ innen [260, 261]. Kalziumantagonisten sind gute Alternativen zu Betablockern [100, 262], besonders bei deren Unverträglichkeit [263].

\section{Fact Box Blutdruck und koronare Herzerkran- kung}

- Erhöhter Blutdruck ist ein wesentlicher Risikofaktor für alle Manifestationen der KHK

- In der Primärprävention sind alle Antihypertensiva gleich gut wirksam

- Bei etablierter KHK sind Betablocker (Ausnahme: Kontraindikationen) Teil der antihypertensiven Therapie

- Schwellenwerte (Office-Blutdruck) für die Einleitung einer antihypertensiven Behandlung sind:

- Systolisch 130-139 mm Hg (bei guter Verträglichkeit, sonst $140 \mathrm{~mm} \mathrm{Hg}$ )

- Diastolisch $90 \mathrm{~mm} \mathrm{Hg}$

- Zielwerte (Office-Blutdruck) für die antihypertensive Therapie sind:

- Systolisch 120-129 mm Hg (bei guter Verträglichkeit, sonst $130-139 \mathrm{~mm} \mathrm{Hg}$ )

- Diastolisch 70-79 mm Hg

\section{Hypertonie und Herzinsuffizienz}

Erhöhter BD ist ein wichtiger Risikofaktor für das Auftreten einer Herzinsuffizienz, sowohl mit erhaltener (Heart Failure with preserved Ejection Fraction, HFpEF) als auch mit reduzierter (Heart Failure with reduced Ejection Fraction, HFrEF) Auswurffraktion [263]. Dabei spielen die Höhe des diastolischen, mehr noch des systolischen BDs [264] und des Pulsdrucks [265] eine Rolle. Für die Pathophysiologie sowohl von HFpEF als auch HFrEF sind genauere Parameter der pulsatilen Hämodynamik wie Aortensteifigkeit und Pulswellenreflexionen noch wichtiger [266]. Etwa 3/4 aller Patient/innen, die wegen dekompensierter Herzinsuffizienz hospitalisiert werden, haben eine Hypertonie-Anamnese [267]. Viele Patient/innen mit HFpEF haben einen erhöhten BD-Anstieg unter körperlicher Belastung [268]. Das hypertensive Lungenödem ist eine typische Manifestation von HFpEF. Eine wichtige Vorstufe (und auch Co-Morbidität) bei HFpEF ist eine Linksherzhypertrophie (LVH). Das Ausmaß der LHV-Regression, welche prognostisch günstig ist [269], ist unter ACE-Hemmern, ARB's und Kalziumantagonisten am größten [270], weiters spielt eine BDSenkung auf systolisch 120-130 mm Hg eine wichtige Rolle [271]. Ein Myokardinfarkt ist oft ein wesentlicher Zwischenschritt von der Hypertonie zur HFrEF [272]. Medikamentöse antihypertensive Behandlung konnte das Risiko für das Auftreten einer Herzinsuffizienz in randomisierten Studien mit Patient/innen mittleren Alters beinahe halbieren [11], bei älteren Patient/ innen nach Myokardinfarkt und isolierter systolischer Hypertonie sogar um $80 \%$ reduzieren [273]. Sobald sich eine HFrEF entwickelt hat, normalisiert sich der vorher erhöhte BD häufig [263]. Bei Patient/innen mit Herzinsuffizienz und unkontrolliertem BD ist eine optimale BD-Kontrolle ein vorrangiges Ziel [263]. Da 
Tab. 25 Evidenz-basierte medikamentöse Behandlung bei HFrEF [206] mit Fokus auf die blutdrucksenkende Wirkung

\begin{tabular}{l|l|l|}
\hline & \multicolumn{1}{c}{$\begin{array}{l}\text { Dosis am Beginn } \\
(\mathrm{mg})\end{array}$} & Zieldosis (mg) \\
\hline ACE Hemmer & $3 \times 6,25$ & $3 \times 50$ \\
\hline Captopril & $2 \times 2,5$ & $2 \times 20$ \\
\hline Enalapril & $1 \times 2,5-5$ & $1 \times 20-35$ \\
\hline Lisinopril & $1 \times 2,5$ & $1 \times 10$ \\
\hline Ramipril & $1 \times 0,5$ & $1 \times 4$ \\
\hline Trandolapril & $1 \times 1,25$ & $1 \times 10$ \\
\hline Betablocker & $2 \times 3,125$ & $2 \times 25$ \\
\hline Bisoprolol & $1 \times 12,5-25$ & $1 \times 200$ \\
\hline Carvedilol & $1 \times 1,25$ & $1 \times 10$ \\
\hline Metoprolol succinate (CR/XL) & \\
\hline Nebivolol & $1 \times 4-8$ & $1 \times 32$ \\
\hline Angiotensin Rezeptor Antagonist & $2 \times 160$ \\
\hline Candesartan & $2 \times 40$ & $1 \times 150$ \\
\hline Valsartan & $1 \times 50$ & \\
\hline Losartan & $1 \times 50$ \\
\hline Mineralocorticoid Rezeptor Antagonist & $1 \times 25$ & $1 \times 50$ \\
\hline Eplerenon & $1 \times 25$ & $2 \times 97 / 103 \mathrm{mg}$ \\
\hline Spironolacton & $2 \times 49 / 51$ mg & \\
\hline Angiotensin-Rezeptor Antagonist/Neprilyisn-Inhibitor (ARNI) & \\
\hline Sacubitril/Nalsartan & 25 & \\
\hline
\end{tabular}

der BD besonders bei HFrEF häufig niedrig ist, richtet sich die pharmakologische Therapie bei HFrEF (neurohumorale Blockade mit ACE-Hemmern/ARB's/ ARNI (Sacubitril-Valsartan), Betablockern, Aldosteron-Antagonisten) primär nicht nach speziellen BDZielen, sondern man versucht, die in den großen randomisierten HFrEF-Studien etablierten Zieldosierungen zu erreichen [206, 274]. In diesen Studien hatte sich ja eine klinische Überlegenheit für höhere versus niedrigere Dosierungen von ACE-Hemmern [275] und Betablockern [276] gezeigt. Der klinische Nutzen der Behandlung von HFrEF mit ACE-Hemmern/ARBs und Betablockern hängt nicht vom systolischen BD am Beginn der Therapie oder seiner Änderung unter Therapie ab [277, 278]. Evidenz-basierte medikamentöse Therapie bei HFrEF mit Fokus auf BD-senkende Medikamentengruppen zeigt Tab. 25. Sollten trotz optimaler Dosierung der Standard-HFrEF-Therapie weiterhin erhöhte BD-Werte bestehen, können die Dihydropyridin-Kalziumantagonisten Amlodipin [279] und Felodipin [280] hinzukombiniert werden, da ihre Anwendung bei HFrEF sicher ist. Im Gegensatz dazu sollten die Nicht-Dihydropyridine Verapamil und Diltiazem, das zentral wirksame Moxonidin, der Vasodilatator Minoxidil, und Alphablocker wie Doxazosin bei HFrEF nicht verwendet werden [264]. Schleifendiuretika, die zur Therapie einer Volumsüberladung bei HFrEF und HFpEF notwendig sein können, haben eine geringere BD-senkende Wirkung als ThiazidDiuretika [263, 264]. Weder bei HFrEF noch bei HFpEF gibt es BD-Zielwerte, die durch randomisierte Studien begründet sind.

\section{Fact Box Blutdruck und Herzinsuffizienz}

- Hypertonie ist ein wesentlicher Risikofaktor für HFrEF und HFpEF

- Reduktion des Auftretens von Herzinsuffizienz ist einer der stärksten präventiven Effekte einer antihypertensiven Behandlung

- Bei etablierter HFrEF fällt der BD häufig auf normale oder niedrige Werte ab

- Bei HFrEF ist das primäre Ziel die Etablierung einer optimal dosierten Standardtherapie mit ACE-Hemmern/ARB's/ARNI, Betablockern und AldosteronAntagonisten, unabhängig vom $B D$

- Bei weiterhin erhöhtem BD trotz optimaler Standardtherapie bei HFrEF können Dihydropyridin-Kalziumantagonisten (Amlodopin, Felodipin) verschrieben werden

- Bei Linksherzhypertrophie, einer bedeutsamen Vorstufe bzw. Comorbidität bei HFpEF, sind zum Erreichen der prognostisch wichtigen Regression ACE-Hemmer/ARBs oder Kalziumantagonisten sowie ein systolischer BD-Zielwert von 120-130 mm Hg sinnvoll

\section{Hypertonie und Arrhythmien}

Das Auftreten von Arrhythmien, insbesondere von Vorhofflimmern, kann als Manifestation einer hypertensiven Herzerkrankung angesehen werden, insbesondere beim Vorliegen einer Linksherzhypertrophie [281]. Pathophysiologisch spielen erhöhte linksventrikuläre Füllungsdrucke, verminderte diastolische Funktion, erhöhte linksatriale Drucke mit Vergrößerung und Fibrose des linken Vorhofs und dadurch bedingte elektrische Alterationen (Abnahme der intra- und interatrialen Leitungsgeschwindigkeit) eine Rolle. Auf der anderen Seite ist Hypertonie die häufigste Komorbidität bei Vorhofflimmern [281]. Das Auftreten von Vorhofflimmern ist bei Patient/innen mit Hypertonie mit einem 2,5-fachen Anstieg der Gesamtsterblichkeit verbunden [282]. Eine optimale BD-Kontrolle, dh. 120-130/60-69 mm Hg, war in Observationsstudien mit dem geringsten Auftreten von Vorhofflimmern assoziiert [283]. Hypertonie ist ein Risikofaktor für die Progression von paroxysmalem zu permanentem Vorhofflimmern und für das Auftreten eines Schlaganfalls bei Vorhofflimmern. Eine antihypertensive Behandlung kann das Risiko von Vorhofflimmern reduzieren [284], wobei ACE-Hemmer/ARBs möglicherweise bei Linkshypertrophie oder linksventrikulärer Dysfunktion besonders wirksam sind. Das Vorliegen einer Hypertonie (definiert als Ruhe-BD $>140 / 90 \mathrm{~mm}$ Hg bei zumindest 2 Gelegenheiten oder laufende antihypertensive Medikamente) ist Bestandteil des $\mathrm{CHA}_{2} \mathrm{DS}_{2}$-VASc-Scores, der das thromboembolische Risiko bei Vorhofflimmern abschätzt und 
bei der Entscheidung zur oralen Antikoagulation hilft [285]. Hypertonie erhöht auch das Blutungsrisiko unter oraler Antikoagulation [286], weshalb unkontrollierte Hypertonie (systolischer BD >160 mm Hg) Bestandteil des HAS-BLED-Scores (zur Abschätzung des Blutungsrisikos unter oraler Antikoagulation) ist. Unkontrollierte Hypertonie ( $\geq 170-180 \mathrm{~mm} \mathrm{Hg}$ systolisch und/oder $\geq 100 \mathrm{~mm} \mathrm{Hg}$ diastolisch) war ein Ausschlußgrund in den 4 großen NOAK-Studien [281]. Das Vorliegen einer Hypertonie alleine ergibt schon einen Punkt beim $\mathrm{CHA}_{2} \mathrm{DS}_{2}$-VASc-Score und somit eine „kann“-Indikation zur Antikoagulation, ein weiterer Risikofaktor führt zu einer „soll“ Indikation für eine orale Antikoagulation. Der Vorteil der NOAKs im Vergleich zu den Vitamin K Antagonisten war in den großen Vergleichsstudien auch bei Vorliegen von Hypertonie konsistent [281], wobei erhöhter BD in diesen Studien ein Risikofaktor für kardioembolische Ereignisse war [287]. Bei Patient/innen mit (insbesondere ausgeprägter) Linksherzhypertrophie sollten Sotalol, Flecainid oder Propafenon nicht verwendet werden [281].

Linksventrikuläre Hypertrophie ist auch der wichtigste Prädiktor für das Auftreten supraventrikulärer Tachykardien [281]. Zur Frequenzkontrolle bei Vorhofflimmern und anderen supraventrikulären Tachykardien kommen Betablocker und Nicht-DihydropyridinKalziumantagonisten in Betracht [281].

Bluthochdruck ist, besonders wenn schon eine linksventrikuläre Hypertrophie besteht, mit auch komplexen Extrasystolen und anhaltenden ventrikulären Arrhythmien [288] assoziiert. Erhöhter BD und darüber hinaus eine Zunahme der linksventrikulären Masse sind Risikofaktoren für den plötzlichen Herztod [289]. Als Mechanismen werden u.a. eine Verlängerung der Repolarisation (QT-Intervall), sowie eine Ischämie durch Mismatch zwischen Sauerstoff-Angebot und -Verbrauch bei verminderter Koronarreserve diskutiert [281]. Effektive BD-Kontrolle und besonders Regression einer Linkshypertrophie kann das Arrhythmierisiko und das Risiko des plötzlichen Herztodes senken [290]. Möglicherweise sind ACE-Hemmer/ARBs bei der Arrhythmie-Reduktion besonders effektiv [261]. Im Gegensatz dazu wurde ein Zusammenhang zwischen einer Behandlung mit Thiaziden und einem erhöhten Arrhythmierisiko beschrieben [291], als Mechanismus wird v.a. eine Thiazid-vermittelte Hypokaliämie angenommen.

\section{Fact Box Blutdruck und Arrhythmien}

- Hypertonie ist ein wesentlicher Risikofaktor für supraventrikuläre Arrhythmien, besonders Vorhofflimmern

- Bei Vorhofflimmern erhöht Bluthochdruck sowohl das Thromboembolie-Risiko als auch das Blutungsrisiko
- Hypertonie ist ein wesentlicher Risikofaktor für ventrikuläre Arrhythmien, einschließlich des plötzlichen Herztodes

- Das Vorliegen einer Linksherzhypertrophie erhöht das Arrhythmierisiko erheblich

- Vorrangiges Therapieziel ist eine Normalisierung des Blutdrucks

- Bei Vorhofflimmern sind die Vorteile der NOAKs gegenüber den Vitamin K Antagonisten auch bei Hypertonie belegt

- Das Auftreten einer Hypokaliämie unter antihypertensiver Behandlung mit Thiaziden kann das Arrhythmierisiko erhöhen

- Klasse IC Antiarrhythmika oder Sotalol sind bei erheblicher Linksherzhypertrophie kontraindiziert

\section{Hypertonie und Herzklappenerkrankungen}

Hypertonie ist ein Risikofaktor für die Entwicklung einer hochgradigen Aortenstenose auf Basis einer Aortensklerose oder bikuspiden Klappe und für die Progression einer asymptomatischen Aortenstenose [116]. Insbesondere das Renin-Angiotensin-Aldosteron-System ist bei Aortenstenose aktiviert und scheint mit der Klappenverkalkung in Zusammenhang zu stehen [292]. Bei gleichzeitigem Vorliegen von Aortenstenose und Hypertonie tragen beide zur erhöhten Druckbelastung des linken Ventrikels bei [293]. Bei Patient/innen mit mäßiggradiger Aortenstenose ist das Vorliegen einer Hypertonie mit dem häufigeren Vorliegen einer konzentrischen Linksherzhypertrophie und mit einer erhöhten Gesamtsterblichkeit assoziiert [294]. Die frühere Meinung, dass Antihypertensiva im Allgemeinen und Vasodilatatoren im Besonderen bei hochgradiger Aortenstenose kontraindiziert wären, ist nicht mehr Evidenz-basiert [293]. Die Sicherheit und Verträglichkeit einer Therapie mit Antihypertensiva wurde bei mäßiggradiger [295] und bei schwerer symptomatischer Aortenstenose [296] belegt, wobei bei linksventrikulärer Dysfunktion und initial niedrigem BD Hypotonie auftreten kann. Hemmung des Renin-Angiotensin-Aldosteron-Systems hat einen positiven Effekt auf linksventrikuläre Fibrose, BD-Kontrolle, Dyspnoe und Leistungsfähigkeit [296]. Auch Betablocker werden gut toleriert und verbessern die Symptomatik, weil die Systolendauer verlängert wird [292]. Weiters können sie in speziellen Indikationen (z.B. nach Myokardinfarkt, Arrhythmien) verabreicht werden, ebenso Diuretika bei Volumsüberlastung.

Bei hämodynamisch bedeutsamer Aorteninsuffizienz sollten Antihypertensiva, die die Herzfrequenz senken, eher vermieden werden, da dadurch die Diastolendauer verlängert und das Regurgitationsvolumen erhöht wird. Ein eindeutiger klinischer Vorteil für die Gabe von Vasodilatatoren (Nifedipin, Enalapril) ist bei Aorteninsuffizienz nicht belegt [297]. 


\section{Fact Box Blutdruck und Herzklappenerkrankun-} gen

- Hypertonie ist ein Risikofaktor für die Entwicklung und Progression einer Aortenstenose

- Hypertonie ist bei Aortenstenose ein zweiter Faktor, der die linksventrikuläre Nachlast erhöht

- Hypertonie bei Aortenstenose ist mit ausgeprägterer Linksherzhypertrophie, und einer erhöhten Gesamtsterblichkeit assoziiert

- Eine antihypertensive Behandlung wird auch bei Aortenstenose empfohlen, wobei die Therapie mit niedrigen Dosierungen begonnen und langsam gesteigert werden sollte

- Dabei sind ACE-Hemmer/ARBs möglicherweise zu bevorzugen, Betablocker und Diuretika haben spezielle Indikationen

- Bei Aorteninsuffizienz sollten Antihypertensiva verwendet werden, die die Herzfrequenz nicht senken.

\section{Blutdruck und vaskuläre Erkrankungen}

\section{Carotis-Atherosklerose}

Das Vorhandensein und das Ausmaß einer Hypertonie sind eng mit dem Vorhandensein und dem Schweregrad der Carotis-Atherosklerose assoziiert [298]. Die Evidenz für einen Effekt antihypertensiver Medikamente auf Carotisplaques oder Intima-Media-Dicke (IMT) ist rar gesät. Blutdrucksenkung scheint jedoch die IMT zu reduzieren, besonders Kalziumantagonisten scheinen hier etwas günstiger zu sein als Betablocker, ACE-Hemmer oder Diuretika [299, 300]. Die Relevanz dieser ohnehin nicht überaus robusten Daten ist für die Praxis nicht groß, da häufig Kombinationstherapien eingesetzt werden müssen, und die Korrelation zwischen dem Auftreten eines zerebrovaskulären Ereignisses und der IMT Regression gering ist [301]. Patient/innen mit Carotisplaques haben ein hohes Risiko für atheroembolische und kardiovaskuläre Ereignisse. Daher sollte über die Blutdruckregulation hinaus eine Therapie mit Statinen und Plättchenhemmern bei diesen Patient/innen etabliert werden. Vorsicht geboten ist bei der bilateralen hochgradigen Carotisstenose. Obwohl es hier keine Studiendaten gibt, empfiehlt sich ein pragmatisches Vorgehen. Eine Blutdrucksenkung soll in diesem Fall vorsichtig und moderat und jedenfalls zu Beginn mit Monotherapie erfolgen [52].

\section{Periphere arterielle Verschlusskrankheit}

Hypertonie ist ein starker Risikofaktor für pAVK. Eine Zunahme des systolischen BDs um 20 mm Hg erhöht das Risiko für pAVK um $63 \%$ [302], wobei diese Zunahme des Risikos bei jüngeren Patient/innen deutlich stärker ist. Patient/innen mit manifester pAVK weisen ein besonders hohes kardiovaskuläres Risiko auf, da die Erkrankung üblicherweise mit einer schweren generalisierten Atherosklerose verknüpft ist [67, 302]. Das Erreichen der Blutdruckzielwerte stellt einen wesentlichen Teil der Behandlung dar. Alle großen Antihypertensiva-Klassen wurden erfolgreich in Studien mit einem bedeutenden Anteil an pAVK-Patient/ innen getestet [303]. Im Gegensatz zur landläufigen Meinung gibt es keine Evidenz, dass Betablocker eine Claudicatio intermittens verschlechtern [220, 221]. Daher stellt die pAVK keine Kontraindikation für die Verabreichung von Betablockern dar. Bei Vorliegen einer pAVK IV, soll die Blutdrucksenkung jedoch vorsichtig erfolgen, da eine zu rasche Absenkung das klinische Bild verschlechtern kann. Wie bei anderen Patient/innengruppen kann die Blutdruckeinstellung auch bei pAVK-Patient/innen nur Teil einer Strategie zur kardiovaskulären Risikokontrolle sein. Unbedingt $\mathrm{zu}$ achten ist auch auf eine Optimierung der Stoffwechselparameter, die Einhaltung einer Nikotinkarenz, sowie auf eine adäquate Statin- und - bei symptomatischen Patient/innen sowie nach Revaskularisierung [67] - Antiplättchentherapie.

\section{Erhöhte Gefäßsteifigkeit}

Der Verlust der Elastizität der großen Arterien im Alter führt zur Zunahme des systolischen und Abnahme des diastolischen BDs [246]. Alle antihypertensiven Medikamente führen, alleine durch die Blutdruckreduktion, zu einer Reduktion der Gefäßsteifigkeit, da die Wandspannung der arteriellen Gefäßwand abnimmt, dokumentiert durch eine (passive) Abnahme der Pulswellengeschwindigkeit. Möglicherweise gibt es durch die Beeinflussung der Zusammensetzung der extrazellulären Matrix der Gefäßwand einen additiven günstigen Effekt für ACE-Hemmer und ARBs hinsichtlich Reduktion der PWV, der über die reine blutdrucksenkende Wirkung hinaus geht [304-307].

\section{Aortenektasie, -aneurysma und-dissektion}

Arterielle Hypertonie kann mit einer mäßiggradigen Dilatation der Aortenwurzel einhergehen. Geht die Dilatation über die Aortenwurzel hinaus, ist nach anderen Ursachen für die Ektasie zu suchen. Unabhängig von der Ursache sollen alle Patient/innen mit Aortenektasie oder Aortenaneurysma einen Zielblutdruck von 130/80 mm Hg nicht überschreiten [308]. Für die Wahl der Substanzklasse kann keine generelle Empfehlung abgebeben werden, lediglich für Patient/ innen mit Marfan Sydrom scheint es einen Vorteil für den Einsatz von Betablockern, ACE-Hemmern und ARBs zu geben [309-311]. In Observationsstudien ist Hypertonie mit dem Risiko einer akuten Aortendissektion assoziiert [312]. Insbesondere ein erhöhter nächtlicher systolischer $\mathrm{BD}(>124 \mathrm{~mm} \mathrm{Hg})$ ist mit vermehrten aortalen Komplikationen im Langzeitverlauf nach Typ B Aortendissektion assoziiert [313]. Bei Patient/innen mit Typ A und Typ B Aortendissektionen war die Verwendung von Betablockern bei 
allen Patient/innen mit einer erhöhten Überlebensrate verbunden, während bei Typ B Dissektionen die Verwendung von Kalziumantagonisten, jedoch nicht die Verwendung von ACE-Hemmern das Überleben verbesserte [314]. Bei Typ B Dissektion scheint die endovaskuläre Korrektur einer subklinischen renalen Minderperfusion mit einer besseren Blutdruckeinstellung im Langzeitverlauf einherzugehen [313].

\section{Blutdruck und Diabetes mellitus}

\section{Epidemiologie}

Die arterielle Hypertonie ist eine sehr häufige Komorbidität sowohl bei Patient/innen mit Typ 2 als auch bei Patient/innen mit Typ-1-Diabetes mellitus (allerdings erst wenn diese eine Nephropathie entwickelt haben) [315]. Blutdruckwerte $\geq 140 \mathrm{~mm} \mathrm{Hg}$ systolisch und/oder $\geq 90 \mathrm{~mm} \mathrm{Hg}$ diastolisch finden sich in $\mathrm{Ab}$ hängigkeit von Diabetesdauer und Ausmaß der Adipositas in zumindest 50-60\% aller Patient/innen [316]. Fehlende Nachtabsenkung bzw. eine isolierte systolische Hypertonie bei älteren Patient/innen sind bei Diabetes häufige Befunde, ebenso eine maskierte Hypertonie [317]. Patient/innen mit Hypertonie haben ein 2,2-fach erhöhtes Risiko innerhalb von 5 Jahren einen Diabetes zu entwickeln. Sie sollen deshalb auf das Vorliegen einer Glukosestoffwechselstörung untersucht werden. Das Vorliegen eines Diabetes mellitus bei Hypertonie ist mit einem hohen oder sogar sehr hohen kardiovaskulären Risiko verbunden [49]. Bei der Blutdruckmessung sollte besonders bei langer Diabetesdauer auch eine Messung im Stehen durchgeführt werden, um eine Orthostasereaktion zu erkennen.

\section{Hintergrund für Empfehlungen}

Bei Typ 1 Diabetes ist eine nephroprotektive Wirkung einer Blutdrucksenkung mit Kalziumantagonisten und ACE-Hemmern belegt [318]. Da die in den Studien bisher untersuchten Patient/innen meist jünger waren, waren (noch) keine Effekte auf kardiovaskuläre Endpunkte nachweisbar. Bei Typ-2-Diabetes reduziert Blutdrucksenkung das Risiko für Gesamtsterblichkeit, kardiovaskuläre Ereignisse, koronare Herzerkrankung, Schlaganfall, Albuminurie und Retinopathie [319]. Aufgrund des höheren kardiovaskulären Risikoprofils ist der absolute Vorteil der Blutdrucksenkung bei Diabetiker/innen höher als bei Nicht-Diabetiker/innen, und die Number needed to treat zur Verhinderung eines kardiovaskulären Ereignisses ist bei Diabetiker/ innen geringer [320].

Für das Festlegen von Blutdruckschwellen- und -zielwerten kann man bei Diabetiker/innen auf folgende Evidenz zurückgreifen: In einer grossen Meta-Analyse bei Typ-2-Diabetiker/innen (40 Studien, mehr als 100.000 Patient/innen) [319] fand sich ein eindeutiger Vorteil der Blutdrucksenkung bei einem systolischen Ausgangs-BD von mehr als $140 \mathrm{~mm} \mathrm{Hg}$ hinsichtlich Verringerung aller oben angeführten Endpunkte inklusive Herzinsuffizienz. Bei einem systolischen Ausgangs-BD unter $140 \mathrm{~mm} \mathrm{Hg}$ war nur noch die Verringerung von Schlaganfall und Albuminurie signifikant. Eine Analyse nach „on treatment“ systolischem BD zeigte ein ähnliches Bild: ein eindeutiger Vorteil der Blutdrucksenkung bestand bei einem erreichten systolischen $\mathrm{BD}$ von mehr als $130 \mathrm{~mm} \mathrm{Hg}$ hinsichtlich Verringerung aller oben angeführten Endpunkte. Bei einem erreichten systolischen BD unter $130 \mathrm{~mm} \mathrm{Hg}$ war nur noch die Verringerung von Schlaganfall und Albuminurie signifikant. In einer weiteren grossen Meta-Analyse [320] (41 Studien, mehr als 61.000 Patient/innen) wurde dies bestätigt: bei erreichtem systolischen BD-Werten von 130-140 mm Hg sind die Vorteile der Blutdrucksenkung bei Diabetiker/innen hinsichtlich vieler Endpunkte grösser als für Nicht-Diabetiker/innen. Bei erreichtem systolischen BD-Werten unter 130 mm Hg ist bei den Diabetiker/innen nur noch eine signifikante Schlaganfallreduktion nachweisbar (ein gehäuftes Auftreten kardiovaskulärer Endpunkte ist bei Diabetiker/innen dabei aber auch nicht belegbar). Eine weitere Meta-Analyse [321] (49 Studien, mehr als 73.000 Patient/innen) fand bei einem systolischen Ausgangsblutdruck von unter $140 \mathrm{~mm} \mathrm{Hg}$ lediglich einen Trend zu einer Schlaganfallreduktion, aber erhöhte kardiovaskuläre Mortalität, und bei einem erreichten systolischen BD von unter $130 \mathrm{~mm}$ Hg eine Schlaganfallreduktion. In der randomisierten ADVANCE Studie [322] (Perindopril-Indapamid versus Placebo) fand sich bei einem erreichten systolischen BD von $135 \mathrm{~mm} \mathrm{Hg}$ (versus $140 \mathrm{~mm} \mathrm{Hg}$ in der Placebogruppe) eine signifikante Reduktion von kardiovaskulärer und GesamtSterblichkeit. In der 2010 publizierten ACCORD Studie [323] (mehr als 4700 Patient/innen) wurden nach dem ersten Jahr im Mittel systolische Blutdruckwerte von $119 \mathrm{~mm} \mathrm{Hg}$ in der intensivierten Therapiegruppe versus $133 \mathrm{~mm} \mathrm{Hg}$ im Standardtherapiearm erreicht. Die intensive Blutdrucksenkung führte zu einer Reduktion von Schlaganfällen um $47 \%$, schwere kardiovaskuläre Ereignisse und die Mortalität waren in den Gesamt-Gruppen jedoch nicht unterschiedlich. Durch das $2 \times 2$-Design der ACCORD-Studie (jeweils intensive versus Standardtherapie von Glucose und Blutdruck) ist die Studie aber schwer interpretierbar, da Interaktionen der Therapiearme möglich sind. In mehreren Nachanalysen fand sich, dass Patient/ innen im Standard-Glukose-Arm von der intensiveren Blutdrucksenkung profitieren könnten [324, 325]. Bei intensivierter Therapie muss auf potentielle Nebenwirkungen wie Herzrhythmusstörungen, Hypo- und Hyperkaliämie und Hypotension geachtet werden, ebenso kann die GFR abnehmen. In der Subgruppe der Diabetiker/innen $(n=6400)$ in der INVEST Studie [326] (Patient/innen mit koronarer Herzerkrankung wurden mit Verapamil SR versus Atenolol und einem Blutdruckziel unter 130/85 mm Hg behandelt) 
war ein erreichter systolischer BD über $140 \mathrm{~mm} \mathrm{Hg} \mathrm{im}$ Vergleich zu unter $140 \mathrm{~mm}$ Hg mit vermehrten kardiovaskulären Ereignissen assoziiert. Ein systolischer BD unter $130 \mathrm{~mm} \mathrm{Hg}$ war im Vergleich zu über $130 \mathrm{~mm} \mathrm{Hg}$ erst nach einer verlängerten Nachbeobachtungszeit mit einer niedrigeren Mortalität assoziiert. In der SPRINT Studie waren keine Diabetiker/innen eingeschlossen. Eine post-hoc-Analyse von SPRINT an 3898 Patienten mit Prädiabetes [327] (definiert als Nüchternglukose $>100 \mathrm{mg} / \mathrm{dl}$ ) zeigte aber, dass bei diesen Patient/innen die intensive Blutdrucksenkung (<120 mm Hg systolisch, allerdings mit der unbeobachteten Office-Messung erhoben) ebenso vorteilhaft wie in der Gesamtstudie war. Systolische Werte unter $115 \mathrm{~mm} \mathrm{Hg}$ sollten vermieden, da hier die Gesamtsterblichkeit ansteigen kann [326].

Hinsichtlich des diastolischen Blutdrucks fand sich in der HOT-Studie [328] ein Vorteil einer Senkung mit dem Ziel unter $80 \mathrm{~mm} \mathrm{Hg}$ (erreicht wurden $81 \mathrm{~mm} \mathrm{Hg}$ ). In der UKPDS Studie [329] fand sich ein Vorteil für strengere Blutdruckeinstellung, erreicht wurde diastolisch $82 \mathrm{~mm} \mathrm{Hg}$. In der oben genannten ADVANCE Studie lag der diastolische BD in der Verum-Gruppe bei $75 \mathrm{~mm} \mathrm{Hg}$. Die oben angeführte Meta-Analyse [320] zeigte, dass ein erreichter diastolischer BD unter $80 \mathrm{~mm}$ Hg mit einer Reduktion aller untersuchter kardiovaskulärer Endpunkte einhergeht.

\section{Zielwerte für den Blutdruck}

Wir empfehlen als systolisches Office-Blutdruckziel bei Diabetes mellitus $130-139 \mathrm{~mm} \mathrm{Hg}$, bei guter Verträglichkeit ist ein niedrigerer Zielbereich von 120-129 mm Hg möglich. Der Zielbereich des diastolischen Blutdrucks beträgt unter $80 \mathrm{~mm} \mathrm{Hg}$.

\section{Maßnahmen zur Blutdrucksenkung}

\section{Lebensstilinterventionen}

Bei Diabetikern können Lebensstilmaßnahmen den BD bzw. die Blutdruckbehandlung positiv beeinflussen. Eine Gewichtsreduktion von ca. $8 \mathrm{~kg}$ kann bei übergewichtigen/adipösen Patient/innen mit Typ2-Diabetes nach einem Jahr die Blutdruckwerte um $5 / 2 \mathrm{~mm}$ Hg signifikant reduzieren [330]. Ein Gewichtsverlust $>15 \%$ senkte den systolischen BD in der LOOKAHEAD-Studie sogar um $10 \mathrm{~mm} \mathrm{Hg}$ [330]. Die empfohlenen Lebensstilmassnahmen entsprechen denen der Nicht-Diabetiker/innen.

\section{Antihypertensiva}

Die Blutdruckziele sind bei Diabetiker/innen oft schwierig zu erreichen [331]. Daraus ergibt sich die grundsätzliche Empfehlung, eine antihypertensive Therapie bei Diabetes mit einer Zweifach-Kombination zu beginnen. Diese beinhaltet einen ACEHemmer oder ARB und einen Kalziumantagonisten oder ein Thiaziddiuretikum. Prinzipiell sind alle grossen Substanzklassen (ACE-Hemmer, ARBs, Di- uretika vom Thiazid-Typ und Kalziumantagonisten vom Dihydropyridin-Typ) bei Diabetiker/innen zur Blutdrucksenkung einsetzbar, in einer grossen MetaAnalyse [319] waren sie zur Verhinderung kardiovaskulärer Endpunkte im Wesentlichen gleich gut wirksam. Eine weitere Meta-Analyse [320] bestätigte diese Ergebnisse und auch die Ausnahmen davon: Diuretika sind effektiver in der Verhinderung von Herzinsuffizienz, Kalziumantagonisten effektiver in der Verhinderung von Schlaganfällen und weniger effektiv in der Verhinderung von Herzinsuffizienz, Betablocker sind weniger effektiv in der Verhinderung von Schlaganfällen, ACE-Hemmer/ARBs sind effektiver in der Verhinderung von kardiovaskulären Ereignissen (Myokardinfarkt und Schlaganfall), der Gesamtsterblichkeit (grenzwertige Signifikanz) sowie dem Auftreten einer terminalen Niereninsuffizienz. Zusätzlich wirken ACE-Hemmer/ARBs am Besten in der Prävention und Hemmung einer Progression einer Albuminurie [332]. Wenn man die gesamte Evidenz in Betracht zieht, sollten ACE-Hemmer/ARBs Teil der initialen medikamentösen Blutdrucktherapie sein. Die Kombination von ACE-Hemmern und ARBs hat sich als nicht sinnvoll [333], eine Kombination dieser beiden Medikamentenklassen mit direkten Renin-Inhibitoren aufgrund der Nebenwirkungen als kontraindiziert [334] erwiesen.

\section{Antidiabetika und Blutdrucksenkung}

SGLT-2-Inhibitoren, GLP-1-Rezeptoragonisten und Pioglitazon senken den $\mathrm{BD}$, unabhängig davon, ob eine Hypertension oder eine Normotension vorliegt. Metformin, Insulin, Sulfonylharnstoffen und DPP-4Inhibitoren wirken nicht blutdrucksenkend.

Die in mehreren Metaanalysen berichteten blutdrucksenkenden Effekte betrugen für Glitazone (13 Studien) im Vergleich zu Placebo systolisch 4,9 und diastolisch 1,8 mm Hg [335]. Bei GLP-1-Rezeptoragonisten wurden für Exenatide (im Vergleich zu Placebo) eine Blutdruckreduktion von systolisch 5,2 mm $\mathrm{Hg}$ und diastolisch $5,4 \mathrm{~mm} \mathrm{Hg}$, für Liraglutid eine systolische Absenkung um 5,6 mm Hg berichtet [336]. Für SGLT-2-Inhibitoren (Canagliflozin, Dapagliflozin, Empagliflozin und Ertugliflozin; insgesamt 6 Studien) ließ sich eine signifikante 24-Stunden-Blutdruckreduktion systolisch um 3,8 mm Hg und diastolisch um 1,8 mm Hg nachweisen [337]. Die Blutdrucksenkung unter Empagliflozin ist unter Behandlung mit ACEHemmern/ARBs oder Diuretika tendenziell etwas stärker [338]. Die Blutdrucksenkungen korrelierten nicht mit dem Ausgangsblutdruck oder der Gewichtsabnahme. Der blutdrucksenkende Effekt der SGLT-2Inhibitoren ist in Hinblick auf Nephroprotektion und Kardioprotektion als günstig zu beurteilen.

In der EMPA-REG-Outcome-Studie [339] kam es unter Empagliflozin zu einer hochsignifikanten Senkung von kardiovaskulärem Tod, Gesamtmortalität, und Progression der Nierenerkrankung bei Patienten mit etablierter kardiovaskulärer Erkrankung. Der Blut- 
druckabfall von 135,3/76,6 mm $\mathrm{Hg}$ auf 131,3/75,1 mm Hg hatte keinerlei negativen Effekt. Diese Daten weisen darauf hin, dass Blutdruckwerte um $130 \mathrm{~mm} \mathrm{Hg}$ auch bei Hochrisikopatient/innen mit Typ-2-Diabetes nicht gefährlich sein dürften. In der CANVAS- und DECLARE-TIMI-58-Studie konnte zudem für Canagliflozin und Dapagliflozin (resp.) ein kardiovaskulärer Benefit, insbesondere eine Reduktion der Hospitalisierungs-Rate aufgrund einer Herzinsuffizienz, auch bei Patienten ohne manifeste kardiovaskuläre Erkrankung, aber mit multiplen kardiovaskuläre Risikofaktoren (Primärprävention) nachgewiesen werden [340, 341]. Bei älteren Patient/innen sollte bei einer Kombinationstherapie von Antihypertensiva und SGLT-2-Inhibitoren eine häufigere Blutdruckmessung erfolgen, um im Bedarfsfall die Zahl oder Dosis der Antihypertensiva zu reduzieren. Auch für GLP-1-Rezeptoragonisten liegen positive kardiovaskuläre Outcome-Daten vor. So konnte sowohl unter Lira-, Sema- als auch Dulaglutid eine signifikante Reduktion der Ereignisrate (3-Punkt-MACE aus kardiovaskulärem Tod, nicht-tödlichem Herzinfarkt und nicht-tödlichem Schlaganfall) gezeigt werden [342-344].

\section{Fact Box Blutdruck und Diabetes mellitus}

- Hypertonie ist sehr häufig bei Diabetiker/innen und mit einem hohen bzw. sehr hohen kardiovaskulären Risiko verbunden

- Blutdruckziel ist $130-139 / 70-79 \mathrm{~mm} \mathrm{Hg}$, bei guter Verträglichkeit und jüngerem Alter (<65 Jahren) 120-129 mm Hg systolisch

- Die medikamentöse Therapie sollte als Kombinationstherapie begonnen werden

- Ein ACE-Hemmer oder ein ARB sollte Teil der Initialtherapie sein

- Einige Antidiabetika-Klassen führen zu einer klinisch relevanten Blutdrucksenkung (SGLT-2-Inhibitoren, GLP-1-Rezeptoragonisten und Pioglitazon)

\section{Blutdruck und Nierenerkrankungen}

\section{Einleitung}

Hypertonie ist ein wesentlicher Risikofaktor für das Entstehen einer Nierenschädigung und ebenso für die Progression einer chronischen Niereninsuffizienz jedweder Ursache. Die Nieren können „Opfer“, das heißt Nierenschädigung durch Hypertonie, wie „Täter“, das heißt Auslöser einer Hypertonie mit allen sich daraus ergebenden Konsequenzen sein. Nach Diabetes ist die hypertensiv-vaskuläre Nephropathie die zweithäufigste Ursache für chronische Niereninsuffizienz $(\mathrm{CKD}=$ chronic kidney disease) und chronisch dialysepflichtiges Nierenversagen in Österreich [345, 346]. Hypertonie kann erstes Symptom einer sonst klinisch asymptomatisch verlaufenden, spezifischen Nierenerkrankung, z. B. Glomerulonephritis sein. In dieser Situation ist das Serum-Kreatinin ein eher insensitiver Marker, da durch Gegenregulationsmechanismen wie die tubuläre Sekretion von Kreatinin erst bei beträchtlicher Nierenfunktionseinschränkung ein Anstieg des Kreatinins im Serum erfolgt [52]. Suffiziente BD-Senkung, insbesondere durch ACEHemmer/ARBs kann ebenfalls einen Anstieg des Serumkreatinins um bis $\mathrm{zu} 30 \%$ bewirken, was aber ein funktionell-hämodynamischer Effekt durch Reduktion des glomerulären Filtrationsdruckes ist [347]. Die Langzeit-Auswirkungen dieses Phänomens sind unklar [348]. Die Stadieneinteilung der Niereninsuffizienz (Abb. 5) erfolgt nach Empfehlungen der Kidney Disease Improving Global Outcomes (KDIGO) Clinical Practice Guideline [349], deren hier zitierte Zusammenfassung auch praktische Tipps zur Zusammenarbeit Allgemeinmedizin - Nephrologie oder Häufigkeit von Kontrollen gibt.

\section{Anamnese und klinische Abklärung}

Wie auch bei nierengesunden Hypertoniker/innen dient die klinische Aufarbeitung der Diagnosestellung sowie der Einschätzung des Schweregrades der Hypertonie und ihrer Langzeitfolgen. Dabei kommt der Blutdruckmessung zentrale Bedeutung zu. Nach einer rezenten Übersicht soll bei Nierenpatient/innen bevorzugt eine out-of-office-Messung wie die Selbstmessung oder das ambulante 24-Stunden-Blutdruckmonitoring $(\mathrm{ABPM}=$ ambulatory blood pressure monitoring) zur Anwendung kommen [350]. Validierte Geräte mit oszillometrischer Messmethode und alle üblichen Empfehlungen zur korrekten Blutdruckmessung sind auch bei Nierenpatient/innen anzuwenden [351]. Bei CKD-Patient/innen geht eine eingeschränkte glomeruläre Filtrationsrate (GFR) und Albuminurie/Proteinurie häufiger mit maskierter Hypertonie einher, gehäuft fehlt die nächtliche Blutdruckabsenkung und es besteht ein höheres Risiko für Hypertonie-vermittelte Organschäden [350]. ABPM ist bei CKD prognostisch aussagekräftiger [352]: Bei 436 CKD-Patient/innen mit sowohl Ordinationsmessung als auch ABPM ergab sich bei einem systolischen ABPM-Tagesblutdruck $>145 \mathrm{~mm} \mathrm{Hg}$ ein dreifach erhöhtes Risiko für kardiovaskuläre Ereignisse und ein beinahe zweifach erhöhtes Risiko für dialysepflichtige Niereninsuffizienz oder Tod im Vergleich zu Patient/innen mit systolischem BD im Bereich 126-135 mm Hg. Wurde der Durchschnitt aus sechs Ordinationsmessungen berechnet, konnte diese prognostische Assoziation nicht erhoben werden.

Die in der Systolic Blood Pressure Intervention Trial (SPRINT) Studie [27] angewendete unbeobachtete automatisierte oszillometrische Ordinations-Blutdruckmessung (automated oscillometric blood pressure monitoring - AOBPM) ergab zu den ABPM-Tageswerten vergleichbare Ergebnisse [353]. Eine Substudie zu CKD-Patient/innen in SPRINT zeigt, dass aufgrund der kleinen Fallzahl auf Nierenpatient/innen keine sicheren Rückschlüsse gezogen werden können [354]. 
Abb. 5 Stadieneinteilung der chronischen Niereninsuffizienz (eGFR geschätzte glomeruläre Filtrationsrate, UACR HarnAlbumin-Kreatinin-Ratio, n.a. nicht anwendbar; Farben weisen auf das Risiko und Ausmaß hinsichtlich Mortalität, kardiovaskuläre Ereignisse, Progression der chronischen Nierenerkrankung etc.: Grün gering, gelb mäßig, orange $\sim$ hoch, rot $\sim$ sehr hoch). (Nach KDIGO [349])

\begin{tabular}{|c|c|c|c|c|c|}
\hline Stadium & & & A1 & A2 & A3 \\
\hline & $\begin{array}{l}\text { eGFR } \\
\mathrm{ml} / \mathrm{min} / 1.73 \mathrm{~m}^{2}\end{array}$ & $\begin{array}{l}\text { UACR } \\
\mathrm{mg} / \mathrm{g}\end{array}$ & $<30 \mathrm{mg} / \mathrm{g}$ & $\begin{array}{l}30- \\
300 \mathrm{mg} / \mathrm{g}\end{array}$ & $>300 \mathrm{mg} / \mathrm{g}$ \\
\hline G1 & $>90$ & & n.a. & & \\
\hline G2 & $60-89$ & & & & \\
\hline G3a & $45-59$ & & & & \\
\hline G3b & $30-44$ & & & & \\
\hline G4 & $15-29$ & & & & \\
\hline G5 & $<15$ & & & & \\
\hline
\end{tabular}

Die AOBPM ist gegenwärtig für CKD-Patient/innen nicht validiert.

\section{Nieren und Hypertonie}

Die Pathogenese einer Hypertonie bei Nierenerkrankungen hängt von der Art der Erkrankung (glomerulär versus vaskulär) und von der Erkrankungsdauer (akut versus chronisch) ab. Bei akuter Glomerulonephritis ist die Ursache überwiegend auf die Tendenz zur Volumenexpansion und die Natriumretention zurückzuführen [355]. Bei vaskulärer Erkrankung steht die Ischämie-induzierte Aktivierung des Renin-Angiotensin-Aldosteron-Systems (RAAS) im Vordergrund. Bei chronischer Niereninsuffizienz haben ca. $80-85 \%$ aller Betroffenen eine Hypertonie [356]. Die hohe Prävalenz ist auf multiple Faktoren zurückzuführen, wozu neben Natriumretention, RAAS-Aktivierung, auch ein erhöhter Sympathikotonus [357], ein sekundärer Hyperparathyreoidismus [358] oder eine verminderte NO-Synthese [359] eine Rolle spielen.

Die hypertensive (vaskuläre) Nephropathie als zweithäufigste Ursache von dialysepflichtigem Nierenversagen ist eine Ausschlussdiagnose (siehe Tab. 26). Daran zu denken ist bei eher jahrzehntelang bestehender Hypertonie, eingeschränkter eGFR $(e=$ estimated = geschätzt; Berechnung z. B. mittels CKDEPI-Formel [68]) und/oder Albuminurie geringen bis mittleren Ausmaßes, das heißt nach Albumin-Kreatinin-Ratio (UACR = urinary albumin creatinin ratio) $30 \mathrm{mg} / \mathrm{g}$ bis $2-3 \mathrm{~g} / \mathrm{g}$. Die Nierenfunktionsverschlechterung wie das Ausmaß der Albuminurie/Proteinurie sind nur langsam progredient. Im Harnbefund zeigen sich keine Elemente eines nephritischen Geschehens wie Erythrozytenzylinder oder dysmorphe Erythrozyten (dysmorphe Mikrohämaturie). Hinweisend ist eine hypertensive Retinopathie in der Funduskopie, Linksherz-Hypertrophiezeichen im EKG, eine Linksherzhypertrophie in der Echokardiographie und ev. eine Hyperuricämie. Andere Ursachen einer Niereninsuffizienz sind, soweit möglich, ausgeschlossen, eine Nierenbiopsie ist mangels therapeutischer Konsequenzen zumeist nicht erforderlich. Pathohistologisch zeigt sich eine Nephrosklerose, die letztlich zur Organverkleinerung und Schrumpfnieren führt, was sonographisch einfach nachweisbar ist.

\section{Antihypertensive Therapie}

\section{Nicht-pharmakologische Maßnahmen}

Prinzipiell gelten alle, für Hypertoniker empfohlenen Maßnahmen der Lebensstiländerung genauso für Patient/innen mit Niereninsuffizienz und Hypertonie. Allerdings gibt es dazu kaum Untersuchungen bei Patient/innen mit CKD. Eine Studie aus Japan an 2379 Patient/innen ergab ein positives Ergebnis [360]. Der Interventionsgruppe wurde zusätzlich zu ,standard-of-care' eine Beratung zu Lebensstilmodifikationen zuteil. Weiter erhielten die Patient/innen einen erklärenden Brief zu ihrem CKD-Status, der sie davon abhalten sollte, sich der Behandlung zu entziehen. Zuletzt erhielten ihre Hausärzte/Hausärztinnen Befundblätter mit Hinweisen, wie die Abstände zwischen Zielbereichen und aktuellen Befunden reduziert 
Tab. 26 Anamnese und klinische Befunde die die Diagnose einer hypertensiven Nephropathie wahrscheinlich machen

\begin{tabular}{l} 
Anamnestisch jahre-/jahrzehntelange Hypertonie \\
Hypertensive Retinopathie \\
Linksherz-Hypertrophie (EKG, Echokardiographie) \\
(Meist) langsam progredienter Anstieg von BUN und Serum-Kreatinin und \\
dementsprechend Abfall der eGFR \\
\hline Milde (bis $1 \mathrm{~g} / 24 \mathrm{~h}$ ), progredient zunehmende Proteinurie \\
\hline Hyperuricämie \\
\hline Unauffälliges Harnsediment (wenig Zellen, hyaline Zylinder) \\
Kein Hinweis auf andere Genese der Niereninsuffizienz \\
\hline
\end{tabular}

werden könnten. Die Interventionsgruppe wies eine signifikant bessere Adhärenz auf und die Zusammenarbeit der Allgemeinmediziner/innen mit Nephrolog/ innen war signifikant besser. Im Interventionskollektiv zeigte sich ein Trend zu einer langsameren Abnahme der Nierenfunktion, ein Befund, der in der Gruppe mit Stadium G3 Signifikanzniveau erreichte (Kontrollgruppe $-2,4 \pm 5,9 \mathrm{ml} / \mathrm{min} / 1,73 \mathrm{~m}^{2} / \mathrm{Jahr}$ versus Interventionsgruppe $-1,9 \pm 4,4 \mathrm{ml} / \mathrm{min} / 1,73 \mathrm{~m}^{2} / \mathrm{Jahr}$; $p=0,03$ ) [360]. Dies darf zumindest als Hinweis darauf gewertet werden, dass Lebensstilmodifikation auch für CKD-Patient/innen Sinn macht.

\section{Salzkonsum}

Unter den Maßnahmen zur Modifikation der Ernährung nimmt der tägliche Salzkonsum eine besondere Stellung ein. In der Zusammenschau einer rezent abgehaltenen Expert/innen-Konferenz wird beim Management möglicher Komplikationen glomerulärer Erkrankungen als erstes auf die Hypertonie eingegangen und dabei insbesondere eine Beschränkung der Natriumzufuhr auf $1500 \mathrm{mg}$ pro Tag (entspricht knapp $4 \mathrm{~g}$ Kochsalz/d) als günstig betont [361]. Eine Meta-Analyse von 17 Studien an Hypertoniker/innen mit moderater Reduktion der Salzzufuhr (im Mittel $-4,6 \mathrm{~g} / \mathrm{d}$ ) und mindestens 4-wöchiger Dauer konnte zeigen, dass damit eine Blutdrucksenkung von systolisch/diastolisch 5/3 mm Hg erreicht werden kann [362]. Allerdings wurde nicht untersucht, inwieweit das Ergebnis auch für CKD-Patient/innen gilt. Eine kleine, aber sorgfältig durchgeführte Studie aus Groningen untersuchte Patient/innen mit Niereninsuffizienz unterschiedlicher Genese und Proteinurie hinsichtlich des Effekts einer Salzrestriktion sowie Gabe von Hydrochlorothiazid auf die antiproteinurische Wirkung von Losartan [363]. Zum einen wird der bekannte Effekt auf die höhere Wirksamkeit einer RASBlockade unter Salzrestriktion bestätigt [364]. Vor allem aber zeigt die Studie, dass der BD in der PlaceboGruppe von im Mittel 143/86 unter Salzbeschränkung (Natriumzufuhr gemessen an der Natriumausscheidung im 24-h-Urin: hoch $196 \mathrm{mmol} \mathrm{Na} / \mathrm{d} \sim$ ca. 11,5g Salz/d versus niedrig $92 \mathrm{mmol} \mathrm{Na/d} \sim$ ca. 5,4 g Salz/d) auf 137/83 mm Hg signifikant absank [363]. Die Proteinurie wurde unter Salzrestriktion um 22\% reduziert.
Eine rezente Übersicht kommt zusammenfassend zum Schluss, dass Beschränkung der Salzzufuhr sich protektiv hinsichtlich kardiovaskulärer wie renaler Ereignisse auswirkt. Allerdings sollten größere und vor allem länger dauernde Studien durchgeführt werden, um diese positive Wirkung endgültig abzusichern [365].

In der Entwicklung einer Hypertonie dürfte neben übermäßigem Salzkonsum auch Fructose eine Rolle spielen [366], beide Nahrungsbestandteile sind oft gemeinsam in relativ hoher Konzentration in Fertigund Halbfertigprodukten der Nahrungsmittelindustrie enthalten. Über direkte Wirkung, Effekte über Insulin-Resistenz-assoziierte Kaskaden und andere Wege fördert Fructose hohen BD und in Tier-Studien mit verminderter Fructose-Zufuhr konnte bereits eine BD-senkende Wirkung gezeigt werden. Generell ist die frische Zubereitung der Nahrung mit Kontrolle über die Zutaten (z.B. Salz) vorteilhaft, auch hinsichtlich BD.

\section{Pharmakotherapie}

Antihypertensiva-Substanzklassen und Pharmakokinetik und -dynamik bei chronischer Niereninsuffizienz

Insbesondere bei Niereninsuffizienz ist die Kenntnis und Berücksichtigung pharmakologischer und pharmakodynamischer Eigenschaften der Antihypertensiva von Vorteil [367]. Bei allen überwiegend renal eliminierten Antihypertensiva ist an eine verlängerte Halbwertszeit der Substanzen im Plasma und damit an die erforderliche Dosisanpassung zu denken.

Hemmer des Angiotensin-konvertierenden Enzyms (ACE-Hemmer) bewirken eine Verminderung der Aldosteron-Sekretion und Bildung von Angiotensin II und vermindern damit den peripheren Gefäßwiderstand, ähnlich wie die Angiotensin-II-Rezeptoren-Blocker (ARB). Es kommt zur Erweiterung der efferenten Arteriole im Glomerulum mit Senkung des intraglomerulären Perfusionsdruckes, was den wahrscheinlich zentralen Mechanismus der Nephroprotektion dieser Substanzgruppe darstellt. Zu erwähnen ist natürlich auch der antiproteinurische Effekt, wobei die Datenlage widersprüchlich ist. Eine Meta-Analyse mit gesamt 1860 Patient/innen sieht bei Proteinurie $<500 \mathrm{mg} / \mathrm{d}$ bzw. geringem Progressionsrisiko keinen Vorteil durch eine ACE-Hemmer-Therapie [368]. Für Patient/innen mit hypertensiver Nephropathie und Albuminurie bis $500 \mathrm{mg} / \mathrm{d}$ ist also keine harte Indikation für ACE-Hemmer gegeben. Anders ist die Situation bei Proteinurie $>500 \mathrm{mg} / \mathrm{d}$ und hohem Progressionsrisiko, wo sehr wohl ein signifikanter Vorteil besteht. $\mathrm{Zu}$ beachten ist, dass das RAAS-System die Ausscheidung von Kalium im distalen Tubulussystem bewirkt, weshalb es bei Blockade zur Hyperkaliämie kommt, ein Effekt, der bei CKD ab Stadium 3b nochmals häufiger wird [369, 370]. Bei dialysepflichtigen Patient/innen genügt es bei länger wirksamen ACE- 
Hemmern wie Lisinopril für die gewünschte BD-senkende Wirkung je eine Dosis nach Hämodialyse dreimal pro Woche zu verabreichen [371].

Dihydropyridin-Kalziumkanal-Blocker (DHP-Kalziumantagonisten) bewirken eine reduzierte Muskelkontraktilität glatter Muskulatur in den Gefäßen. Die Datenlage für DHP-Kalziumantagonisten bei CKDPatient/innen ist dünn, wenngleich sie beispielsweise in ACE-Hemmer- [372] oder ARB-Studien [370] als Vergleichssubstanz bei CKD-Patient/innen getestet wurden. Es gibt Studien an Dialysepatient/innen, die zeigen, dass Amlodipin sicher und BD-senkend und wahrscheinlich günstig hinsichtlich kardiovaskulärer Ereignisse wirkt [373]. Aufgrund hepatischer Elimination werden DHP-Kalziumantagonisten bei CKD normal dosiert. Erwähnenswert ist die bekannte Nebenwirkung peripherer Ödeme. Bei gleichzeitiger Gabe von Diuretika oder auch ACE-Hemmer/ARBs kann dies zumindest bei einem Teil der Patient/innen ausgeglichen werden.

Thiazid- und Thiazid-artige Diuretika wirken auf den Na-Cl-Cotransporter im distalen Tubulus der Nephrone, wo etwa $5 \%$ der Natrium-Rückresorption stattfindet. Die durch Thiazide ausgelöste Natriurese bewirkt akut eine Reduktion des Extrazellulärvolumens, des venösen Rückstroms zum Herzen und damit einen verminderten kardialen Auswurf. Bei regelmäßiger Anwendung normalisiert sich das Herzminutenvolumen und der periphere Widerstand vermindert sich, was die Blutdrucksenkung aufrecht hält. Bei Niereninsuffizienz besteht eine chronische Volumenexpansion, eine erhöhte kardiale Auswurfleistung mit erhöhtem peripheren Gefäßwiderstand und dadurch Hypertonie [374]. Entgegen früheren Ansichten sind Thiaziddiuretika auch bei fortgeschrittener Niereninsuffizienz bis zu einem gewissen Grad wirksam, was sich aus zumindest 5 randomisierten Studien mit Thiaziden allein oder kombiniert mit Schleifendiuretika ablesen lässt [375]. Die Aussage der KDIGO Blutdruck-Arbeitsgruppe, dass die Wirkung der Thiazide auf die Flüssigkeitsretention jenseits einer GFR von $30-50 \mathrm{ml} / \mathrm{min} / 1,73 \mathrm{~m}^{2}$ zwar abnimmt, deren günstiger Effekt auf den BD aber erhalten bleibt [376], spricht für die Fortführung dieser Medikation. In der praktischen Erfahrung vieler Nephrologen wird bei Bedarf das Thiazid nicht unbedingt abgesetzt, sondern mit Schleifendiuretika kombiniert. Zur Klärung dieser Frage wurde 2018 eine doppel-blinde, randomisierte, kontrollierte Studie bei Patient/innen mit Stadium G4 und der Frage BD-Wirksamkeit begonnen [377]. $\mathrm{Zu}$ bedenken sind die bekannten Nebenwirkungen wie gestörte Glukosetoleranz, Hyponatriämie und Hypokaliämie, oder Hyperuricämie. Bei terminaler Niereninsuffizienz sind Thiazide unwirksam. Es ist darauf zu verweisen, dass die wesentlichen Studien zur Etablierung von Thiaziden in der antihypertensiven Therapie mit Chlorthalidon durchgeführt wurden. Durch Studien und Meta-Analysen abgesichert, wirken die Thiazid-artigen Diuretika wie Chlorthalidon bei ver- gleichbarem Nebenwirkungsprofil signifikant stärker BD-senkend als Hydrochlorothiazid [214, 378, 379], welches Bestandteil vieler Fixkombinationen ist. Eine Re-Analyse der Antihypertensive and Lipid-Lowering Treatment to Prevent Heart Attack Studie (ALLHAT) bestätigt auch im Hinblick auf renale Endpunkte die positive Wirkung von Chlorthalidon [380]. Es gibt sogar eine Studie im crossover-Design an 49 Patient/ innen mit systolischem $\mathrm{BD}>140 \mathrm{~mm} \mathrm{Hg}$ nach Nierentransplantation, wo Chlorthalidon (12,5-25 mg) mit Amlodipin (5-10 mg) verglichen wurde [381]. Beide Substanzen waren vergleichbar BD-senkend. Chlorthalidon bewirkte außerdem eine Reduktion der Proteinurie um 30\%, eine Verminderung von Ödemen, jedoch kam es temporär zu einem Anstieg der Harnsäure und des HbAlc sowie zu einer Transplantatfunktionseinschränkung. Die Autoren fassen zusammen, dass Chlorthalidon bei Nierentransplantierten ähnlich wirksam ist wie Amlodipin [381].

Die Bezeichnung „Schleifendiuretikum“ leitet sich vom Wirkort, dem Na-K-2Cl-Cotransporter im dicken aufsteigenden Teil der Henle'schen Schleife der Nephrone her. Dieser Transporter sorgt für etwa $25 \%$ der Natrium-Rückresorption. Schleifendiuretika sind aufgrund ihrer kurzen Wirkdauer als Antihypertensiva wenig geeignet, zur symptomatischen Therapie von Ödemen bei CKD-Patienten aber nicht wegzudenken. Mit Zunahme der Niereninsuffizienz sind steigende Dosen erforderlich, auch eine Kombination mit Thiaziden kann sinnvoll und notwendig sein.

Aldosteron als Mineralocorticoid gelangt über die basolaterale Membran der Zellen der Sammelrohre nach intrazellulär, bindet sich an den Mineralocorticoidrezeptor, transloziert dann in den Zellkern, wo es die Genexpression, insbesondere eine Kaskade von Schritten reguliert, die für den Umbau und die Degradation von epithelialen Natriumkanälen an der apikalen Zellmembran zuständig sind. Mineralocorticoidrezeptoren-Blocker $(\mathrm{MRA}=$ mineralocorticoid receptor antagonist; z. B. Spironolacton, Eplerenon; in nächster Zeit ev. auch kommend Finerenone) hemmen kompetitiv die Bindung von Aldosteron an seinen Rezeptor, was letztlich zu einer schnelleren Degradierung der Natriumkanäle führt und somit eine verminderte Natrium-Rückresorption bei gleichzeitig verminderter Kalium-Exkretion bewirkt. Daraus ergibt sich die, vor allem bei CKD-Patienten problematische, mögliche Nebenwirkung der Hyperkaliämie. MRA haben vor allem in der Behandlung der resistenten Hypertonie Bedeutung [225] (siehe Abschn. 23).

Exkurs Hyperkaliämie: Bezüglich der häufigen Hyperkaliämie unter MRA und/oder ACEHemmern/ARBs ergeben sich rezent neue Möglichkeiten. Patient/innen mit Hyperkaliämie sollten eine sofortige Ernährungsberatung (Kalium-reduzierte Kost, das heißt weniger Obst, Gemüse, Salat, Fruchtund Gemüsesäfte, bei Salat- und Gemüsezubereitung „Auswässern“ durch Einlegen in Wasser bzw. Kochen in reichlich Wasser mit Verwerfen des Kochwassers 
usw.) erhalten und müssen kurzfristig kontrolliert werden. Das Vorliegen einer metabolischen Azidose ist auszuschließen, weil dann bei CKD die Therapie zuerst in einer Azidose-Korrektur und damit Behebung der Shiftstörung beruht. Ist eine medikamentöse Korrektur der Hyperkaliämie erforderlich, so stehen Natrium-Poylstyren-Sulfonat als Austauscherharz [382] oder neu Patiromer, ein nicht-absorbierbares Kalium-bindendendes Polymer mit Kalzium als Austauscherion [383] zur Wiederherstellung bzw. zur Aufrechterhaltung einer Normokaliämie zur Verfügung. Autor/innen aus Großbritannien und Schweden errechneten in einem Modell, dass wenn es gelingt eine Normokaliämie zu gewährleisten, die Beibehaltung einer Therapie mit ACE-Hemmern/ARBs bei CKDPatient/innen eine Zunahme der Lebenserwartung um 2,36 Jahre, eine Verzögerung des Eintritts der Dialysepflichtigkeit um 5,4 Jahre und einen Zugewinn an ,quality-adjusted life-years' von 1,02 QALYs bewirkt [384]. $\mathrm{Zu}$ betonen ist, dass dieses Rechenmodell erst einer Verifizierung in vivo bedarf. Einzige Alternative ist, ACE-Hemmer/ARBs abzusetzen. Natrium-Polystyrene-Sulfonat bewirkte in einer kleinen Studie nach 7 Tagen Therapie eine Abnahme des Serum-Kaliums im Mittel von $1 \mathrm{mmol} / \mathrm{l}$ und das Erreichen einer Normokaliämie in $78 \%$ der Patient/ innen gegenüber $38 \%$ mit Placebo [382]. Auch wenn in der persönlichen Erfahrung eine Langzeit-Therapie möglich ist, gibt es dazu keine Studien. Außerdem weist ein systematischer Review auf teils schwere gastrointestinale Nebenwirkungen mit Colonnekrosen hin [385]. Eine weitere Kalium-bindende und damit Kalium-entfernende Substanz, die möglicherweise bald zur Aufrechterhaltung von Normokaliämie zur Verfügung stehen wird, ist Natrium-ZirconiumCyclosilicat, hier liegen Erfahrungen mit einjähriger Behandlungsdauer vor [386].

Pitt und Mitarbeiter/innen konnten bei Patient/ innen mit Indikation für die Gabe von Spironolacton aufgrund Herzinsuffizienz und gleichzeitig einer eGFR $<60 \mathrm{ml} / \mathrm{min} / 1,73 \mathrm{~m}^{2}$ zeigen, dass durch begleitende Gabe von Patiromer bei allen 63 eingeschlossenen Patient/innen die Verabreichung von $50 \mathrm{mg}$ Spironolacton möglich war und das SerumKalium im Normbereich gehalten werden konnte [387]. Eine weitere Studie an Zentren in Europa schloss insgesamt 304 Patient/innen mit einer mittleren eGFR von $40 \mathrm{ml} / \mathrm{min} / 1,73 \mathrm{~m}^{2}$ und Diabetes mit milder $(5,0$ bis $<5,5 \mathrm{mmol} / \mathrm{L})$ und moderater (5,5-6,0 mmol/L) Hyperkaliämie ein [388]. Es wurde gezeigt, dass mit Patiromer die ACE-Hemmer/ARBTherapie auch über ein Jahr bei Normokaliämie durchführbar war, was angesichts der Nephroprotektion durch ACE-Hemmer/ARBs einen Vorteil darstellt. Ein Expert/innen-Konsensus der European Society of Cardiology kommt zum Schluss, dass die Fortführung und Ausdosierung von ACE-Hemmern/ARBs und MRAs einen Vorteil darstellt und eine Normokaliämie gegebenenfalls durch Ernährungsberatung und
Gabe Kalium-senkender Medikamente herbeigeführt werden soll [389].

Zentral wirkend Sympatholytika (zentrale AlphaAgonisten) stimulieren Alpha-2-Rezeptoren im Hirnstamm und vermindern damit den Sympathikotonus, was bei CKD-Patient/innen eine wichtige Rolle spielt [390]. Vertreter dieser Substanzgruppe sind Clonidin und Moxonidin oder Rilmenidin, welche bei CKDPatient/innen mit schwer einstellbarer Hypertonie immer noch eine gewisse Rolle spielen [391], auch wenn Nebenwirkungen wie Mundtrockenheit, Sedierung oder Bradykardie und - insbesondere bei zu hoher Dosierung - auch Herzinsuffizienz zum Absetzen zwingen können.

Betablocker, die als Antihypertensiva eingesetzt werden, wirken vor allem auf $\beta 1$-adrenerge Rezeptoren, die überwiegend im Myokard exprimiert werden. Neuere Substanzen wie Carvedilol und Nebivolol mit kombiniert alpha- und beta-blockierender Wirkung haben auch vasodilatierende Wirkung. Der exakte Wirkmechanismus ist ungeklärt, die Verminderung des peripheren Widerstandes dürfte aber eine Rolle spielen [392]. Für CKD-Patient/innen ist nachgewiesen, dass eine Sympathikus-Überaktivität besteht [390, 393], weshalb $\beta$-Blocker bei CKD vielleicht mehr Bedeutung haben als bei Hypertoniker/innen mit anderen Comorbiditäten.

Alpha-Blocker wie Doxazosin [394] oder Urapidil bewirken über selektive Blockade der Alpha1-Adrenozeptoren in den Gefäßen eine Abnahme des systemischen Gefäßwiderstandes. Daneben bestehen möglicherweise günstige Auswirkungen auf verschiedene metabolische Parameter. Sie können bei Niereninsuffizienz normal dosiert werden. Die Wirksamkeit wurde etwa bei Diabetiker/innen mit CKD nachgewiesen [394], wenngleich die Substanz in ALLHAT weniger wirksam war als Chlorthalidon [380]. Wegen vermehrt Fällen von Herzinsuffizienz wurde der Alpha-BlockerArm in ALLHAT ja frühzeitig beendet. Zu beachten ist die Möglichkeit von Tachykardie, weshalb in dieser Situation die Kombination mit Betablockern empfohlen wird.

Minoxidil ist ein direkter Vasodilatator und bewirkt einen Kalium-Efflux aus den glatten Muskelzellen der Gefäßwände über Hemmung bestimmter KaliumKanäle, was eine Relaxation zur Folge hat. Neben dem gewünschten Effekt der arteriolären Dilatation kann es zu Reflextachycardie und Volumsretention mit Ödemen, eventuell auch als besonders problematisch zu einem Pericarderguss kommen. Dosisabhängig kommt es auch zu Hirsutismus, was eine Anwendung bei Frauen fast unmöglich macht. Minoxidil wie auch Hydralazin als weiterer direkter Vasodilatator sind zwar sehr effektiv in der BD-Senkung, kommen wegen der Nebenwirkungen jedoch nur als letzte Alternative bei schwerst einstellbarer Hypertonie zum Einsatz. So konnte in einer retrospektiven Kohortenstudie an 54 Patient/innen mit einer eGFR im Mittel von $34,1 \mathrm{ml} / \mathrm{min} / 1,73 \mathrm{~m}^{2}$, The- 
rapie-refraktärer Hypertonie und stationärer Aufnahme wegen hypertensiver Notfälle eine signifikante Blutdruckreduktion von 162,4 $\pm 15,1 / 83,2 \pm 12,7$ auf $135,8 \pm 12,2 / 72,8 \pm 6,9 \mathrm{~mm} \mathrm{Hg}$ erzielt werden [395]. Neben der nur retrospektiven Beobachtung ist dieser Studie allerdings eine nur kurze Nachbeobachtung anzukreiden. Da Minoxidil zuerst in der Leber in den aktiven Metaboliten umgewandelt wird und später zu ca. $20 \%$ über die Nieren ausgeschieden wird, erklärt dies möglicherweise die lange Wirkdauer.

\section{Therapeutisches Vorgehen}

Wie bei jedem Patienten/jeder Patientin mit Hypertonie sind in der Initialtherapie bei Patient/innen mit CKD Substanzklassen mit nachgewiesener Wirkung auf klinische Ereignisse wie Insult, Myokardinfarkt, Herzinsuffizienz, Verhinderung einer Nierenschädigung und Tod zu bevorzugen [116]. Bei CKD-Patient/ innen kommt als besonderes Ziel auch noch die Progressionsverzögerung der Niereninsuffizienz hinzu. Eine systematische Literaturrecherche und MetaAnalyse von letztlich 11 Studien und insgesamt 9287 Patient/innen kommt zum Ergebnis, dass im Vergleich zur Standardtherapie mit intensiver BD-Reduktion eine Nephroprotektion erreicht wird, vor allem bei Patient/innen mit Proteinurie [396]. Eine weitere Meta-Analyse von 18 Studien mit 15.924 Patient/ innen mit CKD Stadium 3-5 und einem systolischen Ausgangs-BD von $148 \mathrm{~mm} \mathrm{Hg}$ sowie jeweils 2 Therapie-Vergleichsgruppen konnte zeigen, dass eine Senkung des Blutdrucks auf $132 \mathrm{~mm} \mathrm{Hg}$ im Vergleich zu $140 \mathrm{~mm} \mathrm{Hg}$ eine um $14 \%$ signifikant reduzierte Mortalität jedweder Ursache bewirkte [397].

Nachdem Patient/innen mit CKD zumeist einen höheren Schweregrad der Hypertonie aufweisen, empfiehlt sich der Beginn mit ACE-Hemmern/ARBs [367] und schon initial eine Kombination mit DHPKalziumantagonisten oder Thiaziden mit langer Wirkdauer. Ein Bericht der National Kidney Foundation über den Einsatz von ACE-Hemmern/ARBs bei fortgeschrittener Niereninsuffizienz kommt zum Schluss, dass individualisierte Therapiepläne zur Anwendung kommen sollten, wo bei eGFR-Abfall um mehr als $20 \%$ bei gleichzeitig fehlendem Rückgang der Proteinurie diese Medikation auch wieder abgesetzt werden sollte [398]. Die Autor/innen folgern aber, dass mehr Forschung erforderlich ist, um den Stellenwert der ACEHemmer/ARBs bei fortgeschrittener Niereninsuffizienz besser beurteilen zu können. Eine interessante Analyse von 3939 Teilnehmer/innen der Chronic Renal Insufficiency Cohort (CRIC) zeigt auf, dass mit Progression der Niereninsuffizienz vom Stadium 2-3 zum Stadium 4-5 der Einsatz von ACE-Hemmern/ ARBs abnahm, der von Betablockern und Kalziumantagonisten zunahm, wobei unter ACE-Hemmern/ ARBs sowohl ein geringeres Risiko von Herzinsuffizienz wie auch Tod zu finden war [399]. Diese Erkenntnis spricht also dafür, ACE-Hemmer/ARBs konsequent einzusetzen. Dabei besteht zwischen den verschiedenen Medikamenten innerhalb der ACEHemmer/ARBs nach einer Netzwerk-Meta-Analyse von 100 Studien mit gesamt 22.365 Patient/innen mit diabetischer Nierenerkrankung kein signifikanter Unterschied hinsichtlich Wirkung auf das Eintreten einer terminalen Niereninsuffizienz, Verdopplung des Serumkreatinins, Normalisierung, Reduktion oder Progression einer Albuminurie/Proteinurie, alle zeigten günstige antiproteinurische Wirkung [400].

Diuretika sind vor allem bei Volumsüberladung sinnvolle Kombinationspartner. Nachdem circa 2/3 der Hypertoniker/innen mehr als 2 Medikamente benötigen, um ihr Blutdruckziel zu erreichen, kommen ohnedies häufig alle drei genannten Substanzklassen und je nach individueller Situation auch andere Substanzklassen wie Betablocker, Alphablocker, zentrale Sympatholytika oder Vasodilatatoren zum Einsatz. Durch die gleichzeitige Verwendung von Schleifendiuretika oder durch die Gabe Kalium-bindender Substanzen zur Gewährleistung einer Normokaliämie bekommen auch MRA wieder eine neue Bedeutung bei CKD-Patient/innen, vor allem bei resistenter Hypertonie oder gleichzeitig bestehender Herzinsuffizienz.

$\mathrm{Zu}$ beachten ist, dass bei CKD ganz allgemein eine Tendenz zu gehäuft auftretenden Nebenwirkungen entsprechend Fachinformation besteht und dass es bei Kombinationstherapien zur Kumulation von Nebenwirkungen kommen kann. Nicht sinnvoll ist die Kombination von ACE-Hemmern mit ARBs (oder Renin-Antagonisten) [401].

Nachdem viele Substanzklassen zur antihypertensiven Therapie zur Verfügung stehen, soll eine möglichst individualisierte Therapie, beginnend mit ein bis meist schon zwei Substanzen, konsequenter Dosis-Steigerung und/oder Kombination mit weiteren Wirkstoffklassen bis zur Erreichung des Therapieziels durchgeführt werden. $\mathrm{Zu}$ berücksichtigen sind Alter, Ausmaß der individuell antiproteinurischen Wirkung von ACE-Hemmern/ARBs, Anamnese oder prospektive Einschätzung hinsichtlich eines akuten Nierenversagens (akut-auf-chronisches Nierenversagen) und Geschwindigkeit der Nierenfunktionsabnahme [398]. ,Shared decision-making،, die Berücksichtigung sowohl der Patient/innen-Meinung und Patient/innenWünsche wie die ärztlichen Empfehlungen sollen zu einer gemeinsamen Behandlungsstrategie und einem Konsens über das nachfolgende Therapiemonitoring führen. Eine Zusammenfassung der wesentlichsten Merkmale von Antihypertensiva bei CKD findet sich in Tab. 27 (adaptiert nach [399]).

\section{Zielwerte}

Wie schon weiter oben ausgeführt bewirkt eine intensivierte Blutdrucksenkung eine Nephroprotektion mit verlangsamter Progression [396] wie auch eine Reduktion der Mortalität [397]. Frage ist, was „in- 
Tab. 27 Antihypertensiva bei chronischer Niereninsuffizienz - Indikationen und Beachtenswertes. (Adaptiert nach [399])

\begin{tabular}{|c|c|c|c|c|}
\hline Substanzklasse & Indikation bei CKD & Andere mögliche Indikationen & Häufige Nebenwirkungen & Potentielle Kontraindikationen \\
\hline ACE-Hemmer & $\begin{array}{l}\text { Reduktion der Proteinurie; Nephro- } \\
\text { protektion }\end{array}$ & $\begin{array}{l}\text { Herzinsuffizienz mit reduzierter } \\
\text { Auswurfleistung; nach Myo- } \\
\text { cardinfarkt }\end{array}$ & $\begin{array}{l}\text { Husten, Angioödem, Hyperkali- } \\
\text { ämie, Leukopenie, Anämie }\end{array}$ & $\begin{array}{l}\text { Schwangerschaft, beidseitige } \\
\text { Nierenarterienstenose }\end{array}$ \\
\hline $\begin{array}{l}\text { Angiotensin- } \\
\text { Rezeptoren- } \\
\text { Blocker }\end{array}$ & $\begin{array}{l}\text { Reduktion der Proteinurie; Nephro- } \\
\text { protektion }\end{array}$ & $\begin{array}{l}\text { Harnsäure-Senkung, Gicht, sonst } \\
\text { wie ACE-Hemmer }\end{array}$ & $\begin{array}{l}\text { Husten (Seltener als mit ACE-Hem- } \\
\text { mern), Angioödem, Hyperkaliämie }\end{array}$ & $\begin{array}{l}\text { Schwangerschaft, beidseitige } \\
\text { Nierenarterienstenose }\end{array}$ \\
\hline $\begin{array}{l}\text { Dihydropyridin- } \\
\text { Kalziumkanal- } \\
\text { Blocker }\end{array}$ & Reduktion der Proteinurie & $\begin{array}{l}\text { Raynaud-Symptomatik, Ösopha- } \\
\text { gusspasmen }\end{array}$ & Beinödeme, Gingiva-Hypertrophie & - \\
\hline $\begin{array}{l}\text { Thiazid- } \\
\text { Diuretika }\end{array}$ & $\begin{array}{l}\text { Volumsüberladung, in Kom- } \\
\text { bination mit ACE-Hemmern/ } \\
\text { Angiotensinrezeptoren-Blockern } \\
\text { günstig bei Proteinurie }\end{array}$ & $\begin{array}{l}\text { Nierenstein-Prävention (Hyper- } \\
\text { kalziurie) }\end{array}$ & $\begin{array}{l}\text { Hyperurikämie, Hyperkalziämie, } \\
\text { Hyponatriämie, Hypokaliämie, } \\
\text { Hyperglykämie (bei Langzeit-An- } \\
\text { wendung) }\end{array}$ & Gicht, Hyperkalziämie \\
\hline $\begin{array}{l}\text { Schleifen- } \\
\text { diuretika }\end{array}$ & Volumsüberladung & Herzinsuffizienz, Hyperkalziämie & $\begin{array}{l}\text { Hörverlust, Hypokaliämie, Hypokal- } \\
\text { ziämie, Hyponatriämie }\end{array}$ & $\begin{array}{l}\text { Gicht, Sulfonamid-assoziierte } \\
\text { Hypersensitivität }\end{array}$ \\
\hline $\begin{array}{l}\text { Mineralo- } \\
\text { corticoid- } \\
\text { rezeptoren- } \\
\text { Blocker }\end{array}$ & Reduktion der Proteinurie & $\begin{array}{l}\text { Leberzirrhose mit Aszites, Po- } \\
\text { lyzystisches Ovar-Syndrom, } \\
\text { Hyper-aldosteronismus }\end{array}$ & $\begin{array}{l}\text { Hyperkaliämie, metabolische } \\
\text { Azidose, Gynäkomastie }\end{array}$ & Schwere Hyperkaliämie \\
\hline $\begin{array}{l}\text { Zentral wirken- } \\
\text { de Sympatholyti- } \\
\text { ka }\end{array}$ & $\begin{array}{l}\text { Kombinationspartner zur Blut- } \\
\text { drucksenkung }\end{array}$ & - & $\begin{array}{l}\text { Sedierung, Mundtrockenheit, } \\
\text { Bradycardie } \\
\text { Rebound-Hypertonie }\end{array}$ & Depression \\
\hline $\begin{array}{l}\text { Betablocker } \\
\text { selektiv }\end{array}$ & - & $\begin{array}{l}\text { Herzinsuffizienz, Vorhofflimmern, } \\
\text { Migräne, essentieller Tremor, } \\
\text { Angstzustände, Angina pectoris }\end{array}$ & $\begin{array}{l}\text { Bradycardie, Hyperkaliämie, } \\
\text { Mattigkeit, Depression, erektile } \\
\text { Dysfunktion }\end{array}$ & $\begin{array}{l}\text { Asthma bronchiale, chro- } \\
\text { nisch-obstruktive Lungener- } \\
\text { krankung; 2- und 3-gradiger } \\
\text { Herzblock }\end{array}$ \\
\hline $\begin{array}{l}\text { Kombinierte } \\
\text { Alpha-Beta-Blo- } \\
\text { cker }\end{array}$ & - & Herzinsuffizienz, Vorhofflimmern & $\begin{array}{l}\text { Bradycardie, Hyperkaliämie, } \\
\text { Mattigkeit, Depression, erektile } \\
\text { Dysfunktion }\end{array}$ & 2- und 3-gradiger Herzblock \\
\hline Alphablocker & - & Prostatahyperplasie & Orthostase-Reaktion & - \\
\hline Vasodilatatoren & - & - & $\begin{array}{l}\text { Kopfschmerz, Tachycardie, } \\
\text { Hirsutismus, Ödeme, Pericarder- } \\
\text { guss, Lupus-artiges Syndrom }\end{array}$ & $\begin{array}{l}\text { Post-Myocardinfarkt, Herzin- } \\
\text { suffizienz }\end{array}$ \\
\hline
\end{tabular}

tensivierte Blutdrucksenkung“ genau bedeutet. Eine rezente Übersicht kommt zum Ergebnis, dass ein $\mathrm{BD}<130 / 80 \mathrm{~mm} \mathrm{Hg}$ einen vernünftigen, durch Daten abgesicherten BD-Zielwert für Patient/innen mit CKD darstellt [402]. Eine Expert/innenkonferenz von KDIGO setzt den BD-Zielwert insbesondere bei Patient/innen mit Proteinurie $>1 \mathrm{~g} / \mathrm{d}$ trotz teils kontroversieller Datenlage mit 125/75 mm Hg noch niedriger an [361]. Die Subgruppenanalyse der CKD-Patient/innen des Systolic Blood Pressure Intervention Trial (SPRINT) zeigt für die Nierenpatient/innen bei niedrigerem systolischen Ziel-BD $(<120 \mathrm{~mm} \mathrm{Hg})$ weniger kardiovaskuläre Ereignisse als bei den Patient/ innen mit systolischem Ziel-BD $<140 \mathrm{~mm} \mathrm{Hg}$, allerdings um den Preis einer signifikanten, im Mittel um $0,15 \mathrm{ml} / \mathrm{min} / 1,73 \mathrm{~m}^{2}$ pro Jahr höheren Rate an eGFR-Verlust [403]. Letzteres darf als Hinweis genommen werden, dass $\mathrm{zu}$ niedrige Zielwerte nicht günstig sind und es ist mit etwas vermehrt unerwünschten Ereignissen zu rechnen. Auf Basis einer zwar retrospektiven, aber sehr großen Kohorte von 389.419 behandelten Hypertoniker/innen des Kaiser Permanente Southern California Health System ergibt sich nicht ein singulärer Zielwert, sondern ein möglicher Zielkorridor von systolisch 130-139 mm Hg und diastolisch 60-79 mm Hg [404]. Dieser Korridor ergibt sich nach der Analyse des zusammengesetzten Endpunktes Mortalität und Erreichen einer ESRD. Für ESRD allein lag der optimale systolische BD (adjusted hazard ratio $\leq 1$ ) sogar bei $110-139 \mathrm{~mm} \mathrm{Hg}$, nicht jedoch bei Patient/innen mit zusätzlich Diabetes, für die wieder der engere Zielbereich von 130-139 mm Hg zutraf [404]. Auch die SPRINT-Daten sprechen wegen des eGFR-Verlustes gegen ein Ziel $<120 \mathrm{~mm}$ Hg systolisch [403]. Eine weitere Analyse der gleichen Autor/ innen ergab, dass bei schwerer, das heißt resistenter Hypertonie im Vergleich zu nicht-resistenten Hypertoniker/innen das Risiko für ESRD um 32\% erhöht war [405]. Eine ältere Meta-Analyse von 11 Studien und 1860 nicht-diabetischen CKD-Patient/innen ergab, dass bei systolischem BD von $110-129 \mathrm{~mm} \mathrm{Hg}$ und einer Proteinurie von weniger als $2 \mathrm{~g} / \mathrm{Tag}$ das geringste Risiko einer Progression der Nierenerkrankung bestand [406]. Der Einsatz von ACE-Hemmern erwies sich ebenfalls als vorteilhaft.

Aus den genannten Daten ergibt sich, dass es schwierig ist je einen Schwellenwert für systolischen und diastolischen BD festzulegen. Dazu kommt, dass Faktoren wie Alter, Begleiterkrankungen wie Diabetes, Herzinsuffizienz oder andere kardiovaskuläre Erkrankungen, subjektive Verträglichkeit der Therapie, Wirkung der Antihypertensiva auf die individuelle Nieren- 
Tab. 28 Zielwerte der Blutdruckeinstellung für Hypertoniker/innen mit Nierenerkrankungen

\begin{tabular}{|l|l|l}
\hline Bei Niereninsuffizienz: & Systolisch $\mathrm{mm} \mathrm{Hg}$ & \multicolumn{1}{c}{ Diastolisch $\mathrm{mm} \mathrm{Hg}$} \\
\hline Office-Blutdruck (abhängig von Individualfaktoren - siehe Text) & $<130$ (evtl. <139) & $<80$ \\
\hline & Nicht unter 110 & Nicht unter 70 \\
\hline Bei Dialyse: & & \\
\hline Prädialyse-0ffice-Messung & $138-166$ & - \\
\hline Messung zuhause am Dialyse-freien Tag & $<145$ & - \\
\hline Nach Nierentransplantation: & $\leq 140$ & - \\
\hline & Ev. wie bei Niereninsuffizienz & Ev. wie bei Niereninsuffizienz \\
\hline
\end{tabular}

funktion oder Elektrolyt-Entgleisungen ebenfalls zu berücksichtigen sind. Somit sind Therapieziele individuell anzupassen, weshalb für Hypertoniker/innen mit Nierenerkrankungen und Niereninsuffizienz Stadium G1-G5 ein BD-Ziel von systolisch < $130 \mathrm{~mm} \mathrm{Hg}$, bei manchen Patient/innen (siehe Individualfaktoren) $<139 \mathrm{~mm} \mathrm{Hg}$ und nicht unter $110 \mathrm{~mm}$ Hg und diastolisch $<80 \mathrm{~mm} \mathrm{Hg}$, nicht aber unter $70 \mathrm{~mm} \mathrm{Hg}$, gilt. Eine Zusammenfassung der Zielwerte gibt Tab. 28.

\section{Hypertonie und Dialyse}

Die BD-Regulation bei Dialysepatient/innen ist komplex und dementsprechend schwierig ist es, Empfehlungen für BD-Zielwerte abzugeben. Es beginnt schon mit der Schwierigkeit, welche Messzeitpunkte - vor oder nach Dialyse, an den Dialyse-freien Tagen oder 24- bis $48 \mathrm{~h}$-Durchschnittswerte herangezogen werden sollen, um danach therapeutische Entscheidungen zu treffen. Es gibt auch Hinweise (allerdings nur aus Beobachtungsstudien), dass im Verlauf der Jahre an Dialyse sich mögliche günstige BD-Zielwerte verändern, was unter anderem auf eine sich ändernde Gefäßsteifigkeit zurückzuführen ist. Eine Studie an 56.338 inzidenten und 69.590 prävalenten Dialysepatient/innen ergab, dass ein prädialytischer systolischer BD $<120$ mm Hg mit der höchsten Mortalität im ersten Jahr assoziiert war, während prädialytische Werte von 160-180 mm Hg die geringste Mortalität aufwiesen [407]. Im späteren Verlauf waren eher niedrigere Werte die hinsichtlich Mortalität günstigsten. Rezent wurde mit ambulanten 24-Stunden-Blutdruckmessungen gezeigt, dass eine U-förmige Kurve sowohl für systolischen $\mathrm{BD}$ wie Pulsdruck besteht, wobei das Vorhandensein von Herzinsuffizienz oder Vorhofflimmern zu einem konträren Verhalten im Vergleich zu Patienten ohne Herzinsuffizienz oder Vorhofflimmern führt [408]. Im Detail fanden die Autoren, dass Patient/innen mit Vorhofflimmern und/oder Herzinsuffizienz bei niedrigen Pulsdruck-Werten das höhere Risiko für Sterblichkeit jedweder wie kardiovaskulärer Ursache aufwiesen. Im Gegensatz dazu zeigte sich bei Patient/innen ohne Vorhofflimmern oder Herzinsuffizienz, dass mit steigendem Pulsdruck das Risiko für Sterblichkeit zunahm [408].
Für die Tolerabilität einer Dialysebehandlung ist die Vermeidung von BD-Entgleisungen, vor allem nach unten, gelegentlich aber auch exzessiv nach oben, ein klinisch wichtiges Ziel. Daneben gilt es natürlich auch die kardiovaskulären Risiken für dieses Kollektiv so weit möglich zu reduzieren. Nicht-traditionelle Risikofaktoren wie Anämie, Urämietoxine, Hyperparathyreoidismus und natürlich die Volumsbelastung durch den arteriovenösen Dialyseshunt rücken in den Vordergrund und sind möglicherweise bestimmender als der Hypertonus. Das Muster kardiovaskulärer Ereignisse von Dialysepatient/innen differiert von dem anderer Patient/innengruppen. Eine Studie zu Magnetresonanz-Untersuchungen des Herzens von 134 Dialysepatient/innen ergab, dass 28,4\% der Patient/innen Zeichen einer Myokardfibrose aufwiesen, was bei nierengesunden Hypertoniker/innen nicht typisch wäre [409]. Die Behandlung typischer Risikofaktoren wie Hyper- und Dyslipidämie bewirkt bei Dialysepatient/innen keine Risikoreduktion [410]. Die schon früh bei CKD-Patient/innen bestehende erhöhte Gefäßsteifigkeit [411] und die gleichzeitig bestehende Mikrozirkulationsstörung erhöhen das Risiko und können bei Blutdruckabfall zu kardialer wie zerebraler Perfusionsminderung mit konsekutiven Langzeitfolgen führen.

Grundsätzlich kommen alle Wirkstoffklassen von Antihypertensiva zum Einsatz, jedoch ist festzuhalten, dass Patient/innen mit höhergradiger Niereninsuffizienz aus vielen Antihypertensiva-Studien ausgeschlossen waren, kaum Studien bei diesem Kollektiv existieren und direkte Vergleichsstudien selten sind. Im Hypertension in Hemodialysis Patients Treated with Atenolol or Lisinopril (HDPAL) Trial wurden Lisinopril und Atenolol bei Dialysepatient/innen mit Hypertonie und linksventrikulärer Hypertrophie direkt verglichen [412]. Die Linksherzhypertrophie besserte sich unter beiden Medikamenten. Allerdings kam es in der Lisinopril-Gruppe zu deutlich mehr Hospitalisierungen wegen Herzinsuffizienz, sodass die Studie vorzeitig beendet wurde. BD-Selbstmessungen ergaben niedrigere Werte unter Atenolol, obwohl die Lisinopril-Gruppe deutlich mehr zusätzliche Antihypertensiva einnahm und das Zielgewicht bei Dialyseende um nahezu $3 \mathrm{~kg}$ im Studienverlauf abgesenkt wurde 
[412]. Bei Atenolol genügt es, dreimal wöchentlich eine Dosis nach der Dialyse zu verabreichen.

Die Definition von Zielwerten reduziert sich nach einer Meta-Analyse von Heerspink et al. auf die Empfehlung, dass routinemäßig eine Behandlung zur BDSenkung bei Dialysepatient/innen erwogen werden soll, um die hohe kardiovaskuläre Morbidität und Mortalität zu reduzieren [413]. Bei 1679 Patient/innen aus 8 Studien führte die Senkung des BDs systolisch um 4,5 und diastolisch 2,3 $\mathrm{mm} \mathrm{Hg}$ durch aktive Therapie zu einer Senkung des relativen Risikos für kardiovaskuläre Ereignisse auf 0,71, der Mortalität jedweder Ursache auf 0,80 und der kardiovaskulären Mortalität auf 0,71 im Vergleich zu Kontrollen [413]. Mögliche Zielwerte ergeben sich vielleicht aus einer Analyse der Chronic Renal Insufficiency Cohort (CRIC) Studie. Bansal et al. untersuchten bei 403 Patient/innen mit Hämodialyse im Median 6 Monate nach Dialysebeginn den Zusammenhang systolischer $\mathrm{BD}$ an der Dialyse und Mortalität jedweder Ursache und bei 326 davon auch den Zusammenhang zwischen zuhause gemessenen systolischen Blutdruckwerten und Mortalität [414]. Die Autoren zeigen hinsichtlich peri-dialytischem systolischen BD einen signifikanten und nicht-linearen Zusammenhang mit der Mortalität. Verglichen mit einem systolischen BD von 151-166 mm $\mathrm{Hg}$ als Referenz war sowohl mit niedrigerem (<138 mm Hg; hazard ratio 2,49) als auch mit höherem (>166 mmHg; hazard ratio 2,52$) \mathrm{BD}$ die Mortalität signifikant erhöht. Betreffend systolischen BD zwischen zwei Dialysen zuhause zeigte sich eine lineare Korrelation mit systolischen Werten $<113 \mathrm{~mm}$ Hg als Referenz und erhöhter Mortalität mit höheren Werten ( $>145 \mathrm{~mm} \mathrm{Hg}$; hazard ratio 3,35). Die genannten Berechnungen ergaben sich nach Adjustierung für Alter, Geschlecht, Rasse, Rauchen, body mass index, Diabetes und kardiovaskuläre Vorgeschichte [415]. Aus dieser Analyse ergibt sich die Empfehlung, bei Berücksichtigung der an der Dialyse zu Beginn gemessenen Werte, als systolischen Zielkorridor 138-166 mm Hg anzustreben, hinsichtlich der zuhause gemessenen Werte ein Ziel von $<145 \mathrm{~mm} \mathrm{Hg}$ anzupeilen, zumal diese Bereiche mit der jeweils geringsten Mortalität verknüpft sind.

Nicht vergessen werden soll als „antihypertensive Therapie“ bei Dialysepatient/innen die Volumenkontrolle durch Dialyse mit Einstellung des richtigen Zielgewichtes nach Dialyse sowie die ausreichend lange Dialyse, zumal auch Urämie blutdrucksteigernd wirken kann.

\section{Hypertonie nach Nierentransplantation}

Wie auch an der Dialyse gibt es für Hypertonie nach Nierentransplantation keine randomisierten Studien, wie der Bluthochdruck am besten zu managen ist [412]. Nach Nierentransplantation kommen multiple, Hypertonie-begünstigende Faktoren zusammen. Es können seitens des Organempfängers/der
Organempfängerin eine vorbestehende Hypertonie, Linksherzhypertrophie, weiter Faktoren seitens des Organspenders/der Organspenderin wie präexistente Hypertonie, Alter oder Gefäßprobleme, dann chirurgische Probleme wie eine arterielle Anastomosenstenose, CMV-Infektion, mögliche akute oder chronische Abstoßung, Rekurrenz der Nieren-Grunderkrankung im Transplantat, de novo Nierenerkrankung im Transplantat und auch Nebenwirkungen der Immunsuppressiva, insbesondere CalcineurinInhibitoren, Glucocorticoide eine Rolle spielen [415]. Hypertonie betrifft etwa dreiviertel aller Nierentransplantierten und wieder ergibt sich die Problematik, welche Messmethode optimal ist. Eine Untersuchung an 120 Transplantierten kommt zum Schluss, dass die Methode, die in der SPRINT-Studie angewendet wurde, geeignet ist, den Weißkittel-Effekt zu vermeiden [416]. In der praktischen Erfahrung spielt dies selbst bei Patient/innen mit langjährig ausgezeichneter Transplantatfunktion immer noch eine Rolle. Eine italienische Studiengruppe stellte die Korrelation von Hypertonie zu einem Transplantat-Funktionsverlust her [417]. Ein Anstieg des systolischen BDs tagsüber um $5 \mathrm{~mm}$ Hg erhöhte das Risiko, den kombinierten Endpunkt aus GFR-Verlust $>30 \%$, Erreichen der Dialysepflichtigkeit oder Tod zu erreichen um $11 \%$, beim nächtlichen Blutdruck war das Ergebnis mit $10 \%$ je $5 \mathrm{~mm} \mathrm{Hg}$ ebenfalls signifikant. Es bestand eine inverse Relation zwischen BD-Anstieg und GFR-Abfall [417]. Daten von 24.404 Empfängern eines ersten Nierentransplantates aus der Collaborative Transplant Study Datenbank ergaben, dass Betroffenen mit systolischem BD >140 mm Hg nach einem Jahr, jedoch BDKontrolle auf Werte unter $140 \mathrm{~mm} \mathrm{Hg} 3$ Jahre nach Transplantation ein signifikant besseres TransplantatÜberleben aufwiesen (relatives Risiko 0,79; $p<0,001$ ) als Transplantierte mit persistierender Hypertonie [418]. Gelang es weiter, 3 Jahre posttransplantationem den BD zum Jahr 5 hin zu senken, so bewirkte dies ein signifikant besseres 10-Jahres-Transplantatüberleben (relatives Risiko 0,83; $p=0,01$ ) während ein (auch nur temporärer) Anstieg des systolischen BDs nach 3 Jahren $>140 \mathrm{~mm}$ Hg mit einem schlechteren Transplantatüberleben einherging (relatives Risiko $1,37 ; p<0,001)$. Auch bei Lebendspende konnte nachgewiesen werden, dass eine signifikante Assoziation zwischen höherem BD und Transplantat-Versagen, sogar unabhängig von der jeweiligen Transplantatfunktion zu Beginn, besteht [419].

Für die Frage, welche Antihypertensiva nach Nierentransplantation zum Einsatz kommen sollen, gibt es wohl etliche, meist relativ kleine Untersuchungen, jedoch keine harten Daten. Eine Meta-Analyse von 60 Studien mit insgesamt 3802 Patient/innen nahm Kalziumantagonisten und ACE-Hemmer in den Fokus [420]. Verglichen wurden Kalziumantagonisten mit Placebo oder ohne Therapie, ACE-Hemmer mit Placebo oder ohne Antihypertensiva und Kalziumantagnisten mit ACE-Hemmer. Eine Therapie mit 
Kalziumantagonisten führte $\mathrm{zu}$ geringerem Transplantat-Verlust (risk ratio 0,75 ) und zu einer besseren glomerulären Filtrationsrate um $4,5 \mathrm{ml} / \mathrm{min}$. Die entsprechende Auswertung hinsichtlich ACE-Hemmer war nicht konklusiv. Im Direktvergleich von Kalziumantagonisten mit ACE-Hemmern führten letztere $\mathrm{zu}$ einer Abnahme der glomerulären Filtrationsrate im Mittel um 11,5 ml/min, allerdings auch $\mathrm{zu}$ weniger Proteinurie um den Preis einer erhöhten Rate an Hyperkaliämie (relatives Risiko 3,7). Die Analyse hinsichtlich Transplantatverlust war inkonklusiv. Die Autor/innen empfehlen Kalziumantagonisten als Erstlinientherapie für Nierentransplantierte [420]. Eine große österreichische Untersuchung (retrospektive offene Kohortenstudie mit Propensity Score Analyse) von 2031 Transplantierten, die mindestens 3 Monate überlebten, zeigte, dass das 10-Jahres-Überleben von Patient/innen mit ACE-Hemmer/ARB Therapie mit $74 \%$ deutlich über dem von Patient/innen ohne ACE-Hemmer/ARB Therapie lag, bei denen es lediglich $53 \%$ betrug [421]. Die hazard ratio für Gebrauch von ACE-Hemmer/ARBs hinsichtlich Mortalität lag bei 0,57. Das 10-Jahres-Transplantat-Überleben war unter ACE-Hemmer/ARB Therapie mit $59 \%$ gegenüber $41 \%$ bei Patient/innen ohne ACE-Hemmer/ARB Therapie ebenfalls überlegen. Aufgrund ihrer Analyse empfehlen die Autor/innen den häufigeren Einsatz von ACE-Hemmern oder ARB bei Patient/innen nach Transplantation [421]. Eine ganz rezente Meta-Analyse fand 71 randomisierte kontrollierte Studien und bestätigte, dass Kalziumkanal-Blocker das Risiko für Transplantatverlust reduzierten (risk ratio 0,58; $95 \%$ Konfidenzintervall 0,38-0,89), die GFR verbesserten und den BD senkten [422]. ACE-Hemmer reduzierten ebenfalls das Risiko für Transplantatverlust (risk ratio 0,62; $95 \%$ Konfidenzintervall 0,40-0,96) aber verminderten die Nierenfunktion und erhöhten das Risiko für Hyperkaliämie. Im Direktvergleich von Kalziumkanal-Blocker und Blockern des Renin-AngiotensinSystems verbesserten erstere die GFR im Mittel um $11 \mathrm{ml} / \mathrm{min}$ und verminderten die Kaliumspiegel, die BD-Senkung war vergleichbar [422]. Die Autoren leiten daraus die Empfehlung von Kalziumkanal-Blockern als Ersttherapie ab. Ein weiterer Aspekt ist, dass Calcineurin-Inhibitoren wie sie als Immunsuppressivum fast standardmäßig zum Einsatz kommen über eine Vasokonstriktion der afferenten Arteriolen den Posttransplant-Hypertonus begünstigen. Diese Wirkung wird durch die Vasodilatation von DHP-Kalziumantagonisten ebendort wieder aufgehoben [415]. Diese Annahme wird noch durch einen Direktvergleich Nifedipin mit Lisinopril erhärtet, wo in einer kleinen Kohorte von initial 154 Patient/innen mit Nierentransplantation insgesamt 123 Patient/innen über ein Jahr und 64 Patient/innen über zwei Jahre in doppelt-blindem Studiendesign $30 \mathrm{mg}$ Nifedipin oder $10 \mathrm{mg}$ Lisinopril einmal täglich erhielten [423]. Waren beide Substanzen hinsichtlich Blutdrucksenkung gleichwertig und sicher wirksam, so wies die Nife-
dipin-Gruppe nach einem Jahr eine um 9,6 $\mathrm{ml} / \mathrm{min}$ und nach zwei Jahren 10,3 ml/min signifikant höhere glomeruläre Filtrationsrate auf als die Lisinoprilbehandelten [423]. Natürlich kann der Unterschied auf den bekannten hämodynamischen Effekt der ACEHemmer/ARB Therapie auf die eGFR zurückzuführen sein. In der Praxis ist es ohnedies häufig erforderlich mehr als ein Antihypertensivum einzusetzen, sodass eine Kombination beider Substanzgruppen nicht selten zum Einsatz kommt.

Hinsichtlich Zielwerte der Blutdruckeinstellung ist wohl die Untersuchung der Collaborative Transplant Study als geeignet heranzuziehen [418]. Eine Einstellung auf systolische BD-Werte von konstant $\leq 140 \mathrm{~mm}$ Hg war mit dem besten Langzeit-Transplantatüberleben assoziiert. Initial, das heißt in den ersten Wochen nach Transplantation, sind im Hinblick auf ausreichende Organperfusion und Vermeidung einer Thrombose im Transplantat Werte bis 160/90 mm Hg durchaus tolerierbar. Später gelten aber die gleichen Ziele der Vermeidung von Hypertonie-vermittelten Organschäden wie für Nicht-Transplantierte. Dass die gleichen BD-Zielwerte wie bei Niereninsuffizienten Stadium G1-G4 bei Transplantierten herangezogen werden kann, läge zwar nahe, ist aber durch entsprechende Studien nicht belegt.

\section{Ausblick}

Im Hinblick auf die optimale Behandlung des Bluthochdrucks bei CKD bleiben noch viele Fragen offen, nicht zuletzt auch die nach Zielwerten (siehe Tab. 27). Renale Endpunkte werden in Hypertonie-Studien häufig nur als sekundäre Endpunkte analysiert. Die Definition der renalen Endpunkte, z. B. das Ausmaß der eGFR-Abnahme, variiert und erschwert somit Meta-Analysen. Vielleicht wird hier eine neue Initiative zur einheitlichen Definition renaler Endpunkte eine Verbesserung bringen. Major Adverse Renal Events - MARE, mit exakt definierten Auswertekriterien für die Komponenten dieses zusammengesetzten Endpunktes könnte vielleicht ein neuer Standard für Studien in der Nephrologie werden [424]. Speziell bei CKD-Patient/innen mit Hypertonie sind die Auswirkungen verschiedener Maßnahmen zur BDNormalisierung leider unzureichend untersucht. Dies gilt sowohl für den optimalen Mess-Zeitpunkt, die Zielbereiche in Abhängigkeit vom jeweiligen Stadium der Niereninsuffizienz wie auch die Berücksichtigung vieler individuell Patient/innen-bezogener Faktoren (Alter, Geschlecht, Komorbidität usw.). Ein systematischer Review von Leitlinien für die pharmakologische Therapie chronischer Erkrankungen in der medizinischen Erstversorgung zeigt eigentlich erschreckende Ergebnisse [425]. Von 421 klinischen Praxis-Leitlinien konnten lediglich $23,5 \%$ als qualitativ hochwertig eingestuft werden. Auch wenn gut anwendbare Leitoder Richtlinien natürlich auf vielen verschiedenen Faktoren wie Klarheit der Präsentation, des Umfangs 
und der Ziele fußen, so ist wesentlich, ob eine brauchbare Datenlage als Grundlage von Empfehlungen zur Verfügung steht. Hinsichtlich Hypertonie und chronisch renale Insuffizienz bleibt noch viel zu tun, insbesondere wenn man bedenkt, dass beispielsweise adoleszente Hypertoniker/innen ein 5-fach erhöhtes Risiko aufweisen, 20 Jahre später ein Dialyse-pflichtiges Nierenversagen zu erleiden [426].

\section{Fact box Blutdruck und Nierenerkrankungen}

- Hypertonie kann die Ursache für eine Nierenschädigung darstellen.

- Bei $80 \%$ aller Patient/innen mit Nierenerkrankungen kommt es zum Auftreten einer Hypertonie.

- Zur Diagnosestellung hat die ambulante 24-h-Blutdruckmessung bei Patient/innen mit chronischer Niereninsuffizienz die höchste Wertigkeit. Möglicherweise wird in Zukunft die unbeobachtete automatisierte oszillometrische Ordinations-Blutdruckmessung einen ähnlichen Stellenwert bekommen, ist aber gegenwärtig bei CKD-Patient/innen nicht validiert. Die Blutdruck-Selbstmessung hat insbesondere in der Therapieüberwachung einen Stellenwert.

- Die hypertensive Nephropathie ist nach der diabetischen Nierenerkrankung die zweithäufigste Ursache für dialysepflichtiges Nierenversagen, was die Wichtigkeit einer guten Blutdruckeinstellung zur Verhinderung der Nierenschädigung unterstreicht.

- Auch wenn bei CKD nur unzureichend untersucht, stellen anhaltende Lebensstil-Modifikationen mit Bemühung um Gewichtsnormalisierung, Ernährung reich an Frischobst und -gemüse, Bewegung, Beenden des Rauchens die Basis jeder antihypertensiven Therapie dar.

- Trotz gewisser Unsicherheiten ist eine Verminderung des Salzkonsums, z.B. in Fertignahrung enthalten, auch für Patient/innen mit CKD sinnvoll und protektiv und reduziert kardiovaskuläre und renale Ereignisse.

- Kenntnisse über Pharmakokinetik und -dynamik sind bei der Dosierung von Antihypertensiva mit renaler Elimination zu berücksichtigen.

- Die Erstlinientherapie soll mit ACE-Hemmern oder ARBs erfolgen, gefolgt von und/oder kombiniert mit Kalziumkanal-Blockern und/oder lang wirksamen Diuretika.

- Andere Substanzklassen wie Betablocker, zentrale Sympatholytika, Alphablocker, Mineralocorticoid-Rezeptoren-Blocker und direkte Vasodilatatoren haben als Kombinationspartner insbesondere hinsichtlich einer individualisierten antihypertensiven Therapie ebenfalls einen Stellenwert.

- Therapieziele sind in Abhängigkeit von individuellen Faktoren wie Alter, Ausmaß der Niereninsuffizienz, Ausmaß der Proteinurie, Begleiterkrankungen, subjektiver Tolerabilität, Elektrolytentgleisungen usw. festzulegen und sollten systolisch bei <130 (ev. <139; nicht unter 110) $\mathrm{mm} \mathrm{Hg}$ und diastolisch $<80$ (nicht unter 70) $\mathrm{mm} \mathrm{Hg}$ liegen (Office-Blutdruck).
- Bei Dialysepatient/innen mit Hypertonie ist gesichert, dass eine Blutdrucksenkung sinnvoll ist. Wird der prädialytische Office-Blutdruck herangezogen, ist ein Zielkorridor für den systolischen Blutdruck von 138-166 mm Hg anzustreben. Nimmt man Werte vom Dialyse-freien Tag zuhause, so sollte der systolische Wert $<145 \mathrm{~mm} \mathrm{Hg}$ (Selbstmessung) sein.

- Für Patient/innen mit Hypertonie nach Nierentransplantation sollen bevorzugt Dihydropyridin-Kalziumantagonisten und ACE-Hemmer/ARBs zum Einsatz kommen. Ziel ist ein systolischer Blutdruck konstant $\leq 140 \mathrm{~mm} \mathrm{Hg}$ (Office-Blutdruck).

\section{Blutdruck und neurologische Erkrankungen}

Arterielle Hypertonie ist der Hauptrisikofaktor für zerebrovaskuläre Erkrankungen [427, 428] und eine optimale antihypertensive Therapie ist sowohl im Sinne einer primären als auch sekundären Prävention erforderlich. In der Akut-Situation ist hinsichtlich der Behandlung zwischen ischämischem Schlaganfall und intrakranieller Blutung zu differenzieren. Als eigener Punkt wird außerdem die Prävention von vaskulärer Demenz diskutiert.

\section{Akuter ischämischer Schlaganfall}

Arterielle Hypertonie wird als wichtigster Risikofaktor für Schlaganfall eingeschätzt und es werden bis zu 2/3 aller Schlaganfälle auf einen erhöhten BD zurückgeführt. Ca. $25 \%$ aller hypertensiven Notfälle mit Endorganschaden sind zerebrovaskulärer Genese, wie in einem oberitalienischen Kollektiv untersucht wurde [429]. Bis zum Jahr 2000 galt deshalb der Konsens, dass eine intensive BD-Senkung auch im Rahmen des akuten Schlaganfalls durchgeführt werden sollte. Über die letzten Jahre zeigte sich jedoch zunehmend, dass mit dieser Strategie zum Teil schlechtere Ergebnisse erzielt wurden [430]. Dieses Phänomen kann durch eine Verminderung der Perfusion an den Grenzbereichen der Ischämie der sogenannten Penumbra erklärt werden [431]. In der akuten Phase des Schlaganfalls zeigen bis zu 75\% der Patient/innen deutlich erhöhte Blutdruckwerte, obwohl nur ca. die Hälfte der Patient/innen vor dem Ereignis eine diagnostizierte arterielle Hypertonie aufweisen. In den meisten Fällen kommt es auch zu einem Absinken des BDs zum Teil bereits 90 min nach dem Akutereignis [432]. Es ist dabei sehr wahrscheinlich, dass eine ausgeprägte arterielle Hypertension jenseits der Grenze der autoregulativen Kapazität der Hirngefäße (aufgrund des Hirnödems, des hämorrhagischen Umbaus im Rahmen des ischämischen Schlaganfalls, als auch durch kardiale und renale Komplikationen), aber auch eine extreme Hypotension (durch Verminderung der Durchblutung im ischämischen Hirnbereich) zu neurologischen Symptomen führt, besonders da die Autoregulation im Ischämiegebiet aufgehoben ist. Aus diesem Grund ist - theoretisch - ein optimaler Blutdruck- 
korridor im Rahmen des Akutereignisses anzustreben. Ein solcher konnte bis heute nicht wissenschaftlich etabliert werden und ist auch aufgrund der unterschiedlichen Subtypen und Komorbiditäten nicht eindeutig zu definieren.

In randomisierten Studien zeigten sich keine unterschiedlichen funktionellen Ergebnisse auf Grund verschiedener Blutdruckzielwerte, wie eine Meta-Analyse ergab [433]. Eine Studie, welche bei Patient/innen in den ersten $48 \mathrm{~h}$ nach akutem ischämischen Schlaganfall eine bereits bestehende antihypertensive Therapie in einer Kohorte absetzte und in der anderen Kohorte fortführte, kam ebenso zu einem neutralen Ergebnis [434]. Als ein Resultat dieser Unsicherheit hat man sich in internationalen Richtlinien dazu entschlossen, keine Blutdrucksenkung innerhalb der ersten $24 \mathrm{~h}$ (teilweise auch innerhalb der ersten sieben Tage) nach einem ischämischen Schlaganfall durchzuführen, außer bei BD-Werten über 220/120 mm Hg [432] mit dem Zusatz, dass bei anderen medizinischen Indikationen für eine BD-Senkung diese natürlich sehr wohl durchgeführt werden sollte. Auch in diesem Fall sollte die BD-Senkung langsam um $15 \%$ in den ersten $24 \mathrm{~h}$ erfolgen [432].

Das primäre Ziel bei Patient/innen mit der Verdachtsdiagnose auf ein akutes zerebrovaskuläres Ereignis ist eine rasche neuroradiologische Untersuchung (zerebrale Computertomographie oder Magnetresonanztomographie), um zwischen ischämischem Schlaganfall oder intrazerebraler Blutung $\mathrm{zu}$ unterscheiden. Sind die Kriterien zur systemischen Thrombolyse im Rahmen eines diagnostizierten ischämischen Schlaganfalls gegeben, soll der BD unter 185/110 mm Hg gesenkt werden, wie in der $\mathrm{Zu}$ lassungsstudie für Alteplase beschrieben wurde, um das Risiko einer hämorrhagischen Transformation zu minimieren [432, 435]. In einer großen Observationsstudie bei Patient/innen nach Thrombolyse zeigte sich, dass ein erhöhter BD in den ersten $24 \mathrm{~h}$ linear mit dem Auftreten einer intrazerebralen Blutung vergesellschaftet war. In der gleichen Patient/ innengruppe konnte ein U-förmiger Zusammenhang zwischen BD und Tod innerhalb der ersten $24 \mathrm{~h}$ oder Betreuungsnotwendigkeit zum Zeitpunkt von drei Monaten festgestellt werden. Die besten Ergebnisse wurden bei Patient/innen mit Blutdruckwerten zwischen 141-150 mm Hg systolisch gesehen [436]. In der rezenten ENCHANTED Studie wurden $2227 \mathrm{~Pa}-$ tient/innen mit ischämischem Insult, bei denen eine Thrombolyse durchgeführt wurde, zu einem konventionellen Blutdruckziel (<180 mm Hg) und einem intensiveren Blutdruckziel (130-140 mm Hg, innerhalb einer Stunde zu erreichen) randomisiert [437]. Die erreichten Blutdruckunterschiede in den ersten $24 \mathrm{~h}$ waren relativ gering (144,3 versus $149,8 \mathrm{~mm} \mathrm{Hg}$ ). Das funktionelle Outcome nach 3 Monaten (Rankin Skala) war nicht unterschiedlich, in der intensiver therapierten Gruppe traten signifikant weniger in- trakranielle Blutungen auf, die Anzahl an schweren Nebenwirkungen in beiden Gruppen war gleich.

Im Fall eines akuten Schlaganfalls sollte die Medikation bevorzugt intravenös gegeben werden, um Aspiration zu vermeiden. Aufgrund des niedrigen Nebenwirkungspotentials von Alpha-Rezeptorblockern (Urapidil) oder Betablockern (Labetolol) sind diese am häufigsten im Einsatz. Es sollte jedoch daran gedacht werden, dass im Falle von Komorbiditäten wie Myokardinfarkt oder Aortendissektion andere Blutdruckziele notwendig sind und entsprechend andere Medikamente verwendet werden können.

Der beste Zeitpunkt zum Wiederbeginn einer antihypertensiven Therapie (vor allem nach Pausieren aufgrund von Schluckbeschwerden) ist unklar, wobei ein Beginn nach $24 \mathrm{~h}$ beim symptomfreien Patient/ innen rational erscheinen. Es gibt keine klare Empfehlung, welche Substanzgruppe verwendet werden sollte, um den Zielblutdruck zu erreichen. Insbesondere in der CHHIPS [438] und SCAST [439]-Studie konnten Lisinopril bzw. Candesartan keine klaren Vorteile in der Behandlung aufzeigen.

\section{Intrazerebrale Blutung}

Eine spontane intrazerebrale Blutung geht mit hoher Mortalität und schwerer körperlicher Behinderung bei den Überlebenden einher. Erhöhter arterieller BD war in zahlreichen Studien mit einem Größenwachstum des Hämatoms verbunden, was als Hauptursache für das schlechtere Langzeitergebnis verglichen mit einem ischämischen Schlaganfall anzusehen ist [440]. Aus diesem Grund erscheint die Blutdrucksenkung eine vielversprechende Strategie, vor allem Bezug nehmend auf eine randomisierte Studie, welche zeigen konnte, dass die Reduktion des systolischen BDs unter $140 \mathrm{~mm} \mathrm{Hg}$ den zerebralen Blutfluss um das Hämatom nicht verschlechterte [441]. Die INTERACT-2-Studie [442], welche 2839 Patient/innen mit erhöhten systolischen Blutdruckwerten zu einer intensiven Blutdrucksenkung $(<140 \mathrm{~mm} \mathrm{Hg}$ innerhalb einer Stunde; erreicht wurden $150 \mathrm{~mm} \mathrm{Hg}$ ) versus einer konventionellen Therapie (systolisches Ziel unter $180 \mathrm{~mm} \mathrm{Hg}$; erreicht wurden $164 \mathrm{~mm} \mathrm{Hg}$ ) randomisierte, ergab, dass die intensive Blutdrucksenkung zwar nicht die Mortalität reduziert, jedoch das funktionelle Ergebnis drei Monate nach Ereignis verbesserte. Zusätzlich sollte darauf geachtet werden, dass die Blutdruckvariabiliät vor allem in den ersten $24 \mathrm{~h}$ nach einer intrakraniellen Blutung auf ein Minimum reduziert wird, um unerwünschte Ergebnisse zu vermeiden [443]. Diese positiven Ergebnisse konnten jedoch in einer zweiten großen Studie nicht repliziert werden, welche 1000 Patient/innen mit akuter intrazerebraler Blutung zu systolischen Zielblutdruckwerten 110-139 mm Hg (erreicht wurden in den ersten $2 \mathrm{~h} 129 \mathrm{~mm} \mathrm{Hg}$ ) verglichen mit 140-170 mm Hg (erreicht wurden in den ersten $2 \mathrm{~h} 141 \mathrm{~mm} \mathrm{Hg}$ ), die in den ersten $4,5 \mathrm{~h}$ erreicht werden sollten, untersuch- 
te und keinen Vorteile für eine der beiden Gruppen erbrachte [444]. Es kam allerdings zu vermehrten renalen Ereignissen in der intensiver behandelten Gruppe. Dennoch wird von den meisten neurologischen Fachgesellschaften bei Patient/innen mit akuter intrazerebraler Blutung und erhöhtem systolischen Blutdruckwerten (150-220 mm Hg) eine Blutdrucksenkung unter $140 \mathrm{~mm} \mathrm{Hg}$ systolisch als sicher und möglicherweise auch mit einem besseren funktionellen Erholung assoziiert angesehen. Eine Metaanalyse basierend auf individuellen Patient/innendaten dieser beiden Studien zeigte, dass grundsätzlich eine Senkung des systolischen BDs mit einem besseren funktionellen Ergebnis nach 3 Monaten assoziiert ist. Hierbei waren die Effekte mit stärkerer BD-Senkung deutlicher. Eine hohe BD-Variabilität innerhalb der ersten $24 \mathrm{~h}$ war mit einer erhöhten Komplikationsrate verbunden. Patient/innen, bei denen der systolische BD um mehr als $60 \mathrm{~mm} \mathrm{Hg}$ innerhalb der ersten Stunde gesenkt wurde, hatten tendenziell ein schlechteres Outcome nach 3 Monaten (Moullaali et al., präsentiert European Stroke Conference 2019 Milano, Italy).

\section{Primärprävention}

Epidemiologisch zeigt sich ein direkter linearer $\mathrm{Zu}$ sammenhang zwischen dem Risiko für einen Schlaganfall (ischämisch oder hämorrhagisch) und ansteigenden BD-Werten bereits ab einem Wert von 115 mm Hg systolisch [5]. Bereits die Reduktion des arteriellen BDs um $10 \mathrm{~mm} \mathrm{Hg}$ systolisch und $5 \mathrm{~mm} \mathrm{Hg}$ diastolisch führt zu einer Risikoreduktion von bis $\mathrm{zu}$ $32 \%$ [11]. Studien haben zudem gezeigt, dass eine antihypertensive medikamentöse Therapie selbst bei Patient/innen mit prähypertensiven BD-Werten (120-139/80-89 mm Hg) das Risiko für einen Schlaganfall reduziert. Dies konnte in einer Meta-Analyse, welche 16 Studien mit über 70.000 Patient/innen einschloss, nachgewiesen werden [445]. Der Nutzen einer antihypertensiven Therapie zur Schlaganfallvermeidung ist auch bei Patient/innen jenseits des 80. Lebensjahres relevant [241]. Die Wahl der Medikamentenklasse zur Blutdrucksenkung zeigte wenig therapeutische Relevanz und sollte vor allem auf Komorbiditäten abgestimmt werden.

\section{Sekundäre Prävention}

Die Behandlung der arteriellen Hypertonie ist der wichtigste Faktor, um eine erfolgreiche sekundäre Prävention bei ischämischem Schlaganfall zu garantieren [446]. Das Risiko für einen zweiten Insult nach TIA oder Schlaganfall wird mit $4 \% / \mathrm{Jahr}$ geschätzt [447], wobei der zweite Schlaganfall meist klinisch schwerer verläuft. In der PROGRESS-Studie [448], welche 6105 Patient/innen mit Vorgeschichte einer TIA oder eines Schlaganfalls (ischämisch, als auch hämorrhagisch) einschloss, zeigte sich eine signifikante Risikoreduktion um 28\%, einen zweiten Schlaganfall zu erleiden, wenn die Patient/innen mit dem ACEHemmer Perindopril beziehungsweise noch besser in Kombination mit Indapamid (relative Risikoreduktion $43 \%$ ) behandelt wurden. In einer Post-Hoc-Analyse [449] zeigte sich der Behandlungseffekt verglichen mit einer Kombinationstherapie über alle Blutdruckwerte konstant: die relative Risikoreduktion betrug $47 \%$, $41 \%, 41 \%$ und $36 \%$ bei systolischen Ausgangsblutdruckwerten über 160, 140-159, 120-139 und unter $120 \mathrm{~mm} \mathrm{Hg}$. Bei antihypertensiver Monotherapie war die Blutdruckreduktion geringer ausgeprägt, und ein vorteilhafter Effekt konnte nur für systolische Blutdruckwerte über $140 \mathrm{~mm} \mathrm{Hg}$ nachgewiesen werden. Die jährlichen Schlaganfallereignisse waren direkt den erzielten systolischen Blutdruckwerten zu zuordnen, mit der niedrigsten Schlaganfallrate in der Quartile mit den niedrigsten systolischen Blutdruckwerten (median 112/72 mm Hg). Eine Meta-Analyse, welche 10 randomisierte Studien einbezog, bestätigte, dass die medikamentöse antihypertensive Therapie das Risiko eines weiteren Schlaganfalls um $32 \%$ reduzierte [450]. Im Gegensatz dazu konnte die sekundäre Observations-Analyse der PRoFESS-Studie zeigen, dass systolische Blutdruckwerte zwischen 130-139 mm Hg mit der niedrigsten Rekurrenz-Rate vergesellschaftet waren [451]. In einem Versuch, die optimalen Zielblutdruckwerte $\mathrm{zu}$ bestimmen, wurden in der SPS3-Studie [452] 3020 Patient/innen mit einem lakunären Schlaganfall auf systolische Blutdruckwerte unter 130 (127 wurden erreicht) verglichen mit unter 150 (erreicht wurden 138) $\mathrm{mm} \mathrm{Hg}$ randomisiert. Die niedrigeren Zielblutdruckwerte konnten einen neuerlichen Schlaganfall um 19\%, einen neuerlichen ischämischen Schlaganfall um $16 \%$ und einen hämorrhagischen Schlaganfall um $63 \%$ reduzieren. Es besteht der allgemeine Konsens, dass eine Reduktion des BDs unter $140 / 90 \mathrm{~mm} \mathrm{Hg}$ erreicht werden sollte, in Patient/innen mit einem rezenten lakunären Schlaganfall erscheinen systolische Blutdruckwerte unter $130 \mathrm{~mm} \mathrm{Hg}$ zielführend [446]. Ein früher oder initialer Beginn mit einer kombinierten antihypertensiven Therapie bei Hochrisikopatienten erscheint sinnvoll.

\section{Spezielle Situation: Hochgradige Stenosierung von} intra-, als auch extrakraniellen supraaortalen Arterien

Bei hochgradigen supraaortalen Stenosen, insbesondere wenn sie auch hämodynamisch wirksam sind, können Blutdrucksenkungen bei bereits oft ausgeschöpfter Vasomotorenreserve zu Infarkten im Grenzzonenbereich führen.

Es besteht weiterhin Unklarheit, ob die Zielblutdruckwerte in primärer als auch sekundärer Prävention des ischämischen Schlaganfalls ebenso für Patient/ innen mit intra- oder extrakraniellen hochgradigen Gefäßstenosen gültig sind, verglichen zu den Patient/ innen ohne diesen Gefäßveränderungen. Studien hinsichtlich der primären- und sekundären Prävention 
haben nicht systematisch Patient/innen mit hochgradiger Gefäßpathologie ausgeschlossen und keine randomisierte Studie hat sich direkt mit dieser Patient/ innenpopulation auseinandergesetzt. Eine einzelne retrospektive Untersuchung zweier großer Endarteriektomie-Studien [453] (NASCET und ECST) mit symptomatischer extrakranieller Stenose der Arteria carotis interna berichtet über einen zwar nur gering jedoch signifikant ausgeprägten Anstieg des Risikos für einen wiederholten Schlaganfall bei erhöhten Blutdruckwerten. Diese Aussage ist auch gültig für Patient/innen mit der Kombination einer hochgradigen Arteria carotis interna Stenose auf der einen und einem Verschluss auf der kontralateralen Seite. Im Gegensatz dazu zeigte sich in der Subgruppe der Patient/innen mit einer über $70 \%$ igen bilateralen Carotisstenose eine inverse Beziehung zwischen der Höhe des systolischen und diastolischen BDs und dem Schlaganfallrisiko: Patient/innen mit niedrigeren BD-Werten (unter $149 \mathrm{~mm} \mathrm{Hg}$ systolisch) wiesen ein höheres Schlaganfallrisiko auf (basierend auf 24 Ereignissen bei 337 Patient/innenjahren mit einer grenzwertigen Signifikanz von $P=0,04)$. Dieser Effekt war nach Carotis-Endarterektomie nicht mehr nachweisbar. In einer Observationsstudie bei Patient/ innen mit symptomatischer intrakranieller Gefäßstenose $(>50 \%)$ waren höhere BD-Werte assoziiert mit einem erhöhten Risiko eines wiederholt auftretenden ischämischen Schlaganfalles in der Nachbeobachtung über einen längeren Zeitraum [454]. Auch in Patient/innen mit hämodynamischen ausgelösten Infarkten aufgrund eines Carotisverschlusses zeigte sich mit niedrigeren BD-Werten (unter 130/85 mm Hg) ein geringeres Risiko für ein Wiederauftreten eines Schlaganfalls [455].

Zusammengefasst erscheint somit die Blutdrucksenkung in den meisten Patient/innen mit hochgradiger intra- oder extrakranieller Pathologie der arteriellen Blutgefäße sicher. Aufgrund klinischer Erfahrungen könnte in der Subgruppe der Patient/innen mit hochgradiger Gefäßpathologie und insuffizienter Kollateralversorgung sowie Wiederauftreten neurologischer Symptome bei normalen oder $\mathrm{zu}$ niedrigen Blutdruckwerten möglicherweise von geringgradig erhöhten BD-Werten profitieren.

\section{Prävention der vaskulären Demenz}

Die vaskuläre Demenz wird ganz wesentlich von einem nicht oder nicht ausreichend behandelten Bluthochdruck hervorgerufen. Bluthochdruck gilt als Hauptrisikofaktor der zerebralen Mikroangiopathie, die über Marklagerschädigungen (,white matter lesions“) zu vaskulärer Demenz führt. Auch bei der Alzheimer Demenz wird die Modifikation und Behandlung der arteriellen Hypertonie als Prognosegünstig diskutiert [456].

In der NUN-Studie [457] wurde beschrieben, dass die Grenzen zwischen neurodegenerativer und vas- kulärer Demenz fließend sind. Hinsichtlich der Prävention einer Demenz konnten mehrere Studien aufzeigen, dass die arterielle Hypertonie in der mittleren Lebensphase zu einer Reduktion der kognitiven Leistung sowohl bei vaskulärer als auch AlzheimerDemenz im späteren Leben führt [458-462]. In einem ausführlichen Review der American Heart Association (AHA) werden diese Ansichten unterstützt [72]. Das Komitee wies jedoch darauf hin, dass die Evidenz nicht ausreichend gegeben ist, dass antihypertensive Therapie die kognitive Leistung mittelfristig verbessert. Die SPRINT-MIND-Studie [463] untersuchte die Effekte einer intensiveren Blutdrucksenkung ( $<120 \mathrm{~mm} \mathrm{Hg}$; allerdings unbeobachtete automatische Office Messung) versus einer Standard-Blutdrucksenkung $(<140 \mathrm{~mm} \mathrm{Hg})$ auf das Auftreten von Demenz und „mild cognitive impairment“. Innerhalb einer Nachbeobachtungszeit von 5,1 Jahren kam es zu einer nicht-signifikanten Reduktion von Demenz um $17 \%$ und zu einer signifikanten Reduktion von „mild cognitive impairment“ um $19 \%$. Es wäre möglich, dass die Nachbeobachtungszeit von 5 Jahren zu kurz für stärkere Therapieeffekte ist, weiters waren die Studienteilnehmer/innen im Mittel schon 68 Jahre alt.

\section{Fact Box Blutdruckziele bei neurologischen Er- krankungen (Office-Blutdruck)}

\section{- Akuter ischämischer Schlaganfall:}

- <220/120 mm Hg außer bei Begleiterkrankungen (z.B. Lungenödem, kardiale Ischämie, bei denen entsprechend niedrigere Werte anzustreben sind) - <185/110 mm Hg bei Thrombolyse

- Intrazerebrale Blutung:

- <140 mm Hg systolisch bei Ausgangswerten zwischen 150 und $220 \mathrm{~mm}$ Hg systolisch (vermutlich auch bei höheren Ausgangswerten)

- Primärprävention:

- 130/70-79 mm Hg; positive Effekte bis zu 115/75 $\mathrm{mm} \mathrm{Hg}$ beschrieben

- Sekundärprävention:

- <140/90 mm Hg bei allen Patient/innen

Bevorzugte Medikamente: ACE-Hemmer/ARBs in Kombination mit CCB oder Thiaziddiuretikum

\section{Blutdruck während und nach der Schwanger- schaft}

Sowohl bei normotensiven wie bei den meisten hypertensiven Schwangeren sinkt der systolische Ordinations-BD im 2. Schwangerschaftsdrittel im Mittel um 6-15 mm Hg ab und kehrt im 3. Trimenon zu den Ausgangswerten zurück [464, 465]. Bei Frauen, die eine Präeklampsie entwickeln, sinkt der BD im 2. Trimenon weniger oder gar nicht ab und nimmt im 3. Trimenon stärker zu $[464,466]$. Bei bis zu $10 \%$ der Frauen ist während der Gravidität eine Hypertonie zu diagnostizieren. Sie ist eine Hauptursache für mütterli- 
che, fötale und neonatale Morbidität und Mortalität, vorrangig in jenen Fällen, in denen sich eine schwere Präeklampsie, eine Eklampsie oder ein HELLP Syndrom entwickelt [467, 468]. Folgende Formen werden unterschieden:

Chronische (präexistente) Hypertonie, also eine vorbestehende primäre oder sekundäre Hypertonie, betrifft 1-5\% der Schwangeren. Der Fötus ist bei Frauen mit chronischer Hypertonie mit und ohne Blutdruckbehandlung durch Wachstumsretardierung, intrauterinen Tod und - vorwiegend kardiale - Missbildungen gefährdet [469]. Bei der mütterlichen Mortalität führen hämorrhagische Insulte im Zusammenhang mit stark erhöhten systolischen Werten (>160 mm Hg), Präeklampsie und Thrombozytopenie [470, 471]. Disseminierte intravaskuläre Gerinnung und Organversagen sind die schwersten Komplikationen einer Präeklampsie [472].

Vorbestehende, chronische Hypertonie mit Präeklampsie, auch als aufgepfropfte Präeklampsie bezeichnet. Im ABDM bleibt in der Regel die Nachtabsenkung erhalten oder ist sogar verstärkt [473].

Schwangerschaftshypertonie. Sie ist wie die Präeklampsie Folge einer plazentaren Fehlentwicklung, tritt in der 2. Hälfte der Gravidität auf, in der Regel nach der 20. Woche und betrifft bis zu 5\% der Schwangeren. Ohne Progression zu Präeklampsie ist sie meist leicht und gutartig [472].

Schwangerschaftsinduzierte Hypertonie mit Präeklampsie. Im Gegensatz zur primären Hypertonie fand sich bei $45 \%$ der Frauen mit schwangerschaftsinduzierter Hypertonie im ABDM eine nächtliche Hypertonie, mit Präeklampsie waren es $80 \%$ [473].

Präeklampsie ist definiert als Hypertonie plus Proteinurie $>300-500 \mathrm{mg} / 24$-Stundenurin oder eine Protein-/Kreatinin-Ratio im Spontanharn $\geq 30 \mathrm{mg} /$ mmol (bzw. $\geq 300 \mathrm{mg} / \mathrm{g}$ ) nach der 20. Woche. Wenn nicht anders möglich, zählt auch eine in 2 Harnproben mit Teststreifen nachgewiesene Proteinurie von mindestens $2+[474,475]$. Präeklampsie tritt selten vor dem letzten Trimenon auf. Entwickelt sich eine Präeklampsie frühzeitig vor der 33. Schwangerschaftswoche, sind mütterliche und pränatale Komplikationen gehäuft, hingegen weist eine späte Entwicklung (nach der 36. Woche) einer zumeist leichten Präeklampsie eine gute Prognose auf [476]. Als Suchtest für Präeklampsie wird die Bestimmung der Ratio von sFlt-1 (soluble FMS-like tyrosine kinase-1; ein antiangiogenetischer Faktor)/PlGF (placental growth factor; ein angiogenetischer Faktor) empfohlen, die schon bei inzipienter Präeklampsie erhöht ist [477].

Die Ursache einer Präeklampsie wird primär in einer gestörten Entwicklung der Trophoblasten der Plazenta gesehen. Diese führt zu plazentarer Hypoxie und kann über eine Kaskade von Mediatoren bei der Mutter Hypovolämie, Oligohydramnion und vorzeitige Plazentalösung, generelle Vasokonstriktion und endotheliale Dysfunktion, oxydativen Stress, Inflammation und eingeschränkte Durchblutung aller
Organe sowie Aktivierung von Thrombozyten und plasmatischer Gerinnung verursachen, beim Fötus Wachstumsretardierung bei bis zu $30 \%$ [478, 479]. Präeklampsie ist eine während der Gravidität fortschreitende Multisystemerkrankung, die weder zuverlässig verhindert noch, sobald etabliert, rückgängig gemacht werden kann, außer durch Beendigung der Schwangerschaft. Nach der Entbindung bildet sie sich meist innerhalb weniger Tage, mitunter jedoch nur protrahiert, zurück.

Symptome und Befunde der Präeklampsie Geringe Ödeme sind in der Schwangerschaft häufig und unspezifisch. Hingegen sind ausgeprägte oder rasch zunehmende Ödeme, besonders in nicht abhängigen Körperpartien, verbunden mit rascher Gewichtszunahme infolge renaler $\mathrm{Na}$ - und Flüssigkeitsretention oder kapillären Lecksyndroms („capillary leak syndrome“), letzteres gekennzeichnet durch erhöhten Hämatokrit, hinweisend auf Präeklampsie.

Leichte Präeklampsie geht ohne Symptome und Organmanifestationen einher. Symptome wie Kopfschmerzen, Sehstörungen, epigastrische Schmerzen und Emesis weisen auf eine schwere Präeklampsie hin; typische Befunde sind Serumkreatinin $>1,1 \mathrm{mg} / \mathrm{dl}$, Proteinurie $>5 \mathrm{~g} / 24 \mathrm{~h}$, erhöhte Leberenzyme, Thrombozytopenie.

Ein hohes Risiko für eine Präeklampsie besteht bei hypertensiver Erkrankung während einer früheren Schwangerschaft, chronischen Nierenerkrankungen, Autoimmunerkrankungen wie SLE, Typ 1 oder 2 Diabetes und chronischem Bluthochdruck; ein mäßiggradiges Risiko für Präeklampsie besteht bei der ersten Schwangerschaft, im Alter von 40 Jahren oder darüber, bei einem $\mathrm{BMI}>35 \mathrm{~kg} / \mathrm{m}^{2}$ bei der ersten Untersuchung, bei einer positiven Familienanamnese für Präeklampsie oder bei multiplen Schwangerschaften. Bei hohem oder mäßiggradigem Risiko für Präeklampsie wird die prophylaktische Einnahme von 75 [480] -100-150 mg Aspirin täglich von Woche 12 bis 36-37 empfohlen [481, 482].

Eklampsie und HELLP Syndrom sind die schwersten Komplikationen einer Präeklampsie. Etwa 1-2\% der Schwangerschaften mit Präeklampsie wird durch Eklampsie - zerebrale Krampfanfälle (Grand mal Anfälle) - kompliziert. Das HELLP Syndrom - Hämolyse, Elevierte Leberenzyme, Low Plateletes (Thrombozytopenie) - wird durch eine akute endotheliale Dysfunktion verursacht, hervorgerufen durch plazentare Faktoren [52].

\section{Hypertonie: Diagnostik und Überwachung}

Die Messung des BDs bietet bei Schwangeren besondere Probleme. Auch validierte oszillometrische Messgeräte - dies gilt auch für ABDM- und Selbstmessgeräte - sind für Schwangere, insbesondere solche mit Präeklampsie, oft ungenau [52, 483-485]. Es sollen daher nur Geräte verwendet werden, die explizit für Schwan- 
Tab. 29 Indikationen zu medikamentöser Blutdrucksenkung und Zielwerte (Office-Blutdruck)

\begin{tabular}{|c|c|c|}
\hline Klinischer Zustand & Indikation zu Blutdruck-Senkung (mm Hg) & Zielwert (mm Hg) \\
\hline Keine Organschäden, keine Präeklampsie, unkomplizierte Gravidität & $\geq 150 / \geq 100^{\mathrm{a}}$ & $<150 / 100$ \\
\hline Chronische Hypertonie mit Organschäden & $\geq 140 / \geq 90^{b}$ & $130-140 /<90$ \\
\hline Präeklampsie & $\geq 150 / \geq 100$ & $130-150 /<100$ \\
\hline
\end{tabular}

gere validiert sind (siehe: www.dableducational.org). Ein Ordinations-BD von $\geq 140$ /oder $\geq 90 \mathrm{~mm} \mathrm{Hg}$, an mindestens 2 Terminen festgestellt, gilt als Hypertonie. Die Messung muss im Sitzen unter strikter Beachtung aller Standards erfolgen [472].

Bei etwa einem Drittel der Schwangeren kann eine Weißkittelhypertonie beobachtet werden, was ohne Notwendigkeit zu medikamentöser Blutdrucksenkung und vermehrt zu Kaiserschnitt-Entbindungen führt [486]. Es ist deswegen nachdrücklich eine Diagnosesicherung durch ABDM zu fordern. Normalwerte für ABDM betragen bis zur 32. Woche unter Tags $116 \pm 9 / 70 \pm 7 \mathrm{~mm} \mathrm{Hg}$ und nachts $100 \pm 8 / 55 \pm 6 \mathrm{~mm} \mathrm{Hg}$; von der 33. bis zur 40. Woche steigen die entsprechenden Werte um etwa $4 \mathrm{~mm} \mathrm{Hg}$ an [487]. ABDM Werte weisen eine bessere Voraussage zum Risiko einer vorzeitigen Beendigung der Schwangerschaft, eines niedrigen Geburtsgewichts und eine bessere Korrelation zu hypertensiven Komplikationen und Proteinurie als Praxiswerte auf [486-488].

Die Selbstmessung kann zur Blutdrucküberwachung verwendet werden, vorausgesetzt dass eine gute Übereinstimmung mit ABDM Tages-Werten sowie mit der Office Messung bei simultaner Messung gefunden wurde.

Die Diagnostik umfasst die übliche Routine bei Hypertonie, spezielle Bedeutung kommt folgenden Befunden zu [481]: Harnuntersuchung, Blutbild (Hämatokrit, Thrombozyten), Leberenzyme, Kreatinin, Harnsäure (ist bei Präeklampsie meist erhöht und ein Risikomarker für fötale und mütterliche Komplikationen [489]). Erhöhter BD sollte 2 Mal wöchentlich von der Ärztin/dem Arzt gemessen werden, bei Selbstmessung öfter. Bei Schwangeren mit Hypertonie und Präeklampsie soll die Entwicklung des Fetus regelmäßig sonographisch kontrolliert werden.

\section{Therapie der Hypertonie in der Schwangerschaft}

Werte $\geq 170 \mathrm{~mm}$ Hg systolisch bzw. $\geq 110 \mathrm{~mm}$ Hg diastolisch gelten als Notfall und erfordern eine sofortige Hospitalisierung mit engmaschiger Überwachung von Mutter und Fötus [481]. Die erforderliche BD-Senkung muss graduell erfolgen, um die Blutversorgung des Fötus nicht zu gefährden [476].

Ein systolischer BD $>160 \mathrm{~mm} \mathrm{Hg}$ oder ein diastolischer $\mathrm{BD}>100-110 \mathrm{~mm} \mathrm{Hg}$ wird unabhängig von der Ursache der Hypertonie als schwer und somit behandlungsbedürftig angesehen, um das mütterliche Risiko zu reduzieren [490], insbesonders des ischämischen oder hämorrhagischen Insults, der im Zusammenhang mit der Präeklampsie droht.

Die Prognose von Schwangeren mit chronischer primärer Hypertonie und normaler Nierenfunktion ohne Präeklampsie ist gut. Bei den meisten Frauen mit leichter Hypertonie (systolischer BD 140-159 mm Hg, diastolischer BD 90-99 mm Hg) sinkt der BD spontan im 2. Trimenon, eine medikamentöse BD-Senkung ist selten notwendig. Eine Meta-Analyse [490] (49 randomisierte Studien, 4723 Patientinnen) zeigte, dass medikamentöse BD-Senkung bei leichter bis mittelschwerer Hypertonie (140-169/90-109 mm Hg) das Risiko für die Entwicklung einer schweren Hypertonie halbiert. Allerdings werden die Häufigkeit von intrauterinem Fruchttod, vorzeitiger Geburt oder Unterentwicklung des Kindes nicht signifikant reduziert. Auch die Entwicklung einer Präeklampsie wird dadurch nicht sicher beeinflusst. In der CHIPS-Studie [491] wurden 987 Frauen zwischen der 14. und der 33. Schwangerschaftswoche mit einem diastolischen Office-BD von $90-105 \mathrm{~mm} \mathrm{Hg}$ (unter Therapie 85-105 mm Hg) und einem systolischen BD unter $160 \mathrm{~mm} \mathrm{Hg}$, aber ohne Proteinurie, zu einem diastolischen Blutdruckziel von 100 versus $85 \mathrm{~mm} \mathrm{Hg}$ randomisiert. Der $\mathrm{BD}$ vor Randomisierung lag bei etwa 140/93 mm Hg. Primärer Endpunkt war die Kombination aus Verlust des Fötus und neonatologische Intensivtherapie für mehr als $48 \mathrm{~h}$. Sekundärer Endpunkt waren schwere mütterliche Komplikationen. Der erreichte BD lag bei $139 / 90 \mathrm{~mm} \mathrm{Hg}$ versus 133/85 mm Hg. Sowohl der primäre als auch der sekundäre Endpunkt trat in beiden Gruppen gleich häufig auf, ebenso niedriges Geburtsgewicht. Das Risiko für schwere Hypertonie lag in der Gruppe mit dem höheren Blutdruckziel um 80 \% höher. Eine Nachanalyse der CHIPS-Studie [492] zeigte, dass das Auftreten einer schweren Hypertonie ein Marker für mütterliche oder fötale Komplikationen ist, auch unabhängig vom Vorliegen einer Präeklampsie.

Bei nicht schwerer chronischer Hypertonie und dem zu erwartenden spontanen Abfall des BDs zu Beginn der Schwangerschaft kann eine laufende Medikation versuchsweise abgesetzt werden, muss aber meist gegen Ende der Schwangerschaft wegen ansteigender BD-Werte wieder begonnen werden. $\mathrm{Zu}$ beachten ist, dass aufgrund der in der Schwangerschaft veränderten Metabolik und Elimination die meisten Medikamente kürzer und schwächer wirksam sind [493]. 
Tab. 30 Antihypertensiva der ersten Wahl in der Schwangerschaft

\begin{tabular}{|l|l|l|}
\hline Medikament & Tägliche Dosis & Nebenwirkungen \\
\hline Methyldopa (Aldometil ${ }^{\circledR}$ ) & $250-500 \mathrm{mg} 2-4$ Mal, max. $2000 \mathrm{mg}$ & Depression, Leberstörungen, selten hämolytische Anämie \\
\hline Labetalol (Trandate $\left.{ }^{\circledR}\right)$ & $200-400 \mathrm{mg} 2-4 \mathrm{Mal}$, max. $1200 \mathrm{mg}$ & Ev. fötale Wachstumsretardierung \\
\hline Nifedipin ret. (Adalat ret. $\left.{ }^{\circledR}\right)$ & $10-20 \mathrm{mg}, 2-3$ Mal, max. $180 \mathrm{mg}$ & Kann Wehen hemmen \\
\hline
\end{tabular}

Tab. 31 Notfallmedikation bei schwerer Hypertonie und Präeklampsie [475, 481, 497]

\begin{tabular}{|c|c|}
\hline $\begin{array}{l}\text { Urapidil } \\
\text { (Ebranti|®) }\end{array}$ & $12,5-25 \mathrm{mg}$ iv Bolus, gefolgt von Dauerinfusion mit $5-30 \mathrm{mg} /$ Stunde \\
\hline $\begin{array}{l}\text { Labetalol } \\
\left.\text { (Trandate }{ }^{\circledR}\right)\end{array}$ & $\begin{array}{l}10-20 \mathrm{mg} \text { iv Bolus, Wiederholung alle 10-20 min wenn notwendig in steigender Dosis bis } 80 \mathrm{mg} \text { oder Dauerinfusion mit } 1-2 \mathrm{mg} / \mathrm{min} \text {, bis } \\
\text { maximal } 300 \mathrm{mg}\end{array}$ \\
\hline $\begin{array}{l}\text { Nifedipin } \\
\left.\text { (Adalat }{ }^{\circledR}\right)\end{array}$ & $\begin{array}{l}\text { 5-10 mg Kapseln oral (nicht sublingual wegen Gefahr abrupter Blutdrucksenkung), wenn erforderlich nach 20-30 min weitere 10-20 mg } \\
\text { oral, maximal } 80 \mathrm{mg} / \mathrm{Tag} \text { bzw. 0,63-1,25 mg/h iv (Lichtschutz!) }\end{array}$ \\
\hline Hydralazin ${ }^{a}$ & $\begin{array}{l}\text { 2,5-5 mg iv, Wiederholung mit 5-10 mg alle } 20-30 \mathrm{~min} \text { solang erforderlich } \\
\text { Vor der ersten Applikation wird Volumenzufuhr ( } 500 \mathrm{ml}) \text { empfohlen um eine zu starke Blutdrucksenkung abzufangen }\end{array}$ \\
\hline \multicolumn{2}{|c|}{$\begin{array}{l}\text { Urapidil hat sich in } 2 \text { kleineren Studien als wirksam und risikolos für Mutter und Kind erwiesen [498, 499]. In seltenen Fällen kann auch Na-Nitroprussid unter } \\
\text { Beachtung der üblichen Kautelen verwendet werden [475] } \\
\text { aHydralazin verursacht vermehrt perinatale Probleme [500] und wird nicht mehr als Medikament der ersten Wahl empfohlen, nur noch als Reservemedikament } \\
\text { nach Versagen anderer Antihypertensiva }\end{array}$} \\
\hline
\end{tabular}

\section{Nicht-pharmakologische Behandlung}

Diese ist prinzipiell immer vorrangig zu empfehlen, insbesondere wenn bei leichter Hypertonie keine medikamentöse Therapie erforderlich ist [468]. Streng salzarme Kost gilt wegen der Gefahr einer Hypovolämie als kontraproduktiv, eine moderate Na-Einschränkung kann empfohlen werden [480]. Prolongierte Bettruhe ist ebensowenig indiziert wie Gewichtsreduktion bei adipösen Schwangeren. Empfohlen wird ausgewogene Kost und absoluter Verzicht auf Alkohol und Zigaretten. Regelmäßige leichte bis mittelschwere körperliche Aktivität ist wegen einer Reihe von positiven Effekten anzuraten [494].

\section{Medikamentöse Blutdrucksenkung}

Primäres Ziel der Blutdrucksenkung ist die Vermeidung von mütterlichen Komplikationen, wobei die ausgewählten Medikamente sicher für den Fötus sein sollen.

Unter Berücksichtigung leicht divergierender Empfehlungen diverser Fachgesellschaften [480, 481] schlagen wir die in Tabelle 29 angeführten Indikationen und Zielwerte einer medikamentösen Blutdrucksenkung vor. Eine zu ehrgeizige Blutdrucksenkung ist $\mathrm{zu}$ vermeiden, da bei Werten unter $130 / 80 \mathrm{~mm} \mathrm{Hg}$ Mangeldurchblutung der Plazenta droht [464].

\section{Medikamentenauswahl}

Für alle Formen der Hypertonie in der Gravidität werden international in erster Linie Labetalol, ein nichtselektiver $\beta$-Blocker mit $\alpha$-Rezeptoren blockierender Kapazität (in Österreich derzeit nicht am Markt), $\alpha$-Methyldopa und retardiertes Nifedipin (von NICE nicht vor der $20 \mathrm{SSW}$ ) empfohlen (siehe Tab. 30). $\alpha$-Methyldopa wird nur bedingt als Mittel der ersten Wahl betrachtet [490] und sollte besonders bei Frauen mit
Depression vermieden werden. In einer Meta-Analyse (11 Studien) konnte gezeigt werden, dass die Kombination von Betablocker mit einem Kalziumantagonisten (aber nicht deren isolierte Gabe) besser als $\alpha$-Methyldopa das Auftreten von Proteinurie/Präeklampsie verhindert [490]. Als Mittel der zweiten Wahl gelten Hydralazin, Alphablocker und mit Einschränkungen einige Betablocker (Oxprenolol, Metoprolol). Atenolol sollte wegen der erhöhten Gefahr einer retardierten Entwicklung des Fetus in der ersten Schwangerschaftshälfte vermieden werden [493]. Auch eine Reihe weiterer Kalziumantagonisten (u. a. Isradipin, Verapamil) ist verwendet worden. Clonidin kann als Drittmedikation bei therapierefraktärer Hypertonie eingesetzt werden. Die angeführten Medikamente der 2. und 3. Wahl kommen vor allem als Kombinationspartner bei ungenügender Wirksamkeit der Mittel der ersten Wahl zum Einsatz. Alle genannten Medikamente scheinen keine teratogenen Effekte zu haben und gelten als einigermaßen sicher.

ACE-Hemmer und ARBs sind im 1. Trimenon für den Fötus teratogen und beträchtlich toxisch und ebenso kontraindiziert wie direkte Reninhemmer. Spironolacton passiert die Plazenta, verursacht genitale Missbildungen durch antiandrogene Effekte und ist ebenfalls kontraindiziert. Amilorid wurde vereinzelt bei Graviden mit Hyperaldosteronismus ohne Probleme für Mutter und Kind angewendet [495]. Niedrig dosierte Diuretika werden aus theoretischen Überlegungen nicht empfohlen, wiewohl weder bei Fortsetzung einer entsprechenden Therapie noch in einer randomisierten Studie nachteilige Effekte beobachtet wurden [496].

Die Notfallmedikamente bei schwerer Hypertonie und Präeklampsie zeigt Tab. 31. 


\section{Blutdruck Post Partum}

Nach der Entbindung sollte auf Methyldopa wegen seiner Depression fördernden Nebenwirkung verzichtet werden, dafür kann wieder Enalapril eingesetzt werden [480]. Alle Antihypertensiva werden in die Muttermilch abgegeben, vor allem hoch dosiertes Propranolol und Nifedipin. Die NICE-Guidelines 2019 halten bei spärlicher Datenlage in der postpartalen Stillphase Labetalol, Nifedipin, Amlodipin, Enalapril oder Atenolol für anwendbar [480]. Sobald sich die Schwangerschaftshypertonie, meist binnen 6 Wochen zurückgebildet hat, kann die antihypertensive Therapie beendet werden.

\section{Langzeitfolgen}

Der BD von Frauen mit Schwangerschaftshypertonie sollte jährlich kontrolliert werden, da sie später häufiger eine behandlungspflichtige Hypertonie entwickeln. Außerdem muss sowohl bei Patientinnen mit wie auch ohne Präeklampsie vermehrt mit dem Auftreten von kardiovaskulären Erkrankungen (KHK, tödlicher Myokardinfarkt, Herzinsuffizienz, ischämischer Insult), Niereninsuffizienz und Diabetes gerechnet werden [501]. Neben einer Beratung betreffend Lebensstilmaßnahmen sind Laborkontrollen der kardiovaskulären Risikofaktoren in etwa 3-5 jährlichen Intervallen angezeigt.

Kinder, die im Rahmen einer Präeklampsie geboren wurden, weisen von Jugend an eine Neigung zu erhöhten kardiovaskulären Risikofaktoren auf und sollten entsprechend beraten und überwacht werden [501].

\section{Orale Kontrazeption und Blutdruck}

Kombinierte Östrogen-Progesteron-hältige orale Kontrazeptive können einen signifikanten, aber meist geringen BD-Anstieg verursachen [502], der nach Absetzen der Medikamente reversibel ist. Dies kann besonders beim Vorliegen einer resistenten Hypertonie eine Rolle spielen [503].

\section{Hormonersatztherapie und Blutdruck}

Aus epidemiologischen Untersuchungen ist lange bekannt, dass das Hypertonierisiko bei Frauen nach der Menopause steigt [504]. Es gibt keine überzeugende Evidenz, dass östrogenhältige Hormonersatztherapie den BD bei normotensiven oder hypertensiven menopausalen Frauen relevant erhöht [505]. Hormonersatztherapie ist daher auch bei menopausalen Frauen mit Hypertonie nicht kontraindiziert [52].

\section{Blutdruck bei Kindern und Jugendlichen}

\section{Einleitung und Ziel}

Das Vorliegen einer arteriellen Hypertonie ist bei Kindern und Jugendlichen im Vergleich zu Erwachsenen seltener und wird in entwickelten Ländern mit einer Inzidenz von $2-5 \%$ angegeben. Der Body Mass Index gilt dabei als stärkster bestimmender Faktor für die Erhöhung des BDs bei Kindern und Jugendlichen. Durch die Zunahme der Anzahl von übergewichtigen und adipösen Kindern und Jugendlichen in den letzten Jahrzehnten ist der BD bei dieser Altersgruppe nun auch mehr in den Vordergrund gerückt. Es kann zwar keine direkte Kausalität zwischen kindlicher Hypertonie und späteren kardiovaskulären Ereignissen, wie Myokardinfarkt und cerebralem Insult, hergestellt werden, jedoch können Hypertonie-vermittelte Organschäden (HVOS) in jedem Alter nachgewiesen werden (Tab. 32). Diese können noch diskret und reversibel, jedoch bei schwerer Hypertonie auch lebensbedrohlich sein. Deshalb sind Blutdruckmessungen auch im Österreichischen Mutter-Kindpass im dritten, vierten und fünften Lebensjahr vorgesehen. Weiters wird in den Europäischen Leitlinien bei gesunden Kindern eine Kontrolle des BDs zumindest alle zwei Jahre empfohlen [506].

Die Hypertonie zeigt typischerweise ein TrackingPhänomen, also ein Fortbestehen ins Erwachsenenalter und ist damit auch ein Transitionsthema [507]. Es ist daher Aufgabe aller primär internistisch tätigen Ärztinnen und Ärzten, die mit Kindern und Jugendlichen zu tun haben (Allgemeinmedizin, Kinderund Jugendheilkunde, Innere Medizin), die Problematik der Hypertonie dieser Bevölkerungsgruppe zu kennen.

Risikofaktoren und Ursachen für eine Hypertonie sind vielfältig und in der ausführlichen Anamneseliste aufgelistet (Tab. 33).

Die Basisabklärung und eventuelle Behandlung kann, bzw. sollte von allen oben angeführten Ärztinnen und Ärzten erfolgen, die Abklärung und Betreuung von sekundärer Hypertonie oder Hypertonie Grad 2 sollte in der Hand von Spezialist/innen (der Pädiatrischen Nephrologie oder Kardiologie) liegen.

\section{Definitionen}

Im Gegensatz zu Erwachsenen wird bei Kindern und Jugendlichen bis zum Alter von 16 Jahren die Hypertonie als rein statistische Überschreitung von geschlechts-, alters- und längenabhängigen Referenzwerten (Perzentilen) beschrieben und stellt somit eine dynamische Größe dar. Die dafür am besten evaluierten Referenzwerte sind die in Deutschland an normalgewichtigen und gesunden Kindern erhobenen Werte (Tab. 34; [508]). Ab dem Alter von 16 Jahren werden die Grenzwerte für Erwachsene herangezogen (Tab. 35; [506]).

\section{Leitsymptome, klinische Präsentation}

Meist ist die arterielle Hypertonie symptomlos und ein Zufallsbefund. Beschriebene Symptome sind oft unspezifisch (z.B. Kopfschmerzen, Nasenbluten, 
Tab. 32 Hypertonie-vermittelte Organschäden im Kindesund Jugendalter (in jedem Alter möglich)

\begin{tabular}{|c|c|}
\hline \multirow[t]{2}{*}{ Endorganschäden } & Untersuchungen \\
\hline & Obligat bei Erstabklärung \\
\hline $\begin{array}{l}\text { Linksventrikel- } \\
\text { hypertrophie }\end{array}$ & Echokardiographie: Linksventrikulärer Massenindex \\
\hline \multirow[t]{2}{*}{ Albuminurie } & $\begin{array}{l}\text { Harnchemie (primär mit Streifentest möglich), präzi- } \\
\text { ser durch Albumin/Kreatinin Ratio (normal <30 mg/g) } \\
\text { nachweisbar }\end{array}$ \\
\hline & Weiterführende Untersuchungen \\
\hline $\begin{array}{l}\text { Fundus hyperto- } \\
\text { nicus }\end{array}$ & $\begin{array}{l}\text { Fundoskopie, (reversible) Veränderungen auch bei } \\
\text { Kleinkindern nachweisbar }\end{array}$ \\
\hline $\begin{array}{l}\text { Erhöhte Intima- } \\
\text { Media-Dicke }\end{array}$ & Sonographie \\
\hline $\begin{array}{l}\text { Pulswellen- } \\
\text { geschwindigkeit }\end{array}$ & Messung im Rahmen der $24 \mathrm{~h}$ ABDM möglich \\
\hline $\begin{array}{l}\text { Hypertensive } \\
\text { Enzephalopathie }\end{array}$ & Magnetresonanztomographie \\
\hline
\end{tabular}

Schwindel, Tinnitus, Konzentrationsstörungen) und können auch auf völlig andere Erkrankungen hinweisen, sollten aber immer zu einer Messung des BDs veranlassen.

\section{Messmethoden und Referenzwerte}

Die erwähnten Referenzwerte wurden oszillometrisch erhoben, was auch der gängigen Praxis in Klinik und Ordination entspricht (Tab. 34). Diese Referenzwerte weichen etwas von den derzeit bestehenden pädiatrischen Empfehlungen der European Society of Hypertension ab [506]. Diese beziehen sich nämlich auf auskultatorisch erhobene Werte US-amerikanischer (auch übergewichtiger) Kinder [509].

Die Messungen sollten immer in ausreichender Ruhe (mind. 5 min Sitzen) und mit adäquaten Manschetten (Säuglingsgröße bis Erwachsenengrößen) erfolgen. In Ordinationen und Ambulanzen gemessene Werte sind häufig zunächst im hypertensiven Bereich. Bei wiederholten Messungen zeigen sich jedoch normale Werte. Deshalb beinhaltet eine adäquate Messung drei Werte (3 min Abstand), wobei der Durchschnitt der letzten beiden Messungen herangezogen wird. Bei hypertensiven Werten sollte eine auskultatorische Bestätigung erfolgen.
Tab. 33 Anamnese bei Hypertonie im Kindes- und Jugendalter

\begin{tabular}{|c|c|}
\hline Anamnese & Frage nach: \\
\hline \multirow{5}{*}{$\begin{array}{l}\text { Familien- } \\
\text { anamnese }\end{array}$} & Hypertonie \\
\hline & $\begin{array}{l}\text { Herz-Kreislauf-Erkrankungen (Myocardinfarkt, Insult) } \\
\text { Frage nach Zeitpunkt des Ereignisses }\end{array}$ \\
\hline & Nierenerkrankungen \\
\hline & $\begin{array}{l}\text { Diabetes mellitus } \\
\text { Fettstoffwechselerkrankungen } \\
\text { Adipositas } \\
\text { Familiäre Lebensgewohnheiten z. B. Elterliches Rau- } \\
\text { chen }\end{array}$ \\
\hline & $\begin{array}{l}\text { IVF-Schwangerschaft, mütterliche Hypertonie und } \\
\text { Präekklampsie } \\
\text { Steroidbehandlung (Lungenreifung) } \\
\text { Mütterlicher Substanzabusus }\end{array}$ \\
\hline $\begin{array}{l}\text { Peri- und Neona- } \\
\text { talperiode }\end{array}$ & $\begin{array}{l}\text { Frühgeburtlichkeit/niedriges Geburtsgewicht } \\
\text { Nabelarterienkatheter } \\
\text { Beatmung (Bronchopulmonale Dysplasie) }\end{array}$ \\
\hline $\begin{array}{l}\text { Vorerkrankungen/ } \\
\text { Operationen }\end{array}$ & $\begin{array}{l}\text { Renale Vorerkrankungen } \\
\text { Harnwegsinfekte und Miktionsstörungen } \\
\text { Op. an Nieren und Harnwegen } \\
\text { Akutes Nierenversagen } \\
\text { Nephritis (Ödeme? Hämaturie?) } \\
\text { Chronische Nierenerkrankungen und } \\
\text { Nierentransplantation } \\
\text { Nierenvenenthrombose } \\
\text { Kardiale und vaskuläre Vorerkrankungen } \\
\text { Aortenisthmusstenose } \\
\text { Persistierender Ductus Arteriosus } \\
\text { Mid aortic Syndrom } \\
\text { Sichelzellanämie } \\
\text { Knochemarkstransplantation }\end{array}$ \\
\hline $\begin{array}{l}\text { Aktuelle } \\
\text { Beschwerden }\end{array}$ & $\begin{array}{l}\text { Cephalea, Tages-Müdigkeit, Flush-Symptome, Nasen- } \\
\text { bluten, Herzrhythmusstörungen, Palpitation } \\
\text { Gewichtsabnahme, Starkes Schwitzen }\end{array}$ \\
\hline $\begin{array}{l}\text { Lebensgewohn- } \\
\text { heiten }\end{array}$ & $\begin{array}{l}\text { Ernährung } \\
\text { Trinkverhalten } \\
\text { Schlaf, Schnarchen } \\
\text { Rauchen } \\
\text { Bewegung, Sport } \\
\text { Bildschirmzeit }\end{array}$ \\
\hline Medikamente & $\begin{array}{l}\text { Blutdruckerhöhende Medikamente } \\
\text { Psychostimulation, } \\
\text { Tricyklische Antidepressiva } \\
\text { Steroide } \\
\text { Phytopharmaka } \\
\text { Relevante frühere Medikation (z. B. Chemotherapie) }\end{array}$ \\
\hline
\end{tabular}

Tab. 34 Referenzwerte bei gesunden Knaben und Mädchen

\begin{tabular}{|c|c|c|c|c|}
\hline & \multicolumn{2}{|l|}{ Knaben } & \multicolumn{2}{|l|}{ Mädchen } \\
\hline Alter & 50. Perzentile $(\mathrm{mm} \mathrm{Hg})$ & 95. Perzentile (mm Hg) & 50. Perzentile $(\mathrm{mm} \mathrm{Hg})$ & 95. Perzentile $(\mathrm{mm} \mathrm{Hg})$ \\
\hline 3 Jahre & $96 / 58$ & $110 / 70$ & $96 / 58$ & $110 / 70$ \\
\hline 6 Jahre & $100 / 60$ & $112 / 70$ & $98 / 60$ & $112 / 72$ \\
\hline 9 Jahre & $102 / 62$ & $118 / 74$ & $102 / 62$ & $118 / 74$ \\
\hline 12 Jahre & $108 / 66$ & $125 / 76$ & $108 / 66$ & $124 / 76$ \\
\hline 15 Jahre & $113 / 68$ & $128 / 80$ & $113 / 68$ & $128 / 80$ \\
\hline$\geq 16$ Jahre & \multicolumn{2}{|l|}{ Normal $<130 / 85$} & \multicolumn{2}{|c|}{ Hochnormal 130-139/85-89 } \\
\hline
\end{tabular}


Tab. 35 Klassifikation und Grenzwerte des Blutdrucks bei Kindern und Jugendlichen

\begin{tabular}{l|ll|} 
& Bis $<16$ Jahre & $\geq 16$ Jahre $(\mathrm{mm} \mathrm{Hg})$ \\
\hline Normal & $<90$. Perzentile & $<130 / 80-84$ \\
\hline Hochnormal & $90 .-94$. Perzentile & $130-139 / 85-89$ \\
\hline Hypertonie Grad 1 & 95.-99. Perzentile $+5 \mathrm{~mm} \mathrm{Hg}$ & $140-159 / 90-99$ \\
\hline Hypertonie Grad 2 & $>99$. Perzentile $+5 \mathrm{~mm} \mathrm{Hg}$ & $160-179 / 100-109$
\end{tabular}

Auch bei Kindern und Jugendlichen haben häusliche Messungen einen wichtigen Stellenwert bei der Einstellung und Überwachung einer manifesten Hypertonie [510]. Eine Verordnung und Refundierung eines adäquaten Gerätes ist daher bei entsprechender Argumentation über die Kassen möglich.

\section{Ambulantes 24-h-Blutdruckmonitoring (24h ABDM) als diagnostischer Standard}

Wenn trotz Mehrfachmessungen in der Ordination oder Ambulanz hypertensive Werte erhoben werden, sollte ein $24 \mathrm{~h}$ ABDM erfolgen, da sich dieses dann in bis zu $50 \%$ der Fälle als normal erweist (z. B. Weißkittelhypertonie) [511]. Dies kann unnötige wei-
Tab. 36 Einsatz des ambulanten 24-h-Blutdruckmonitorings (24h ABDM) bei Kindern und Jugendlichen

Bestätigung einer Hypertonie, vor weiterer Abklärung und Behandlung

Diabetes mellitus

Bei Zielorganschäden trotz normalem Blutdruck in Ambulanz oder Ordination (maskierte Hypertonie, nächtliche Hypertonie)

Chronische Nierenerkrankung

Nach Organtransplantation

Schwere Adipositas mit oder ohne Schlafapnoe

Bei Unklarheit über Effektivität der antihypertensiven Behandlung und Kontrolle der circadianen Blutdruckeinstellung

tere Abklärungen und nicht indizierte Behandlungen vermeiden. Dies gilt nicht für eine schwere, sofort behandlungsbedürftige bzw. krisenhafte Hypertonie (20 mm Hg über der 95. Perzentile) [512].

Ein $24 \mathrm{~h}$ ABDM sollte auch bei Kindern mit entsprechenden Risikokonstellationen trotz normaler Werte bei Messung in Ordination oder Ambulanz durchgeführt werden, weil hier auch eine maskierte, vor allem nächtliche Hypertonie vorliegen könnte (Tab. 36). Für die Beurteilung der $24 \mathrm{~h}$ ABDM sollten die entspreche-

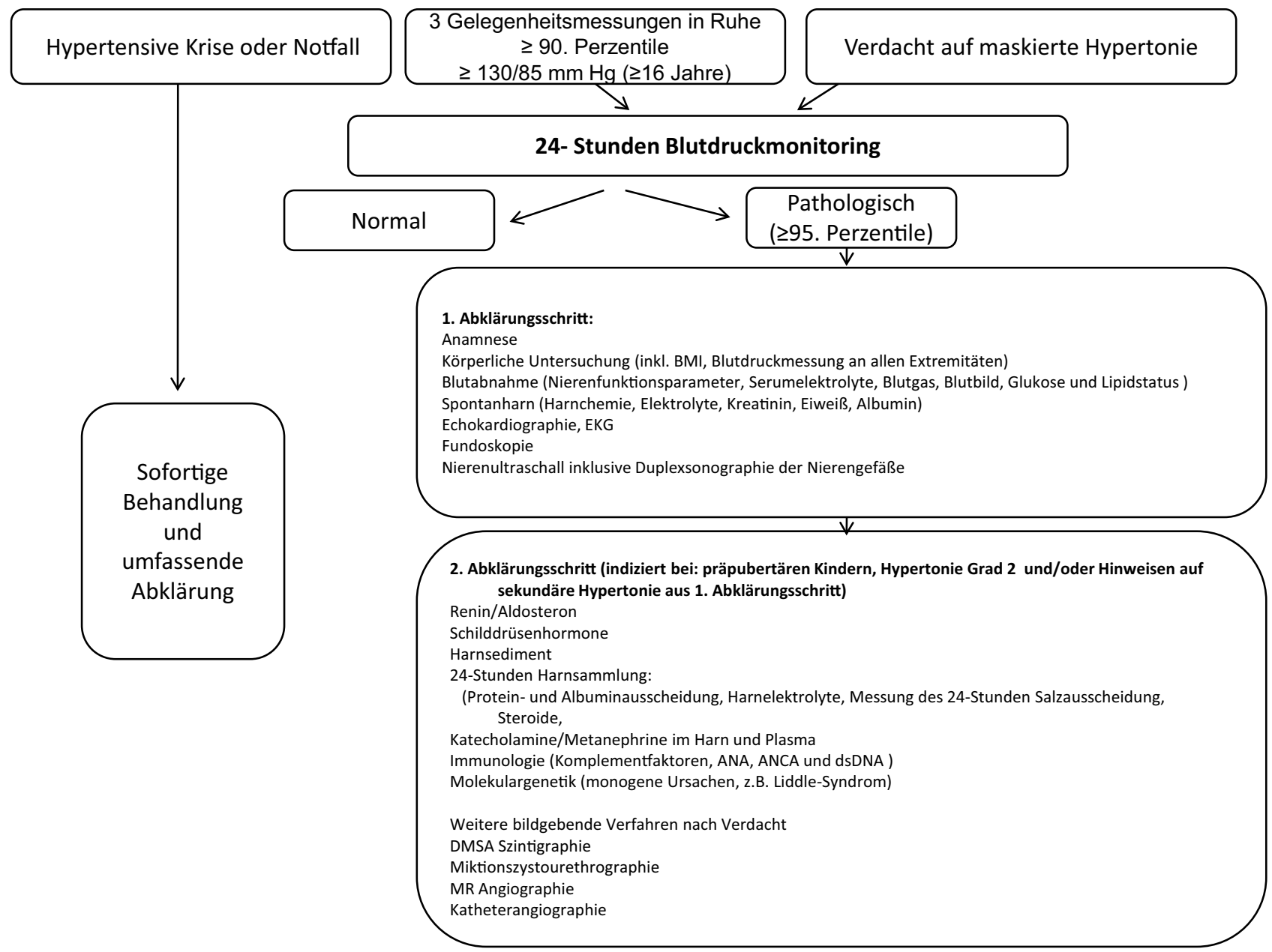

Abb. 6 Hypertonie-Abklärung bei Kindern und Jugendlichen 
Tab. 37 Lebensstil-Ziele bei Kindern und Jugendlichen

BMI <85. Perzentile
Rauchfreie Umgebung
Ernährung: z. B. „DASH-Diät“
Geringer Gehalt
An Fleisch,
Gesamtfett und Cholesterin,
Gesättigten Fettsäuren und
Gesüßten Getränken,
Hoher Gehalt
An Protein, Fisch, Geflügelfleisch,
Vollkornprodukten, Ballaststoffen, Kalium, Calcium und Magnesium.
Salzrestriktion
Bei Kindern und Jugendlichen mit Hypertonie ist mit 1 g weniger Na Zu-
fuhr/Tag eine Reduktion des Blutdrucks von systolisch 6,3 mm Hg, diasto-
lisch 3,5 mm Hg möglich [515]
Physikalische Aktivität
60 min mïtlere bis intensive körperliche Aktivität/Tag
Einschränkung von Wettkampfsport nur bei unkontrollierter Hypertonie
Stadium 2
Tägliche Bildschirmzeit unter zwei Stunden

nenden Referenzwerte (für Kinder ab $120 \mathrm{~cm}$ Länge) von Wühl et al. herangezogen werden [513].

\section{Stufenweise Abklärung}

Eine zentrale Rolle nimmt die Anamnese ein, da hier schon oft mögliche Ursachen der Hypertonie erhoben werden können (Tab. 33). Der erste Abklärungsschritt zielt auf die Aufdeckung von (häufigeren) Ursachen und dient der Feststellung von Komorbiditäten (z. B. Diabetes mellitus, Adipositas). Weiters zielt er auf den Nachweis von eventuell schon vorhandenen HVOS. Hier stellt die Echokardiographie eine wichtige Rolle, auch in Bezug auf Ursachensuche dar (Tab. 32, Abb. 6).

Eine Hypertonie im präpubertären Alter besteht in der Regel als Symptom einer zugrundeliegenden Erkrankung. Am häufigsten sind renoparenchymatöse, gefolgt von renovaskulären und kardialen Erkrankungen (z.B. Aortenisthmusstenose), während bei Jugendlichen der Anteil an primärer Hypertonie höher einzuschätzen ist.

Bei präpubertären Kindern ist also eine erweiterte Abklärung nach Bestätigung durch das 24 h ABDM immer indiziert. Bei Jugendlichen mit Hypertonie Grad 1 kann zunächst auf eine erweiterte Abklärung verzichtet werden. Eine schwere Hypertonie (ab Grad 2) oder Hinweisen auf eine sekundäre Hypertonie aus der Anamnese oder dem ersten Abklärungsschritt bedarf auch bei dieser Altergruppe einer eingehenden Abklärung.

Die Auswahl der Untersuchungen sollte aber den klinischen Verdachtsmomenten folgen (z.B. DMSASzintigraphie bei Verdacht einer Parenchymschädigung durch rezidivierende Pyelonephritiden) (Abb. 6).

\section{Behandlung}

Prinzipielles Ziel der Behandlung ist die Reduktion des Risikos für folgende kardiovaskuläre Ereignisse und

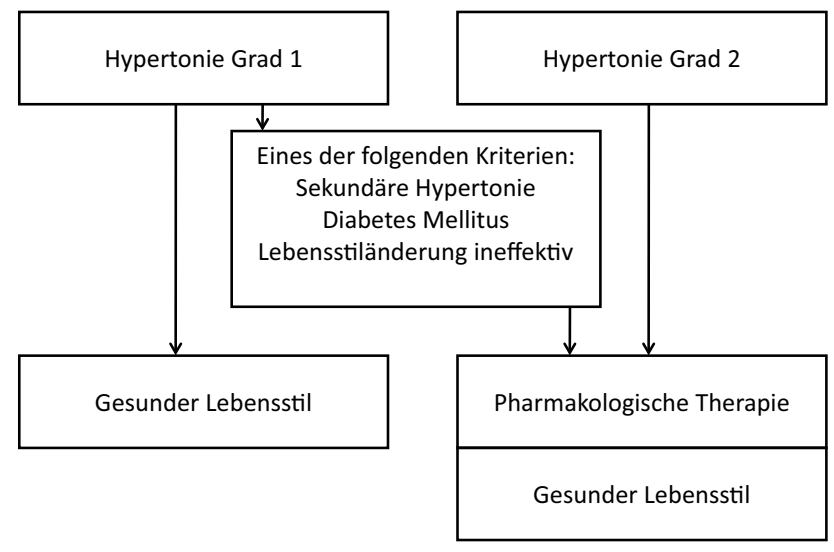

Abb. 7 Behandlungsweg Hypertonie im Kindes- und Jugendalter

die günstige Beeinflussung einer schon bestehenden, vor allem einer renoparenchymatösen Erkrankung. Wie weit die Senkung des BDs erfolgen soll, ist wegen der schwachen Datenlage unsicher. Internationale Empfehlungen und Leitlinien richten sich deshalb teilweise nach Empfehlungen aus Erwachsenenleitlinien und nur zu einem kleinen Teil nach Daten aus pädiatrischen Studien.

Grundsätzlich ist die Senkung des Blutdruckes auf Werte unter der 90. Perzentile oder unter 130/85 mm Hg (ab 16 Jahre) anzustreben. Für Kinder und Jugendliche mit Grunderkrankungen bestehen aber weitere Empfehlungen, die teilweise deutlich unter der 90. Perzentile liegen. So ist das Ziel bei chronischen Nierenerkrankungen mit Proteinurie eine Absenkung an die 50. Perzentile, bei fehlender Proteinurie an die 75. Perzentile [506, 514].

Die Strategien zur Erreichung der Behandlungsziele bestehen aus der (wenn möglichen) Behandlung der Ursache (z.B. Operation eines endokrinen Tumors), der Einhaltung eines gesunden Lebensstils (Tab. 37) und der pharmakologischen Therapie.

\section{Lebensstil}

Eine nichtpharmakologische Therapie (LebensstilModifikation) ist der erste Schritt und Therapie der Wahl bei der Behandlung einer milden primären Hypertonie (Tab. 37).

Die Beeinflussung des Lebensstils, so schwierig es gerade bei Jugendlichen sein mag, stellt eines der wichtigsten und folgenreichsten Ziele dar. Dies gilt vor allem in Hinblick auf die vielen zu erwartenden Lebensjahre und die Risikominderung nicht nur für kardiovaskuläre Erkrankungen. Ein Erreichen eines gesunden Lebensstils wird für die überwiegende Zahl der Betroffenen allerdings nur erreichbar sein, wenn auch Mitspieler, wie Politik, Gesellschaft und Familie (z.B. rauchfreie Umgebung) ihre wichtige Aufgabe hier wahrnehmen. 
Tab. 38 Orale Antihypertensiva bei Kindern (in Österreich zugelassene und/oder gebräuchliche Medikamente)

\begin{tabular}{|c|c|c|}
\hline & Dosierung/Tag (Start- bis Maximaldosis) & Kommentare und Angaben aus Fachinformation (FI) \\
\hline \multicolumn{3}{|c|}{ ACE-Hemmer und ARBS } \\
\hline Captopril & $0,3-6 \mathrm{mg} / \mathrm{kg}$ & Derzeit in Österreich nicht als Monosubstanz erhältlich \\
\hline Enalapril & $0,1-0,5 \mathrm{mg} / \mathrm{kg}$ & Fl: Es können keine spezifischen Dosierungsempfehlungen gegeben werden \\
\hline Ramipril & $1,5-6 \mathrm{mg} / \mathrm{m}^{2}$ & Keine Zulassung für Kinder \\
\hline Lisinopril & $0,08-0,6 \mathrm{mg} / \mathrm{kg}$ & Fl: 6-18 Jahre, 2,5 mg bei $20-50 \mathrm{kgKG}, 5 \mathrm{mg}>50 \mathrm{kgKG}$ \\
\hline Losartan & $0,7-1,4 \mathrm{mg} / \mathrm{kg}$ & Fl: 6-18 Jahre, 25-50 mg (10-50 kgKG) \\
\hline Valsartan & $0,4 \mathrm{mg} / \mathrm{kg}$ & Fl: 6-18 Jahre, 40-160 mg (>35kg) \\
\hline Candesartan & $0,16-0,5 \mathrm{mg} / \mathrm{kg}$ & Fl: 6-18 Jahre, 4-16 mg (>50 kg) \\
\hline \multicolumn{3}{|l|}{ Beta-Blocker } \\
\hline Propanolol & $1-4 \mathrm{mg} / \mathrm{kg}$ & Fl: Zulassung nur für tachykarde Rhythmusstörungen \\
\hline Metoprolol & $0,5-2 \mathrm{mg} / \mathrm{kg}$ & Fl: Unzureichende Daten - nicht empfohlen \\
\hline Bisoprolol & $0,05-0,4 \mathrm{mg} / \mathrm{kg}$ & Fl: Unzureichende Daten - nicht empfohlen \\
\hline Atenolol & $0,5-2 \mathrm{mg} / \mathrm{kg}$ & Fl: Unzureichende Daten - nicht empfohlen \\
\hline Carvedilol & $0,2-1 \mathrm{mg} / \mathrm{kg}$ & Fl: Unzureichende Daten - nicht empfohlen \\
\hline \multicolumn{3}{|l|}{ Kalziumantagonisten } \\
\hline Amlodipin & $0,1-0,5 \mathrm{mg} / \mathrm{kg}$ & Fl: Kinder ab 6 Jahre (Dosis von 2,5-5 mg untersucht) \\
\hline \multicolumn{3}{|l|}{ Diuretika } \\
\hline Furosemid & $0,5-6 \mathrm{mg} / \mathrm{kg}$ & Fl: keine Altersgrenze \\
\hline Hydrochlorothiazid & $0,5-3 \mathrm{mg} / \mathrm{kg}$ & Fi: Unzureichende Daten - nicht empfohlen \\
\hline \multicolumn{3}{|l|}{ Sonstige } \\
\hline Clonidin & $0,2 \mathrm{mg} / \mathrm{kg}$ & Fl: Unzureichende Daten - nicht empfohlen \\
\hline Minoxidil & $0,1-1 \mathrm{mg} / \mathrm{kg}$ & Fl: Kinder ab 12 Jahren \\
\hline Labetolol & $1-3 \mathrm{mg} / \mathrm{kg}$ & Fl: Unzureichende Daten - nicht empfohlen \\
\hline
\end{tabular}

\section{Pharmakologische Therapie}

Bei sekundärer Hypertonie, symptomatischer Hypertonie, nachweisbaren HVOS, sowie höhergradiger Hypertonie sollte primär eine medikamentöse Therapie erfolgen. Bei fehlendem Effekt einer Lebensstiländerung über längere Zeit (zumindest 6 Monate) wird von den meisten Autoren ebenso eine pharmakologische Therapie als notwendig angesehen (Abb. 7). Letztlich liegt hier aber die Entscheidung über die Einleitung einer medikamentösen Therapie im Ermessen des behandelnden Arztes/der behandelnden Ärztin. In jedem Fall sollte die nichtpharmakologische Therapie fortgesetzt werden.

Durch entsprechende Initiativen der EMA ist die Zahl der Zulassungen für antihypertensive Medikamente (AHT) deutlich gestiegen. Daten zur Wirksamkeit von AHT sind in den letzten Jahren vor allem für die Altersgruppe der 6- bis 18-Jährigen generiert worden. Allerdings sind qualitativ hochwertige Studien zur Langzeitanwendung von AHT rar, was dazu führte, dass auch Studiendaten von Erwachsenen zur Anwendung von AHT bei Kindern und Jugendlichen herangezogen wurden. Weiterhin gibt es vor allem keine Evidenz für eine pharmakologische Therapie bei Kindern und Jugendlichen mit primärer Hypertonie über den tatsächlichen Nutzen hinsichtlich späterer kardiovaskulärer Ereignisse. Kritisch muss auch die Tatsache gesehen werden, dass es für kleine und kleinste Patient/innen kaum Langzeitdaten über Wirkung von
AHT, aber auch deren Auswirkungen auf Wachstum und Entwicklung gibt.

Bis es bessere Daten zur Anwendung von AHT bei Kindern gibt, sollten die Substanz-Klassen verwendet werden, für die es auch gute Daten hinsichtlich Wirkung und Nebenwirkungsprofil bei Erwachsenen gibt. Das betrifft die Gruppe der ACE-Hemmer, Angiotensin-Rezeptorblocker, Kalzium-Kanal-Blocker, Betablocker und Diuretika. Hier gibt es zugelassene Substanzen, wobei diese Zulassungen Vorschulkinder meist ausschließen. Grundlage der Substanz-Wahl sollte einerseits die möglichst einfache Anwendung (z.B. Einmalgabe) sein, andererseits auf den pathophysiologischen Hintergrund (z.B. ACE-Hemmer bei Diabetes mellitus) eingehen. Die Verschreibung sollte von der niedrigst möglichen Dosis ausgehend stufenweise angehoben werden und bei unzureichendem Effekt mit einer zweiten Substanz kombiniert werden. Die Kombinationsmöglichkeiten entsprechen den bei Erwachsenen erprobten Kombinationen (ACEHemmer oder Angiotensin-Rezeptor-Blocker mit Hydrochlorothiazid) [100]. Laut Fachinformation ist ein Großteil der Medikamente nicht für Kinder zugelassen, ein Teil (vor allem Angiotensin-Rezeptor-Blocker) sind für Kinder ab 6 Jahre zugelassen und mit groben Dosierungsempfehlungen versehen. Bei den anderen Substanzen gibt es Dosierungsempfehlungen, die aus der pädiatrischen Literatur entnommen wurden (Tab. 38). 


\section{Blutdruck im Alter}

Die bei über 65-Jährigen vorherrschende isolierte systolische Hypertonie (ISH) [246] ist hauptsächlich Folge einer erhöhten Gefäßsteifigkeit. Im Vergleich zu Jüngeren weist der Blutdruck von Älteren stärkere Schwankungen auf, was Ordinationsmessungen weniger verlässlich macht. Wenn immer möglich, sollten daher Selbstmessungen (korrekte Methodik!) oder unbeobachtete automatische Office-Blutdruckmessungen (Werte entsprechen etwa der Selbstmessung) zur Beurteilung herangezogen werden. Prinzipiell profitieren ältere, 65- bis 80-jährige Patient/innen von einer BD-Senkung ähnlich wie jüngere [241]. Die relative Risikoreduktion hinsichtlich kardiovaskulärer Ereignisse war bei Patient/innen jünger und älter als 65 Jahre in einer großen Meta-Analyse dieselbe, aufgrund des höheren Ausgangsrisikos ist die absolute Risikoreduktion sogar bei älteren Patient/innen höher (und damit die Number needed to treat niedriger) [239]. In der SPRINT Studie wurden 2636 Patient/ innen älter als 75 Jahre eingeschlossen [516]. Patient/ innen, die zum intensiveren Blutdruckziel (systolisch $<120 \mathrm{~mm} \mathrm{Hg}$; erreicht wurden $123 \mathrm{~mm} \mathrm{Hg}$ ) randomisiert wurden, profitierten durch eine Reduktion des kombinierten Endpunktes um $34 \%$ und eine Reduktion der Gesamtsterblichkeit um 33\%, im Vergleich zu den Patient/innen in der Standard-Therapiegruppe (systolisches Ziel $<140 \mathrm{~mm} \mathrm{Hg}$, erreicht wurden $135 \mathrm{~mm} \mathrm{Hg}$. Die BD-Werte wurden mit der automatischen unbeobachteten Office-Messung ermittelt, die grob geschätzt $10 \mathrm{~mm} \mathrm{Hg}$ niedrigere Werte als die konventionelle Office-Messung ergibt. Vermittelt wurde diese Risikoreduktion in SPRINT in erster Linie durch eine Prävention der Herzinsuffizienz, möglicherweise zu erklären durch die häufig in dieser $\mathrm{Al}$ tersgruppe eingesetzten Thiaziddiuretika. Gerade bei Thiaziddiuretika muss man aber das Risiko für eine Hyponatriämie bedenken, die eine Muskelschwäche („Adynamie“) als Leitsymptom aufweist und somit wieder das Sturzrisiko signifikant erhöht.

Eine Meta-Analyse (4 Studien, knapp $11.000 \mathrm{~Pa}-$ tient/innen) zum Vergleich einer intensiveren ( $<140 \mathrm{~mm} \mathrm{Hg}$, Ausnahme SPRINT s. oben) versus einer Standardtherapie (140-149 bzw. 140-159 mm Hg, Ausnahme SPRINT s. oben) [517] bei Personen $>65$ Jahre zeigte, dass die intensivere Therapie zu einer $29 \%$ igen Reduktion kardiovaskulärer Ereignisse, einer $33 \%$ igen Reduktion der kardiovaskulären Sterblichkeit und einer $37 \%$ igen Reduktion an Herzinsuffizienz führt. Die Rate an Nebenwirkungen war in beiden Gruppen gleich.

Die Therapie kann den gleichen Algorithmen wie bei den Jüngern folgen. $\mathrm{Zu}$ berücksichtigen sind die bei alten Menschen häufigen Begleitprobleme wie fortgeschrittene atherosklerotische Gefäßerkrankungen, eingeschränkte Nierenfunktion, erhöhte Neigung $\mathrm{zu}$ orthostatischem Blutdruckabfall, Gebrechlichkeit („frailty“) und vielfältige Begleitmedikation mit Poten- zial zu unerwünschten Arzneimittelinteraktionen. Das erfordert besonders zu Therapiebeginn engmaschige Kontrollen des Befindens (Orthostasesymptome?), die Messung des BDs auch im Stehen (wobei der systolische $\mathrm{BD}$ nicht unter $120 \mathrm{~mm} \mathrm{Hg}$ abfallen soll), und Kontrollen der Nierenfunktion. Ein akuter Abfall der Nierenfunktion ist nicht selten, ob man ärztlicherseits darauf reagiert oder nicht, hängt in erster Linie von der glomerulären Filtrationsrate (GFR) vor Beginn der Therapie, der Begleitmedikation (Polypharmazie!) und dem Ausmaß des GFR Abfalls ab. Bei einem Abfall der GFR von $30 \%$ oder mehr sollte die Dosis von ACEHemmern/ARBs mindestens um die Hälfte reduziert bzw. die Therapie ganz beendet werden. Bei Veränderungen um $20 \%$ oder weniger kann die begonnene Therapie fortgesetzt werden. Das 24-h-Blutdruckmonitoring ist hervorragend zur Erkennung hypotensiver Episoden im Alltag geeignet.

Zielwerte sind auf Basis der oben angeführten Studien und Meta-Analysen systolisch 130-140 mm Hg und diastolisch $\quad 60-80 \mathrm{~mm} \quad \mathrm{Hg}$ (Selbstmessung $125-135 / 60-80 \mathrm{~mm} \mathrm{Hg}$ ), sofern gut toleriert. Bei diesen Ziel-Blutdruckwerten fand sich in einer MetaAnalyse bei Patient/innen älter als 75 Jahre [239] (6 Studien, mehr als 11.000 Patient/innen) noch eine Reduktion kardiovaskulärer Ereignisse.

Die Wahl der Medikamente wird in erster Linie durch Komorbiditäten bestimmt. Alphablocker und Schleifendiuretika sollten, wenn nicht aus anderen Gründen indiziert, vermieden werden.

Bei hochbetagten, über 80-Jährigen Patient/innen in einigermaßen gutem Allgemeinzustand kann eine behutsame Senkung von mehrfach sorgfältig gemessenen systolischen Werten von >150 (Selbstmessung $140 \mathrm{~mm} \mathrm{Hg}$ ) auf etwa 130-140 (Selbstmessung 125-135 mm Hg) Schlaganfälle, kardiovaskuläre Ereignisse und Herzinsuffizienz signifikant reduzieren [239]. Eine Therapie sollte bei Hochbetagten mit Monotherapie in geringer Dosis begonnen und bei Verträglichkeit und Bedarf schrittweise gesteigert werden. Auch gebrechliche Patient/innen (,frail“) wurden in den großen Therapiestudien mit alten Patient/innen (HYVET - über 80-jährige Patient/innen, SPRINT über 75-jährige Patient/innen) eingeschlossen, allerdings solche, die noch unabhängig zuhause wohnten und zu den Studien-Kontrollen in die Klinik anreisen konnten. Sowohl in HYVET [518] als auch in SPRINT [516] fand sich, dass gebrechlichere Patient/innen ebenso von der BD-Senkung profitierten wie fittere Patient/innen. Auch die Rate an Nebenwirkungen war in SPRINT in beiden Behandlungsarmen in jeder Frailty-Gruppe gleich. Allerdings waren in SPRINT Diabetiker/innen, Patient/innen nach Schlaganfall und solche mit manifester Herzinsuffizienz ausgeschlossen, somit Patient/innengruppen, bei denen mit vermehrten Nebenwirkungen zu rechnen ist. Weiters war eine Orthostase, definiert als systolischer $\mathrm{BD}<110 \mathrm{~mm}$ Hg nach einer Minute Stehen, ein Ausschlussgrund für die Studie. 
Für Menschen über dem 85. Lebensjahr sowie für pflegebedürftige oder bettlägerige alte Patient/innen können wegen Fehlens von kontrollierten Therapiestudien keine verbindlichen Empfehlungen abgegeben werden. Eine rezente Beobachtungsstudie an einer großen Kohorte [519] ergab, dass eine gute Antihypertensiva-Adhärenz im Vergleich zu einer sehr geringen bei über 85-Jährigen die Gesamtsterblichkeit und kardiovaskuläre Morbidität prozentuell ähnlich senkt wie in der Vergleichskohorte von 70- bis 84-Jährigen, ohne allerdings auf erreichten BD-Werte Bezug $\mathrm{zu}$ nehmen. Eine Blutdrucksenkung muss bei Hochbetagten individuell erfolgen und wird sich zumeist an Begleiterkrankungen orientieren.

Zusammenfassend gilt, dass gerade bei älteren Patient/innen Ärztinnen und Ärzte nicht strikt nach Zielwert therapieren sollten, sondern individuell nach Risiko und Nutzen für die einzelnen Patient/innen. Der Nutzen einer antihypertensiven Therapie ist auch in dieser Altersgruppe dokumentiert, aber die Behandlung erfordert deutlich mehr Fingerspitzengefühl und orientiert sich vor allem am körperlichen Zustand der Patient/innen. Gerade bei älteren gebrechlichen ( $=$ frailen) Patient/innen wird $\mathrm{zu}$ einer besonders vorsichtigen Vorgehensweise mit regelmäßigen Kontrollen geraten.

\section{Hypertensiver Notfall - Hypertensive Krise}

Ein hypertensiver Notfall („hypertensive emergency“) ist eine Situation mit unkontrollierten, in der Regel ausgeprägt hypertensiven Blutdruckwerten, die mit akutem Hypertonie-vermitteltem Organschaden (HVOS) oder Organversagen einhergehen [52, 520, 521]. Davon zu trennen ist eine deutliche BD-Erhöhung ohne akuten Organschaden bzw. ohne ausgeprägte Symptome (Englisch „hypertensive urgencies“, zu Deutsch hypertensive Krise). Obwohl der hypertensive Notfall unbehandelt eine hohe Letalität hat, sind auch Patient/innen mit hypertensiver Krise gefährdet für ein kardiovaskuläres Ereignis [522, 523]. Die meisten Patient/innen mit hypertensiven Notfällen haben eine vorbestehende chronische Hypertonie. Allerdings gibt es auch Fälle, bei denen nachweislich normotensive Patient/innen plötzlich in einen hypertensiven Notfall geraten. Dies ist zum Beispiel bei akuter Glomerulonephritis oder Präeklampsie der Fall [524-527]. Die pathophysiologischen Mechanismen, die bekanntermaßen auch eine hypertensive endotheliale Schädigung induzieren, sind komplex und bis heute nicht vollkommen geklärt. In jedem Fall finden sich Störungen im Bereich des Renin-Angiotensin-Aldosteron-Systems, in der Freisetzung endogener Vasodilatatoren (insbesondere NO), lokaler Vasokonstriktoren (z. B. Endothelin 1) sowie Adhäsionsmolekülen und inflammatorischer Mediatoren [524-528].

\section{Pathophysiologie des hypertensiven Notfalles}

Üblicherweise wird die Durchblutung der Organe über die Autoregulation bei Blutdruckanstieg reguliert. Hierdurch wird die Gewebeperfusion in einem relativ breiten Blutdruckbereich konstant gehalten. Je schwerer die Hypertonie ist, desto weniger funktioniert jedoch die Autoregulation [524, 529]. Durch Zunahme des Druckes in Arteriolen und Kapillaren kommt es jedoch zunehmend zu einer Schädigung der Gefäßwand mit Ruptur des Gefäßendothels. Im Gehirn führt diese Hyperperfusion mit Versagen der Autoregulation dann letztlich zum Hirnödem als wichtigsten Mechanismus der hypertensiven Encephalopathie. Letztlich ist der BD-Wert, bei dem die oben genannten Mechanismen eintreten, abhängig vom Basisblutdruck. Bei Patient/innen, die bereits eine chronische Hypertonie mit Hypertrophie der Arteriolen haben, wird die Übertragung des Druckes in den Kapillarbereich reduziert. Somit tritt eine maligne Hypertonie bzw. ein hypertensiver Notfall typischerweise erst bei diastolischen BD-Werten über $120 \mathrm{~mm} \mathrm{Hg}$ auf. Andererseits kann jedoch auch bei deutlich niedrigeren diastolischen BD-Werten (z. B. $100 \mathrm{~mm} \mathrm{Hg}$ ) eine hypertensive Encephalopathie bei vorher normotensiven Patient/innen mit akuter hypertensiver Entgleisung auftreten, weil die Autoregulation bereits bei relativ milden hypertensiven BDWerten nicht mehr ausreichend funktioniert [530]. Die Störung der Autoregulation kann zudem dazu führen, dass bei einer gut gemeinten schnellen antihypertensiven Therapie (insbesondere beim Einsatz potenter intravenöser Antihypertensiva) zu schnell die Organperfusion reduziert und damit die Autoregulation der Organe (Gehirn, Herz) überfordert wird und es zu Ischämien kommt [524, 529, 530]. Auch dürfte die pulsatile Belastung (gemessen als Pulsdruck) eine größere Bedeutung als der basale BDWert (der die kontinuierliche Blutdrucklast darstellt) haben, da erstere vermutlich eine engere Beziehung mit der Gefäßschädigung aufweist [531-534].

Bei der Entstehung der fibrinoiden Nekrose der Arteriolen scheint das Renin-Angiotensin-System eine wesentliche Rolle zu spielen. Zumindest konnte im Tierexperiment nachgewiesen werden, dass bei gleichen BD-Werten Tiere, die mit einem ACE-Hemmer (Ramipril) vorbehandelt wurden, ein deutlich geringeres Risiko für einen vaskulären Schaden hatten [528].

\section{Definition und Diagnostik}

Der hypertensive Notfall (englisch: „Hypertensive Emergency“) ist eine Situation, in der eine schwere Hypertonie (meist $\geq 180 / 110 \mathrm{~mm} \mathrm{Hg}$ ) $\mathrm{zu}$ einem akuten HVOS führt, der oft lebensbedrohlich ist und eine sofortige, aber bedachte Intervention erfordert, um den BD zu senken, in der Regel mittels intravenöser Therapie [535]. Die Geschwindigkeit und das Ausmaß des BD-Anstieges sind für den Organschaden 
Tab. 39 Auswahl von klinischen Zuständen, die mit hypertensiven Notfällen assoziiert sein können

\begin{tabular}{l} 
Maligne Hypertonie mit Papillenödem \\
Ischämischer Insult \\
Hämorrhagischer Insult \\
Subarachnoidalblutung \\
Akute Aortendissektion \\
Akute Linksherzinsuffizienz \\
\hline Akutes Koronarsyndrom \\
Akute Glomerulonephritis/rapid progrediente Glomerulonephritis \\
Renale Krise bei Kollagenose /systemischer Sklerose \\
Exzessive Katecholamine (inkl. Krise bei Phäochromozytom) \\
Interaktionen mit MAO-Hemmern \\
Medikamente und Intoxikationen (Amphetamine, Kokain, NSAR, u. a.) \\
Rebound nach abruptem Absetzen älterer Antihypertensiva (z. B. Clonidin) \\
Eklampsie \\
Postoperative Hypertonie \\
Schwere Verbrennungen
\end{tabular}

mindestens so wichtig wie der absolute Wert des BDs [527].

Man unterscheidet u. a. folgende Formen des hypertensiven Notfalles:

1. maligne Hypertonie, charakterisiert durch schwere Hypertonie (in der Regel $\geq 180 / 110 \mathrm{~mm} \mathrm{Hg}$ ) mit fundoskopischen Veränderungen (Einblutung und/ oder Papillenödem), Mikroangiopathie, und intravaskuläre Gerinnung, und evtl. mit Enzephalopathie (in etwa $15 \%$ der Fälle) [536, 537], akuter Herzinsuffizienz und akuter Verschlechterung der Nierenfunktion. Das Kennzeichen dieser Erkrankung ist die fibrinoide Nekrose kleiner Gefäße in Niere, Netzhaut und Gehirn. Der Begriff „maligne“ spiegelt die besonders schlechte Prognose bei NichtBehandlung wider [429, 536-538].

2. Hypertensiver Notfall im Zusammenhang mit anderen klinischen Erkrankungen, die eine dringende Reduktion des BDs erfordern, z. B. akute Aortendissektion, akutes Koronarsyndrom oder akut dekompensierte Herzinsuffizienz.

3. Hypertensiver Notfall bei Phäochromozytom mit Organschäden

4. Hypertensiver Notfall bei schwangeren Frauen mit oder ohne Präeklampsie

Die häufigsten Notfallsymptome hängen von den betroffenen Organen ab. Es können Kopfschmerzen, Sehstörungen, Brustschmerzen, Dyspnoe, Schwindel und andere neurologische Defizite auftreten.

Die hypertensive Enzephalopathie wird durch ein mehr oder weniger ausgeprägtes Hirnödem verursacht, mit langsamem oder auch plötzlichem Auftreten von Kopfschmerzen, Übelkeit, Erbrechen und nicht eindeutig lokalisierbaren neurologischen Symptomen (Somnolenz, Lethargie, Unruhe, Verwirrtheit, Krampfanfällen bis hin zum Koma) [539]. Fokale neurologische Läsionen bzw. Symptome sind selten, dabei sollte an die Differentialdiagnose des zerebralen Insultes gedacht werden.

Ophthalmologische Organmanifestationen können in Form von Retina-Einblutungen oder Retinaexsudaten bis hin zum Papillenödem auftreten und werden mittels Fundusuntersuchung diagnostiziert [524, 527].

Ein akutes Nierenversagen ist gekennzeichnet durch einen akuten Kreatininanstieg und ist ein Hinweis für eine maligne Nephrosklerose, die zu renaler Schädigung, Hämaturie und Proteinurie führen kann. Die Nierenbiopsie bei maligner Nephrosklerose zeigt eine fibrinoide Nekrose der Arteriolen und Kapillaren, die ähnlich einem hämolytisch-urämischen Syndrom imponieren können. Diese akute renale Schädigung führt zu einer glomerulären Ischämie mit einer ausgeprägten reninabhängigen zusätzlichen Hypertoniekomponente, die den BD-Anstieg bis hin zum Vollbild der malignen Hypertonie verstärken kann [524, 527, 528].

Tab. 39 fasst die häufigsten Erkrankungen, die mit einem hypertensiven Notfall einhergehen, zusammen.

Die Hypertensive Krise (englisch: „Hypertensive Urgency") bezeichnet eine schwere arterielle Hypertonie bei Patient/innen, die in einer Notfallabteilung ohne klinische Hinweise auf HVOS vorstellig werden [52]. Bei diesen Patient/innen sollte ebenfalls der BD gesenkt werden, in erster Linie mit oralen Medikamenten. Eine stationäre Aufnahme ist in der Regel nicht notwendig. Diese Patient/innen sollten jedoch auf jeden Fall ambulant nachkontrolliert werden, um sicherzustellen, dass der BD im weiteren Verlauf wieder im Zielbereich ist, da bekannt ist, dass das kardiovaskuläre Risiko dieser Patient/innen erhöht ist [523].

Akute und starke Erhöhungen des BDs können auch durch Einnahme von Medikamenten oder Drogen entstehen, wie z. B., Sympathomimetika (MetaAmphetamin, Kokain), aber auch durch nicht-steroidale Antirheumatika [76]. Besonders häufig führt die Einnahme von nicht-steroidalen Antirheumatika oder von anderen Substanzen, die entweder per se zu einer BD-Steigerung führen oder die Wirkung von Antihypertensiva abschwächen, zu einem hypertensiven Notfall oder einer hypertensiven Entgleisung (Krise). Daher ist es außerordentlich wichtig, die Patient/ innen nach Schmerzen bzw. Einnahme von Schmerzmitteln zu fragen. Wenn eine hypertensive Krise oder Notfall durch die Einnahme von nicht-steroidalen Antirheumatika bedingt ist, sollten in diesem Fall bevorzugt Kalziumantagonisten eingesetzt werden, die in dieser Situation wahrscheinlich am besten wirken [540, 541].

Auch Schmerzen, Angst und andere Beschwerden können bei Patient/innen in einer Notaufnahme zur starken Erhöhung des BDs führen. Hier empfiehlt es sich, zunächst die primäre Ursache zu behandeln (z. B. Schmerztherapie, Therapie des psychischen Ausnahmezustandes, Blasenentleerung bei übervoller Blase u.a.). Hierdurch normalisiert sich der BD häu- 
Tab. 40 Behandlungs-Optionen für spezifische hypertensive Notfälle [52, 498, 543, 545]

\begin{tabular}{|c|c|c|c|}
\hline Klinik & Zeit-Horizont und Blutdruck-Ziel & First-line-Therapie & Alternativen \\
\hline $\begin{array}{l}\text { Maligne Hypertonie mit oder } \\
\text { ohne akutem Nierenversagen }\end{array}$ & $\begin{array}{l}\text { Senkung des arteriellen Mitteldrucks um 20-25\% innerhalb } \\
\text { mehrerer Stunden }\end{array}$ & Labetalol, Nicardipin & Urapidil, Nitroprussid \\
\hline Hypertensive Enzephalopathie & Sofortige Senkung des arteriellen Mitteldrucks um 20-25\% & Labetalol, Nicardipin & Urapidil, Nitroprussid \\
\hline Akutes Koronarsyndrom & $\begin{array}{l}\text { Sofortige Senkung des systolischen Blutdrucks unter } \\
140 \mathrm{~mm} \mathrm{Hg}\end{array}$ & Nitroglycerin, Labetalol & Urapidil \\
\hline $\begin{array}{l}\text { Akutes kardiogenes Lungen- } \\
\text { ödem }\end{array}$ & $\begin{array}{l}\text { Sofortige Senkung des systolischen Blutdrucks unter } \\
140 \mathrm{~mm} \mathrm{Hg}\end{array}$ & Nitrate mit Schleifendiuretika & Urapidil mit Schleifendiuretika \\
\hline Akute Aortendissektion & $\begin{array}{l}\text { Sofortige Senkung des systolischen Blutdrucks unter } \\
120 \mathrm{~mm} \mathrm{Hg} \text { und der Herzfrequenz unter } 60 / \mathrm{min}\end{array}$ & $\begin{array}{l}\text { Esmolol und Nitroglycerin } \\
\text { oder Nitroprussid, Nicardipin }\end{array}$ & Labetalol oder Metoprolol \\
\hline $\begin{array}{l}\text { Eklampsie, schwere Präek- } \\
\text { lampsie/HELLP }\end{array}$ & $\begin{array}{l}\text { Sofortige Senkung des systolischen Blutdrucks unter } \\
160 \mathrm{~mm} \mathrm{Hg} \text { und des diastolischen Blutdrucks unter } \\
105 \mathrm{~mm} \mathrm{Hg}\end{array}$ & $\begin{array}{l}\text { Labetalol, } \\
\text { Urapidil, Nicardipin, Magnesi- } \\
\text { um-Sulfat, } \\
\text { Nitroglycerin i.v. (bei Lungen- } \\
\text { ödem) }\end{array}$ & $\begin{array}{l}\text { Frühzeitige Entbindung erwä- } \\
\text { gen }\end{array}$ \\
\hline
\end{tabular}

fig, sodass nur in Ausnahmefällen eine spezifische antihypertensive Therapie notwendig ist.

Bei hypertensivem Notfall werden die in Tab. 40 dargestellten Untersuchungen empfohlen.

\section{Fact Box: Diagnostisches Workup bei Verdacht auf hypertensiven Notfall}

Primäres Screening:

- Fundoskopie

- 12-Kanal-EKG

- Hämoglobin, Thrombozyten, Fibrinogen

- Kreatinin im Serum, eGFR, Elektrolyte, LDH, Haptoglobin

- Albumin: Kreatinin im Harn, Erythrozyten-Morphologie im Harn, Leukozyten

- Schwangerschaftstest bei Frauen im gebärfähigen Alter

\section{Spezifische Tests nach Indikation:}

- Troponin (bei Verdacht auf akutes Koronarsyndrom, z. B. akute Brustschmerzen)

- Nt-proBNP bei Verdacht auf akute Herzinsuffizienz

- Thorax-Röntgen

- Echokardiographie (Aortendissektion, Herzinsuffizienz oder Ischämiezeichen)

- CT der Aorta bei V.a. Aortendissektion/Aneurysma

- CT oder MRT-Gehirn (Beteiligung des ZNS)

- Abdomen-Sonographie (Nierenschädigung. V.a. Nierenarterienstenose)

- Drogentest im Harn (insb. Methamphetamine, Kokain)

\section{Therapie}

Zur Therapie von hypertensiven Notfällen gibt es wenige kontrollierte Studien. Die nationalen und internationalen Empfehlungen beruhen daher nicht auf guten kontrollierten, randomisierten Studien, sondern auf einer breiten international anerkannten
Expertenmeinung [52]. Die Mortalität bei unbehandelten hypertensiven Notfällen und auch die Komplikationsrate bei diesen Patient/innen ist sehr hoch [522]. Tab. 40 fasst mögliche therapeutische Strategien zusammen.

Die wichtigsten Überlegungen bei der Festlegung der Behandlungsstrategie sind:

1. Die Identifizierung der betroffenen Zielorgane, zur Klärung ob andere therapeutische Maßnahmen zusätzlich zur BD-Senkung nötig sind und ob es eine auslösende Ursache für den akuten BD-Anstieg gibt, der die Therapie beeinflussen könnte (z. B. Schwangerschaft, medikamentöse Ursachen);

2. Der empfohlene Zeitraum und die Größenordnung der BD-Senkung, die für eine sichere Behandlung erforderlich ist;

3. Die Art der BD-Senkung, die bei einem hypertensiven Notfall in der Regel mittels intravenöser Therapie mit einem Medikament mit kurzer Halbwertszeit erfolgt, um eine sorgfältige Titration der BDSenkung unter engmaschiger Überwachung zu ermöglichen.

Patient/innen mit einem hypertensiven Notfall sollten unverzüglich auf einer Überwachungsstation (Intermediate Care Unit - IMCU) behandelt werden. Eine intravenöse Therapie wird primär für die Behandlung hypertensiver Notfälle empfohlen. Bei maligner Hypertonie ist eine orale Therapie mit ACE-Hemmern, ARBs und Beta-Blockern manchmal sehr effektiv, da es in diesen Situationen zu einer massiven Aktivierung des Renin-Systems durch die renale Ischämie kommt. Allerdings sollten geringe Anfangsdosen verwendet werden, da diese Patient/innen sehr empfindlich auf diese Mittel reagieren können, die Behandlung sollte im Krankenhaus stattfinden [535].

Wie stark der BD gesenkt wird, hängt wesentlich von der jeweiligen Erkrankung ab. Bei akutem ischämischen Insult sollte eine Blutdrucksenkung nur bei geplanter Thrombolyse oder sehr hohen Blutdruckwerten $(>220 / 120 \mathrm{~mm} \mathrm{Hg}$ ) erfolgen (siehe Abschn. 17), weil die bisher durchgeführten Studien 
Tab. 41 Empfohlene Pharmaka und ihre Wirkung

\begin{tabular}{|c|c|c|c|c|c|}
\hline Medikament & $\begin{array}{l}\text { Wirkungs- } \\
\text { eintritt }\end{array}$ & $\begin{array}{l}\text { Wirkungs- } \\
\text { dauer }\end{array}$ & Dosis & Kontraindikationen & Nebenwirkungen \\
\hline Urapidil & $3-5 \min$ & $4-6 \mathrm{~h}$ & $\begin{array}{l}12,5-25 \mathrm{mg} \text { i.v. Bolus, } \\
\text { Perfusor } 5-40 \mathrm{mg} / \mathrm{h}\end{array}$ & Keine & $\begin{array}{l}\text { Selten: Übelkeit, Kopfschmerzen, } \\
\text { Schwindel }\end{array}$ \\
\hline Esmolol & $1-2 \mathrm{~min}$ & $10-30 \mathrm{~min}$ & $\begin{array}{l}0,5-1 \mathrm{mg} / \mathrm{kg} \text { Bolus, } \\
\text { Perfusor } 50-300 \mu \mathrm{g} / \mathrm{kg} / \mathrm{min}\end{array}$ & $\begin{array}{l}\text { AV-Blockierungen, akut dekompen- } \\
\text { sierte Herzinsuffizienz, Asthma, } \\
\text { Bradykardie }\end{array}$ & $\begin{array}{l}\text { Bradykardie } \\
\text { CAVE: Nekrosen bei Paravasat! }\end{array}$ \\
\hline Metoprolol & $1-2 \min$ & $5-8 \mathrm{~h}$ & $\begin{array}{l}2,5-5 \mathrm{mg} \text { Bolus iv., ggf. alle } \\
5 \mathrm{~min} \text { wiederholen, max. Dosis } \\
15 \mathrm{mg}\end{array}$ & $\begin{array}{l}\text { AV-Blockierungen, akut dekompen- } \\
\text { sierte Herzinsuffizienz, Asthma, } \\
\text { Bradykardie }\end{array}$ & Bradykardie \\
\hline Nicardipin & $5-15 \min$ & $3 B-40 \mathrm{~min}$ & $5-15 \mathrm{mg} / \mathrm{h}$ iv & Akutes Leberversagen & Kopfschmerzen, Reflextachykardie \\
\hline $\begin{array}{l}\text { Glycerol-trinitrat } \\
\text { (Nitroglycerin) }\end{array}$ & $1-2 \mathrm{~min}$ & $3 \min$ & $\begin{array}{l}0,1-0,5 \mathrm{mg} \mathrm{s} . \mathrm{l} . \text {, dann } \\
\text { 5-200 } \mathrm{gg} / \mathrm{min}(=0,3-12 \mathrm{mg} / \mathrm{h}) \\
\text { i.v. }\end{array}$ & $\begin{array}{l}\text { Erhöhter Hirndruck, akuter Hinter- } \\
\text { wand-STEMI, Hypovolämie, HOCM }\end{array}$ & $\begin{array}{l}\text { Cephalea, Toleranz, Hirndrucksteige- } \\
\text { rung } \\
\text { Reflextachykardie }\end{array}$ \\
\hline Nitroprussid & Sofort & $1-2 \min$ & $0,3-10 \mu \mathrm{g} / \mathrm{kg} / \mathrm{min}$ & $\begin{array}{l}\text { Akutes Leberversagen, akutes Nie- } \\
\text { renversagen }\end{array}$ & $\begin{array}{l}\text { Cynaid-Intoxikation, Muskelzittern, } \\
\text { Übelkeit }\end{array}$ \\
\hline Enalaprilat & $5-15 \mathrm{~min}$ & $4-6$ Std & $0,625-1,25 \mathrm{mg}$ iv. Bolus & Anamnestisch Angioödem & - \\
\hline Clonidin & $30 \mathrm{~min}$ & $4-6 \mathrm{~h}$ & $\begin{array}{l}150-300 \mu \mathrm{g} \text { i.v. Bolus über } \\
5-10 \mathrm{~min} \\
\text { Perfusor: } 0,015-0,03 \mathrm{mg} / \mathrm{h}\end{array}$ & AV-Block II/III & Mundtrockenheit, Sedierung \\
\hline $\begin{array}{l}\text { Nifedipin (z. B. } \\
\text { Adalat) }\end{array}$ & $1-2 \min$ & $2-10 \min$ & $0,63-1,25 \mathrm{mg} / \mathrm{h}$ & CAVE lichtempflindliche Lösung! & $\begin{array}{l}\text { Reservemittel bei schwerer Hyperto- } \\
\text { nie, Kopfschmerzen, Reflextachykar- } \\
\text { die }\end{array}$ \\
\hline
\end{tabular}

keinen klinischen Nutzen einer stärkeren Blutdrucksenkung gezeigt haben. Bei akutem Lungenödem und Aortendissektion hingegen muss eine rasche und aggressive Blutdrucksenkung erfolgen (Tab. 40).

Aufgrund der Verfügbarkeit und Erfahrungen in Österreich empfehlen wir als Mittel erster Wahl in den meisten Fällen Urapidil intravenös (12,5 mg als Bolus oder als Kurzinfusion), welches nach 15-20 min ggf. wiederholt werden kann [521, 542, 543]. Bei pectanginösen Beschwerden sollte Nitroglycerin via Perfusor verabreicht werden (insbesondere bei akutem Koronarsyndrom und Lungenödem). Clonidin kann ebenfalls in intravenöser Form verwendet werden (vorteilhaft auch bei gleichzeitig bestehender Agitiertheit, Unruhe oder Alkoholentzugssyndrom). Bei sehr schweren oder therapieresistenten Fällen ist die Gabe von Nitroprussid-Natrium über Perfusor möglich. Bei therapieresistenten hypertensiven Notfällen kann die kontinuierliche i.v. Gabe von Nifedipin über Perfusor verwendet werden. Auch die Gabe des Betablockers Esmolol über Perfusor wird empfohlen [100, 520].

Die internationalen Empfehlungen für die bei hypertensivem Notfall (siehe Tab. 41) zu verwendenden Pharmaka beinhalten Labetalol, Nicardipin, Nitroglycerin, Furosemid, Urapidil, Clonidin und Natriumnitroprussid [52, 520, 524, 527, 539, 544].

Die Dosierungen, sowie die Vor- und Nachteile der verschiedenen Medikamente sind in Tab. 41 angegeben.

Eine nicht indizierte und unkontrollierte BD-Senkung ist nicht empfohlen, da dies zu Komplikationen führen kann [76, 535, 546, 547]. Abzuraten ist insbesondere von der sublingualen Applikation von unretardierten Kalziumantagonisten (wie zum Beispiel Nifedipin) in Form von Tropfen, Sprays, Phiolen o. ä., wegen der nachweislich schlechten Steuerbarkeit, der massiven Sympathikusaktivierung und der Gefahr eines $\mathrm{zu}$ raschen und unkontrollierbaren BD-Abfalles mit der Folge zerebraler und kardialer Ischämien [520, 546, 548-551].

Im Gegensatz dazu ist bei der hypertensiven Krise keine Notfalltherapie indiziert. Der Beruhigung des Patienten/der Patientin kommt eine wesentliche Rolle zu. Ausreichend ist oft, die Medikation fortzusetzen bzw. auslösende Ursachen (wie z. B. Einnahme von BD-steigernden Medikamenten) abzustellen. Ein intensives Schulungsprogramm wie z. B. das steirische „herz.leben“ Projekt, kann nachweislich die Blutdruckeinstellung verbessern [552]. Therapeutisch reicht bei der hypertensiven Entgleisung (Krise) meist, Patient/innen auf die Notwendigkeit der regelmäßigen Medikamenteneinnahme hinzuweisen. Gegebenenfalls muss auch die antihypertensive Therapie intensiviert bzw. umgestellt werden. Relevant erscheint als Compliance fördernde Maßnahme auch, die Ängste von Patient/innen vor Nebenwirkungen abzubauen, was durch eine Verstärkung der Arzt/Ärztin-Patient/innen-Beziehung und das Vertrauensverhältnis wesentlich unterstützt wird. Dieses ist besonders wichtig, da die Patientin/der Patient häufig die Medikamente aufgrund von Nebenwirkungen oder der Furcht vor möglichen Nebenwirkungen nicht einnimmt, über die Nebenwirkungen aber nicht unbedingt aktiv von sich aus spricht [100, 520].

\section{Prognose und Nachsorge}

Das Überleben von Patient/innen mit hypertensiver Krise hat sich in den vergangenen Jahrzehnten dramatisch verbessert [553]. Sie sind aber nach wie vor 
einem hohen Risiko ausgesetzt und sollten ggf. je nach Klinik auf das Vorliegen einer sekundäre Hypertonie untersucht werden. Nach der Entlassung aus dem Krankenhaus, wenn der BD unter oraler Therapie ein sicheres und stabiles Niveau erreicht hat, sollten regelmäßige, mindestens monatliche ambulante Kontrollen bei niedergelassenen Ärztinnen und Ärzten und einer Hypertonie-Ambulanz erfolgen, bis das optimale BD-Ziel erreicht ist [52].

\section{Perioperatives Blutdruckmanagement}

Mit der stetig steigenden Anzahl von hypertensiven Patientinnen und Patienten, die sich chirurgischen Eingriffen unterziehen, ist das perioperative Management der Bluthochdruckkrankheit ein häufiges Problem.

Während eine moderate Blutdruckerhöhung per se keinen ausgeprägten Risikofaktor für kardiovaskuläre Komplikationen bei nicht-kardiochirurgischen Eingriffen darstellt, ist das Feststellen des kardiovaskulären Gesamtrisikos einschließlich des Risikofaktors arterielle Hypertonie und der Beurteilung Hypertonievermittelter Organschäden sowohl bei behandelten als auch bei unbehandelten Bluthochdruck-Patient/ innen von entscheidender Bedeutung [554].

Bei Patientinnen und Patienten mit isolierter systolischer Hypertonie, die sich einer aortokoronaren Bypassoperation unterziehen, konnte eine Zunahme der kardiovaskulären Morbidität nachgewiesen werden.

Ein Absetzen notwendiger chirurgischer Eingriffe ist bei Patient/innen mit erst- oder zweitgradiger Hypertonie üblicherweise nicht erforderlich, während bei Patient/innen mit einem systolischen $\mathrm{BD}$ über $180 \mathrm{~mm} \mathrm{Hg}$ und einem diastolischen $\mathrm{BD}$ über $110 \mathrm{~mm} \mathrm{Hg}$ die Verschiebung des chirurgischen Eingriffes bis zur Kontrolle des BDs sinnvoll erscheint, sofern keine chirurgische Notfallsituation vorliegt.

Die Vermeidung großer perioperativer Blutdruckschwankungen scheint wichtig zu sein [554].

Eine rezente randomisierte Studie zeigte, dass bei Patient/innen, die sich einem abdominalchirurgischen Eingriff unterzogen haben, ein Blutdruckniveau mit einer Schwankungsbreite von $10 \%$ im Vergleich zur präoperativen Arztmessung in einem reduzierten Risiko von postoperativer Organdysfunktion resultiert [555].

Es besteht keine klare Evidenz für oder gegen eine bestimmte antihypertensive Therapiestrategie bei Patient/innen, die sich einem nicht-kardiochirurgischen Eingriff unterziehen. Somit unterliegen diese Patient/innen den allgemeinen Behandlungsalgorithmen [556, 557].

Die perioperative Verwendung eines Betablockers wurde in den letzten Jahren kontroversiell diskutiert. Bedenken zur Sicherheit der Anwendung von Betablocker im perioperativen Setting wurden im Rahmen einer rezenten Metaanalyse geäußert, die ein erhöhtes Risiko für Hypotension, Schlaganfälle und Mortalität bei Patient/innen unter perioperativer Betablockade im Vergleich zu Placebo zeigte [554, 556-559].

Dennoch ist die kontinuierliche Gabe des Betablockers in der perioperativen Phase bei hypertensiven Patient/innen unter einer chronischen Betablocker-Therapie empfohlen, zumal das plötzliche Absetzen des Betablockers zu einem Rebound-Effekt mit Anstieg von BD und Herzfrequenz führen kann [554, 558-565]. Ähnliche Effekte sind bei plötzlichem Absetzen zentral wirksamer Substanzen wie Clonidin zu erwarten und sollten vermieden werden.

In mehreren rezenten Publikationen wurden die Effekte einer perioperativen Gabe von ACE-Hemmern und ARBs auf die Blutdruckregulation intraoperativ diskutiert [554]. Das Absetzen von ACE-Hemmern oder ARB's reduzierte das Risiko für intraoperative Hypotensionen [566]. Die perioperative Unterbrechung einer Therapie mit ACE-Hemmern/ARBs, $24 \mathrm{~h}$ vor nicht-kardialer Chirurgie, wurde rezent in einer heterogenen Patient/innengruppe untersucht und führte zu einer signifikanten Reduktion kardiovaskulärer Ereignisse und der 30-Tage-Mortalität nach dem chirurgischen Eingriff [567].

\section{Fact Box Bluthochdruck und geplante Operation}

- Neu-diagnostizierte Bluthochdruckpatient/innen sollten vor elektiven chirurgischen Eingriffen präoperativ auf Hypertonie-assoziierte Organschäden und das gesamte kardiovaskuläre Risiko untersucht werden.

- Starke Blutdruckschwankungen während der perioperativen Phase sollten vermieden werden.

- Nicht-kardiochirurgische Eingriffe müssen bei Patient/innen mit einem Blutdruck unter $180 \mathrm{~mm} \mathrm{Hg}$ systolisch und unter $110 \mathrm{~mm} \mathrm{Hg}$ diastolisch nicht verschoben werden.

- Perioperativ sollte eine kontinuierliche BetablockerTherapie bei Patient/innen mit arterieller Hypertonie fortgesetzt werden.

- Das abrupte Unterbrechen einer Betablocker-Therapie oder einer Therapie mit zentral wirksamen Substanzen (Clonidin), ist möglicherweise mit negativen kardiovaskulären Effekten und mit einer erhöhten Ereignisrate assoziiert und wird nicht empfohlen.

- Betablocker sollten bei stabilen Patient/innen nicht am Tag des chirurgischen Eingriffes begonnen werden.

- Transiente präoperative Unterbrechung einer Therapie mit ACE-Hemmern oder ARBs sollte bei Patient/ innen mit arterieller Hypertonie, die sich einem nichtkardiochirurgischen Eingriff unterziehen, in Betracht gezogen werden.

\section{Peri- und intraoperatives Blutdruckmanagement}

Etwa $25 \%$ der Patient/innen, die sich einem großen nicht-kardialen chirurgischen Eingriff unterziehen und etwa $80 \%$ der Patient/innen, die herzchirurgisch behandelt werden, haben im perioperativen Setting 
zumindest eine hypertensive Episode. Bluthochdruck in der perioperativen Phase erhöht das Risiko für kardiovaskuläre und zerebrale Ereignisse und Blutungskomplikationen [568]. Die Häufigkeit und der Schweregrad dieser Episoden sind vom Schweregrad der vorbestehenden Hypertonie abhängig.

Intraoperative hypertensive Entgleisungen werden häufig während der Anästhesieeinleitung und während der Intubation beobachtet.

Außerordentlich starke BD-Steigerungen treten häufig bei Patient/innen mit schlecht kontrollierter Bluthochdruckkrankheit auf.

Unter intensivierter Anästhesie und damit assoziierter Sympathikushemmung und Verlust der Barorezeptorenkontrolle kann in weiterer Folge häufig eine intraoperative Hypotonie beobachtet werden. Im Allgemeinen kann eine Labilität der Blutdruckregulation im intraoperativen Setting häufiger bei Patient/innen mit schlecht kontrollierter arterieller Hypertonie beobachtet werden, während Patient/innen mit guter Blutdruckkontrolle im intraoperativen Setting ähnlich wie normotensive Patient/innen reagieren.

Es liegen keine qualitativ hochwertigen randomisierten Studien bzgl. der Behandlung des Bluthochdrucks bei Patient/innen, die sich einem großen chirurgischen Eingriff unterziehen, vor.

Im Rahmen des Managements von Patient/innen mit perioperativer Hypertonie ist es erforderlich, potenzielle Zusatzfaktoren wie etwa Überwässerung, ungenügende Schmerzkontrolle, Oxygenierungsprobleme und Blasenentleerungsstörung $\mathrm{zu}$ beachten und gegebenenfalls zu korrigieren.

Für das intravenöse antihypertensive medikamentöse Management, zur Behandlung hypertensiver Episoden im perioperativen Setting (perioperative Hypertonie mit Blutdruckwerten über 160/90 mm Hg oder systolischer BD-Erhöhung über $20 \%$ der präoperativen Werte, die über mehr als 15 min persistieren), werden Nitroglycerin, Urapidil, Nicardipin, Clevidipin und Esmolol empfohlen, bis die orale Medikation wieder eingeleitet werden kann [569-574].

\section{Resistente Hypertonie}

Von resistenter Hypertonie spricht man bei fehlender Blutdrucksenkung unter $140 \mathrm{~mm} \mathrm{Hg}$ systolisch und/oder $90 \mathrm{~mm} \mathrm{Hg}$ diastolisch (Office-BD) trotz maximal verträglicher Therapie, zumindest aber einer 3-fach Kombination aus Diuretikum und 2 weiteren Substanzklassen (typischerweise ACE-Hemmer/ARB und Kalziumantagonist) in adäquater Dosierung [52]. Die Prävalenz der therapieresistenten Hypertonie wird variierend mit 3-30\% beschrieben [575]. Die Betreuung dieser Patient/innen ist herausfordernd, zeitaufwendig und häufig auch frustrierend, sodass die frühe Überweisung an ein Zentrum zu empfehlen ist. Bei Vorliegen einer Therapieresistenz ist mit vermehrten kardiovaskulären Ereignissen zu rechnen [576]. Dies trifft besonders auf Patient/innen mit der seltenen refraktären Hypertonie [577] zu, welche als Nicht-Erreichen der Zielwerte trotz antihypertensiver Fünffach-Kombination definiert ist. Dabei muss die Therapie ein langwirksames Thiazid/thiazidähnliches Diuretikum (z.B. Chlorthalidon) und Spironolacton beinhalten.

Die Diagnose resistente Hypertonie muss durch ein 24-h-Blutdruckmonitoring (nur wenn dies nicht möglich ist, zumindest durch mehrfache BD-Selbstmessungen nach dem standardisierten Schema aus Abschn. 3) bestätigt werden. So kann einerseits eine Weißkittelhypertonie ausgeschlossen und mögliche Hinweise auf eine sekundäre Hypertonie - wie fehlende Nachtabsenkung - detektiert werden. Ein besonderes Augenmerk muss sowohl in der Ordinations-, als auch in der Selbstmessung auf die richtige Durchführung der BD-Messung und die Verwendung validierter Geräte gelegt werden (siehe Abschn. 3). Für alle Messmethoden gilt die Beachtung der richtigen Manschettengröße, da eine zu kleine Manschette zur Überschätzung des BDs um bis zu $15 \mathrm{~mm} \mathrm{Hg}$ führen kann [578]. Ist das Vorliegen einer arteriellen Hypertonie mittels 24-h-Blutdruckmonitoring (bevorzugt!) oder Blutdruckselbstmessung bestätigt, gilt es, zunächst eine Pseudoresistenz, und falls diese nicht vorliegt, eine sekundäre Hypertonie auszuschließen (Abb. 8). Fehlende Adhärenz ist eine der Hauptursachen für eine vermeintlich „resistente“ Hypertonie und wird in Untersuchungen, in denen die Therapietreue durch Urinanalysen überprüft wurde, mit bis zu $50 \%$ beschrieben [579]. Die Einnahme blutdrucksteigernder Medikamente und Drogen (z. B. nichtsteroidale Antirheumatika, Steroide, Sympathikomometika, Kokain, etc, siehe Abschn. 27) sowie ein inadäquates Therapieregime durch „physicians inertia“ sind ebenfalls häufige Ursachen für Pseudoresistenz.

Häufige Ursachen einer sekundären (und therapieresistenten) Hypertonie sind Hyperaldosteronismus, obstruktive Schlafapnoe (meist, aber nicht immer mit Adipositas assoziiert), Nierenarterienstenose und chronische Nierenerkrankungen. Auch starke Gewichtszunahme, exzessiver Alkoholkonsum, übermäßige Kochsalzzufuhr und Hypertonie-vermittelte Organschäden (insbesondere chronische Niereninsuffizienz) führen zu schwer therapierbarem arterielle Hypertonus. Eine erhöhte arterielle Gefäßsteifigkeit sollte nicht außer Acht gelassen werden, da eine resistente Hypertonie häufig $\mathrm{zu}$ einem vaskulären Remodeling führt. Die Testung kann sehr einfach mittels einer Messung der Carotis-Femoralis-Pulswellengeschwindigkeit, dem Goldstandard-Verfahren [580], oder der Berechnung des Pulsdruckes (pulse pressure, PP) erfolgen. Bei der Pulswellengeschwindigkeit (pulse wave velocity, PWV) gilt ein Grenzwert von $>10 \mathrm{~m} / \mathrm{s}$ [63], beim Pulsdruck von $60 \mathrm{~mm} \mathrm{Hg} \mathrm{(Abschn.} \mathrm{6).}$ 
Abb. 8 Diagnosealgorithmus therapieresistente $\mathrm{Hy}-$ pertonie

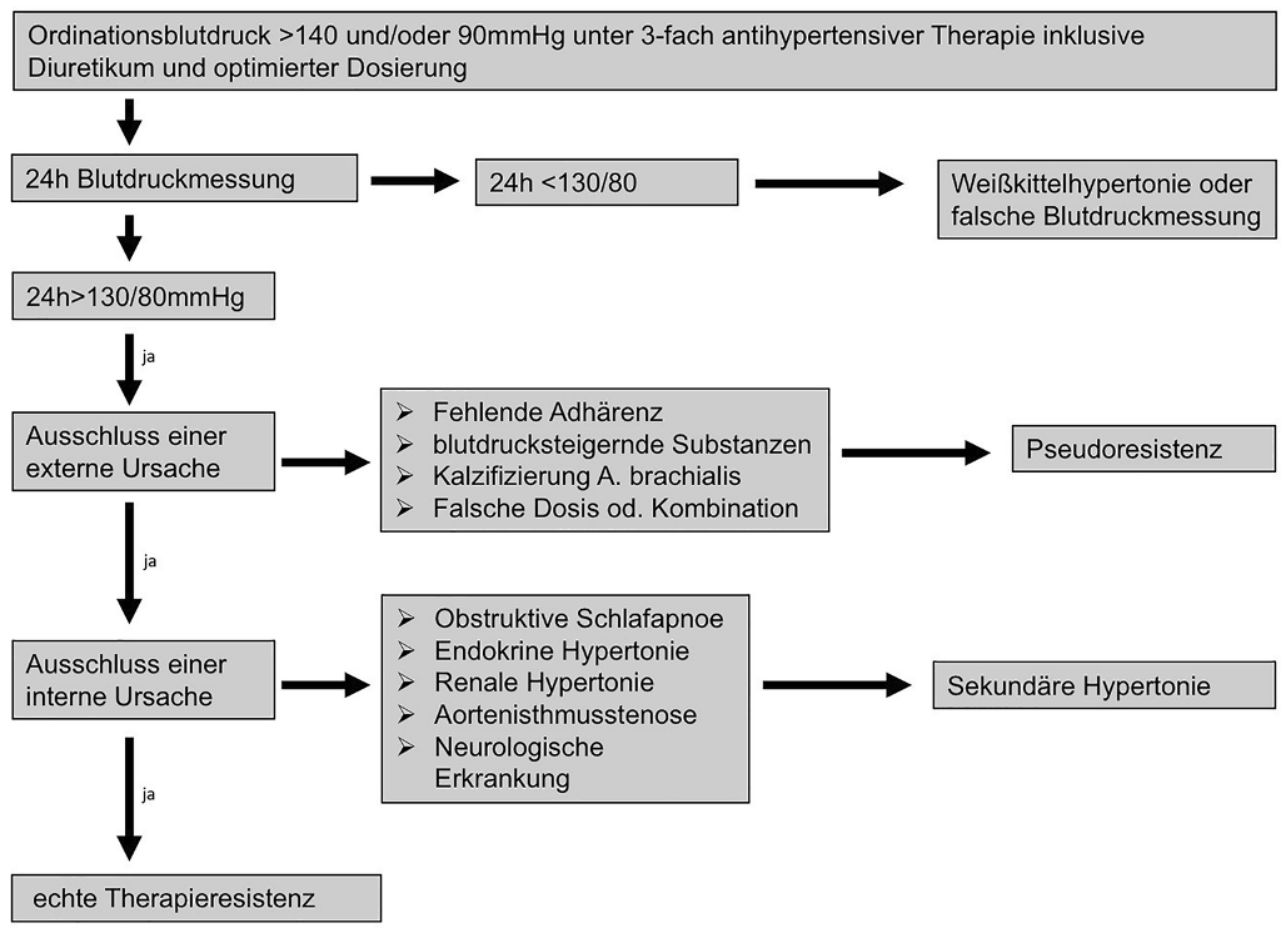

\section{Therapeutische Ansätze bei resistenter Hypertonie}

Grundlage bilden Lebensstilmaßnahmen, vor allem eine konsequente Salzrestriktion, weiters eine Beendigung blutdrucksteigernder Medikamente sowie eine Optimierung der Adhärenz (siehe Abschn. 25).

In der PATHWAY-2-Studie [225] war Spironolacton (25-50 mg/Tag) die wirksamste antihypertensive Substanz als Zusatz zur Standard-Dreifachkombination ACE-Hemmer/ARB + Kalziumantagonist + Diuretikum. Dies hat sich auch in Meta-Analysen bestätigt [581]. Untersuchungen an adipösen Hochdruckpatient/ innen mit Spironolacton zeigten besonders in Kombination mit Thiaziden gute blutdrucksenkende Effekte, was auf eine vermehrte Aldosteronausschüttung bei zunehmendem Körpergewicht zurückgeführt wird [582]. Spironolacton weist allerdings ein beträchtliches Nebenwirkungsprofil auf, das neben einer Hyperkaliämie das Auftreten von hormonellen Störungen (Gynäkomastie, Menstruationsstörungen und Impotenz) umfasst. In letzterem Fall könnten Eplerenon (50-100 mg tgl) oder auch Amilorid (10-20 mg tgl) [132] versucht werden. Beide sind aber ebenso wie Spironolacton bei Nierenfunktioneinschränkung (GFR $<30 \mathrm{ml} / \mathrm{min}$ ) und bei Hyperkaliämie kontraindiziert.

Auch Betablocker (Bisoprolol) und Alphablocker (Doxazosin) bewirkten in der PATHWAY-2-Studie [225] als Zusatz zur Dreifachkombination (s. oben) eine stärkere BD-Senkung als Placebo und haben somit eine gewisse Evidenz, besonders wenn Spironolacton kontraindiziert ist oder nicht vertragen wird.

Aus pathophysiologischer Sicht kann man hinsichtlich der medikamentösen 3-fach Therapie mit ACE-
Hemmer/ARB + Calciumantagonist + Diuretikum argumentieren, dass ältere Patient/innen häufig einen niedrigen Reninstatus haben und daher schlechter auf ACE-Hemmer/ARBs ansprechen [583]. In Ermangelung randomisierter Studien zu Vielfachkombinationen empfehlen wir folgende klinische Überlegungen: Zunächst sollte eine klinische Evaluierung hinsichtlich Salz- und Volumenstatus (periphere Ödeme, erhöhte Natriumausscheidung, erhöhte linksventrikuläre Füllungsdrücke), erhöhter sympathischer Aktivität (erhöhte Herzfrequenz in der 24-h-Messung), und erhöhter Gefäßsteifigkeit (erhöhte PWV oder PP) erfolgen. Ist die Volumsexpansion schon klinisch vorherrschend, sind Diuretika (Spironolacton, bei Unverträglichkeit Eplerenon oder Amilorid, auch in Kombination mit höher dosiertem Thiazid bzw. thiazidartigem Diuretikum oder Schleifendiuretikum) empfehlenswert. Hier ist oft eine sequentielle Nephronblockade erforderlich. Bei Patient/innen mit eingeschränkter Nierenfunktion, insbesondere bei einer GFR $<30 \mathrm{ml} / \mathrm{min}$, wirken Thiaziddiuretika schlechter, daher muss häufig auf ein Schleifendiuretikum gewechselt werden bzw eines dazukombinert werden. Liegt eine sympathische Überaktivierung vor, können ein Alpha-oder Betablocker (z.B. Doxazosin, Carvedilol, Bisoprolol, Nebivolol) bzw. ein zentral wirksames Sympatholytikum (z. B. Moxonidin oder Rilmenidin) versucht werden. Direkte Vasodilatatoren wie Hydralazin und Minoxidil können in schweren Fällen eingesetzt werden, müssen aber engmaschig kontrolliert werden, da sie zu schwerwiegenden $\mathrm{Ne}$ benwirkungen führen können, insbesonders zu einer Volumsretention. 
Interventionelle Verfahren zur BD-Senkung können für einzelne gut abgeklärte Patient/innen mit resistenter Hypertonie Erfolge bringen, sind derzeit aber nicht als Standardtherapie empfohlen (siehe Abschn. 24). An dieser Stelle soll jedoch darauf hingewiesen werden, dass die Carotisstimulation bei hohen Konzentrationen von Angiotensin II oder Aldosteron wenig Effekt zeigte [584]. Die renale Sympathikusdenervierung hatte bei Patient/innen mit erhöhter arterieller Gefäßsteifigkeit [585] und/oder isolierter systolischer Hypertonie [586] keine oder nur geringe BDSenkung zur Folge. Es wird empfohlen, Patient/innen mit therapieresistenter arterieller Hypertonie bei Diagnosestellung hinsichtlich eines bestehenden Hypertonie-vermittelten Organschadens abzuklären und regelmässig zu kontrollieren.

\section{Interventionelle Verfahren zur Blutdrucksen- kung}

Wie in Abschn. 23 ausgeführt, werden Patient/innen, deren Office-BD sich trotz einer antihypertensiven 3-fach-Kombinationstherapie einschließlich eines Diuretikums nicht unter $140 / 90 \mathrm{mmHg}$ senken lässt, als therapieresistent bezeichnet [52]. Sie haben ein besonders hohes kardiovaskuläres Risiko [587]. Für solche Patient/innen mit therapie-resistenter Hypertonie (RH) sind innerhalb der letzten Jahre zahlreiche interventionelle oder Device-gestützte Therapien vorgestellt worden, um eine Verbesserung der Blutdruckkontrolle zu ermöglichen. Das therapeutische Target dieser Verfahren liegt in einer Beeinflussung der bei $\mathrm{RH}$ vorhandenen autonomen Dysbalance, entweder durch Reduktion der Aktivität des sympathischen Nervensystems oder einer verstärkten Aktivierung des parasympathischen Nervensystems. Bei der renalen Sympathikusdenervation (RSD) wird mit Hilfe von Hochfrequenzenergie, fokussiertem Ultraschall oder Alkoholinjektion das perivaskuläre renale Sympathikusnerven-Geflecht zerstört [588]. Bei der extrarenalen Baroreflex-Aktivierungstherapie (BAT) werden afferente Nerven des Parasympathicus im Bereich der Carotisbifurkation elektrisch stimuliert und so der Baroreflex aktiviert [589].

\section{Renale Sympathikusdenervation (RSD)}

Die perkutane RSD hat als interventionelles Verfahren mit verschiedensten Kathetersystemen sehr vielversprechende Daten in unverblindeten kontrollierten Studien an RH Patient/innen vorweisen können [588, 590], bei zwei Sham-kontrollierten Studien jedoch keinen signifikanten Effekt gezeigt [591, 592]. Im Rahmen der verblindeten, Sham-kontrollierten Simplicity-HTN3-Studie mit 535 Patient/innen wurde der primäre Endpunkt verfehlt. Die Office-BD-Senkung lag 6 Monate nach RSD mit -14,1/-6,6 mm Hg nicht signifikant unter der in der Sham-Gruppe mit $-11,7 /-4,6 \mathrm{mmHg}$ [591]. Wenngleich die mangeln- de interventionelle Erfahrung zahlreicher beteiligter Zentren mit technisch unvollständigen Ablationsprozeduren als Fehlerquelle aufgezeigt werden konnte (nur in $25 \%$ zirkumferentielle Ablation in zumindest einer Nierenarterie [593]), führten die Daten aus der Simplicity-HTN3-Studie zu einer sehr kritischen Bewertung der RSD als Therapie-Ansatz per se. Eine Meta-Analyse mit Auswertung extrahierter Daten aus Simplicity HTN-2, HTN-3, Simplicity-FLEX, HTN-JAPAN, OSLO-RDN, PRAGUE-15 und DENERHTN zeigte nur einen nicht-signifikanten Vorteil der BD-Senkung von RSD $(n=588)$ gegenüber intensivierter medikamentöser Therapie $(n=397)$, insbesondere bei Addition eines Aldosteron-Antagonisten [594]. Andererseits sollte angemerkt werden, dass nahezu alle der untersuchten Studien denselben unipolaren Ablationskatheter mit eher mäßigen Positionierungsmöglichkeiten verwendeten. Studien mit einem quadripolaren Ablationskatheter, mit dem eine zirkumferentielle Ablation der Nierenarterien in einem Ablationsschritt möglich ist, zeigten bislang günstigere Ergebnisse. In der Simplicity Spyral HTN-OFF-MED-Studie wurden Patient/innen ohne antihypertensive Medikation und Office-BD-Werten systolisch 150-180 mm Hg und diastolisch $>90 \mathrm{~mm} \mathrm{Hg}$ eingeschlossen und 1:1 versus Sham-Prozedur randomisiert [595]. Bereits nach 3 Monaten zeigte sich eine signifikant bessere Senkung des Office-BD in der Ablationsgruppe im Vergleich zur Sham-Gruppe (systolisch $-10,0 \mathrm{~mm} \mathrm{Hg}$ vs. $-2,3 \mathrm{~mm} \mathrm{Hg}, p=0,02$; diastolisch $-5,3 \mathrm{~mm} \mathrm{Hg}$ vs. $-0,3 \mathrm{~mm} \mathrm{Hg}, p=0,008$; [595]). Auch die 24-Std.-BD-Senkung war signifikant besser in der Ablationsgruppe (systolisch $-5,5 \mathrm{~mm} \mathrm{Hg}$ vs. $-0,5 \mathrm{~mm} \mathrm{Hg}, p=0,04$; diastolisch $-4,8 \mathrm{~mm} \mathrm{Hg}$ vs. $-0,4 \mathrm{~mm} \mathrm{Hg}, p<0,002$; [595]). In den Nachkontrollen traten in keiner der beiden Gruppen Nierenversagen oder Kreatinin-Anstiege über $50 \%$ des Ausgangswertes ein. In der Simplicity Spyral HTN-ON-MED-Studie wurden Patient/innen unter antihypertensiver Therapie mit 1-3 Antihypertensiva und Office-BD systolisch 150-180 mm Hg, diastolisch >90 $\mathrm{mm} \mathrm{Hg}$, untersucht [596]. Wie in der OFF-MED-Studie erfolgte eine Randomisierung versus Sham-Prozedur. Nach 6 Monaten zeigte sich auch hier eine signifikant bessere Senkung in der RSD-Gruppe im Office-BD $(-9,4 \mathrm{~mm} \mathrm{Hg}$ vs. $-2,6 \mathrm{~mm} \mathrm{Hg}$ systolisch, $p=0.0205 ;-5,2 \mathrm{~mm} \mathrm{Hg}$ vs. $-1,7 \mathrm{~mm} \mathrm{Hg}$ diastolisch, $p=0,048, n=38 / n=40)$ und im 24-Std.-BD (-9,0 mm Hg vs. $-1,6 \mathrm{~mm} \mathrm{Hg}$ systolisch, $p=0,051 ;-6,0 \mathrm{~mm} \mathrm{Hg}$ vs. $-1,9 \mathrm{~mm} \mathrm{Hg}$ diastolisch, $p=0,03, n=36 / n=36$ ). Welche Patient/innen von der RSD am meisten profitieren, ist der Gegenstand aktueller [597] und zukünftiger Forschung.

Neben der RSD mit Hochfrequenzenergie liegen mittlerweile auch für andere physikalische Verfahren Daten aus randomisierten und z.T. Sham-kontrollierten Studien vor. So wurde in der Radiance-HTNSOLO-Studie die RSD mit einer kathetergestützten nicht-fokussierten radiären Ultraschallquelle durchgeführt [598]. Durch die gleichzeitige ultraschall-be- 
dingte radiäre Wärmeemission und eine Wasserkühlung über einen zirkulären Ballon um die Ultraschallquelle herum entsteht ein ringförmiges thermisches Profil, durch das eine Ablation der Adventitia möglich ist, während Intima und Media vor Überwärmung geschützt werden [599]. Die Radiance-HTN-SOLOStudie war einfach verblindet und Sham-kontrolliert, wobei ähnlich der Simplicity Spyral HTN-OFF-MEDStudie Hypertoniker/innen mit moderat erhöhtem BD eingeschlossen wurden, bei denen in der initialen Run-in-Phase die antihypertensive Medikation vollständig abgesetzt werden konnte. Einschlusskriterium neben anatomischer Durchführbarkeit der Ablation war ein 24-Std.-BD von $\geq 135 / 85 \mathrm{~mm} \mathrm{Hg}$ und $<170 / 105 \mathrm{~mm} \mathrm{Hg}$. Der primäre Endpunkt für efficacy wurde definiert als die Änderung des Tagesintervalls im 24-Std.-BD 6 Monate nach Intervention/Sham-Prozedur. In beiden Gruppen bestand je nach Blutdruckverlauf im Follow-up die Option einer standardisierten schrittweisen Wiedereinführung der medikamentösen Therapie (SSHT) [598]. Nach 6 Monaten war bei $65 \%$ der Patienten in der RSD Gruppe eine schrittweise Einführung der antihypertensiven Medikation notwendig geworden, dagegen bei $85 \%$ in der Sham Gruppe. Obwohl bei den Patient/innen der Ablationsgruppe eine weniger intensive antihypertensive Medikation notwendig war, betrug hier die durchschnittliche BD-Senkung im 24-Std.-BD $-18,1 \pm 12,2 \mathrm{~mm} \mathrm{Hg}$ gegenüber $-15,6 \pm 13,2 \mathrm{~mm} \mathrm{Hg} \mathrm{im}$ der Sham-Gruppe. Der Unterschied zwischen RSD und Sham-Gruppe betrug nach Adjustierung für Anzahl der eingenommenen Medikamente $-4,3 \mathrm{~mm} \mathrm{Hg}$ (95\% Konfidenzintervall $-7,9$ bis $-0,6 ; p=0,024$ ) [598]. Das Novum dieser Studiendaten abgesehen vom radiären Ultraschall als Energiequelle ist die Kombination der Intervention mit standardisiertem Aufbau der medikamentösen antihypertensiven Therapie.

\section{Baroreflex Aktivierungstherapie (BAT)}

Bei der BAT wird in typischer Position pectoral ein Impulsgenerator implantiert. Zugleich wird mit lateralem Zugang in etwa auf Höhe HWK C4 die KarotisBifurkation freipräpariert und unter dem Platysma hindurch eine Stimulationslektrode vom Impulsgenerator aus zur Karotisbifurkation verlegt. Mit Hilfe eines introperativen Mapping-Verfahrens wird dann die Elektrode so auf der Karotisgabel fixiert, dass durch unipolare Simulation von der Elektrodenspitze aus der Baroreflex optimal getriggert werden kann. Für die erste Generation wurde bei Patient/innen aus 2 open-label-Studien, sowie einer randomisierten, Sham-kontrollierten Studie eine anhaltende BDSenkung bis 6 Jahre nach Device-Aktivierung gezeigt [589]. Die zweite Generation der Stimulatoren ist deutlich kleiner und benötigt nur eine unilaterale Stimulationselektrode, sodass ein besseres Sicherheits- und Verträglichkeitsprofil gezeigt werden konnte. Hierzu liegen derzeit noch keine Daten aus größeren randomisiert-kontrollierten Studien vor.

\section{Kritische Bewertung}

Nach wie vor bleibt als Manko der RSD das Fehlen zuverlässiger Prädiktoren für den Therapie-Erfolg nach dem Eingriff, sowie das Fehlen eines intraprozeduralen Endpunkts für den Ablationserfolg. Die gegenwärtigen Daten aus kontrollierten Studien sind uneinheitlich, obwohl angemerkt werden muss, dass mit den Daten aus Simplicity Spyral HTN-OFF-MED und ON-MED solide Studienergebnisse unter Shamkontrollierten Bedingungen vorliegen, die den negativen Daten aus Simplicity HTN-3 widersprechen. Die BAT wiederum stellt derzeit aufgrund des eher anspruchsvollen chirurgischen Verfahrens, der Notwendigkeit eines Aggregatwechsels bei Batterie-Erschöpfung nach 3-5 Jahren, sowie aller mit DeviceImplantation verbundenen möglichen Komplikationen eher ein Reserve-Verfahren bei Therapie-Versagen der RSD dar. Aufgrund dieser Einschränkungen wurden die interventionellen ebenso wie die Device-basierten Verfahren für die Behandlung von $\mathrm{RH}$ Patient/innen in den aktuellen ESC-Leitlinien nicht empfohlen, solange nicht verlässlichere Daten aus randomisiert-kontrollierten Studien vorliegen [52]. Auch die ACC/AHA Leitlinien aus 2017 verweisen auf die widersprüchlichen Daten aus kontrollierten Studien [116]. Beide Leitlinien sind aber vor Publikation der jüngsten, qualitativ hochwertigsten und positiven Studien erschienen.

Insgesamt erscheint es gerechtfertigt, die interventionellen Methoden der Blutdrucksenkung, insbesondere die RSD, derzeit für die klinische Routine in der Behandlung von Hypertoniepatienten nicht $\mathrm{zu}$ empfehlen. Bei ausgewählten Patienten mit RH und gesicherter Medikamenten-Compliance (Screening in Blut oder Harn) und nach Abklärung an einem Spezial-Hypertoniezentrum kann die Anwendung eines interventionellen Verfahrens bei Scheitern der medikamentösen Therapie als Option gerechtfertigt sein, insbesondere im Hinblick auf das gute Sicherheitsprofil der RSD. Die Behandlung sollte idealerweise im Rahmen kontrollierter Studien oder klinischer Register stattfinden.

\section{Hypertonie und Adhärenz}

Mangelnde Adhärenz wird zunehmend als limitierender Faktor in der erfolgreichen Behandlung von BDPatient/innen angesehen. Verglichen mit der großen Anzahl an randomisierten Multizenter-Studien zu den einzelnen antihypertensiven Substanzen, ist die Zahl der Studien zum Thema Adhärenz klein. Damit sind evidenzbasierte Aussagen nur bedingt möglich. Es zeigte sich in diesen Studien, dass die mangelnde Adhärenz einen großen Anteil am Nicht-Erreichen der BD-Ziele unserer Patient/innen hat. 
So befolgen bereits 6 Monate nach Therapiebeginn nur mehr 2/3 der Patient/innen und nach einem Jahr nur mehr $50 \%$ der Patient/innen die vereinbarte Therapiestrategie [600].

Gleichzeitig mit der Abklärung auf eine möglicherweise eingeschränkte Adhärenz sollte initial die durch die Ärztin/den Arzt bedingte therapeutische Inertia, welche am häufigsten durch mangelndes Ausdosieren und Verharren auf einer Monotherapie ein Verfehlen des Zielblutdrucks mitverursacht, ausgeschlossen werden [601].

Studien, welche die Adhärenz durch Messungen der Metaboliten von antihypertensiven Substanzen im Harn untersuchten, konnten eine niedrige Adhärenz (unter 50\%) zur Medikation, welche verordnet wurde, feststellen [602]. Mangelnde Adhärenz geht zudem mit einem erhöhten kardiovaskulären Risiko einher [603]. Vice versa zeigte sich selbst an einem betagten Patient/innenkollektiv mit einem Durchschnittsalter von 90 Jahren, dass eine Verbesserung der Adhärenz kardiovaskuläre Ereignisse und Mortalität senken konnte [519].

Die mangelnde Adhärenz ist bei bis zu $50 \%$ der Patient/innen die Hauptursache für Therapieresistenz und korreliert dabei direkt mit der Zunahme der Tablettenanzahl pro Tag [604].

Als einer der wesentlichen Gründe für fehlende Adhärenz bei Männern ist die durch Antihypertensiva ausgelöste Nebenwirkung der erektilen Dysfunktion anzuführen. Diese sollte aus diesem Grund auch bei jeder Therapieeinstellung abgefragt werden, da meist alternative Präparate zur Verfügung stehen [605].

Eine höhere Komplexität der Medikation (mehrere unterschiedliche Substanzen als Einzeltherapeutika über den Tag verteilt) führt zu verminderter Adhärenz und kann durch eine Reduktion der Gesamtzahl der täglichen Tabletten positiv beeinflusst werden. Im Speziellen kann mit einer Single-Pill-Kombination nur eine geringfügige Non-Adhärenz von $<10 \%$ registriert werden $(<20 \%$ mit zwei, $<40 \%$ mit drei Tabletten und noch höhere Non-Adhärenz mit fünf oder mehr Tabletten pro Tag) [604]. Eine bereits bei Therapiebeginn durchgeführte Kombination-Strategie aus zwei Substanzen ist außerdem mit besserer Langzeit-Adhärenz vergesellschaftet [231].

In den ESC Richtlinien zum Management nach Myokardinfarkt wurde die Polypill (Thrombozytenaggregationshemmer, Antihypertensivum, Statin) bereits als Empfehlung aufgenommen, um die Langzeit Adhärenz zu verbessern (Klasse IIA, Level B) [606].

Die Empfehlungen zur Verbesserung der Adhärenz unter antihypertensiver Therapie beinhalten ein ausführliches Gespräch mit dem Patient/innen, wie die Durchführung der medikamentösen Therapie für den Betroffenen am unkompliziertesten durchgeführt werden kann und beinhaltet das Zusammenarbeiten der den Patient/innen betreuenden Einrichtungen, Abklärung über finanzielle und örtliche Barrieren (Rezeptkosten, Entfernung zur Ärztin/zum Arzt oder zur Apotheke) und Ermutigung zum „shared decision making“, welche auch eine korrekte BD-Selbstmessung zu Hause beinhaltet. Es gibt hierbei die Evidenz, dass die BD-Selbstmessung durch den Patient/innen zu Hause die Adhärenz zur Medikation begünstigt [35, 38]. Zusätzlich konnte gezeigt werden, dass die telemetrische Rückmeldung (SMS, Nachrichtendienste) von Selbstmessungen zur Ärztin/zum Arzt die Adhärenz deutlich verbessert [607].

Eine antihypertensive Single-Pill-Kombination im Vergleich zu drei Einzelsubstanzen stellt auch eine finanzielle Erleichterung dar.

Es sollte zudem auch die somatische Seite des Patienten/der Patientin beachtet werden. Aufgrund visueller Einschränkungen, eingeschränkter Mobilität und fehlendem Bildungsgrad kann es zu einer Verschlechterung der Adhärenz kommen. Frühe Stadien einer Demenz, sowie eine manifeste Depression führen ebenfalls zu einer deutlichen Verschlechterung der Fähigkeit einer kontinuierlichen Medikamenteneinnahme. Es konnte jedoch kein Zusammenhang mit spezifischen Persönlichkeitsfaktoren gesehen werden, womit keine non-adhärente Persönlichkeit beschrieben werden konnte.

$\mathrm{Zu}$ differenzieren vom Begriff Adhärenz ist die Compliance, welcher primär eine Befolgung und Einhaltung der von Ärztin/Arzt vorgegebenen Medikation durch die Patient/innen beschreibt, wobei die Autorität und Entscheidungshoheit vollkommen der Ärztin/dem Arzt obliegt. Sollte eine Patientin oder ein Patient die vollständige Führung durch die Ärztin oder den Arzt wünschen, ist dies offen anzusprechen und zu vereinbaren.

Nur Patient/innen, die ihre Erkrankung verstehen, die Folgen kennen und diese entsprechend einschätzen können, werden auch akzeptieren, dass eine Umstellung des Lebensstils und/oder eine adäquate und dauerhafte medikamentöse Therapie die Langzeitfolgen verhindern kann. Ein regelmäßiges Abfragen über Verständnis und mögliche Nebenwirkungen gehört zur Adhärenzbildung unumgänglich dazu. Eine zeitintensivere Ersteinstellung kann somit langfristig die Adhärenzraten garantieren, die wir zur erfolgreichen Behandlung eines Bluthochdrucks unumgänglich benötigen.

Zusammengefasst beinhaltet Adhärenz eine aktive Zusammenarbeit von Ärztin/Arzt und Patientin/ Patient im Sinne einem gemeinsamen Entscheidungsfinden und Therapiezielvereinbarung.

\section{Fact Box Adhärenz}

- Nur 50 \% Adhärenz zur Blutdruck-Therapie nach einem Jahr

- Therapeutische Inertia ausschließen

- Mangelnde Adhärenz ist eine Hauptursache der therapieresistenten Hypertonie

- Single-Pill-Kombinationen führen zu verbesserter Adhärenz 
- Shared decision making (Patientin/Patient und Ärztin/Arzt) sollte forciert werden

\section{Gesprächsführung}

In der Behandlung von Patientinnen und Patienten mit arterieller Hypertonie sollte man sich bewusst sein, dass Patient/innen, die oft bis zu diesem Zeitpunkt keine körperlichen Symptome hatten und sich als gesund einschätzten, mit der Erstdiagnose einer lebenslang zumeist medikamentös zu behandelnden Erkrankung konfrontiert werden. Auf Grund des häufigen Fehlens einer klinischen Symptomatik wird die Sinnhaftigkeit der Einnahme eines Medikamentes bei möglichen Nebenwirkungen von den Patient/innen zunächst nur schwer akzeptiert.

Der Gesprächsbeginn sollte eine Information über die Dauer, die Ursachen und die möglichen Folgen des Bluthochdrucks beinhalten. Eine Abklärung auf sekundäre Hypertonie und das Abfragen möglicher vererblicher Faktoren hilft dem Patient/innen, seine Erkrankung einzuordnen und dient außerdem zur Entlastung, nicht unbedingt selbst für die Erkrankung verantwortlich zu sein. Ein Hinweis darauf, wie häufig Bluthochdruck vorkommt (nahezu die Hälfte aller Europäer jenseits des 50. Lebenjahres sind betroffen), entstigmatisiert.

Da Patient/innen häufig eine Medikamenteneinnahme mit Krankheit und Alter verbinden, sollte bei Grad 1 Hypertonie initial auf Lebensstilanpassungen zur Optimierung des BDs Wert gelegt werden, bevor eine medikamentöse Behandlung eingeleitet wird. Ein Abfragen aktueller psychischer Belastungsfaktoren bzw. Veränderungen in der Lebensweise sind zudem notwendig, um mögliche Ko-Faktoren einzubinden bzw. auszuschließen. Berufliche oder private Stresssituationen können über gesteigerte Sympathikusaktivität einen latenten Hypertonus manifest werden lassen.

Vor allem anfänglich sollte regelmäßig das vom Patient/innen Verstandene abgefragt werden und die wichtigsten Punkte, sowie auch Möglichkeiten für Mißverständnisse, wiederholt dargestellt werden.

Vor allem bei Erstdiagnose und bei Therapiebeginn ist eine kurzfristige Kontrolle (auch telefonisch oder mittels moderner Medien, wie SMS oder Nachrichtendienst möglich) zu empfehlen, um den Patient/innen die Sicherheit mit seiner Erkrankung als auch seiner Behandlung zu geben.

Die Gesprächsführung sollte motivierend sein und deeskalierend hinsichtlich nur temporär gering bzw. tolerabel erhöhten Blutdruckwerten sein.

Die 24-Stunden-Blutdruckmessung führt auf Grund des häufig geringeren Durchschnittswertes im Vergleich zu einzelnen BD-Spitzen in der Selbstmessung zu einer Deeskalation und kann gemeinsam analysiert werden.

$\mathrm{Ob}$ ein Patient oder eine Patientin eher autonom bleiben möchte und nur bei Fragen sich an ihre/n Ärz-
Tab. 42 Hypertonie-Beratung: Gesprächspunkte mit betroffenen Patientinnen/Patienten. (Adaptiert nach Prischl et al. [608])

\begin{tabular}{|c|c|c|}
\hline Gesprächsinhalte & $\begin{array}{l}\text { Erstmals ange- } \\
\text { sprochen Datum/ } \\
\text { Häkchen }\end{array}$ & $\begin{array}{l}\text { Ausreichend bespro- } \\
\text { chen Datum/Häkchen }\end{array}$ \\
\hline Diagnose Hypertonie & $\ldots$ & $\ldots$ \\
\hline $\begin{array}{l}\text { Risiken durch Hyper- } \\
\text { tonie }\end{array}$ & $\ldots$ & $\ldots$ \\
\hline $\begin{array}{l}\text { Bereitschaft zur Be- } \\
\text { handlung }\end{array}$ & $\cdots$ & $\cdots$ \\
\hline $\begin{array}{l}\text { Gewichtsreduktion/ } \\
\text {-normalisierung }\end{array}$ & $\cdots$ & $\ldots$ \\
\hline Ernährung & $\ldots$ & $\ldots$ \\
\hline $\begin{array}{l}\text { Professionelle Ernährungs- } \\
\text { beratung }\end{array}$ & $\ldots$ & $\ldots$ \\
\hline Bewegung & $\ldots$ & $\ldots$ \\
\hline Rauchen beenden & $\ldots$ & $\ldots$ \\
\hline $\begin{array}{l}\text { Alkoholkonsum einschrän- } \\
\text { ken }\end{array}$ & $\ldots$ & $\ldots$ \\
\hline $\begin{array}{l}\text { Umgang mit Stress/ } \\
\text { Belastungen }\end{array}$ & $\cdots$ & $\ldots$ \\
\hline Beruflich & $\ldots$ & $\ldots$ \\
\hline Privat & $\ldots$ & $\ldots$ \\
\hline $\begin{array}{l}\text { Professionelle Stressbera- } \\
\text { tung }\end{array}$ & $\cdots$ & $\cdots$ \\
\hline $\begin{array}{l}\text { Erste Ziele setzen/ } \\
\text { Vereinbarung treffen }\end{array}$ & $\cdots$ & $\ldots$ \\
\hline $\begin{array}{l}\text { Beginn einer Thera- } \\
\text { pie mit einem Medi- } \\
\text { kament }\end{array}$ & $\cdots$ & $\cdots$ \\
\hline $\begin{array}{l}\text { Selbstkontrolle: rich- } \\
\text { tige Blutdruckmes- } \\
\text { sung }\end{array}$ & $\cdots$ & $\cdots$ \\
\hline $\begin{array}{l}\text { Ärztliche Kontrollen: } \\
\text { wie oft, zu welchem } \\
\text { Zweck }\end{array}$ & $\cdots$ & $\cdots$ \\
\hline $\begin{array}{l}\text { Weitere Medika- } \\
\text { mente, zu welchem } \\
\text { Zweck }\end{array}$ & $\cdots$ & $\cdots$ \\
\hline $\begin{array}{l}\text { Weitere Kontrollen, } \\
\text { zu welchem Zweck }\end{array}$ & $\cdots$ & $\ldots$ \\
\hline $\begin{array}{l}\text { Weitere Ziele/ } \\
\text { Vereinbarungen tref- } \\
\text { fen }\end{array}$ & $\ldots$ & $\ldots$ \\
\hline $\begin{array}{l}\text { Mess- und Kontroll- } \\
\text { befunde besprechen }\end{array}$ & $\ldots$ & $\ldots$ \\
\hline
\end{tabular}

tin/Arzt wendet, oder eine vollständige Führung mit regelmäßiger Kontrolle erfolgt, sollte offen angesprochen und entsprechende Termin oder Kontaktaufnahme angepasst werden.

Tab. 42 zeigt einen Vorschlag, welche Punkte erörtert werden sollten, wenn man eine Patientin/einen Patienten mit arterieller Hypertonie zu behandeln beginnt.

Die Liste ist als Vorschlag und Hilfe zu verstehen, sie erhebt keinen Anspruch auf Vollständigkeit. Auch die Reihung der Punkte folgt keiner bestimmten Logik und ist individuell anzupassen. Weitere Punkte aus 
der eigenen Erfahrung der Ärztin/des Arztes können/ sollen ergänzt werden.

\section{Welche Medikamente sollten vermieden wer- den?}

Eine beträchtliche Anzahl von Substanzen kann die Wirkung von antihypertensiven Medikamenten beeinträchtigen bzw. den BD erhöhen. Daher ist eine ausführliche Medikamentenanamnese, sowie die Anamnese hinsichtlich alternativmedizinischer Präparate, Tees, Drogen und Energy Drinks unumgänglich.

\section{Nichtsteroidale Antirheumatika (NSAR)}

NSAR zählen weltweit zu den am häufigsten verschriebenen Substanzen und finden sich daher auch häufig als Co-Medikation bei Patient/innen mit Hypertonie. Als Substanzklasse bewirken NSAR eine BD-Steigerung des arteriellen Mitteldrucks um etwa $5 \mathrm{~mm} \mathrm{Hg}$ [609], wobei für Patient/innen mit vorbestehender Hypertonie die größte Wahrscheinlichkeit einer Blutdrucksteigerung besteht [610]. NSARs bewirken eine herabgesetzte Synthese von Prostaglandin E2 und Prostazyklin, welche eine Verringerung des renalen Blutflusses, gesteigerte Salz- und Wasserretention, sowie eine Vasopressin-vermittelte Steigerung des peripheren vaskulären Widerstands zur Folge haben [76, 611]. Die verschiedenen Klassen der NSAR unterscheiden sich jedoch beträchtlich in ihrem Wirkprofil.

In einer doppelblind randomisierten Studie bei Patient/innen mit rheumatoider Arthritis oder Osteoarthritis mit kardiovaskulären Erkrankungen oder hohem kardiovaskulärem Risiko (PRECISION-ABPM) wurde der BD-Effekt der Einnahme von Ibuprofen (600-800 mg 3× tgl) mit Celecoxib (100-200 mg $2 \times$ tgl) und Naproxen (375-500 mg $2 \times$ tgl) verglichen [612]. Nach 4 Monaten lag die Zunahme des systolischen BDs im 24-h-BD-Monitoring jeweils bei $3,7 \mathrm{~mm} \mathrm{Hg}$ (95\% CI, 1,72; 5,58 mm Hg), $-0,3 \mathrm{~mm} \mathrm{Hg}(95 \% \mathrm{CI}$, 2,25; $1,74 \mathrm{~mm} \mathrm{Hg})$ und $1,6 \mathrm{~mm} \mathrm{Hg}(95 \% \mathrm{CI},-0,40$; $3,57 \mathrm{~mm} \mathrm{Hg}$ ). Der Anteil der initial normotensiven Patient/innen, die im Laufe der Behandlung eine Hypertonie entwickelte (24-h-BD syst. $>130 \mathrm{~mm} \mathrm{Hg}$ ) betrug 23,2\% für Ibuprofen, 10,3\% für Celecoxib und $19,0 \%$ für Naproxen [612]. Andererseits besteht für alle NSARs eine deutliche Dosisabhängigkeit der Wirkung auf den BD. Die in der PRECISION-ABPM-Studie verwendete Dosis von Celecoxib war eher moderat. Für die Dosis $400 \mathrm{mg} 2 \times$ tgl. konnte dagegen in einer Studie an Patient/innen mit kolorektalen Adenomen eine signifikante Zunahme des systolischen OfficeBDs um 2,9 mm Hg nach $1 \mathrm{Jahr}$ und 5,2 mm Hg nach 3 Jahren gezeigt werden [613].

In einer Meta-Analyse aus 2009 wurde auf die Heterogenität der bisherigen Studiendaten zum BD-Effekt von NSAR hingewiesen, dennoch war hier die BDSteigerung in der Klasse der selektiven COX-2-Inhibitoren größer als bei den nicht-selektiven Substanzen.
Die größten BD-Steigerungen lagen vor bei Rofecoxib und Etoricoxib, während Celecoxib, Valdecoxib und Lumaricoxib kaum Einfluss auf den BD hatten [614]. In einem anderen Review traten die größten BD-Steigerungen bei Einnahme von Indomethacin, Naproxen und Piroxicam auf [610].

NSAR bewirken zudem eine Verringerung des antihypertensiven Effekts von Diuretika, Betablockern, ACE-Hemmern und ARBs. Daher sollten bei NSARinduzierter Hypertonie bzw. Verschlechterung der BD Kontrolle bei Hypertonikern unter Einnahme von NSAR Kalzium-Antagonisten und zentrale Sympatholytika bevorzugt werden $[615,616]$.

Umgekehrt wird bei Patient/innen mit arterieller Hypertonie Paracetamol als Analgetikum der Wahl zur Therapie von rheumatologisch oder entzündlich bedingten Schmerzen empfohlen, da Paracetamol nur einen geringen Einfluss auf den BD hätte [76]. Aufgrund widersprüchlicher Studien kommt jedoch auch eine aktuellere Meta-Analyse aus 2013 zu dem Fazit, dass der Effekt von Paracetamol auf die BD-Einstellung bei hypertensiven Patienten nicht klar charakterisiert werden kann. Während Observationsstudien mit einer Gesamtheit von 147.000 Patienten eine BDSteigerung zeigten, kamen prospektiv randomisierte, überwiegend kleine Studien zu keinem eindeutigen Trend [617].

\section{Antidepressiva}

Partient/innen mit Depression weisen typischerweise niedrige $\mathrm{BD}$-Werte auf, wobei das sympathische Nervensystem unterschiedliche Aktivitäten aufweisen und je nach psychischen Störungsbildern sowohl vermindert als auch vermehrt aktiviert sein kann [618]. Dennoch können verschiedene antidepressive Substanzen dosisabhängig zu einer BD-Erhöhung führen. Dies wurde im Rahmen großer Meta-Analysen sowohl für trizyklische Antidepressiva (TCA) [619], als auch für systemische Serotonin Wiederaufnahme-Hemmer (SSRI) gezeigt [620], gilt aber auch für andere Klassen von Antidepressiva. In einer niederländischen Studie an 2981 Patient/innen, davon 2018 mit Depression oder Angststörung, war sowohl der systolische, als auch der diastolische BD signifikant höher in der Gruppe mit Behandlung mit TCA [619]. Venlafaxine als Vertreter der SSRI führte dagegen dosisabhängig überwiegend $\mathrm{zu}$ einer Steigerung des diastolischen BDs, wobei das Risiko für diesen Effekt besonders groß bei männlichen Patienten und älteren Personen war [620]. Patient/innen unter SSRI-Therapie zeigen eine verringerte Baroreflex-Sensitivität und Herzfrequenz-Variabilität als Zeichen einer reduzierten Vagus-Aktitivät [621]. Zudem bewirken SSRI ebenso wie TCA unmittelbar am Herzen eine Hemmung der Wiederaufnahme von Noradrenalin in sympathische Nervenenden und erzeugen so eine gesteigerte sympathische Aktivierbarkeit mit Erhöhung des kardialen Output und resultierender Hypertonie [622]. 
Bei der Behandlung von Hypertonie unter antidepressiver Therapie sollte beachtet werden, dass einige antihypertensive Substanzen selbst wiederum mit Gemütsstörungen assoziiert sein können. In einer grossen single-center Studie an 144.066 Patient/innen mit 5 Jahren follow-up hatten Patient/innen unter Therapie mit Betablockern oder Kalziumantagonisten ein doppelt so hohes Risiko für eine stationäre Aufnahme wegen Depressionen oder bipolaren Störungen wie unter Therapie mit ACE-Hemmern oder ARBs, welche das niedrigste Risiko zeigten [623].

\section{Hormonelle Kontrazeption und Hormonersatztherapie (HRT)}

Bei Patientinnen mit einer arteriellen Hypertonie oder schwangerschafts-induzierter Hypertonie in der Vorgeschichte, sowie bei Frauen afro-amerikanischer Abstammung besteht ein höheres Risiko für die Entwicklung einer Hypertonie während oraler Kontrazeption (OK), pathophysiologisch vor allem aufgrund RAASvermittelter vermehrter Salz- und Wasser-Retention. Dieser Effekt ist stark abhängig von der Substanzklasse der OK und betrifft insbesondere ÖstrogenMonopräparate oder ältere Kombinationspräparate mit hoher Östrogen-Dosis $(>30 \mu \mathrm{g})$. Bei etwa $5 \%$ der Frauen, die eine hormonelle OK mit mindestens $50 \mu \mathrm{g}$ Östrogen und 1-4 mg Progestagen einnehmen, entwickelt sich eine Hypertonie [502]. Für monophasische Kombinationspräparate mit $30 \mu \mathrm{g}$ Östrogen und gleichbleibender Progestagen-Dosis während des gesamten Menstruations-Zyklus ist ebenfalls eine $\mathrm{Zu}$ nahme des 24-h-BDs um $8 \mathrm{~mm} \mathrm{Hg}$ systolisch gezeigt worden [624]. Bei moderneren bi- oder triphasischen Kombinationspräparaten, bei denen die Dosis des Progestagens entsprechend dem natürlichen Zyklusverlauf variiert, ist das Risiko für die Entwicklung einer Hypertonie während der OK geringer [502]. Für OK-Präparate der 4. Generation mit Drospirenon als Progestagen, welches eine anti-mineralokortikoide Wirkung auf das RAAS besitzt, wurde ein neutraler bis gering BD-senkender Effekt gezeigt, was insbesondere bei Patientinnen mit hohem kardiovaskulärem Risiko wertvoll erscheint [625]. Für eine Monotherapie mit Progestagen dagegen konnte innerhalb von 2-4 Jahren follow-up keine Assoziation mit der Entstehung von arterieller Hypertonie gezeigt werden [626]. Auch Verhütungsringe mit kombinierter Hormontherapie, die $15 \mathrm{mcg}$ Ethinylestradiol und 120mcg Etonogestrel täglich freisetzen, können v.a. zu diastolischer Hypertonie führen [627].

Somit sind bei Patientinnen mit bekanntem Hypertonie-Risiko neuere triphasische Kombinationspräparate der dritten oder vierten Generation mit geringer Östrogendosis zu empfehlen. Solange eine suffiziente Kontrazeption durchgeführt wird, besteht keine Kontraindikation gegen die Verwendung effizienter Antihypertensiva wie ACE-Hemmer oder ARBs. Sobald ein Kinderwunsch besteht, müssen mit der ora- len Kontrazeption auch diese Substanzklassen abgesetzt und der BD neu evaluiert werden. Falls erforderlich ist dann eine Schwangerschafts-kompatible antihypertensive Therapie einzuleiten (s. Abschn. 18).

Die überproportionale Zunahme von Patientinnen mit arterieller Hypertonie im Verlauf der Menopause und dem postmenopausalen Lebensabschnitt legt eine besondere pathophysiologische Bedeutung des Wegfalls von Östrogen nahe. Dennoch liegen bereits für den Nachweis dieser Kausalität zwischen Menopause und Hypertonie widersprüchliche Studiendaten vor [628]. Dasselbe gilt für den Effekt von HRT auf die Häufigkeit bzw. die Therapiekontrolle von arterieller Hypertonie. Der erhoffte Effekt einer Reduktion der Häufigkeit von Hypertonie durch HRT konnte bislang nicht konsistent gezeigt werden, umgekehrt besteht kein klarer Nachweis eines Risikos von Hypertonie durch postmenopausale HRT [628, 629].

\section{Immunsuppressiva}

Bei Organtransplantationen und Knochenmarkstransplantationen hat die Therapie mit dem CalcineurinInhibitor Cyclosporin zu einer deutlichen Verbesserung der Allograft-Prognose und der Mortalität geführt, sodass im Laufe der letzten Jahrzehnte die Verwendung auf nicht-transplantationsbedingte Indikationen wie Psoriasis, Myasthenia gravis und rheumatoide Arthritis ausgeweitet wurde. In der Gruppe der Immunsuppressiva löst Cyclosporin am häufigsten dosisabhängig einen BD-Anstieg aus. Die Häufigkeit von Cyclosporin-induzierter Hypertonie ist deutlich von der jeweiligen Indikation abhängig. Sie findet sich in 30-60\% der Patient/innen nach Knochenmark-Transplantationen, $65-85 \%$ nach Nieren- oder Lebertransplantationen, wobei vor Nierentransplantation bereits $50 \%$ der Patient/innen eine schwere Hypertonie aufweisen, und 70-100\% der Fälle nach Herztransplantation [630]. Pathophysiologisch liegt der Cyclosporin-induzierten Hypertonie eine systemische und renale Vasokonstriktion mit Verringerung des renalen Blutflusses, eine renale Natriumretention sowie eine direkte nephrotoxische Wirkung zugrunde. Typischerweise treten dabei Störungen des zirkadianen Rhythmus mit Fehlen oder Inversion des nächtlichen BD-Dipping auf [76, 630]. Tacrolimus, ein mit Cyclosporin strukturverwandter und ebenfalls nephrotoxischer Calcineurin-Inhibitor, führt geringfügig seltener zu Hypertonie nach Transplantation, wenngleich dieser Unterschied nicht immer statistisch signifikant war.

Die Wahl der Antihypertensiva bei Cyclosporininduzierter Hypertonie sollte den Wirkmechanismus von Cyclosporin berücksichtigen. Unter Cyclosporingabe ist die Aktivität des RAAS und die renale GFR reduziert, die renale Exkretion von Kalium und $\mathrm{H}^{+}$-Ionen partiell gehemmt, sodass ein erhöhtes Risiko für eine hyperkaliämische metabolische Azidose besteht. Der Harnsäure-Spiegel ist oft erhöht. Die Effektivität 
einer RAAS-Blockade mit ACE-Hemmern oder ARBs ist daher sehr gering, unter Therapie mit Diuretika besteht ein erhöhtes Risiko für zunehmende Niereninsuffizienz und Hyperurikämie. Mittel der Wahl sind daher Kalziumantagonisten vom Dihydropyridin-Typ oder Betablocker, ggf. auch beide in Kombination. Andererseits muss beachtet werden, dass alle Kalziumantagonisten Inhibitoren des Cytochrom P450 3A4 Isoenzyms sind, über welches auch Cyclosporin A, Tacrolimus und Sirolimus abgebaut werden. Im Rahmen kleinerer Observationsstudien betrug die Zunahme des Cylosporin A Spiegels unter Therapie mit Amlodipin 40\% [631], mit Lercanidipin 20\% [632] und mit Felodipin 16\% [633].

Bei schwer einstellbarer Hypertonie oder relevanter Nephrotoxozität kann ein Wechsel von Cyclosporin A oder Tacrolimus auf immunsuppressive Kombinationstherapien mit Mycophenolat Mofetil oder dem m-TOR-Inhibitor Sirolimus erwogen werden, die weniger nephrotoxisch sind, keine systemische Vasokonstriktion verursachen und für die allenfalls geringe Effekte auf den BD gezeigt worden sind [634].

\section{Antineoplastische Substanzen}

VEGF-Inhibitoren haben die Therapiemöglichkeiten bei metastasierten soliden Tumoren revolutioniert und die Prognose der Patient/innen deutlich verbessern können. Erstmanifestation oder Verschlechterung einer Hypertonie zählt zu den häufigsten Nebenwirkungen unter Therapie mit VEGF-Inhibitoren, wobei bei der Mehrzahl der Patient/innen eine Hypertonie vorbekannt war [635]. Bevacizumab ist ein monoclonaler Antikörper gegen VEGF-A und war der erste zugelassene VEGF-Inhibitor. Unter Bevacizumab Therapie treten dosisabhängig Hypertonien auf, in einer Meta-Analyse war das relative Risiko für Hypertonie im Niedrigdosis-Bereich $(3,0 ; 5,0$ oder $7,5 \mathrm{mg} /$ kgKG) 3,0 ; bei Hochdosis-Therapie (10 oder $15 \mathrm{mg} /$ kgKG) 3,5 [636]. 36-50\% der behandelten Patient/ innen zeigten einen BD-Anstieg, wobei der Anstieg eher moderat war und der systolische BD mehr betroffen war als der diastolische. Schwere Hypertonien mit BD >200/100 mm Hg traten im Vergleich zu Placebo 3- bis 5-mal häufiger unter Therapie mit Bevacizumab auf, bei $1 \%$ der Patienten lebensbedrohliche hypertensive Krisen [76, 635]. Die Zeitdauer bis zum BDAnstieg war sehr variabel und lag im Median in einer Studie bei 131 Tagen.

Tyrosin-Kinase Inhibitoren wirken durch Hemmung von VEGF-Rezeptoren oder Plättchen-abhängigen Wachstumsfaktor (PDGFR $\alpha, \operatorname{PDGRF} \beta$, FLT-3) antineoplastisch. $\mathrm{Zu}$ dieser Gruppe gehören Sorafenib, Sunitinib, Lapatinib, Axitinib und Pazopanib. In einer Studie zu Sofratenib bei 903 Patient/innen mit Nierenzell-Karzinomen trat Hypertonie in $17 \%$ der Fälle unter Therapie mit $2 \times$ tgl. $400 \mathrm{mg}$ Sorafenib auf. In $4 \%$ der Fälle traten Hypertonie-Episoden Grad 2 auf [637]. In einer Studie mit 24-Std.-BD-Messung trat unter Sorafenib $400 \mathrm{mg} 2 \times$ tgl. eine BD-Steigerung von 8,2 mm Hg systolisch und 6,2 mm Hg diastolisch während der ersten $24 \mathrm{~h}$ nach Therapiebeginn auf [638].

Sunitinib ist zugelassen für die Therapie von Nierenzell-Karzinomen und solide Tumoren des Gastrointestinaltrakts. In einer Meta-Analyse wurden 4999 Patient/innen aus 13 prospektiv randomisierten klinischen Studien untersucht. Während vor Therapiebeginn bei keinem Patienten/keiner Patientin Hypertonie vorbekannt war, trat unter Therapie mit $50 \mathrm{mg} / \mathrm{d}$ Sunitinib in $22,5 \%$ der Patient/innen $(95 \%$ CI: 19,5-25,9\%) eine arterielle Hypertonie auf. Schwere hypertensive Krisen traten bei $6,8 \%$ der Patient/ innen auf (95\% CI: 5,3-8,8\%) [639], und das relative Risiko für hypertensive Krisen unter Sunitinib-Therapie betrug 22,72 (95\% CI: 4,48-115,29; $p<0,001$ ). Zudem zeigte sich ein erhöhtes Risiko für Nierenfunktionsstörungen mit Anstieg des Serum Kreatinins (RR 1,36; $95 \%$ CI: 1,20-1,54; $p<0,001$ ) [639]. Andererseits konnte für Bevacizumab [640], Sorafenib [641], und Sunitinib [642] gezeigt werden, dass das Auftreten einer arteriellen Hypertonie ein klinischer Marker für ein günstiges Ansprechen auf die Tumor-Therapie war. Sowohl für Sorafenib als auch für Sunitinib werden wöchentliche BD Kontrolluntersuchungen während der ersten 6 Wochen unter Therapie empfohlen [639].

Im Hinblick auf den pharmakologischen Mechanismus liegt bei VEGF-Inhibitoren ein Klasseneffekt vor. VEGF-Inhibition führt $\mathrm{zu}$ verringerter Bildung von NO-Synthetase (NOS) und NO in vaskulären Endothelzellen von Arteriolen und anderen Widerstandsgefäßen, was eine Steigerung des vaskulären Tonus und eine Gefäßrarefikation zur Folge hat. Hierdurch steigt letztlich der periphere Widerstand und die antioxidative Wirkung von NO wird vermindert [76, 635]. Zugleich bewirkt die verminderte NO Produktion in renalen Endothelzellen eine Dysregulation der VEGF Expression, wodurch thrombotische Mikroangiopathien entstehen können [635].

Bei VEGF-Inhibitor assoziierter Hypertonie wurden bislang erfolgreich ACE-Hemmer, ARBs, Kalziumantagonisten, Diuretika, Betablocker und Nitrate eingesetzt. Da bei VEGF-Inhibitor Therapie auch das Risiko für Proteinurie erhöht ist, spricht für ACE-Hemmer/ ARBs deren protektiver Effekt gegenüber Proteinurie bei diabetischer Nephropathie sowie eine Verbesserung der Endothelfunktion und der renalen Mikrozirkulation [635]. Zudem wurde sowohl für ACE-Hemmer, als auch für ARBs ein antineoplastischer Effekt durch Verlangsamung von Teilung und Differenzierung von Tumorzellen gezeigt, am ehesten aufgrund der durch RAAS-Blockade stattfindenden Hemmung der Angiotensin-2-Synthese, einem potenten Mitogen und zellulären Wachstumsfaktor [643]. Kalziumantagonisten vom Dihydropyridin-Typ bewirken ebenfalls eine periphere Vasodilatation und werden von einigen Autoren als Erstlinien-Therapie bei VEGF-induzierter Hypertonie empfohlen [635]. Im Hinblick auf die pa- 
thophysiologische Eigenschaft des NO-Antagonismus von VEGF-Inhibitoren ist eine Therapie mit Nitraten, PDE-Inhibitoren oder Nebivolol naheliegend, die alle eine gesteigerte endotheliale NO-Produktion bewirken. Für langwirksame Nitrate konnte zumindest in kleinen Fallserien ein günstiger Effekt auch bei bereits bestehender antihypertensier Standard-Therapie gezeigt werden, sodass bei Ansprechen auf eine Test-Dosis von 5-10 mg Isosorbid-Dinitrat eine additive Gabe empfohlen wird [635, 644]. Auch bei Nebivolol entsteht der BD-senkende Effekt neben der selektiven $\beta 1$ Rezeptorblockade überwiegend durch eine periphere Vasodilatation durch Aktivierung des endothelialen L-arginin NO-Systems. Somit wäre auch hier ein günstiger Antagonismus zur verringerten NO-Bildung unter VEGF-Inhibition gegeben [645].

Kontraindiziert sind nicht-Dihydropyridin Kalziumantagonisten wie Verapamil und Diltiazem, die beide das hepatische Cytochrom P450 3A4 hemmen, und somit mit dem Abbau von VEGF-Inhibitoren außer Bevacizumab (anderer Abbauweg) interagieren können [635]. Der Dihydropyridin-Kalziumantagonist Nifedipin wiederum bewirkt eine VEGF-Sekretion [646], und sollte daher auch nicht zum Einsatz kommen [635].

\section{Erythropoietin}

Recombinantes humanes Erythropoietin (r-HuEPO) wird bei Niereninsuffizienz und in der Tumortherapie eingesetzt. Bei $20-30 \%$ der Patienten tritt in einem Zeitintervall von 2 Wochen bis 4 Monate nach Therapiebeginn mit r-HuEPO oder anderen Erythropoesestimulierenden Substanzen (ESA) dosisabhängig eine arterielle Hypertonie auf, bzw. verschlechtert sich eine vorbestehende Hypertonie [76]. Bei Hämodialyse-Patienten trat bei intravenöser Gabe von 40, 80 oder $120 \mathrm{U} / \mathrm{kg}$ r-HuEPO für 48 Wochen in jeweils $28 \%$, 32\% bzw. 56\% der Patienten in den Behandlungsgruppen eine arterielle Hypertonie auf. Der BDAnstieg betrug im Mittel 5-8 mm Hg systolisch und 4-6 mm Hg diastolisch [647]. Risikofaktoren für die Entstehung einer EPO/ESA-induzierten Hypertonie umfassen intravenöse Gabe, Hämodialyse (im Gegensatz zu Peritonealdialyse), familiäre Vorbelastung für arterielle Hypertonie, hohes $\mathrm{Hb}$-Therapieziel und hohe r-HuEPO/ESA Dosis [648]. Trotz zahlreicher Daten aus experimentellen und klinischen Studien ist der Mechanismus der Induktion einer Hypertonie durch Gabe von r-HuEPO/ESA noch nicht vollständig aufgeklärt. Pathophysiologisch existiert eine Dissoziation zwischen dem Effekt von r-HuEPO auf die Erythropoese und dem Effekt auf den BD. Konsistent hiermit konnte gezeigt werden, dass durch Anheben des Hämatokrits auf gleiche Werte durch Erythrozyten-Transfusion der BD eher abfiel, während er bei r-HuEPO Administration anstieg [647]. Es scheint auf dem r-HuEPO Molekül unterschiedliche Epitope zu geben, von denen das eine Erythropoese bewirkt und
Tab. 43 Blutdrucksteigernde Arzneimittel und Substanzen. (Adaptiert nach [52])

\begin{tabular}{|c|c|}
\hline Orale Kontrazeptiva & Insbesondere bei Östrogengehalt $>30 \mu \mathrm{g} / \mathrm{Tbl}$ \\
\hline Diät-Tabletten & Phenylpropanolamin, Sibutramin \\
\hline $\begin{array}{l}\text { Abschwellende } \\
\text { Nasensprays }\end{array}$ & Phenylephrin Hydrochlorid, Naphazolin Hydrochlorid \\
\hline $\begin{array}{l}\text { Stimulierende } \\
\text { Drogen }\end{array}$ & Amphetamine, Kokain, Ecstasy \\
\hline Lakritze & Glycyrrhizin \\
\hline $\begin{array}{l}\text { Immunsuppressive } \\
\text { Therapie }\end{array}$ & Cyclosporin A, Tacrolimus, Steroide \\
\hline $\begin{array}{l}\text { Antiangiogene } \\
\text { Tumortherapien }\end{array}$ & $\begin{array}{l}\text { VEGF Inhibitor (z. B. Bevacizumab), Tyrosinkinase } \\
\text { Inhibitoren (z. B. Sunitinib), Sorafenib }\end{array}$ \\
\hline Andere Substanzen & $\begin{array}{l}\text { Anabolika, Erythropoietin, NSAR, Kräutermischungen } \\
\text { (z. B.:Ephedra; Ma Huang) }\end{array}$ \\
\hline
\end{tabular}

das andere vaskuläre Effekte mit BD Anstieg vermittelt. Unabhängig vom Hämatokrit-Anstieg [649] und unabhängig vom Anstieg der Blutviskosität bewirkt r-HuEPO eine erhöhte Endothelin-1-Ausschüttung, eine Reduktion von vasodilatierendem Prostazyklin (PGI2) und eine vermehrte Bildung von vasokonstringierendem Thromboxan (TBX2). Systemisch bewirkte r-HuEPO zudem eine Verringerung der endothelialen eNOS- und NO-Produktion, sowie insbesondere bei Hämodialyse-Patient/innen eine erhöhte Sensitivität gegenüber Noradrenalin und Angiotensin-II [635]. Therapeutisch sollte präventiv darauf geachtet werden, den $\mathrm{Hb}$ langsam anzuheben und als Ziel-HbSpiegel nicht wesentlich über $10-11 \mathrm{~g} / \mathrm{dl}$ anzustreben [650]. Sollte eine antihypertensive Medikation erforderlich werden, sind ACE-Hemmer, AT1-ARBs, Kalziumantagonisten oder direkte Vasodilatatoren möglich, sofern von Seiten einer dialysepflichtigen Niereninsuffizienz keine Kontraindikationen bestehen.

\section{Coffein}

Coffein wurde über lange Zeit als blutdrucksteigernd diskutiert, hier muss jedoch zwischen akuter Wirkung und chronischem Effekt von Coffein einerseits, und zwischen der isolierten Wirkung von Coffein und dem BD-Effekt Coffein-haltiger Getränkte als Stoffgemische (z. B. Kaffee) andererseits differenziert werden [76]. Der akute kurzfristige Anstieg nach Konsum scheint gesichert, eine sichere Evidenz für eine Assoziation mit chronischer Hypertonie liegt aber nicht vor. Auch verschiedene Kräutermischungen können blutdrucksteigernde Effekte haben, die Datenlage ist hier aber vage [76].

Eine Auflistung der wichtigsten blutdrucksteigernden Substanzen bietet Tab. 43 [5].

\section{Blutdruckschulung und Empowerment}

Lifestylemodifikation wird als primäre Therapiemaßnahme in allen Hypertonie-Stadien und -Vorstufen empfohlen [52]. Die Umsetzung selbiger gestaltet sich 
für den Großteil der Patient/innen aber als schwierig, zum einen aufgrund mangelnden Wissens, zum anderen aufgrund fehlender Konsequenz und Durchhaltevermögens. Patient/innen benötigen eine ausführliche Information und Empfehlung zur Umsetzung von Lifestylemaßnahmen, wofür im ärztlichen Alltag kaum Ressourcen zur Verfügung steht.

Gut ausgebildete Apotheker/innen können durch Medikationsmanagement in Zusammenarbeit mit einem Arzt/einer Ärztin das Blutdruckmanagement verbessern [651]. Auch geschultes Krankenpflegepersonal kann durch Lifestyle- und Gesundheitsschulungen bei chronisch Kranken und Hypertoniker/innen entscheidend zur Verbesserung beitragen [652, 653]. Team-basierte Ansätze, die unterschiedliche Professionalitäten aus dem Gesundheitswesen und damit unterschiedliche Expertisen zusammenführen und dem gemeinsamen Ziel der Blutdrucksenkung dienen, sind in $\mathrm{Zu}-$ kunft unumgänglich. Auch eine Unterstützung durch Devices mit Telemonitoring zur Therapieeinleitung, Adaptierung und Kontrolle erscheint interessant.

Strukturierte multidisziplinäre Schulungsprogramme stellen eine exzellente Möglichkeit für Hochdruckpatienten dar und zeigen in einer randomisierten Untersuchung anhaltende blutdrucksenkende Effekte [36].

Eine Schulung sollte folgende Punkte in individualisierter Form beinhalten:

1. richtige Blutdruckselbstmessung und Dokumentation

2. Salz-und cholesterinarme Ernährung, die reich an Kalium/Magnesium, Obst und Gemüse ist (soferne keine Niereninsuffizienz vorliegt)

3. regelmäßige Ausdauerbewegung, ggf. auch Tonustraining

4. Basiswissen über antihypertensive Medikation, Nebenwirkungen und hypertensive Entgleisung

5. Nikotinentwöhnung, Entspannungsmethoden

Durch mehrmaliges Treffen der Patient/innen in Kleingruppen, angeleitet durch speziell geschultes diplomiertes Pflegepersonal und Ärzte/Ärztinnen, werden Allgemeinmaßnahmen in verständlicher Weise vermittelt und alltagsgerechte Vorschläge gemeinsam mit dem Patient/innen erarbeitet.

Eine wesentliche Rolle hierbei spielt auch das Empowerment, also die Patient/innenmotivation. Es hat sich gezeigt, dass gemeinsam getroffene Zielvereinbarungen am Ende einer Schulung ein praktikables Tool darstellen, um Zielwerte auch bei Kontrollen nach 1-2 Jahren einzuhalten und damit langfristig umzusetzen [36]. Der Erfolg des Programmes lässt darauf schließen, dass besser informierte Patient/ innen, denen eine Eigenverantwortung für ihre Erkrankung nähergebracht wurde, ihre vorgeschriebene Therapie regelmäßiger und genauer einnehmen und damit eine bessere Blutdruckeinstellung erlangen.

In Österreich steht derzeit ein Schulungsprogramm in dieser strukturieren Form, über mehrere Einheiten nur in der Steiermark unter dem Namen Herz.Leben und in gestraffter Form in Salzburg auf remunerierter Basis zur Verfügung. Ein flächendeckendes Schulungsprogramm für Patient/innen mit Bluthochdruck, bzw. ein Disease Management Programm Hypertonie werden langfristig angestrebt, um die Umsetzung von Lifestylemaßnahmen und Therapieadhärenz zu verbessern. In weiterer Folge wäre auch ein umfassenderes Disease Management Programm kardiovaskuläre Prävention anzudenken.

\section{Kontrollen und Kontrollintervalle}

Bei erstmals diagnostizierter arterieller Hypertonie und/oder noch nicht erreichten Therapiezielen sind v. a. anfangs häufige BD-Kontrollen erforderlich (siehe die entsprechenden Kapitel weiter vorne). Da es jedoch auch bei bereits zufriedenstellend eingestellter Hypertonie zu Anstieg, Abfall und/oder ausgeprägten Schwankungen des BDs kommen kann, sind bei allen Patient/innen regelmäßig weitere BD-Kontrollen erforderlich. Bei bereits vorliegenden normotensiven Meßwerten sind anfangs regelmäßige ärztliche Kontrollen (z.B. alle 2 Monate) erforderlich, wobei v. a. auch die Adherence des Patienten/der Patientin sowie eventuell auftretende Nebenwirkungen immer gezielt nachgefragt werden sollten. Sowohl zur Beurteilung der Langzeit-Blutdruckontrolle als auch zur Förderung der Compliance ist die BD-Selbstmessung ideal geeignet.

Die Häufigkeit von BD-Kontrollen hängt dabei auch vom Schweregrad der Hypertonie sowie vom eventuellen Vorliegen von Begleiterkrankungen ab: Bei gut kontrolliertem BD und weitgehender Beschwerdefreiheit des Patienten/der Patientin genügen Kontrollen im Abstand von 3-6 Monaten. Dabei sind die Patient/ innen jedoch auch ganz gezielt aufzuklären, neu aufgetretene Beschwerden möglichst sofort $\mathrm{zu}$ melden und damit nicht „bis zur nächsten geplanten Kontrolle $\mathrm{zu}$ warten“.

Die Häufigkeit von Ordinations-Kontrollen kann auch dadurch reduziert werden, daß die Ergebnisse von BD-Selbstmessungen elektronisch direkt zum Hausarzt/zur Hausärztin oder zur Internistin/zum Internisten übertragen werden, sodaß diese/r die Möglichkeit bekommt, aktuelle BD-Werte auch ohne Anwesenheit des Patient/innen zu kontrollieren und bei Bedarf darauf entsprechend $\mathrm{zu}$ reagieren.

Patient/innen, bei denen sich keine Hypertonie sondern „nur“ ein hoch-normaler BD (130-139/ 85-89 mm Hg bei Arztmessung) findet, zeigen häufig zusätzliche Risikofaktoren und/oder Hypertonievermittelte Organschäden (dann sollte das Vorliegen einer maskierten Hypertonie ausgeschlossen werden) sowie auch ein erhöhtes Risiko, eine manifeste Hypertonie zu entwickeln. Es empfehlen sich daher auch bei Patient/innen mit hoch-normalem BD bereits mindestens jährliche Kontrollen mit dabei immer klaren 
Empfehlungen v. a. im Hinblick auf entsprechende Lebensstiländerungen.

Dabei spielen auch umfassende Aufklärung und Motivation des Patienten/der Patientin eine ganz wesentliche Rolle, um die Bedeutung von Hypertonie möglichst klar bewusst zu machen („Hoher BD ist gefährlich obwohl man diesen gar nicht spürt!"), und daß er/sie nicht darum herum kommt, dafür auch die Verantwortung zu übernehmen und diese selbst zu tragen (siehe auch Abschn. 28 Blutdruckschulung und Empowerment): Je besser ein Patient/eine Patientin aufgeklärt ist, umso mehr steigt nicht nur die Compliance/Adhärenz sowohl im Hinblick auf regelmäßige Einnahme der antihypertensiven Medikamente (wobei niedrige Compliance auch die häufigste Ursache schlechter BD-Einstellung darstellt) als auch auf Regelmäßigkeit und Dokumentation der BD-Selbstmessungen sowie der bei den meisten Patienten/-innen erforderlichen „Lifestyle Modification“. Dadurch werden letztlich auch immer längere Kontrollintervalle und damit weniger Kontrollen möglich.

Dazu wurde erst vor kurzem beim Hygia Project [42] gezeigt, dass nächtliche systolische Hypertonie (erhöhte systolische Blutdruckwerte während der Schlafenszeit, non-dipping, reverse-dipping) den höchsten BD-bedingten Risikofaktor für kardiovaskuläre Ereignisse, Morbidität und Mortalität darstellt. Nachdem der nächtliche BD in der Routine fast nur durch ambulantes 24-h-Blutdruck-Monitoring gemessen werden kann, empfiehlt sich dieses bereits bei Verdacht auf maskierte Hypertonie. Entsprechende Kontrollen sollten bis zum Erreichen der Zielwerte sowie anschließend bei Verdacht auf neuerlich vorliegende nächtliche systolische Hypertonie, non-dipping oder reversedipping durchgeführt werden.

Österreichische Gesellschaft für Hypertensiologie Johann Auer, Mathias C. Brandt, Wolfgang Dichtl, Katharina Hohenstein-Scheibenecker, Sabine Horn, Christian Koppelstätter, Sabine Perl, Friedrich C. Prischl, Miklos Rohla, Jörg Slany, Kurt Stoschitzky, Bruno Watschinger, Thomas Weber, Rene R. Wenzel, Thomas Weiss, David Zweiker, Robert Zweiker

Österreichische Atherosklerosegesellschaft Bernhard Föger, Christoph H. Saely

Österreichische Diabetes Gesellschaft Alexandra KautzkyWiller, Yvonne Winhofer-Stöckl

Österreichische Gesellschaft für Internistische Angiologie Peter Marschang

Österreichische Gesellschaft für Nephrologie Rainer Oberbauer, Gert Mayer, Friedrich C. Prischl

Österreichische Kardiologische Gesellschaft Andrea Podczeck-Schweighofer, Peter Siostrzonek, Bernhard Metzler

Österreichische Gesellschaft für Neurologie Julia Ferrari

Österreichische Schlaganfall-Gesellschaft Julia Ferrari, Michael Knoflach
Österr. Gesellschaft für Allgemeinmedizin Florian Ardelt, Erwin Kepplinger, Florian Obermair, Erwin Rebhandl

Österr. Gesellschaft für Geriatrie Regina Roller-Wirnsberger, Gerhard Wirnsberger

Österreichische Gesellschaft für Endokrinologie und Stoffwechsel Barbara Obermayer-Pietsch, Stefan Pilz

Österreichische Gesellschaft für Innere Medizin Gert Mayer

Österreichische Gesellschaft für Kinder- und Jugendheilkunde Klaus Arbeiter, Christoph Mache, Martin Henkel, Siegfried Waldegger, Christoph Aufricht

Funding Open access funding provided by Medical University of Vienna.

Interessenkonflikt T. Weber: Vortragshonorare und Unterstützung für Kongressbesuche: Servier, Menarini, Novartis, Astra Zeneca, Takeda, Boehringer Ingelheim, MSD, Merck, BOSO, Jacobi Pharma, St Jude, Amgen, medtronic. J. Auer: Vortragshonorare und Unterstützung für Kongressbesuche: Novartis, Astra Zeneca, Bayer, Pfizer, Brisol Myers-Squibb, Daiichi-Sankyo, SanofiTakeda, Boehringer Ingelheim, MSD, Merck, Abbott, Amgen, Medtronic. M. Brandt: Vortragshonorare: Pfizer, Medtronic, Biotronik.W. Dichtl: keine Interessenkonflikte im Zusammenhang mit der Publikation. K. Hohenstein-Scheibenecker: Vortragshonorare und Unterstützung für Kongressbesuche: Novartis, Merck, Eli Lilly, Amgen. S. Horn: Vortragshonorare und Unterstützung für Kongressbesuche: Novartis, Astra Zeneca, Bayer, Pfizer Bayer, Pfizer, Brisol Myers-Squibb, Daiichi-Sankyo, Sanofi, Takeda, Boehringer, MSD, Merck, Abbott, Amgen. C. Koppelstätter: Vortragshonorare: Novartis. S. Perl: Vortragshonorare, Unterstützung für Kongressbesuche, Consulting: Servier, Menarini, Novartis, Astra Zeneca, Takeda, MSD, Merck, BOSO, Sanofi, Neucomed. F. K. Prischl: Honorare für Vorträge oder als advisory board member sowie Kongress- und Tagungseinladungen: Amgen, Astellas, AstraZeneca, Bayer Pharma, Boehringer Ingelheim, Eli Lilly, Evidera, Fresenius Medical Care, Gambro, Janssen-Cilag Pharma, Novartis Pharma, ratiopharm, Roche, Takeda Pharma, Wyeth-Lederle. M. Rohla: Vortrags- und Beraterhonorare von Daiichi Sankyo, Novartis und Takeda Pharma. J. Slany: keine Interessenkonflikte im Zusammenhang mit der Publikation. K. Stoschitzky: keine Interessenkonflikte im Zusammenhang mit der Publikation. B. Watschinger: Vortragshonorare, Unterstützung für Kongressbesuche: Takeda, Gebro, MSD, Servier. R. R. Wenzel: Honorare für Vorträge, Consulting-Tätigkeiten oder wissenschaftliche Projekte: Actelion, Astra-Zeneca, Abbott, Bayer, Daichii-Sankyo, Gepamed, Medtronic, Menarini, Novartis, Speedel Pharma, St Jude Medical, Takeda. T. Weiss: Vortragshonorare und Consulting für Servier, Menarini, Boehringer Ingelheim, medtronic. D. Zweiker: Vortragshonorare: Daiichi Sanchyo. R. Zweiker: Vortragshonorare, Unterstützung für Kongressbesuche, educational grants innerhalb der letzten 12 Monate: Bayer, maw, Sanofi-Aventis, Boehringer-Ingelheim, Gebro, BMS, Abbott, Menarini, Servier, NovoNordisk, Amgen, Daiichi-Sankyo, Novartis. B. Föger: keine Interessenkonflikte im Zusammenhang mit der Publikation. C. H. Saely: Honorare und Forschungsförderungen von Amgen, AstraZeneca, Böhringer-Ingelheim, Lilly, Merck, MSD, Novartis, Novo-Nordisc, Pfizer, Sanofi-Aventis, Takeda. A. KautzkyWiller: Vortragshonorare und Unterstützung für Kongressbesuche: Novo Nordisk, Lilly, Novartis, Sanofi, Astra Zeneca, Boehringer Ingelheim, MSD, Merck, Amgen. Y. WinhoferStöckl: Vortrags- und Beraterhonorare sowie Kongressunterstützung: Amgen, Astra Zeneca, Boehringer Ingelheim, Eli 
Lilly, Merck Sharp \& Dome, Novartis, NovoNordisk, Sanofi Aventis.. P. Marschang: keine Interessenkonflikte im Zusammenhang mit der Publikation. R. Oberbauer: Vortragshonorare und Unterstützung für Kongressbesuche: Novartis, Astra Zeneca, Amgen. G. Mayer: Vortragshonorare für AbbVie, Bayer, Novartis, Böhringer Ingelheim. Novo Nordisk, Amgen, Takeda. A. Podczeck-Schweighofer: Vortragshonorare und Consulting Tätigkeit für Bristol-Myers Squibb, MSD, Daicchi Sankyo, Merck, Medtronic, Sanofi-Aventis. P. Siostrzonek: keine Interesssenkonflikte im Zusammenhang mit der Publikation. B. Metzler: keine Interesssenkonflikte im Zusammenhang mit der Publikation. J. Ferrari: Vortragshonorare und Kongressunterstützung: Böhringer Ingelheim, Sanofi Aventis, Pfizer/BMS, Dajii- Sankyo, Bayer, Novartis, Amgen. M. Knoflach: Vortragshonorare und Kongressunterstützung - Böhringer Ingelheim, Sanofi-Aventis, Daiji-Sankyo.. F. Ardelt: Kongressunterstützung: Novartis. E. Kepplinger: keine Interessenkonflikte im Zusammenhang mit der Publikation. F. Obermair: keine Interessenkonflikte im Zusammenhang mit der Publikation. E. Rebhandl: keine Interessenkonflikte im Zusammenhang mit der Publikation. R. Roller-Wirnsberger: keine Interessenkonflikte im Zusammenhang mit der Publikation. G. Wirnsberger: keine Interessenkonflikte im Zusammenhang mit der Publikation. B. Obermayer-Pietsch: keine Interessenkonflikte im Zusammenhang mit der Publikation. S. Pilz: keine Interessenkonflikte im Zusammenhang mit der Publikation. K. Arbeiter: keine Interessenkonflikte im Zusammenhang mit der Publikation. C. Mache: keine Interessenkonflikte im Zusammenhang mit der Publikation. M. Henkel: keine Interessenkonflikte im Zusammenhang mit der Publikation. S. Waldegger: keine Interessenkonflikte im Zusammenhang mit der Publikation. C. Aufricht: keine Interessenkonflikte im Zusammenhang mit der Publikation.

Open Access Dieser Artikel wird unter der Creative Commons Namensnennung 4.0 International Lizenz (http:// creativecommons.org/licenses/by/4.0/deed.de) veröffentlicht, welche die Nutzung, Vervielfältigung, Bearbeitung, Verbreitung und Wiedergabe in jeglichem Medium und Format erlaubt, sofern Sie den/die ursprünglichen Autor(en) und die Quelle ordnungsgemäß nennen, einen Link zur Creative Commons Lizenz beifügen und angeben, ob Änderungen vorgenommen wurden.

\section{Literatur}

1. Slany JMD, Mayer G, Pichler M, Pilz H, Rieder A, Schernthaner GH, et al. Klassifikation, Diagnostik und Therapie der Hypertonie 2007 -Empfehlungen der Österreichischen Gesellschaft für Hypertensiologie. J Hyperton. 2007;1:7-11.

2. Watschinger BAK, Auer J, Drexel H, Eber B, Fasching P, Grüner P, et al. Klassifikation, Diagnostik, und Therapie der arteriellen Hypertonie 2013: Empfehlungen der Österreichischen Gesellschaft für Hypertensiokogie (ÖGH). JHyperton. 2013;17:99-108.

3. Lim SS, Vos T, Flaxman AD, Danaei G, Shibuya K, AdairRohani H, et al. A comparative risk assessment of burden of disease and injury attributable to 67 risk factors and risk factor clusters in 21 regions, 1990-2010: a systematic analysis for the Global Burden of Disease Study 2010. Lancet. 2012;380:2224-60.

4. Forouzanfar MH, Liu P, Roth GA, Ng M, Biryukov S, MarczakL, etal. GlobalBurden of Hypertension and SystolicBlood Pressure of at Least 110 to $115 \mathrm{~mm} \mathrm{Hg}, 1990-2015$. JAMA. 2017;317:165-82.
5. Rapsomaniki E, Timmis A, George J, Pujades-Rodriguez M, Shah AD, Denaxas S, et al. Blood pressure and incidence of twelve cardiovascular diseases: lifetime risks, healthy life-years lost, and age-specific associations in 1.25 million people. Lancet. 2014;383:1899-911.

6. Lewington S, Clarke R, Qizilbash N, Peto R, Collins R, ProspectiveStudies C.Age-specific relevance of usualblood pressure to vascular mortality: a meta-analysis of individual data for onemillion adults in 61 prospective studies. Lancet. 2002;360:1903-13.

7. Franklin SS, Larson MG, Khan SA, Wong ND, Leip EP, Kannel WB, et al. Does the relation of blood pressure to coronary heart disease risk change with aging? The Framingham Heart Study. Circulation. 2001;103:1245-9.

8. Franklin SS, Khan SA, Wong ND, Larson MG, Levy D. Is pulse pressure useful in predicting risk for coronary heart Disease? The Framingham heart study. Circulation. 1999;100:354-60.

9. Olsen MH, Angell SY, Asma S, Boutouyrie P, Burger D, Chirinos JA, et al. A call to action and a lifecourse strategy to address the global burden of raised blood pressure on current and future generations: the Lancet Commission on hypertension. Lancet. 2016;388:2665-712.

10. Poulter NR, Prabhakaran D, Caulfield M. Hypertension. Lancet. 2015;386:801-12.

11. Thomopoulos C, Parati G, Zanchetti A. Effects of blood pressure lowering on outcome incidence in hypertension. 1. Overview, meta-analyses, and meta-regression analyses of randomized trials. J Hypertens. 2014;32:2285-95.

12. Chow CK, Teo KK, Rangarajan S, IslamS, Gupta R, AvezumA, et al. Prevalence, awareness, treatment, and control of hypertension in rural and urban communities in high-, middle-, and low-income countries. JAMA. 2013;310:959-68.

13. Hitzenberger G, Magometschnigg D. Blood pressure characteristics of hypertensive patients in Austria as determined by self-monitoring (SCREEN-II). Blood Press. 2003;12:134-8.

14. Banegas JR, Lopez-Garcia E, Dallongeville J, Guallar E, Halcox JP, Borghi C, et al. Achievement of treatment goals for primary prevention of cardiovascular disease in clinical practice across Europe: the EURIKA study. Eur Heart J. 2011;32:2143-52.

15. Rohla M, Haberfeld H, Tscharre M, Huber K, Weiss TW. Awareness, treatment, and control of hypertension in Austria: a multicentre cross-sectional study. J Hypertens. 2016;34:1432-40.

16. Danninger KHA, Binder RK, Aichberger M, Hametner B, Wassertheurer S, Weber T. High prevalence of hypertension and early vascular aging: a screening program in pharmacies in Upper Austria. J Hum Hypertens. 2019. https:// doi. org/10.1038/s41371-019-0222-y.

17. Perl S, Zweiker D, Niederl E, Kolesnik E, Zweiker G, Kraler E, et al. May Measurement Month 2017: an analysis of blood pressure screening results in Austria-Europe. Eur Heart J Suppl. 2019;21:D17-D20.

18. Parati G, Ochoa JE, Lombardi C, Bilo G. Blood pressure variability: assessment, predictive value, and potential as a therapeutic target. Curr Hypertens Rep. 2015;17:537.

19. Gratze G, Fortin J, Holler A, Grasenick K, Pfurtscheller G, Wach P, et al. A software package for non-invasive, real-time beat-to-beat monitoring of stroke volume, blood pressure, total peripheral resistanceand for assessment of autonomic function. ComputBiol Med. 1998;28:121-42.

20. Picone DS, Schultz MG, Otahal P, Aakhus S, Al-Jumaily AM, Black JA, et al. Accuracy of cuff-measured blood pressure: systematic reviews and meta-analyses. j Am Coll Cardiol. 2017;70:572-86. 
21. Alpert BS, Quinn D, GallickD. Oscillometric blood pressure: a review for clinicians. JAm Soc Hypertens. 2014;8:930-8.

22. Stergiou GS, Alpert B, Mieke S, Asmar R, Atkins N, Eckert S, et al. A universal standard for the validation of blood pressure measuring devices: Association for the Advancement of Medical Instrumentation/European Society of Hypertension/International Organization for Standardization (AAMI/ESH/ISO) Collaboration Statement. J Hypertens. 2018;36:472-8.

23. Clark CE, Taylor RS, Shore AC, Ukoumunne OC, Campbell JL. Association of a difference in systolic blood pressure between arms with vascular disease and mortality: a systematic review and meta-analysis. Lancet. 2012;379:905-14.

24. Burkard T, Mayr M, Winterhalder C, Leonardi L, Eckstein J, Vischer AS. Reliability of single office blood pressure measurements. Heart. 2018;104:1173-9.

25. Fagard RH, De Cort P. Orthostatic hypotension is a more robust predictor of cardiovascular events than nighttime reverse dipping in elderly. Hypertension. 2010;56:56-61.

26. Myers MG, Kaczorowski J, Dawes M, Godwin M. Automated office blood pressure measurement in primary care. Can Fam Physician. 2014;60:127-32.

27. SPRINT Research Group, Wright JT Jr, Williamson JD, Whelton PK, etal. Arandomized trial of intensive versus standard blood-pressure control. NEngl J Med 2015;373:2103-16.

28. Filipovsky J, Seidlerova J, Kratochvil Z, Karnosova P, Hronova M, Mayer O Jr.. Automated compared to manual office blood pressure and to home blood pressure in hypertensive patients. blood Press. 2016;25:228-34.

29. Wohlfahrt P, Cifkova R, Movsisyan N, Kunzova S, Lesovsky J, Homolka M, et al. Threshold for diagnosing hypertension by automated office blood pressure using random sample population data. JHypertens. 2016;34:2180-6.

30. Myers MG, Godwin M, Dawes M, Kiss A, Tobe SW, Kaczorowski J. Measurement of blood pressure in the office: recognizing the problem and proposing the solution. Hypertension. 2010;55:195-200.

31. Drawz PE, Pajewski NM, Bates JT, Bello NA, Cushman WC, Dwyer JP, et al. Effect of intensive versus standard clinicbased hypertension management on ambulatory blood pressure: results from the SPRINT (systolic blood pressure intervention trial) ambulatory blood pressure study. Hypertension. 2017;69:42-50.

32. Johnson KC, Whelton PK, Cushman WC, Cutler JA, Evans GW, Snyder JK, et al. Blood Pressure Measurement in SPRINT (Systolic Blood Pressure Intervention Trial). Hypertension. 2018;71:848-57.

33. Bliziotis IA, Destounis A, Stergiou GS. Home versus ambulatory and office blood pressure in predicting target organ damage in hypertension: a systematic review and metaanalysis. jHypertens. 2012;30:1289-99.

34. Ward AM, Takahashi O, Stevens R, Heneghan C. Home measurementofblood pressureand cardiovasculardisease: systematic reviewand meta-analysis of prospectivestudies. JHypertens. 2012;30:449-56.

35. McManus RJ, Mant J, Bray EP, Holder R, Jones MI, Greenfield $\mathrm{S}$, et al. Telemonitoring and self-management in the control of hypertension (TASMINH2): a randomised controlled trial. Lancet. 2010;376:163-72.

36. Perl S, Niederl E, Kos C, Mrak P, Ederer H, Rakovac I, et al. Randomized evaluation of the effectiveness of a structured educational program for patients with essential hypertension. Am J Hypertens. 2016;29:866-72.

37. McManus RJ, Mant J, Franssen M, Nickless A, Schwartz C, Hodgkinson J, et al. Efficacy of self-monitored blood pressure, with or without telemonitoring, for titration of antihy- pertensive medication (TASMINH4): an unmasked randomised controlled trial. Lancet. 2018;391:949-59.

38. McManus RJ, Mant J, Haque MS, Bray EP, Bryan S, Greenfield SM, et al. Effect of self-monitoring and medication self-titration on systolic blood pressure in hypertensive patients at high risk of cardiovascular disease: the TASMIN-SR randomized clinical trial. JAMA. 2014;312:799-808.

39. Zweiker R, Slany J. Zeitgemäße Hochdruckdiagnose I: Ambulantes 24-Stunden-Blutdruckmonitoring. J Hyperton. 2012;16:18-24.

40. Gaborieau V, Delarche N, Gosse P. Ambulatory blood pressuremonitoringversusself-measurement ofblood pressure athome: correlation with target organ damage.J Hypertens. 2008;26:1919-27.

41. Banegas JR, Ruilope LM, de la Sierra A, Vinyoles E, Gorostidi M, de la Cruz JJ, et al. Relationship between Clinic and Ambulatory Blood-Pressure Measurements and Mortality. NEngl J Med. 2018;378:1509-20.

42. Hermida RC, Crespo JJ, Otero A, Dominguez-Sardina M, Moya A, Rios MT, et al. Asleep blood pressure: significant prognostic marker of vascular risk and therapeutic target for prevention. Eur Heart J. 2018;39:4159-71.

43. Parati G, Stergiou G, O’Brien E, Asmar R, Beilin L, Bilo G, et al. European Society of Hypertension practice guidelines for ambulatory blood pressure monitoring. J Hypertens. 2014;32:1359-66.

44. Mancia G, Verdecchia P. Clinical value of ambulatory blood pressure: evidence and limits. Circ Res. 2015;116:1034-45.

45. Sharman JE, LaGerche A. Exercise blood pressure: clinical relevance and correct measurement. J Hum Hypertens. 2015;29:351-8.

46. Caselli S, Serdoz A, Mango F, Lemme E, Vaquer Segui A, Milan A, et al. High blood pressure response to exercise predicts future development of hypertension in young athletes. Eur Heart J. 2019;40:62-8.

47. Mancia G, Bombelli M, Cuspidi C, Facchetti R, Grassi G. Cardiovascular risk associated with white-coat hypertension: pro side of the argument. Hypertension. 2017;70:668-75.

48. Berry JD, Dyer A, Cai X, Garside DB, Ning H, Thomas A, et al. Lifetime risks of cardiovascular disease. N Engl J Med. 2012;366:321-9.

49. Piepoli MF, Hoes AW, Agewall S, Albus C, Brotons C, Catapano AL, et al. 2016 European Guidelines on cardiovascular disease prevention in clinical practice: The Sixth Joint Task Force of the European Society of Cardiology and Other Societies on Cardiovascular Disease Prevention in Clinical Practice (constituted by representatives of 10 societies and by invited experts)Developed with the special contribution of the European Association for Cardiovascular Prevention \& Rehabilitation (EACPR). Eur HeartJ.2016;37:2315-81.

50. Perk J, De Backer G, Gohlke H, Graham I, Reiner Z, Verschuren M, et al. European Guidelines on cardiovascular disease prevention in clinical practice (version 2012). The Fifth Joint Task Force of the European Society of Cardiology and Other Societies on Cardiovascular Disease Prevention in Clinical Practice (constituted by representatives of nine societies and by invited experts). Eur Heart J.2012;33:1635-701.

51. Belcaro G, Nicolaides AN, Ramaswami G, Cesarone MR, De Sanctis M, IncandelaL, etal.Carotidandfemoralultrasound morphology screening and cardiovascular events in low risk subjects: a 10-year follow-up study (the CAFES-CAVE study(1)).Atherosclerosis. 2001;156:379-87.

52. Williams B, Mancia G, Spiering W, Agabiti Rosei E, Azizi M, Burnier M, et al. 2018a ESC/ESH Guidelines for the management of arterial hypertension. Eur Heart J. 2018;39:3021-104. 
53. Schmieder RE. End organ damage in hypertension. Dtsch Arztebl Int. 2010;107:866-73.

54. Devereux RB, Alderman MH. Role of preclinical cardiovascular disease in the evolution from risk factor exposure to development of morbid events. Circulation. 1993;88:1444-55.

55. Greve SV, Blicher MK, Sehestedt T, Gram-Kampmann EM, Rasmussen S, Vishram JK, et al. Effective risk stratification in patients with moderate cardiovascular risk using albuminuria and atherosclerotic plaques in the carotid arteries. JHypertens. 2015;33:1563-70.

56. Sehestedt T, Jeppesen J, Hansen TW, Wachtell K, Ibsen H, Torp-Pedersen C, et al. Risk prediction is improved by adding markers of subclinical organ damage to SCORE. Eur Heart J. 2010;31:883-91.

57. Lonnebakken MT, Izzo R, Mancusi C, Gerdts E, Losi MA, Canciello $\mathrm{G}$, et al. Left ventricular hypertrophy regression during antihypertensive treatment in an outpatient clinic (the Campania salute network). JAHA. 2017; https://doi. org/10.1161/JAHA.116.004152.

58. Pierdomenico SD, Cuccurullo F. Risk reduction after regression of echocardiographic left ventricular hypertrophy in hypertension: a meta-analysis. Am J Hypertens. 2010;23:876-81.

59. Ibsen H, Olsen MH, Wachtell K, Borch-Johnsen K, Lindholm LH, Mogensen CE, et al. Reduction in albuminuria translates to reduction in cardiovascular events in hypertensive patients: losartan intervention for endpoint reduction in hypertension study. Hypertension. 2005;45:198-202.

60. Dichtl W. Echokardiographie in der Abklärung der Hypertonie. J Hyperton. 2016;40:94-7.

61. Marwick TH, Gillebert TC, Aurigemma G, Chirinos J, Derumeaux $G$, Galderisi $M$, et al. Recommendations on the use of echocardiography in adult hypertension: a report from the European Association of Cardiovascular Imaging (EACVI) and the American Society of Echocardiography (ASE)dagger. Eur Heart J Cardiovasc Imaging. 2015;16:577-605.

62. Nambi V, Chambless L, Folsom AR, He M, Hu Y, Mosley T, et al.Carotidintima-mediathickness and presence or absence of plaque improves prediction of coronary heart disease risk: the ARIC (Atherosclerosis Risk In Communities) study. JAm Coll Cardiol. 2010;55:1600-7.

63. Van Bortel LM, Laurent S, Boutouyrie P, Chowienczyk P, CruickshankJK, De Backer T, et al. Function and European Network for Noninvasive Investigation of Large A. Expert consensus document on the measurement of aortic stiffness in daily practice using carotid-femoral pulse wave velocity.j Hypertens. 2012;30:445-8.

64. Vlachopoulos C, Xaplanteris P, Aboyans V, Brodmann M, Cifkova R, Cosentino F, et al. The role of vascular biomarkers for primary and secondary prevention. A position paper from the European Society of Cardiology Working Group on peripheral circulation: Endorsed by the Association for Research into Arterial Structure and Physiology (ARTERY) Society. Atherosclerosis. 2015;241:507-32.

65. Guerin AP, Blacher J, Pannier B, Marchais SJ, Safar ME, London GM. Impact of aortic stiffness attenuation on survival of patients in end-stage renal failure. Circulation. 2001;103:987-92.

66. Weber T, Wassertheurer S, Hametner B, Parragh S, Eber B. Noninvasive methods to assess pulse wave velocity: comparison with the invasive gold standard and relationship with organ damage. jHypertens. 2015;33:1023-31.

67. Aboyans V, RiccoJB, BartelinkMEL, BjorckM, Brodmann M, Cohnert T, et al. 2017 ESC Guidelines on the Diagnosis and Treatment of Peripheral Arterial Diseases, in collaboration with the European Society for Vascular Surgery (ESVS): Document covering atherosclerotic disease of extracranial carotid and vertebral, mesenteric, renal, upper and lower extremity arteriesEndorsed by: the European Stroke Organization (ESO)The Task Force for the Diagnosis and Treatment of Peripheral Arterial Diseases of the European Society of Cardiology (ESC) and of the European Society for Vascular Surgery (ESVS). Eur Heart J. 2018;39:763-816.

68. Levey AS, Stevens LA, Schmid CH, Zhang YL, Castro AF 3rd, Feldman HI, et al. A new equation to estimate glomerular filtration rate. Ann Intern Med. 2009;150:604-12.

69. McTaggart MP, Newall RG, Hirst JA, Bankhead CR, Lamb EJ, Roberts NW, etal. Diagnostic accuracy of point-of-care tests for detecting albuminuria: a systematic review and metaanalysis. Ann Intern Med. 2014;160:550-7.

70. Gerstein HC, Mann JF, Yi Q, Zinman B, Dinneen SF, Hoogwerf $\mathrm{B}$, et al. Albuminuria and risk of cardiovascular events, death, and heart failure in diabetic and nondiabetic individuals. JAMA. 2001;286:421-6.

71. Breslin DJ, Gifford RW Jr., Fairbairn JF 2nd, Kearns TP. Prognostic importance of ophthalmoscopic findings in essential hypertension. JAMA. 1966;195:335-8.

72. Iadecola C, Yaffe K, Biller J, Bratzke LC, Faraci FM, Gorelick $\mathrm{PB}$, et al. Impact of hypertension on cognitive function: a scientific statementfrom the American Heart Association. Hypertension. 2016;68:e67-e94.

73. Kearney-Schwartz A, Rossignol P, Bracard S, Felblinger J, Fay R, Boivin JM, et al. Vascular structure and function is correlated to cognitive performance and white matter hyperintensities in older hypertensive patients with subjective memory complaints. Stroke. 2009;40:1229-36.

74. Mitchell GF, van Buchem MA, Sigurdsson S, Gotal JD, Jonsdottir MK, Kjartansson O, et al. Arterial stiffness, pressure and flow pulsatility and brain structure and function: the Age, Gene/Environment Susceptibility-Reykjavik study. Brain. 2011;134:3398-407.

75. Dominiczak A, Delles C, Padmanabhan S. Genomics and precision medicine for clinicians and scientists in hypertension. Hypertension. 2017;69:e10-e3.

76. Grossman A, Messerli FH, Grossman E. Drug induced hypertension-An unappreciated cause of secondary hypertension. EurJPharmacol.2015;763:15-22.

77. Rimoldi SF, Scherrer U, Messerli FH. Secondary arterial hypertension: when, who, and how to screen? Eur Heart J. 2014;35:1245-54.

78. Lassnig E, Weber T, Auer J, Nomeyer R, Eber B. Pheochromocytoma crisis presenting with shock and tako-tsubo-like cardiomyopathy. Int J Cardiol. 2009;134:e138-e40.

79. PrischlFC, Seiringer E, Wallner M, Wimmer L, Windpessl M. Renal artery stenosis and ischemic kidney disease-a review. JHyperton. 2017;21:5-12.

80. Murphy TP, Soares G, Kim M. Increase in utilization of percutaneous renal artery interventions by medicare beneficiaries, 1996-2000. AJR Am J Roentgenol. 2004;183:561-8.

81. Textor SC. Atherosclerotic renal artery stenosis: overtreated but underrated? J Am Soc Nephrol. 2008;19:656-9.

82. Rihal CS, Textor SC, Breen JF, McKusick MA, Grill DE, Hallett JW, et al. Incidental renal artery stenosis among a prospective cohort of hypertensive patients undergoing coronary angiography. Mayo Clin Proc. 2002;77:309-16.

83. MesserliFH, BangaloreS, MakaniH, RimoldiSF, AllemannY, White CJ, et al. Flash pulmonary oedema and bilateral renal artery stenosis: the Pickering syndrome. Eur Heart J. 2011;32:2231-5.

84. TextorS, MaillouxLU.Clinicalmanifestations and diagnosis of chronic kidney disease resulting from atherosclerotic renal artery stenosis. In: UpToDate 2019; (Literature review 
current through September 2019. Last updated May 10, 2018). Last accessed via www.uptodate.com October 31, 2019.

85. Hirsch AT, Haskal ZJ, Hertzer NR, Bakal CW, Creager MA, Halperin JL, et al. ACC/AHA 2005 guidelines for the management of patients with peripheral arterial disease (lower extremity, renal, mesenteric, and abdominal aortic): executive summary a collaborative report from the American Association for Vascular Surgery/Society for Vascular Surgery, Society for Cardiovascular Angiography and Interventions, Society for Vascular Medicine and Biology, Society of Interventional Radiology, and the ACC/AHA Task Force on Practice Guidelines (Writing Committee to Develop Guidelines for the Management of Patients With Peripheral Arterial Disease) endorsed by the American Association of Cardiovascular and Pulmonary Rehabilitation; National Heart, Lung, and Blood Institute; Society for Vascular Nursing; TransAtlantic Inter-Society Consensus; and Vascular Disease Foundation. J Am Coll Cardiol. 2006;47:1239-312.

86. Jennings CG, Houston JG, Severn A, Bell S, Mackenzie IS, Macdonald TM. Renal artery stenosis-when to screen, what to stent? Curr Atheroscler Rep. 2014;16:416.

87. Zeller T, Bonvini RF, Sixt S. Color-coded duplex ultrasound for diagnosis of renal artery stenosis and as follow-up examination after revascularization. Catheter Cardiovasc Interv. 2008;71:995-9.

88. Williams GJ, Macaskill P, Chan SF, Karplus TE, Yung W, Hodson EM, et al. Comparative accuracy of renal duplex sonographic parameters in the diagnosis of renal artery stenosis: paired and unpaired analysis. AJR Am J Roentgenol. 2007;188:798-811.

89. Radermacher J, Chavan A, Bleck J, Vitzthum A, Stoess B, Gebel MJ, et al. Use of Doppler ultrasonography to predict the outcome of therapy for renal-artery stenosis. N Engl J Med. 2001;344:410-7.

90. Postma CT, Joosten FB, Rosenbusch G, Thien T. Magnetic resonanceangiographyhasahighreliabilityin the detection of renal artery stenosis. Am J Hypertens. 1997;10:957-63.

91. Olbricht CJ, Galanski M, Chavan A, Prokop M. Spiral CT angiography-can we forget about arteriography to diagnose renal artery stenosis? Nephrol Dial Transplant. 1996;11:1227-31.

92. Tan KT, van Beek EJ, Brown PW, van Delden OM, Tijssen J, Ramsay LE. Magnetic resonance angiography for the diagnosis of renal artery stenosis: a meta-analysis. Clin Radiol. 2002;57:617-24.

93. AbuRahma AF, Yacoub M. Renal imaging: duplex ultrasound, computed tomography angiography, magnetic resonance angiography, and angiography. Semin Vasc Surg. 2013;26:134-43.

94. De Bruyne B, Manoharan G, Pijls NH, Verhamme K, Madaric J, Bartunek J, et al. Assessment of renal artery stenosis severity by pressure gradient measurements. J Am Coll Cardiol. 2006;48:1851-5.

95. Bommart S, Cliche A, Therasse E, Giroux MF, Vidal V, Oliva VL, et al. Renal artery revascularization: predictive value of kidney length and volume weighted by resistive index. AJRAm J Roentgenol.2010;194:1365-72.

96. Modrall JG, Rosero EB, Leonard D, Timaran CH, Anthony T, Arko FA 3rd, et al. Clinical and kidney morphologic predictors of outcome for renal artery stenting: data to inform patient selection. J Vasc Surg. 2011;53:1282-9. discussion 1289-90.

97. Conlon PJ, Little MA, Pieper K, Mark DB. Severity of renal vascular disease predicts mortality in patients undergoing coronary angiography. Kidney Int. 2001;60:1490-7.
98. Mailloux LU, Napolitano B, Bellucci AG, Vernace M, Wilkes BM, Mossey RT. Renal vascular disease causing endstage renal disease, incidence, clinical correlates, and outcomes: a 20-year clinical experience. Am J Kidney Dis. 1994;24:622-9.

99. Catapano AL, Graham I, De Backer G, Wiklund O, Chapman MJ, Drexel H, et al. 2016 ESC/EAS guidelines for the management of dyslipidaemias. Eur Heart J. 2016;37:2999-3058.

100. Mancia G, Fagard R, Narkiewicz K, Redon J, Zanchetti A, Bohm M, et al. $2013 \mathrm{ESH} / \mathrm{ESC}$ Guidelines for the management of arterial hypertension: the Task Force for the management of arterial hypertension of the European Society of Hypertension (ESH) and of the European Society of Cardiology (ESC). JHypertens. 2013;31:1281-357.

101. Toplak H, Ludvik B, Lechleitner M, Dieplinger H, Foger B, Paulweber B, et al. Austrian Lipid Consensus on the management of metabolic lipid disorders to prevent vascular complications: A joint position statement issued by eight medical societies. 2016 update. Wien Klin Wochenschr. 2016;128(Suppl2):S216-S28.

102. Evans KL, Tuttle KR, Folt DA, Dawson T, Haller ST, Brewster PS, et al. Use of renin-angiotensin inhibitors in people with renal artery stenosis. Clin J Am Soc Nephrol. 2014;9:1199-206.

103. Hackam DG, Duong-Hua ML, Mamdani M, Li P, Tobe SW, Spence JD, et al. Angiotensin inhibition in renovascular disease: a population-based cohort study. Am Heart J. 2008;156:549-55.

104. Cooper CJ, Murphy TP, Cutlip DE, Jamerson K, Henrich W, Reid DM, et al. Stenting and medical therapy for atherosclerotic renal-artery stenosis. NEnglJ Med. 2014;370:13-22.

105. ASTRAL Investigators. Wheatley K, Ives N, Gray R, et al. Revascularization versus medical therapy for renal artery stenosis. NEngl J Med.2009;361(20):1953-62.

106. Bax L, Woittiez AJ, Kouwenberg HJ, Mali WP, Buskens E, BeekFJ, etal.Stent placement in patients with atherosclerotic renal artery stenosis and impaired renal function: a randomized trial.Ann Intern Med.2009;150(840-8):W150-W1.

107. Herrmann SM, Saad A, Textor SC. Management of atherosclerotic renovascular disease after Cardiovascular Outcomes in Renal Atherosclerotic Lesions (CORAL). Nephrol Dial Transplant. 2015;30:366-75.

108. Ritchie J, Green D, Chrysochou C, Chalmers N, Foley RN, Kalra PA. High-risk clinical presentations in atherosclerotic renovascular disease: prognosis and response to renal artery revascularization. Am J Kidney Dis. 2014;63:186-97.

109. Vassallo D, Ritchie J, Green D, Chrysochou C, Kalra PA. The effect of revascularization in patients with anatomically significant atherosclerotic renovascular disease presenting with high-risk clinical features. Nephrol Dial Transplant. 2018;33:497-506.

110. Kane GC, Xu N, Mistrik E, Roubicek T, Stanson AW, Garovic VD. Renal artery revascularization improves heart failure control in patients with atherosclerotic renal artery stenosis. Nephrol Dial Transplant. 2010;25:813-20.

111. Persu A, Giavarini A, Touzé E, Januszewicz A, Sapoval M, Azizi M, Barral X, Jeunemaitre X, Morganti A, Plouin PF, de LeeuwP; ESHWorking Group Hypertension and the Kidney. European consensus on the diagnosis and management of fibromuscular dysplasia. J Hypertens. 2014;32:1367-78.

112. Mousa AY, Campbell JE, Stone PA, Broce M, Bates MC, AbuRahma AF. Short- and long-term outcomes of percutaneous transluminal angioplasty/stenting of renal fibromuscular dysplasia over a ten-year period. J Vasc Surg. 2012;55:421-7.

113. Trinquart L, Mounier-Vehier C, Sapoval M, Gagnon N, Plouin PF. Efficacy of revascularization for renal artery 
stenosis caused by fibromuscular dysplasia: a systematic review and meta-analysis. Hypertension. 2010;56:525-32.

114. Lechner B, Heinrich D, Nolting S, Osswald-Kopp A, Rubinstein G, Sauerbeck J, et al. Update on endocrine hypertension. Internist. 2018;59:1163-79.

115. Pappachan JM, Buch HN. Endocrine hypertension: a practical approach. Adv Exp Med Biol.2016;956:215-37.

116. Whelton PK, Carey RM, Aronow WS, Casey DE Jr., Collins KJ, Dennison Himmelfarb C, etal. 2017 ACC/AHA/AAPA/ABC/ ACPM/AGS/APhA/ASH/ASPC/NMA/PCNA Guideline for the Prevention, Detection, Evaluation, and Management of High Blood Pressure in Adults: A Report of the American College of Cardiology/American Heart Association Task Force on Clinical Practice Guidelines. Circulation. 2018;138:e484-e594.

117. Young WF, Calhoun DA, Lenders JWM, Stowasser M, TextorSC.Screening for endocrinehypertension: an endocrine society scientific statement. Endocr Rev. 2017;38:103-22.

118. Funder JW, Carey RM, Mantero F, Murad MH, Reincke M, Shibata $\mathrm{H}$, et al. The management of primary aldosteronism: case detection, diagnosis, and treatment: an endocrine society clinical practice guideline. J Clin Endocrinol Metab. 2016;101:1889-916.

119. Pilz S, Tomaschitz A, Trummer C, Bachmann A, Pandis M, Schwetz V, et al. Primärer Hyperaldosteronismus: Diagnostikund Therapie. J Klin Endokrinol Stoffw. 2017;10:82-91.

120. Vaidya A, Mulatero P, Baudrand R, Adler GK. The expanding spectrum of primary aldosteronism: implications for diagnosis, pathogenesis, and treatment. Endocr Rev. 2018;39:1057-88.

121. Young WF Jr.. Diagnosis and treatment of primary aldosteronism: practical clinical perspectives. J Intern Med. 2019;285:126-48.

122. Lenders JW, Duh QY, Eisenhofer G, Gimenez-Roqueplo AP, Grebe SK, Murad MH, et al. Pheochromocytoma and paraganglioma: an endocrine society clinical practice guideline. JClin Endocrinol Metab. 2014;99:1915-42.

123. Pilz S. Nebenniereninsuffizienz und Nebenniereninzidentalome. Wien Klin Wochenschr Educ. 2017;12:81-101.

124. Williams B, Mancia G, Spiering W, Agabiti Rosei E, Azizi M, Burnier M, etal.2018bESC/ESH Guidelinesfor themanagement of arterial hypertension: the Task Force for the management of arterial hypertension of the European Society of Cardiology and the European Society of Hypertension: The TaskForcefor the management of arterial hypertension of the European Society of Cardiology and the European Society of Hypertension. J Hypertens. 2018;36:1953-2041.

125. Monticone S, D’Ascenzo F, Moretti C, Williams TA, Veglio F, GaitaF, etal.Cardiovascular events and targetorgan damage in primary aldosteronism compared with essential hypertension: a systematic review and meta-analysis. Lancet Diabetes Endocrinol. 2018;6:41-50.

126. WillenbergHS. How to escape from primaryaldosteronism? News and views on an adrenal disorder of salt retention. Horm Metab Res. 2017;49:151-63.

127. Brown MJ, Drake WM. Primary aldosteronism as a public health issue-Authors' reply. Lancet Diabetes Endocrinol. 2016;4:973-4.

128. Buffolo F, Monticone S, Burrello J, Tetti M, Veglio F, Williams TA, et al. Is primary aldosteronism still largely unrecognized? Horm Metab Res. 2017;49:908-14.

129. Funder JW. Primary aldosteronism as a public health issue. LancetDiabetes Endocrinol. 2016;4:972-3.

130. Manolopoulou J, Fischer E, Dietz A, Diederich S, Holmes D, Junnila R, et al. Clinical validation for the aldosteroneto-renin ratio and aldosterone suppression testing using simultaneous fully automated chemiluminescence immunoassays. J Hypertens. 2015;33:2500-11.

131. Dekkers T, Prejbisz A, Kool LJS, Groenewoud H, Velema M, Spiering W, et al. Adrenal vein sampling versus CT scan to determine treatment in primary aldosteronism: an outcome-based randomised diagnostic trial. Lancet Diabetes Endocrinol. 2016;4:739-46.

132. Williams B, MacDonald TM, Morant SV, Webb DJ, Sever P, McInnes GT, et al. Endocrine and haemodynamic changes in resistant hypertension, and blood pressure responses to spironolactone or amiloride: the PATHWAY-2 mechanisms substudies. Lancet Diabetes Endocrinol. 2018;6:464-75.

133. Hundemer GL, Curhan GC, Yozamp N, Wang M, Vaidya A. Cardiometabolic outcomes and mortality in medically treated primary aldosteronism: a retrospective cohort study. LancetDiabetes Endocrinol. 2018;6:51-9.

134. Williams TA, Lenders JWM, Mulatero P, Burrello J, Rottenkolber M, Adolf C, et al. Outcomes after adrenalectomy for unilateral primary aldosteronism: an international consensus on outcome measures and analysis of remission rates in an international cohort. Lancet Diabetes Endocrinol. 2017;5:689-99.

135. Gruber M, Darr R, Eisenhofer G. Pheochromocytoma: update on diagnosis and therapy. Dtsch Med Wochenschr. 2014;139:486-90.

136. Neary NM, King KS, Pacak K. Drugs and pheochromocytoma-don't be fooled by every elevated metanephrine. NEnglJ Med. 2011;364:2268-70.

137. Nieman LK. Diagnosis of cushing's syndromein the modern era. Endocrinol Metab Clin North Am. 2018;47:259-73.

138. Nieman LK, Biller BM, Findling JW, Newell-Price J, Savage MO, Stewart PM, et al. The diagnosis of cushing's syndrome: an endocrine society clinical practice guideline. JClin Endocrinol Metab. 2008;93:1526-40.

139. Nieman LK, Biller BM, Findling JW, Murad MH, NewellPrice J, Savage MO, et al. Treatment of cushing's syndrome: an endocrine society clinical practice guideline. j Clin Endocrinol Metab. 2015;100:2807-31.

140. Pivonello R, De Leo M, Cozzolino A, Colao A. The treatment of cushing's disease. Endocr Rev. 2015;36:385-486.

141. Valassi E, Franz H, Brue T, Feelders RA, Netea-Maier R, Tsagarakis S, et al. Diagnostic tests for Cushing's syndrome differ from published guidelines: data from ERCUSYN.Eur J Endocrinol. 2017;176:613-24.

142. Melcescu E, Phillips J, Moll G, Subauste JS, Koch CA. 11Betahydroxylase deficiency and other syndromes of mineralocorticoid excess as a rare cause of endocrine hypertension. Horm Metab Res. 2012;44:867-78.

143. Nicolaides NC, Charmandari E. Chrousos syndrome: from molecular pathogenesis to therapeutic management. Eur J Clin Invest. 2015;45:504-14.

144. Funder JW. Apparent mineralocorticoid excess. J Steroid Biochem Mol Biol. 2017;165:151-3.

145. Razzaghy-Azar M, Yau M, Khattab A, New MI. Apparent mineralocorticoid excess and the long term treatment of genetic hypertension. J Steroid Biochem Mol Biol. 2017;165:145-50.

146. Schwetz V, Aberer F, Stiegler C, Pieber RT, ObermayerPietsch B, Pilz S. Fluconazole and acetazolamide in the treatment of ectopic Cushing's syndrome with severe metabolic alkalosis. endocrinol Diabetes Metab Case Rep. 2015;2015:150027.

147. Baudrand R, Vaidya A. The low-Renin hypertension phenotype: genetics and the role of the mineralocorticoid receptor. Int J Mol Sci. 2018; https://doi.org/10.3390/ ijms19020546. 
148. Mumford E, Unwin RJ, Walsh SB. Liquorice, Liddle, Bartter or Gitelman-how to differentiate? Nephrol Dial Transplant. 2019;34:38-9.

149. Tetti M, Monticone S, Burrello J, Matarazzo P, Veglio F, Pasini B, et al. Liddle syndrome: review of the literature and description of a new case. IntJMolSci. 2018;19:812.

150. Bilezikian JP, Bandeira L, Khan A, Cusano NE. Hyperparathyroidism. Lancet. 2018;391:168-78.

151. Bilezikian JP, Brandi ML, Eastell R, Silverberg SJ, Udelsman R, Marcocci C, et al. Guidelines for the management of asymptomatic primary hyperparathyroidism: summary statement from the Fourth International Workshop. J Clin Endocrinol Metab. 2014;99:3561-9.

152. Pepe J, Cipriani C, Sonato C, Raimo O, Biamonte F, Minisola S. Cardiovascular manifestations of primary hyperparathyroidism: a narrative review. Eur J Endocrinol. 2017;177:R297-R308.

153. Katznelson L, Laws ER Jr., Melmed S, Molitch ME, Mu$\operatorname{rad} \mathrm{MH}, \mathrm{Utz} \mathrm{A}$, et al. Acromegaly: an endocrine society clinical practice guideline. J Clin Endocrinol Metab. 2014;99:3933-51.

154. Melmed S, Bronstein MD, Chanson P, Klibanski A, Casanueva FF, Wass JAH, et al. A Consensus Statement on acromegaly therapeutic outcomes. Nat Rev Endocrinol. 2018;14:552-61.

155. Ramos-Levi AM, Marazuela M. Cardiovascular comorbidities in acromegaly: an update on their diagnosis and management. Endocrine. 2017;55:346-59.

156. Lavrentaki A, Paluzzi A, Wass JA, Karavitaki N. Epidemiology of acromegaly: review of population studies. Pituitary. 2017;20:4-9.

157. Textor SC. Renal arterial disease and hypertension. Med Clin North Am. 2017;101:65-79.

158. Gu WJ, Zhang LX, Jin N, Ba JM, Dong J, Wang DJ, et al. Rare and curable renin-mediated hypertension: a series of six cases and a literature review. J Pediatr Endocrinol Metab. 2016;29:209-16.

159. Trnka P, Orellana L, Walsh M, Pool L, Borzi P. Reninoma: an uncommon cause of Renin-mediated hypertension. Front Pediatr. 2014;2:89.

160. Delitala AP. Subclinical hyperthyroidism and the cardiovascular disease. Horm Metab Res. 2017;49:723-31.

161. Grais IM, Sowers JR. Thyroid and the heart. Am J Med. 2014;127:691-8.

162. He W, Li S, Zhang JA, Zhang J, Mu K, Li XM. Effect of levothyroxine on blood pressure in patients with subclinical hypothyroidism: a systematic review and meta-analysis. FrontEndocrinol. 2018;9:454.

163. Ichiki T. Thyroid hormone and vascular remodeling. J Atheroscler Thromb. 2016;23:266-75.

164. Jonklaas J, Bianco AC, Bauer AJ, Burman KD, Cappola AR, Celi FS, et al. Guidelines for the treatment of hypothyroidism: prepared by the American Thyroid Association task force on thyroid hormone replacement. Thyroid. 2014;24:1670-751.

165. Kahaly GJ, Bartalena L, Hegedus L, Leenhardt L, Poppe K, PearceSH.2018EuropeanThyroidAssociationguidelinefor the management of graves' hyperthyroidism. Eur Thyroid J. 2018;7:167-86.

166. Pearce SH, Brabant G, Duntas LH, Monzani F, Peeters RP, Razvi S, et al. 2013 ETA guideline: management of subclinical hypothyroidism. Eur Thyroid J.2013;2:215-28.

167. Ross DS, Burch HB, Cooper DS, Greenlee MC, Laurberg P, Maia AL, et al. 2016 American Thyroid Association guidelines for diagnosis and management of hyperthyroidism and other causes of thyrotoxicosis. Thyroid. 2016;26:1343-421.
168. Stevens VJ, Obarzanek E, Cook NR, Lee IM, Appel LJ, Smith West D, et al. Long-term weight loss and changes in blood pressure: results of the Trials of Hypertension Prevention, phase II. Ann Intern Med.2001;134:1-11.

169. Elliott P, Stamler J, Nichols R, Dyer AR, Stamler R, Kesteloot $\mathrm{H}$, et al. Intersalt revisited: further analyses of 24 hour sodium excretion and blood pressure within and across populations. Intersalt Cooperative Research Group. BMJ. 1996;312:1249-53.

170. He FJ, Li J, Macgregor GA. Effect of longer term modest salt reduction on blood pressure: Cochrane systematic review and meta-analysis of randomised trials. BMJ. 2013;346:1325.

171. Suckling RJ, He FJ, Markandu ND, MacGregor GA. Modest salt reduction lowers blood pressure and albumin excretion in impaired glucose tolerance and type 2 diabetes mellitus: a randomized double-blind trial. Hypertension. 2016;67:1189-95.

172. Appel LJ, Espeland MA, Easter L, Wilson AC, Folmar S, Lacy CR. Effects of reduced sodium intake on hypertension control in older individuals: results from the trial of nonpharmacologic interventions in the elderly (TONE). Arch Intern Med.2001;161:685-93.

173. Huggins CE, Margerison C, Worsley A, Nowson CA. Influence of dietary modifications on the blood pressure response to antihypertensive medication. $\mathrm{Br} \mathrm{J}$ Nutr. 2011;105:248-55.

174. Pimenta E, Gaddam KK, Oparil S, Aban I, Husain S, Dell'Italia LJ, et al. Effects of dietary sodium reduction on blood pressure in subjects with resistant hypertension: results from a randomized trial. Hypertension. 2009;54:475-81.

175. Appel LJ, Moore TJ, Obarzanek E, Vollmer WM, Svetkey LP, Sacks FM, et al. A clinical trial of the effects of dietary patterns on blood pressure. DASH Collaborative Research Group. NEngl J Med. 1997;336:1117-24.

176. Estruch R, Ros E, Salas-Salvado J, Covas MI, Corella D, Aros F, et al. Retraction and republication: primary prevention of cardiovascular disease with a mediterranean diet. N Engl J Med. 2013;368:1279-90. The New England journal of medicine. 2018;378:2441-2442.

177. Domenech M, Roman P, Lapetra J, Garcia de la Corte FJ, Sala-Vila A, de la Torre R, et al. Mediterranean diet reduces 24-hour ambulatory blood pressure, blood glucose, and lipids: one-year randomized, clinical trial. Hypertension. 2014;64:69-76.

178. Vlachopoulos C, Hirata K, Stefanadis C, Toutouzas P, O'Rourke MF. Caffeine increases aortic stiffness in hypertensive patients. Am J Hypertens. 2003;16:63-6.

179. Ding M, Bhupathiraju SN, Satija A, van Dam RM, Hu FB. Long-term coffee consumption and risk of cardiovascular disease: a systematic review and a dose-response meta-analysis of prospective cohort studies. Circulation. 2014;129:643-59.

180. Greyling A, Ras RT, Zock PL, Lorenz M, Hopman MT, Thijssen DH, et al. The effect of black tea on blood pressure: a systematic review with meta-analysis of randomized controlled trials. Plos One. 2014;9:e103247.

181. Li G, Zhang Y, Thabane L, Mbuagbaw L, Liu A, Levine MA, et al. Effect of green tea supplementation on blood pressure among overweight and obese adults: a systematic review and meta-analysis. J Hypertens. 2015;33:243-54.

182. Whelton PK, He J, Cutler JA, Brancati FL, Appel LJ, Follmann D, et al. Effects of oral potassium on blood pressure. Meta-analysis of randomized controlled clinical trials. JAMA. 1997;277:1624-32. 
183. Bazzano LA,HeJ, Ogden LG, LoriaCM, VupputuriS, Myers L, et al. Fruit and vegetable intake and risk of cardiovascular disease in US adults: the first national health and nutrition examination survey epidemiologic follow-up study. Am J Clin Nutr. 2002;76:93-9.

184. Ascherio A, Rimm EB, Giovannucci EL, Colditz GA, Rosner B, Willett WC, et al. A prospective study of nutritional factors and hypertension among US men. Circulation. 1992;86:1475-84.

185. Forman JP, Stampfer MJ, Curhan GC. Diet and lifestyle risk factors associated with incident hypertension in women. JAMA. 2009;302:401-11.

186. Tuck ML, Sowers J, Dornfeld L, Kledzik G, Maxwell M. The effect of weight reduction on blood pressure, plasma renin activity, and plasma aldosterone levels in obese patients. NEngl J Med. 1981;304:930-3.

187. Neter JE, Stam BE, Kok FJ, Grobbee DE, Geleijnse JM. Influence of weight reduction on blood pressure: a metaanalysis of randomized controlled trials. Hypertension. 2003:42:878-84.

188. Whelton PK, Appel LJ, Espeland MA, Applegate WB, Ettinger $\mathrm{WH}$ Jr., Kostis JB, et al. Sodium reduction and weight loss in the treatment of hypertension in older persons: a randomized controlled trial of nonpharmacologic interventions in the elderly (TONE). TONE Collaborative Research Group. JAMA. 1998;279:839-46.

189. Global BMIMC, Di Angelantonio E, Bhupathiraju ShN, Wormser D, Gao P, Kaptoge S, et al. Body-mass index and all-cause mortality: individual-participant-data metaanalysis of 239 prospective studies in four continents. Lancet. 2016;388:776-86.

190. Jordan J, Yumuk V, Schlaich M, Nilsson PM, Zahorska-Markiewicz B, Grassi G, et al. Joint statement of the European Association for the Study of Obesity and the European Society of Hypertension: obesity and difficult to treat arterial hypertension.j Hypertens. 2012;30:1047-55.

191. Whelton SP, Chin A, Xin X, He J. Effect of aerobic exercise on blood pressure: a meta-analysis of randomized, controlled trials. Ann Intern Med. 2002;136:493-503.

192. Cornelissen VA, Smart NA. Exercise training for blood pressure: a systematic review and meta-analysis. JAHA. 2013;2:e4473.

193. Rossi A, Dikareva A, Bacon SL, Daskalopoulou SS. The impact of physical activity on mortality in patients with high blood pressure: a systematic review. J Hypertens. 2012;30:1277-88.

194. Groppelli A, Giorgi DM, Omboni S, Parati G, Mancia G. Persistent blood pressure increase induced by heavy smoking. JHypertens. 1992;10:495-9.

195. Vlachopoulos C, Kosmopoulou F, Panagiotakos D, Ioakeimidis N, Alexopoulos N, Pitsavos C, et al. Smoking and caffeine have a synergistic detrimental effect on aortic stiffness and wave reflections. J Am Coll Cardiol. 2004;44:1911-7.

196. Primatesta P, Falaschetti E, Gupta S, Marmot MG, Poulter NR. Association between smoking and blood pressure: evidence from the health survey for England. Hypertension. 2001;37:187-93.

197. Ettehad D, Emdin CA, Kiran A, Anderson SG, Callender T, Emberson J, et al. Blood pressure lowering for prevention of cardiovascular disease and death: a systematic review and meta-analysis. Lancet. 2016;387:957-67.

198. Thomopoulos C, Parati G, Zanchetti A. Effects of blood pressure lowering on outcome incidence in hypertension: 4. Effects of various classes of antihypertensive drugs-overview and meta-analyses. J Hypertens. 2015;33:195-211.
199. Thomopoulos C, ParatiG,ZanchettiA. Effects of blood pressure-lowering on outcome incidence in hypertension: 5 . Head-to-head comparisons of various classes of antihypertensive drugs-overview and meta-analyses. J Hypertens. 2015;33:1321-41.

200. Williams B, Lacy PS, Thom SM, Cruickshank K, Stanton A, Collier D, et al. Differential impact of blood pressure-lowering drugs on central aortic pressure and clinical outcomes: principal results of the Conduit Artery Function Evaluation (CAFE) study. Circulation. 2006;113:1213-25.

201. Corrao G, Zambon A, Parodi A, Poluzzi E, Baldi I, Merlino L, et al. Discontinuation of and changes in drug therapy for hypertension among newly-treated patients: a populationbased study in Italy. J Hypertens. 2008;26:819-24.

202. Thomopoulos C, Parati G, Zanchetti A. Effects of bloodpressure-lowering treatment in hypertension: 9. Discontinuations for adverse events attributed to different classes of antihypertensive drugs: meta-analyses of randomized trials. JHypertens. 2016;34:1921-32.

203. Kronish IM, Woodward M, Sergie Z, Ogedegbe G, Falzon L, Mann DM. Meta-analysis: impact of drug class on adherence to antihypertensives. Circulation. 2011;123:1611-21.

204. Rasmussen ER, Pottegard A, Bygum A, von Buchwald C, Homoe P, Hallas J. Angiotensin II receptor blockers are safe in patients with prior angioedema related to angiotensin-converting enzyme inhibitors-a nationwide registrybased cohort study. J Intern Med. 2019;285:553-61.

205. Thomopoulos C, Parati G, Zanchetti A. Effects of bloodpressure-lowering treatment on outcome incidence. 12. Effects in individuals with high-normal and normal blood pressure: overview and meta-analyses of randomized trials. jHypertens. 2017;35:2150-60.

206. Ponikowski P, Voors AA, Anker SD, Bueno H, Cleland JGF, Coats AJS, et al. 2016 ESC Guidelines for the diagnosis and treatment of acute and chronic heart failure: the Task Force for the diagnosis and treatment of acute and chronic heart failure of the European Society of Cardiology (ESC)Developed with the special contribution of the Heart Failure Association (HFA) of the ESC. Eur Heart J. 2016;37:2129-200.

207. ALLHAT Officers and Coordinators for the ALLHAT Collaborative Research Group. The Antihypertensive and LipidLowering Treatment to Prevent Heart Attack Trial. Major outcomes in high-riskhypertensive patients randomized to angiotensin-converting enzyme inhibitor or calcium channel blocker vs diuretic: the Antihypertensive and LipidLowering Treatment to Prevent Heart Attack Trial (ALLHAT). JAMA. 2002;288:2981-97.

208. Wright JT Jr., Dunn JK, Cutler JA, Davis BR, Cushman WC Ford CE, et al. Outcomes in hypertensive black and nonblackpatients treated with chlorthalidone, amlodipine, and lisinopril.JAMA. 2005;293:1595-608.

209. Wright JT Jr., Harris-Haywood S, Pressel S, Barzilay J, Baimbridge $\mathrm{C}$, Bareis $\mathrm{CJ}$, et al. Clinical outcomes by race in hypertensive patients with and without the metabolic syndrome: Antihypertensive and Lipid-Lowering Treatment to Prevent Heart Attack Trial (ALLHAT). Arch Intern Med. 2008;168:207-17.

210. Fried LF, Emanuele N, Zhang JH, Brophy M, Conner TA, Duckworth W, et al. Combined angiotensin inhibition for the treatment of diabetic nephropathy. N Engl J Med. 2013;369:1892-903.

211. ONTARGET Investigators, Yusuf S, Teo KK, Pogue J, Dyal L, Copland I, et al. Telmisartan, ramipril, or both in patients at high risk for vascular events. N Engl J Med. 2008;358:1547-59. 
212. Parving HH, Brenner BM, McMurray JJ, de Zeeuw D, HaffnerSM, SolomonSD, etal.Cardiorenalend pointsin a trial of aliskirenfor type2 diabetes. NEnglJMed.2012;367:2204-13.

213. Engberink ORH, Frenkel WJ, van den Bogaard B, Brewster LM, Vogt L, van den Born BJ. Effects of thiazide-type and thiazide-like diuretics on cardiovascular events and mortality: systematic review and meta-analysis. Hypertension. 2015;65:1033-40.

214. Roush GC, Ernst ME, Kostis JB, Tandon S, Sica DA. Headto-head comparisons of hydrochlorothiazide with indapamide and chlorthalidone: antihypertensive and metabolic effects. Hypertension. 2015;65:1041-6.

215. Zillich AJ, Garg J, Basu S, Bakris GL, Carter BL. Thiazide diuretics, potassium, and the development of diabetes: a quantitative review. Hypertension. 2006;48:219-24.

216. Brown MJ, Williams B, Morant SV, Webb DJ, Caulfield MJ, Cruickshank JK, et al. Effect of amiloride, or amiloride plus hydrochlorothiazide, versus hydrochlorothiazide on glucose tolerance and blood pressure (PATHWAY-3): a parallel-group, double-blind randomised phase 4 trial. Lancet Diabetes Endocrinol.2016;4:136-47.

217. Pedersen SA, Gaist D, Schmidt SAJ, Holmich LR, Friis S, Pottegard A. Hydrochlorothiazide use and risk of nonmelanoma skin cancer: a nationwide case-control study from Denmark. JAm Acad Dermatol. 2018;78:673-681e9.

218. Pottegard A, Hallas J, Olesen M, Svendsen MT, Habel LA, Friedman GD, et al. Hydrochlorothiazide use is strongly associated with risk of lip cancer. J Intern Med. 2017;282:322-31.

219. Law MR, Morris JK, Wald NJ. Use of blood pressure lowering drugs in the prevention of cardiovascular disease: meta-analysis of 147 randomised trials in the context of expectations from prospective epidemiological studies. BMJ. 2009;338:b1665.

220. Paravastu SC, Mendonca DA, Da Silva A. Beta blockers for peripheral arterial disease. Cochrane Database Syst Rev. 2013; https://doi.org/10.1002/14651858.CD005508.pub3.

221. Radack K, Deck C. Beta-adrenergic blocker therapy does not worsen intermittent claudication in subjects with peripheral arterial disease. A meta-analysis of randomized controlled trials. Arch Intern Med. 1991;151:1769-76.

222. Wang WH, Cheng CC, Mar GY, Wei KC, Huang WC, Liu CP. Improving outcomes in chronic obstructive pulmonary disease by taking beta-blockers after acute myocardial infarction: a nationwide observational study. Heart Vessels. 2019;34:1158-67.

223. Bakris GL, Fonseca V, Katholi RE, McGill JB, Messerli FH, Phillips RA, et al. Metabolic effects of carvedilol vs metoprolol in patients with type 2 diabetes mellitus and hypertension: a randomized controlled trial. JAMA. 2004;292:2227-36.

224. Ayers K, Byrne LM, DeMatteo A, Brown NJ. Differential effects of nebivolol and metoprolol on insulin sensitivity and plasminogen activator inhibitor in the metabolic syndrome. Hypertension. 2012;59:893-8.

225. Williams B, MacDonald TM, Morant S, Webb DJ, Sever P, McInnes G, etal.Spironolactoneversus placebo, bisoprolol, and doxazosin to determine the optimal treatment for drug-resistant hypertension (PATHWAY-2): a randomised, double-blind, crossover trial. Lancet. 2015;386:2059-68.

226. ALLHAT Collaborative Research Group. Major cardiovascular events in hypertensive patients randomized to doxazosin vs chlorthalidone: the antihypertensive and lipidlowering treatment to prevent heart attack trial (ALLHAT). JAMA. 2000;283:1967-75.

227. Law MR, Wald NJ, Morris JK, Jordan RE. Value of low dose combination treatment with blood pressure lo- wering drugs: analysis of 354 randomised trials. BMJ. 2003;326:1427.

228. WaldDS, LawM, Morris JK, BestwickJP, Wald NJ.Combination therapyversus monotherapyin reducingblood pressure: meta-analysis on 11,000 participants from 42 trials. Am J Med.2009;122:290-300.

229. GuptaAK, Arshad S, Poulter NR. Compliance, safety, and effectiveness of fixed-dose combinations of antihypertensive agents: a meta-analysis. Hypertension. 2010;55:399-407.

230. MacDonald TM, Williams B, Webb DJ, Morant S, Caulfield M, CruickshankJK, et al. Combination therapy is superior to sequential Monotherapy for the initial treatment of hypertension: a double-blind randomized controlled trial. JAHA. 2017; https://doi.org/10.1161/JAHA.117.006986.

231. Corrao G, Parodi A, Zambon A, Heiman F, Filippi A, Cricelli $\mathrm{C}$, et al. Reduced discontinuation of antihypertensive treatment by two-drug combination as first step. Evidence from daily life practice. JHypertens. 2010;28:1584-90.

232. Dahlof B, Sever PS, Poulter NR, Wedel H, Beevers DG, Caulfield M, et al. Prevention of cardiovascular events with an antihypertensive regimen of amlodipine adding perindopril as required versus atenolol adding bendroflumethiazide as required, in the Anglo-Scandinavian Cardiac Outcomes Trial-Blood Pressure Lowering Arm (ASCOTBPLA): a multicentre randomised controlled trial. Lancet. 2005;366:895-906.

233. MatsuzakiM,OgiharaT,UmemotoS, RakugiH, MatsuokaH, Shimada K, et al. Prevention of cardiovascular events with calcium channel blocker-based combination therapies in patients with hypertension: a randomized controlled trial. JHypertens. 2011;29:1649-59.

234. Jamerson K, Weber MA, Bakris GL, Dahlof B, Pitt B, Shi V, et al. Benazepril plus amlodipine or hydrochlorothiazide for hypertension in high-risk patients. N Engl J Med. 2008;359:2417-28.

235. Volpe M, Rump CL, Ammentorp B, Laeis P. Efficacy and safety of triple antihypertensive therapy with the olmesartan/ amlodipine/hydrochlorothiazide combination. Clin Drug Investig. 2012;32:649-64.

236. Ference BA, Julius S, Mahajan N, Levy PD, Williams KA Sr., Flack JM. Clinical effect of naturally random allocation to lower systolic blood pressure beginning before the development of hypertension. Hypertension. 2014;63:1182-8.

237. Thomopoulos C, Parati G, Zanchetti A. Effects of blood pressure lowering on outcome incidence in hypertension: 7. Effects of more vs. less intensive blood pressure lowering and different achieved blood pressure levels-updated overview and meta-analyses of randomized trials. J Hypertens. 2016;34:613-22.

238. Thomopoulos C, Parati G, Zanchetti A. Effects of blood pressure lowering on outcome incidence in hypertension: 2. Effects at different baseline and achieved blood pressure levels-overview and meta-analyses of randomized trials. j Hypertens. 2014;32:2296-304.

239. Thomopoulos C, Parati G, Zanchetti A. Effects of blood pressure-lowering treatment on cardiovascular outcomes and mortality: 13-benefits and adverse events in older and younger patients with hypertension: overview, meta-analyses and meta-regression analyses of randomized trials. JHypertens. 2018;36:1622-36.

240. Brunstrom M, Carlberg B. Association of blood pressure lowering with mortality and cardiovascular disease across blood pressure levels: a systematic review and meta-analysis. JAMAIntern Med. 2018;178:28-36.

241. Beckett NS, Peters R, Fletcher AE, Staessen JA, Liu L, Dumitrascu D, et al. Treatment of hypertension in patients 80 years of age or older. NEngl J Med. 2008;358:1887-98. 
242. Xie X, Atkins E, Lv J, Bennett A, Neal B, Ninomiya T, et al. Effects of intensiveblood pressurelowering on cardiovascular and renal outcomes: updated systematic review and metaanalysis. Lancet. 2016;387:435-43.

243. Thomopoulos C, Parati G, Zanchetti A. Effects of blood pressure lowering treatment in hypertension: 8. Outcome reductions vs. discontinuations because of adverse drug events-meta-analyses of randomized trials. J Hypertens. 2016;34:1451-63.

244. Wright JT Jr., Whelton PK, Reboussin DM. A randomized trial of intensive versus standard blood-pressure control. NEngl J Med. 2016;374:2294.

245. Myers MG. A short history of automated office blood pressure-15 years to SPRINT. J Clin Hypertens. 2016;18:721-4.

246. Franklin SS, Gustin WT, Wong ND, Larson MG, Weber MA, Kannel WB, et al. Hemodynamic patterns of age-related changes in blood pressure. The Framingham Heart Study. Circulation. 1997;96:308-15.

247. Mancia G, Parati G, Bilo G, Gao P, Fagard R, Redon J, et al. Ambulatory blood pressure values in the ongoing telmisartan alone and in combination with ramipril global endpoint trial (ONTARGET). Hypertension. 2012;60:1400-6.

248. Duncker DJ, Bache RJ. Regulation of coronary blood flow during exercise. Physiol Rev. 2008;88:1009-86.

249. Sanders M, White FC, Peterson TM, Bloor CM. Characteristics of coronary blood flow and transmural distribution in miniature pigs. Am J Physiol. 1978;235:H601-9.

250. O'Rourke MF, Hashimoto J. Mechanical factors in arterial aging: a clinical perspective.JAm Coll Cardiol.2007;50:1-13.

251. Leung MC, Meredith IT, Cameron JD. Aortic stiffness affects the coronary blood flow response to percutaneous coronary intervention. Am J Physiol Heart Circ Physiol. 2006;290:H624-H30.

252. Saito M, Okayama H, Nishimura K, Ogimoto A, Ohtsuka T, Inoue $\mathrm{K}$, et al. Possible link between large artery stiffness and coronary flow velocity reserve. Heart. 2008;94:e20.

253. Kingwell BA, Waddell TK, MedleyTL, Cameron JD, Dart AM. Large artery stiffness predicts ischemic threshold in patients with coronary artery disease. J Am Coll Cardiol. 2002;40:773-9.

254. Weber T, Lang I, Zweiker R, Horn S, Wenzel RR, Watschinger B, et al. Hypertension and coronary artery disease: epidemiology, physiology, effects of treatment, and recommendations : A joint scientific statement from the Austrian Society of Cardiology and the Austrian Society of Hypertension. Wien Klin Wochenschr. 2016;128:467-79.

255. Nissen SE, Tuzcu EM, Libby P, Thompson PD, Ghali M, Garza D, et al. Effect of antihypertensive agents on cardiovascular events in patients with coronary disease and normal blood pressure: the CAMELOT study: a randomized controlled trial. JAMA. 2004;292:2217-25.

256. Messerli FH, Panjrath GS. The J-curve between blood pressure and coronary artery disease or essential hypertension: exactly how essential? JAm Coll Cardiol. 2009;54:1827-34.

257. Auer J, Sharman JE, Weber T. J-curves in hypertension: what do they tell us about treatment of high blood pressure? Eur Heart J. 2018;39:3115-8.

258. Beddhu S, Chertow GM, Cheung AK, Cushman WC, Rahman M, Greene T, et al. Influence of Baseline Diastolic Blood Pressure on Effects of Intensive Compared With Standard Blood Pressure Control. Circulation. 2018;137:134-43.

259. Thomopoulos C, Parati G, Zanchetti A. Effects of bloodpressure-lowering treatment on outcome incidence in hypertension. 11. Effects of total cardiovascular risk and achieved blood pressure: overview and meta-analyses of randomized trials. jHypertens. 2017;35:2138-49.
260. Dagenais GR, Pogue J, Fox K, Simoons ML, Yusuf S. Angiotensin-converting-enzyme inhibitors in stable vascular disease without left ventricular systolic dysfunction or heart failure: a combined analysis of three trials. Lancet. 2006;368:581-8.

261. HeartOutcomes PreventionEvaluation Study Investigators, Yusuf S, Sleight P, Pogue J, Bosch J, Davies R, et al. Effects of an angiotensin-converting-enzyme inhibitor, ramipril, on cardiovascular events in high-risk patients. N Engl J Med. 2000;342:145-53.

262. Task Force Members, Montalescot G, Sechtem U, Achenbach S, Andreotti F, Arden C, et al. 2013 ESC guidelines on the management of stable coronary artery disease: the Task Force on the management of stable coronary artery disease of the European Society of Cardiology. Eur Heart J. 2013;34:2949-3003.

263. Rosendorff C, Lackland DT, Allison M, Aronow WS, Black HR, Blumenthal RS, et al. Treatment of hypertension in patients with coronary artery disease: a scientific statement from the American Heart Association, American College of Cardiology, and American Society of Hypertension. Hypertension. 2015;65:1372-407.

264. Bozkurt B, Aguilar D, Deswal A, Dunbar SB, Francis GS, Horwich T, et al. Contributory risk and management of Comorbidities of hypertension, obesity, diabetes mellitus, hyperlipidemia, and metabolic syndrome in chronic heart failure: a scientific statement from the American heart association. Circulation. 2016;134:e535-e78.

265. Chae CU, Pfeffer MA, Glynn RJ, Mitchell GF, Taylor JO, Hennekens $\mathrm{CH}$. Increased pulse pressure and risk of heart failure in the elderly. JAMA. 1999;281:634-9.

266. Weber T, Chirinos JA. Pulsatile arterial haemodynamics in heartfailure. Eur Heart J. 2018;39:3847-54.

267. Adams KF Jr., Fonarow GC, Emerman CL, LeJemtel TH, Costanzo MR, Abraham WT, et al. Characteristics and outcomes of patients hospitalized for heart failure in the United States: rationale, design, and preliminary observations from the first 100,000 cases in the Acute Decompensated Heart Failure National Registry (ADHERE). Am Heart J. 2005;149:209-16.

268. Kato S, Onishi K, Yamanaka T, Takamura T, Dohi K, Yamada N, et al. Exaggerated hypertensive response to exercise in patients with diastolic heart failure. Hypertension. 2008;31:679-84.

269. Devereux RB, Wachtell K, Gerdts E, Boman K, Nieminen MS, Papademetriou V, et al. Prognostic significance of left ventricular mass change during treatment of hypertension. JAMA. 2004;292:2350-6.

270. Fagard RH, Celis H, Thijs L, Wouters S. Regression of left ventricular mass by antihypertensive treatment: a metaanalysis of randomized comparativestudies. Hypertension. 2009;54:1084-91.

271. Verdecchia P, Staessen JA, Angeli F, de Simone G, Achilli A, Ganau A, et al. Usual versus tight control of systolic blood pressure in non-diabetic patients with hypertension (Cardio-Sis): an open-label randomised trial. Lancet. 2009;374:525-33.

272. Drazner MH, Rame JE, Marino EK, Gottdiener JS, Kitzman DW, Gardin JM, et al. Increased left ventricular mass is a risk factor for the development of a depressed left ventricular ejection fraction within five years: the Cardiovascular Health Study. JAm Coll Cardiol. 2004;43:2207-15.

273. Kostis JB, Davis BR, Cutler J, Grimm RH Jr., Berge KG, Cohen JD, et al. Prevention of heart failure by antihypertensive drug treatment in older persons with isolated systolic hypertension. SHEP Cooperative Research Group. JAMA. 1997;278:212-6. 
274. McMurray JJ, Adamopoulos S, Anker SD, Auricchio A, Bohm M, Dickstein K, et al. ESC Guidelines for the diagnosis and treatment of acute and chronic heart failure 2012: The Task Force for the Diagnosis and Treatment of Acute and Chronic Heart Failure 2012 of the European Society of Cardiology. Developed in collaboration with the Heart Failure Association (HFA) of the ESC. eur Heart J. 2012of;33:1787-847.

275. Packer M, Poole-Wilson PA, Armstrong PW, Cleland JG, Horowitz JD, Massie BM, et al. Comparative effects of low and high doses of the angiotensin-converting enzyme inhibitor, lisinopril, on morbidity and mortality in chronic heart failure. ATLAS Study Group. Circulation. 1999;100:2312-8.

276. Bristow MR, Gilbert EM, Abraham WT, Adams KF, Fowler MB, Hershberger RE, et al. Carvedilol produces doserelated improvements in left ventricular function and survival in subjects with chronic heart failure. MOCHA Investigators. Circulation. 1996;94:2807-16.

277. Anand IS, Rector TS, Kuskowski M, Thomas S, Holwerda NJ, Cohn JN. Effect of baseline and changes in systolic blood pressure over time on the effectiveness of valsartan in the Valsartan Heart Failure Trial. Circ Heart Fail. 2008;1:34-42.

278. Rouleau JL, Roecker EB, Tendera M, Mohacsi P, Krum H, Katus HA, et al. Influence of pretreatment systolic blood pressure on the effect of carvedilol in patients with severe chronic heart failure: the Carvedilol Prospective Randomized Cumulative Survival (COPERNICUS) study. J Am Coll Cardiol. 2004;43:1423-9.

279. Packer M, O'Connor CM, Ghali JK, Pressler ML, Carson PE, Belkin RN, et al. Effect of amlodipine on morbidity and mortality in severe chronic heart failure. Prospective Randomized Amlodipine Survival Evaluation Study Group. NEnglJ Med. 1996;335:1107-14.

280. Cohn JN, Ziesche S, Smith R, Anand I, Dunkman WB, Loeb $\mathrm{H}$, et al. Effect of the calcium antagonist felodipine as supplementary vasodilator therapyin patients with chronic heart failure treated with enalapril:V-HeFT III. VasodilatorHeart Failure Trial (V-HeFT) Study Group. Circulation. 1997;96:856-63.

281. Lip GYH, Coca A, Kahan T, Boriani G, Manolis AS, Olsen MH, et al. Hypertension and cardiac arrhythmias: a consensus document from the European Heart Rhythm Association (EHRA) and ESC Council on Hypertension, endorsed by the HeartRhythm Society (HRS), Asia-Pacific Heart Rhythm Society (APHRS) and Sociedad Latinoamericana deEstimulacion Cardiaca y Electrofisiologia (SOLEACE). Europace. 2017;19:891-911.

282. Haywood LJ, Ford CE, Crow RS, Davis BR, Massie BM, Einhorn PT, et al. Atrial fibrillation at baseline and during follow-up in ALLHAT (Antihypertensive and Lipid-Lowering Treatment to Prevent Heart Attack Trial). J Am Coll Cardiol. 2009;54:2023-31.

283. Thomas MC, Dublin S, Kaplan RC, Glazer NL, Lumley T, Longstreth WT Jr., et al. Blood pressure control and risk of incident atrial fibrillation. Am J Hypertens. 2008;21:1111-6.

284. Healey JS, Baranchuk A, Crystal E, Morillo CA, Garfinkle M, Yusuf S, et al. Prevention of atrial fibrillation with angiotensin-converting enzyme inhibitors and angiotensin receptor blockers: a meta-analysis. J Am Coll Cardiol. 2005;45:1832-9.

285. Kirchhof P, Benussi S, Kotecha D, Ahlsson A, Atar D, Casadei B, et al. 2016 ESC Guidelines for the management of atrial fibrillation developed in collaboration with EACTS. Eur Heart J2016;37:2893-962

286. Pisters R, Lane DA, Nieuwlaat R, de Vos CB, Crijns HJ, Lip GY. Anovel user-friendly score (HAS-BLED) to assess 1-year risk of majorbleedingin patients with atrialfibrillation: theEuro Heart Survey. Chest. 2010;138:1093-100.

287. Rao MP, Halvorsen S, Wojdyla D, Thomas L, Alexander JH, Hylek EM, et al. Blood pressure control and risk of stroke or systemic embolism in patients with atrial fibrillation: results from the apixaban for reduction in stroke and other Thromboembolic events in atrial fibrillation (ARISTOTLE) trial. JAHA. 2015; https://doi.org/10.1161/ JAHA.115.002015.

288. Levy D, Anderson KM, Savage DD, Balkus SA, Kannel WB, Castelli WP. Risk of ventricular arrhythmias in left ventricular hypertrophy: the Framingham Heart Study. Am J Cardiol. 1987;60:560-5.

289. Haider AW, Larson MG, Benjamin EJ, Levy D. Increased left ventricular mass and hypertrophy are associated with increased risk for sudden death. J Am Coll Cardiol. 1998;32:1454-9.

290. Wachtell K, Okin PM, Olsen MH, Dahlof B, Devereux RB, Ibsen $\mathrm{H}$, et al. Regression of electrocardiographic left ventricular hypertrophy during antihypertensive therapy and reduction in sudden cardiac death: the LIFE Study. Circulation. 2007;116:700-5.

291. Siscovick DS, Raghunathan TE, Psaty BM, Koepsell TD, Wicklund KG, Lin X, et al. Diuretic therapy for hypertension and the risk of primary cardiac arrest. $\mathrm{N}$ Engl J Med. 1994;330:1852-7.

292. Kang TS, ParkS. Antihypertensive treatment in severe aortic stenosis. J Cardiovasc Imaging. 2018;26:45-53.

293. Lindman BR, Otto CM. Time to treat hypertension in patients with aortic stenosis. Circulation. 2013;128:1281-3.

294. Rieck AE, Cramariuc D, Boman K, Gohlke-Barwolf C, Staal EM, Lonnebakken MT, et al. Hypertension in aortic stenosis: implications for left ventricular structure and cardiovascular events. Hypertension. 2012;60:90-7.

295. O'Brien KD, Zhao XQ, Shavelle DM, Caulfield MT, Letterer RA, Kapadia SR, et al. Hemodynamic effects of the angiotensin-converting enzyme inhibitor, ramipril, in patients with mild to moderate aortic stenosis and preserved left ventricular function. J Investig Med. 2004;52:185-91.

296. Chockalingam A, Venkatesan S, Subramaniam T, Jagannathan V, Elangovan S, Alagesan R, et al. Safety and efficacy of angiotensin-converting enzyme inhibitors in symptomatic severe aortic stenosis: Symptomatic Cardiac ObstructionPilot Study of Enalapril in Aortic Stenosis (SCOPE-AS). Am Heart J. 2004;147:E19.

297. Evangelista A, Tornos P, Sambola A, Permanyer-Miralda G, Soler-Soler J. Long-term vasodilator therapy in patients with severe aortic regurgitation. N Engl J Med. 2005;353:1342-9.

298. Su TC, Jeng JS, Chien KL, Sung FC, Hsu HC, Lee YT. Hypertension status is the major determinant of carotid atherosclerosis: a community-based study in Taiwan. Stroke. 2001;32:2265-71.

299. WangJG, StaessenJA, LiY, Van BortelLM, NawrotT, FagardR, et al. Carotid intima-media thickness and antihypertensive treatment: a meta-analysis of randomized controlled trials. Stroke. 2006;37:1933-40.

300. Zanchetti A, Crepaldi G, Bond MG, Gallus G, Veglia F, Mancia $G$, et al. Different effects of antihypertensive regimens based on fosinopril or hydrochlorothiazide with or without lipid lowering by pravastatin on progression of asymptomatic carotid atherosclerosis: principal results of PHYLLIS-a randomized double-blind trial. Stroke. 2004;35:2807-12.

301. Zanchetti A, Hennig M, Hollweck R, Bond G, Tang R, Cuspidi $\mathrm{C}$, et al. Baseline values but not treatment-induced changes in carotid intima-media thickness predict incidentcardiovascular events in treatedhypertensive patients: 
findings in the European Lacidipine Study on Atherosclerosis (ELSA). Circulation. 2009;120:1084-90.

302. Emdin CA, Anderson SG, Callender T, Conrad N, SalimiKhorshidi G, Mohseni H, et al. Usual blood pressure, peripheral arterial disease, and vascular risk: cohort study of 4.2 million adults. BMJ. 2015;351:h4865.

303. Singer DR, KiteA. Management of hypertension in peripheral arterial disease: does the choice of drugs matter? Eur J Vasc Endovasc Surg. 2008;35:701-8.

304. Ait-Oufella H, Collin C, Bozec E, Laloux B, Ong KT, Dufouil C, et al. Long-term reduction in aortic stiffness: a 5.3year follow-up in routine clinical practice. J Hypertens. 2010;28:2336-41.

305. Laurent S, Boutouyrie P, Mechanism VC. Dose-dependent arterial destiffening and inward remodeling after olmesartan in hypertensives with metabolic syndrome. Hypertension. 2014;64:709-16.

306. Ong KT, Delerme S, Pannier B, Safar ME, Benetos A, Laurent $\mathrm{S}$, et al. Aortic stiffness is reduced beyond blood pressure lowering by short-term and long-term antihypertensive treatment: a meta-analysis of individual data in 294 patients. J Hypertens. 2011;29:1034-42.

307. Shahin Y, Khan JA, Chetter I. Angiotensin converting enzyme inhibitors effect on arterial stiffness and wave reflections: a meta-analysis and meta-regression of randomised controlled trials. Atherosclerosis. 2012;221:18-33.

308. Erbel R, Aboyans V, Boileau C, Bossone E, Bartolomeo RD, Eggebrecht H, et al. 2014 ESC Guidelines on the diagnosis and treatment of aortic diseases: Document covering acute and chronic aortic diseases of the thoracic and abdominal aorta of the adult. The Task Force for the Diagnosis and Treatment of Aortic Diseases of the European Society of Cardiology (ESC). Eur Heart J. 2014;35:2873-926.

309. Groenink M, den Hartog AW, Franken R, Radonic T, de Waard V, Timmermans J, et al. Losartan reduces aortic dilatation rate in adults with Marfan syndrome: a randomized controlled trial. Eur Heart J. 2013;34:3491-500.

310. Shores J, Berger KR, Murphy EA, Pyeritz RE. Progression of aortic dilatation and the benefit of long-term betaadrenergic blockade in Marfan's syndrome. N Engl J Med. 1994;330:1335-41.

311. Teixido-Tura G, FortezaA, Rodriguez-PalomaresJ, Gonzalez Mirelis J, Gutierrez L, Sanchez V, et al. Losartan versus atenolol for prevention of aortic dilation in patients with Marfan syndrome. JAm Coll Cardiol. 2018;72:1613-8.

312. Mehta RH, O'Gara PT, Bossone E, Nienaber CA, Myrmel T, Cooper JV, et al. Acute type A aortic dissection in the elderly: clinical characteristics, management, and outcomes in the current era. J Am Coll Cardiol. 2002;40:685-92.

313. Delsart P, Ledieu GJ, Ramdane N, Sobocinski JP, Clough RE, Azzaoui RO, et al. Impact of the Management of Type B Aortic Dissection on the Long-Term Blood Pressure. Am J Cardiol.2017;120:484-8.

314. Suzuki T, IsselbacherEM, Nienaber CA, PyeritzRE, EagleKA, Tsai TT, et al. Type-selective benefits of medications in treatment of acute aortic dissection (from the International Registry of Acute Aortic Dissection [IRAD]). Am J Cardiol. 2012;109:122-7.

315. Gomes MB, Tannus LR, Matheus AS, Cobas RA, Palma CC, Silva AT, et al. Prevalence, awareness, and treatment of hypertension in patients with type 1 diabetes: a nationwide multicenter study in Brazil. Int J Hypertens. 2013;2013:565263.

316. Ferrannini E, Cushman WC. Diabetes and hypertension: the bad companions. Lancet. 2012;380:601-10.

317. Wijkman M, Lanne T, Engvall J, Lindstrom T, Ostgren CJ, Nystrom FH. Masked nocturnal hypertension-a no- vel marker of risk in type 2 diabetes. Diabetologia. 2009;52:1258-64.

318. TarnowL, Rossing P, Jensen C, Hansen BV,ParvingHH.Longterm renoprotective effect of nisoldipine and lisinopril in type 1 diabetic patients with diabetic nephropathy. Diabetes Care. 2000;23:1725-30.

319. Emdin CA, Rahimi K, Neal B, Callender T, Perkovic V, Patel A. Blood pressure lowering in type 2 diabetes: a systematic review and meta-analysis. JAMA. 2015;313:603-15.

320. Thomopoulos C, Parati G, Zanchetti A. Effects of bloodpressure-lowering treatment on outcome incidence in hypertension: 10-Should blood pressure management differ in hypertensive patients with and without diabetes mellitus? Overview and meta-analyses of randomized trials. JHypertens. 2017;35:922-44.

321. Brunstrom M, Carlberg B. Effect of antihypertensive treatment at different blood pressure levels in patients with diabetes mellitus: systematic review and meta-analyses. BMJ.2016;352:i717.

322. Patel A, ADVANCE collaborative group, MacMahon S, MacMahon S, Chalmers J, Neal B, Woodward M, et al. Effects of a fixed combination of perindopril and indapamide on macrovascular and microvascular outcomes in patients with type 2 diabetes mellitus (the ADVANCE trial): a randomised controlled trial. Lancet. 2007;370:829-40.

323. ACCORD Study Group, Cushman WC, Evans GW, Byington RP, Goff DC Jr., Grimm RH Jr., et al. Effects of intensive blood-pressure control in type 2 diabetes mellitus. N Engl J Med. 2010;362:1575-85.

324. Buckley LF, Dixon DL, Wohlford GF 4th, Wijesinghe DS, Baker WL, Van Tassell BW. Intensive versus standard blood pressure control in SPRINT-eligible participants of ACCORD-BP. Diabetes Care. 2017;40:1733-8.

325. Margolis KL, O'Connor PJ, Morgan TM, Buse JB, Cohen RM, Cushman WC, et al. Outcomes of combined cardiovascular risk factor management strategies in type 2 diabetes: the ACCORD randomized trial. Diabetes Care. 2014;37:1721-8.

326. Cooper-DeHoff RM, Gong Y, Handberg EM, Bavry AA, Denardo SJ, Bakris GL, et al. Tight blood pressure control and cardiovascular outcomes among hypertensive patients with diabetes and coronary artery disease. JAMA. 2010;304:61-8.

327. Bress AP, King JB, Kreider KE, Beddhu S, Simmons DL, Cheung AK, et al. Effect of intensive versus standard blood pressure treatment according to baseline prediabetes status: a post hoc analysis of a randomized trial. Diabetes Care 2017;40:1401-8.

328. Hansson L, Zanchetti A, Carruthers SG, Dahlof B, Elmfeldt D, Julius S, et al. Effects of intensive blood-pressure lowering and low-dose aspirin in patients with hypertension: principal results of the Hypertension Optimal Treatment (HOT) randomised trial. HOT Study Group. Lancet. 1998;351:1755-62.

329. UK Prospective Diabetes Study Group. Tight blood pressure control and risk of macrovascular and microvascular complications in type 2 diabetes: UKPDS 38. BMJ. 1998;317:703-13.

330. Wing RR, Lang W, Wadden TA, Safford M, Knowler WC, Bertoni AG, et al. Benefits of modest weight loss in improving cardiovascular risk factors in overweight and obese individuals with type 2 diabetes. Diabetes Care. 2011;34:1481-6.

331. ManciaG, GrassiG. Blood pressure targetsintype2 diabetes. Evidence against or in favour of an aggressive approach. Diabetologia. 2018;61:517-25.

332. Palmer SC, Mavridis D, Navarese E, Craig JC, Tonelli M, Salanti $\mathrm{G}$, et al. Comparative efficacy and safety of blood pres- 
sure-lowering agents in adults with diabetes and kidney disease: a networkmeta-analysis. Lancet. 2015;385:2047-56.

333. Mann JF, Schmieder RE, McQueen M, Dyal L, Schumacher H, Pogue J, et al. Renal outcomes with telmisartan, ramipril, or both, in people at high vascular risk (the ONTARGET study): a multicentre, randomised, double-blind, controlled trial. Lancet. 2008;372:547-53.

334. Angeli F, Reboldi G, Mazzotta G, Poltronieri C, Garofoli M, Ramundo E, et al. Safety and efficacy of aliskiren in the treatment of hypertension and associated clinical conditions. CDS. 2012;7:76-85.

335. Qayyum R, Adomaityte J. A meta-analysis of the effect of thiazolidinediones on blood pressure. J Clin Hypertens. 2006;8:19-28

336. Wang B, Zhong J, Lin $\mathrm{H}$, Zhao Z, Yan Z, He H, et al. Blood pressure-lowering effects of GLP-1 receptor agonists exenatide and liraglutide: a meta-analysis of clinical trials. Diabetes Obes Metab. 2013;15:737-49.

337. Baker WL, Buckley LF, Kelly MS, Bucheit JD, Parod ED, Brown R, et al. Effects of sodium-glucose cotransporter 2 inhibitors on 24-hour ambulatory blood pressure: a systematic review and meta-analysis. J Am Heart Assoc. 2017; https://doi.org/10.1161/JAHA.117.005686.

338. Mancia G, Cannon CP, Tikkanen I, Zeller C, Ley L, Woerle HJ, et al. Impact of empagliflozin on blood pressure in patients with type 2 diabetes mellitus and hypertension by background antihypertensive medication. Hypertension. 2016;68:1355-64.

339. Zinman B, Wanner C, Lachin JM, Fitchett D, Bluhmki E, HantelS, etal.Empagliflozin, cardiovascular outcomes, and mortalityin type 2 diabetes. NEnglJMed.2015;373:2117-28.

340. Neal B, Perkovic V, Mahaffey KW, de Zeeuw D, Fulcher G, Erondu N, et al. Canagliflozin and cardiovascular and renal events in type 2 diabetes. NEnglJ Med. 2017;377:644-57.

341. Wiviott SD, Raz I, Bonaca MP, Mosenzon O, Kato ET, Cahn A, et al. Dapagliflozin and Cardiovascular Outcomes in Type 2 Diabetes. NEngl J Med. 2019;380:347-57.

342. Gerstein HC, Colhoun HM, Dagenais GR, Diaz R, Lakshmanan M, Pais P, et al. Dulaglutide and cardiovascular outcomes in type 2 diabetes (REWIND): a double-blind, randomised placebo-controlled trial. J Am Heart Assoc. 2018;7(8):pii:e008994. https://doi.org/10.1161/JAHA.118. 008994

343. Marso SP, Bain SC, Consoli A, Eliaschewitz FG, Jodar E, Leiter LA, et al. Semaglutide and cardiovascular outcomes in patients with type 2 diabetes. N Engl J Med. 2016;375:1834-44.

344. Marso SP, Daniels GH, Brown-Frandsen K, Kristensen P, Mann JF, Nauck MA, et al. Liraglutide and cardiovascular outcomes in type 2 diabetes. NEnglJ Med. 2016;375:311-22.

345. Kramar R. ÖDTR (Österreichisches Dialyse- und Transplantationsregister) Jahresbericht 2017. https://www. nephrologie.at/oegn-berichte/. Zugriff zuletzt am 31.10.2019.

346. Prischl FC, Auinger M, Saemann M, Mayer G, Rosenkranz AR, Wallner $M$, et al. Diabetes-related end-stage renal disease in Austria 1965-2013. Nephrol Dial Transplant. 2015;30:1920-7.

347. Clase CM, Barzilay J, Gao P, Smyth A, Schmieder RE, Tobe $S$, et al. Acute change in glomerular filtration rate with inhibition of the renin-angiotensin system does not predict subsequent renal and cardiovascular outcomes. Kidney Int. 2017;91:683-90.

348. Schmidt M, Mansfield KE, Bhaskaran K, Nitsch D, Sorensen HT, Smeeth L, et al. Serum creatinine elevation after renin-angiotensin system blockade and long term cardiorenal risks: cohort study. BMJ.2017;356:j791.
349. Levin A, Stevens PE. Summary of KDIGO 2012 CKD guideline: Behind the scenes, need for guidance, and a framework for moving forward. Kidney Int 2014;85:49-61.

350. Thomas G, Drawz PE. BP measurement techniques: what they mean for patients with kidney disease. Clin J Am Soc Nephrol.2018;13:1124-31.

351. Drawz PE, Ix JH. BP measurement in clinical practice: time to SPRINT to guideline-recommended protocols. J Am Soc Nephrol.2018;29:383-8.

352. Minutolo R, Agarwal R, Borrelli S, Chiodini P, Bellizzi V, Nappi F, et al. Prognostic role of ambulatory blood pressure measurement in patients with nondialysis chronic kidney disease. Arch Intern Med. 2011;171:1090-8.

353. Andreadis EA, Geladari CV, Angelopoulos ET, Savva FS, Georgantoni AI, Papademetriou V. Attended and unattended automated officeblood pressuremeasurements havebetter agreement with ambulatory monitoring than conventional office readings. J Am Heart Assoc 2018;7(8):pii:e008994. https://doi.org/10.1161/JAHA.118.008994.

354. Minutolo R, De Nicola L, Gallo C, Chiodini P, Provenzano $\mathrm{M}$, Conte G, et al. Generalizability of SPRINT-CKD cohort to CKD patients referred to renal clinics. J Nephrol. 2019;32:429-35.

355. Buerkert J, Martin DR, Trigg D, Simon EE. Sodium handling by deep nephrons and the terminal collecting duct in glomerulonephritis. Kidney Int. 1991;39:850-7.

356. Buckalew VM Jr., Berg RL, Wang SR, Porush JG, Rauch S, Schulman G. Prevalence of hypertension in 1,795 subjects with chronic renal disease: the modification of diet in renal disease study baseline cohort. Modification of Diet in Renal Disease Study Group. Am J Kidney Dis. 1996;28:811-21.

357. Neumann J, Ligtenberg G, Klein II, Koomans HA, Blankestijn PJ. Sympathetic hyperactivity in chronic kidney disease: pathogenesis, clinical relevance, and treatment. Kidney Int. 2004;65:1568-76.

358. Raine AE, Bedford L, Simpson AW, Ashley CC, Brown R, Woodhead JS, et al. Hyperparathyroidism, platelet intracellular free calcium and hypertension in chronic renal failure. Kidney Int. 1993;43:700-5.

359. Passauer J, Pistrosch F, Bussemaker E, Lassig G, Herbrig K, Gross P. Reduced agonist-induced endothelium-dependentvasodilation in uremia is attributable to an impairment of vascular nitric oxide. J Am Soc Nephrol. 2005;16:959-65.

360. Yamagata K, Makino H, Iseki K, Ito S, Kimura K, Kusano E, et al. Effect of behavior modification on outcome in early- to moderate-stage chronic kidney disease: a clusterrandomized trial. Plos One. 2016;11:e151422.

361. Floege J, Barbour SJ, Cattran DC, Hogan JJ, Nachman PH, Tang SCW, et al. Management and treatment of glomerular diseases (part 1): conclusions from a Kidney Disease: Improving Global Outcomes (KDIGO) Controversies Conference. Kidney Int. 2019;95:268-80.

362. He FJ, MacGregor GA. Effect of modest salt reduction on blood pressure: a meta-analysis of randomized trials. Implications for public health. JHum Hypertens. 2002;16:761-70.

363. Vogt L, Waanders F, Boomsma F, de Zeeuw D, Navis G. Effects of dietary sodium and hydrochlorothiazide on the antiproteinuric efficacy of losartan. J Am Soc Nephrol. 2008;19:999-1007.

364. Lambers Heerspink HJ, Holtkamp FA, Parving HH, Navis GJ, Lewis JB, Ritz E, et al. Moderation of dietary sodium potentiates the renal and cardiovascular protective effects of angiotensin receptor blockers. Kidney Int. 2012;82:330-7.

365. Nerbass FB, Calice-Silva V, Pecoits-Filho R. Sodium intake and blood pressure in patients with chronic kidney disease: a salty relationship. Blood Purif. 2018;45:166-72. 
366. Eren OC, OrtizA, Afsar B, CovicA, Kuwabara M, Lanaspa MA, et al. Multilayered interplaybetween fructose and salt in development of hypertension. Hypertension. 2019;73:265-72.

367. Sinha AD, Agarwal R. Clinical pharmacology of antihypertensive therapy for the treatment of hypertension in CKD. Clin JAm Soc Nephrol. 2019;14:757-64.

368. Kent DM, Jafar TH, Hayward RA, Tighiouart H, Landa M, de Jong $\mathrm{P}$, et al. Progression risk, urinary protein excretion, and treatment effects of angiotensin-converting enzyme inhibitors in nondiabetic kidney disease. J Am Soc Nephrol. 2007;18:1959-65.

369. Brenner BM, Cooper ME, de Zeeuw D, Keane WF, MitchWE, Parving $\mathrm{HH}$, et al. Effects of losartan on renal and cardiovascular outcomes in patients with type 2 diabetes and nephropathy. NEngl J Med.2001;345:861-9.

370. LewisEJ, HunsickerLG, ClarkeWR, BerlT, Pohl MA, LewisJB, et al. Renoprotective effect of the angiotensin-receptor antagonist irbesartan in patients with nephropathy due to type 2 diabetes. NEngl J Med. 2001;345:851-60.

371. Agarwal R, Lewis R, Davis JL, Becker B. Lisinopril therapy for hemodialysis hypertension: hemodynamic and endocrine responses. Am J Kidney Dis. 2001;38:1245-50.

372. Wright JT Jr., Bakris G, Greene T, Agodoa LY, Appel LJ, Charleston J, et al. Effect of blood pressure lowering and antihypertensive drug class on progression of hypertensive kidney disease: results from the AASK trial. JAMA. 2002;288:2421-31.

373. Tepel M, Hopfenmueller W, Scholze A, Maier A, Zidek W. Effect of amlodipine on cardiovascular events in hypertensive haemodialysis patients. Nephrol Dial Transplant. 2008;23:3605-12.

374. Khosla UM, Johnson RJ. Hypertension in the hemodialysis patient and the "lag phenomenon": insights into pathophysiology and clinical management. Am J Kidney Dis. 2004;43:739-51.

375. Sinha AD, Agarwal R. Thiazides in advanced chronic kidney disease: time for a randomized controlled trial. Curr Opin Cardiol.2015;30:366-72.

376. KidneyDisease:ImprovingGlobalOutcome (KDIGO) Blood Pressure Working Group. KDIGO clinical practice guideline for the management of blood pressure in chronic kidney disease. KidneyInt2012;2(Suppl):337-414.

377. Chlorthalidone in Chronic Kidney Disease (CLICK). Accessibleat:https://clinicaltrials.gov/ct2/show/NCT02841280. Last accessed October 31,2019.

378. Liang W, Ma H, Cao L, Yan W, Yang J. Comparison of thiazide-like diuretics versus thiazide-type diuretics: a metaanalysis. J Cell Mol Med. 2017;21:2634-42.

379. Pareek AK, Messerli FH, Chandurkar NB, Dharmadhikari SK, Godbole AV, Kshirsagar PP, et al. Efficacy of lowdose chlorthalidone and hydrochlorothiazide as assessed by 24 -h ambulatory blood pressure monitoring. J Am Coll Cardiol. 2016:67:379-89.

380. Wright JT Jr., Probstfield JL, Cushman WC, Pressel SL, Cutler JA, Davis BR, et al. ALLHAT findings revisited in the context of subsequent analyses, other trials, and metaanalyses. Arch Intern Med. 2009;169:832-42.

381. Moes AD, Hesselink DA, van den Meiracker AH, Zietse R, Hoorn EJ. Chlorthalidone versus amlodipine for hypertension in kidney transplant recipients treated with tacrolimus: a randomized crossover trial. Am J Kidney Dis. 2017;69:796-804.

382. Lepage L, Dufour AC, Doiron J, Handfield K, Desforges K, Bell R, et al. Randomized clinical trial of sodium polystyrene sulfonate for the treatment of mild hyperkalemia in CKD. Clin JAm Soc Nephrol. 2015;10:2136-42.
383. Kloner RA, Gross C, Yuan J, Conrad A, Pergola PE. Effect of patiromer in hyperkalemic patients taking and not taking RAAS inhibitors. J Cardiovasc Pharmacol Ther. 2018;23:524-31.

384. Evans M, Palaka E, Furuland H, Bennett H, Linde C, Qin L, et al. The value of maintaining normokalaemia and enabling RAASi therapy in chronic kidney disease. BMCNephrol. 2019;20:31.

385. Harel Z, Harel S, Shah PS, Wald R, Perl J, Bell CM. Gastrointestinal adverse events with sodium polystyrene sulfonate (Kayexalate) use: a systematic review. Am J Med. 2013;126:264e9-264e24.

386. Spinowitz BS, Fishbane S, Pergola PE, Roger SD, Lerma EV, Butler J, et al. Sodium zirconium cyclosilicate among individuals with hyperkalemia: a 12-month phase 3 study. Clin J Am Soc Nephrol. 2019;14:798-809.

387. Pitt B, Bushinsky DA, Kitzman DW, Ruschitzka F, Metra M, Filippatos G, et al. Evaluation of an individualized dose titration regimen of patiromer to prevent hyperkalaemia in patients with heart failure and chronic kidney disease. ESC Heart Fail. 2018;5:257-66.

388. Pitt B, Bakris GL, Weir MR, Freeman MW, Lainscak M, Mayo MR, et al. Long-term effects of patiromer for hyperkalaemia treatmentin patients with mild heartfailure and diabetic nephropathy on angiotensin-converting enzymes/ angiotensin receptor blockers: results from AMETHYSTDN.ESCHeartFail.2018;5:592-602.

389. Rosano GMC, Tamargo J, Kjeldsen KP, Lainscak M, Agewall S, Anker SD, et al. Expert consensus document on the management of hyperkalaemia in patients with cardiovascular disease treated with renin angiotensin aldosterone system inhibitors: coordinated by the Working Group on Cardiovascular Pharmacotherapy of the European Society of Cardiology. Eur Heart J Cardiovasc Pharmacother. 2018;4:180-8.

390. Schlaich MP, Socratous F, HennebryS, Eikelis N, LambertEA, Straznicky N, et al. Sympathetic activation in chronic renal failure. JAm Soc Nephrol. 2009;20:933-9.

391. Toto RD. Treatment of hypertension in chronic kidney disease. Semin Nephrol. 2005;25:435-9.

392. Man in't Veld AJ, Van den Meiracker AH, Schalekamp MA. Do beta-blockers really increase peripheral vascular resistance? Review of literature and new observations under basal conditions. Am J Hypertens 1988;1:91-6.

393. Converse RLJr., Jacobsen TN, Toto RD, Jost CM, Cosentino F, Fouad-Tarazi F, et al. Sympathetic overactivity in patients with chronic renal failure. NEngl J Med. 1992;327:1912-8.

394. Yasuda G, Saka S, Ando D, Hirawa N. Effects of doxazosin as the third agent on morning hypertension and positionrelated blood pressure changes in diabetic patients with chronic kidney disease. Clin Exp Hypertens. 2015;37:75-81.

395. Mundt HM, Matenaer M, Lammert A, Gottmann U, Kramer BK, Birck R, et al. Minoxidil for treatment of resistant hypertension in chronic kidney disease-a retrospective cohort analysis. JClin Hypertens. 2016;18:1162-7.

396. Lv J, Ehteshami P, Sarnak MJ, Tighiouart H, Jun M, Ninomiya T, etal. Effects of intensive blood pressurelowering on the progression of chronic kidney disease: a systematic review and meta-analysis. CMAJ.2013;185:949-57.

397. Malhotra R, Nguyen HA, Benavente O, Mete M, Howard BV, Mant J, et al. Association between more intensive vs less intensive blood pressure lowering and risk of mortality in chronic kidney disease stages 3 to 5: a systematic review and meta-analysis. JAMAIntern Med. 2017;177:1498-505.

398. Weir MR, Lakkis JI, Jaar B, Rocco MV, Choi MJ, Kramer HJ, et al. Use of renin-angiotensin system blockade in advanced 
CKD: an NKF-KDOQI controversies report.AmJ Kidney Dis. 2018;72:873-84.

399. Ku E, Lee BJ, Wei J, Weir MR. Hypertension in CKD: core curriculum 2019. Am J Kidney Dis. 2019;74:120-31.

400. Ku E, McCulloch CE, VittinghoffE, Lin F, Johansen KL. Use of antihypertensiveagentsand association with riskofadverse outcomes in chronic kidney disease: focus on angiotensinconverting enzyme inhibitors and Angiotensin receptor blockers. JAm Heart Assoc. 2018;7:e9992.

401. Cai J, Huang X, Zheng Z, Lin Q, Peng M, Shen D. Comparative efficacy of individual renin-angiotensin system inhibitors on major renal outcomes in diabetic kidney disease: a network meta-analysis. Nephrol Dial Transplant. 2018;33:1968-76.

402. Chang AR, Loser M, Malhotra R, Appel LJ. Blood pressure goals in patients with CKD: a review of evidence and guidelines. Clin JAm Soc Nephrol.2019;14:161-9.

403. Cheung AK, Rahman M, Reboussin DM, Craven TE, GreeneT, Kimmel PL, et al. Effects of intensive BP control in CKD. JAm Soc Nephrol.2017;28:2812-23.

404. Sim JJ, Shi J, Kovesdy CP, Kalantar-Zadeh K, Jacobsen SJ. Impact of achieved blood pressures on mortality risk and endstage renal disease among a large, diverse hypertension population. JAm Coll Cardiol. 2014;64:588-97.

405. Sim JJ, BhandariSK, ShiJ, ReynoldsK, CalhounDA, KalantarZadehK, etal.Comparativeriskofrenal, cardiovascular, and mortality outcomes in controlled, uncontrolled resistant, andnonresistanthypertension. KidneyInt. 2015;88:622-32.

406. Jafar TH, Stark PC, Schmid CH, Landa M, Maschio G, de Jong PE, et al. Progression of chronic kidney disease: the role of blood pressure control, proteinuria, and angiotensin-converting enzyme inhibition: a patient-level metaanalysis. Ann Intern Med. 2003;139:244-52.

407. Li Z, Lacson EJr., Lowrie EG, Ofsthun NJ, Kuhlmann MK, Lazarus JM, et al. The epidemiology of systolic blood pressure and death risk in hemodialysis patients. Am J Kidney Dis. 2006;48:606-15.

408. Mayer CC, Matschkal J, Sarafidis PA, Hagmair S, Lorenz G, Angermann S, et al. Association of ambulatory blood pressure with all-cause and cardiovascular mortality in hemodialysis patients: effects of heart failure and atrial fibrillation. J Am Soc Nephrol. 2018;29:2409-17.

409. Mark PB, Johnston N, Groenning BA, Foster JE, Blyth KG, Martin TN, et al. Redefinition of uremic cardiomyopathy by contrast-enhanced cardiac magnetic resonance imaging. KidneyInt. 2006;69:1839-45.

410. Wanner C, Krane V, Marz W, Olschewski M, Mann JF, Ruf G, et al. Atorvastatin in patients with type 2 diabetes mellitus undergoing hemodialysis. NEnglJ Med. 2005;353:238-48.

411. Weber T, Ammer M, Gunduz D, Bruckenberger P, Eber B, Wallner M. Association of increased arterial wave reflections with decline in renal function in chronic kidney disease stages 3 and 4. Am J Hypertens. 2011;24:762-9.

412. Agarwal R, SinhaAD, Pappas MK, Abraham TN, TegegneGG. Hypertension in hemodialysis patients treated with atenolol or lisinopril: a randomized controlled trial. Nephrol Dial Transplant. 2014;29:672-81.

413. Heerspink HJ, Ninomiya T, Zoungas S, de Zeeuw D, Grobbee DE, Jardine MJ, et al. Effect of lowering blood pressure on cardiovascular events and mortality in patients on dialysis: a systematic review and meta-analysis of randomised controlled trials. Lancet. 2009;373:1009-15.

414. Bansal N, McCulloch CE, Rahman M, Kusek JW, Anderson $\mathrm{AH}$, Xie D, et al. Blood pressure and risk of all-cause mortality in advanced chronic kidney disease and hemodialysis: the chronic renal insufficiency cohort study. Hypertension. 2015;65:93-100.
415. Pugh D, Gallacher PJ, Dhaun N. Management of hypertension in chronic kidney disease. Drugs. 2019;79:365-79.

416. Bhatnagar A, Pein U, Markau S, Weigand K, Fornara P, Girndt M, et al. Influence of SPRINT study type automated office blood pressure measurements on hypertension diagnosis in kidney transplant patients. Kidney Blood Press Res. 2018;43:310-7.

417. Mallamaci F, D’Arrigo G, Tripepi R, Leonardis D, Porto G, Testa A, et al. Office, standardized and 24-h ambulatory blood pressure and renal function loss in renal transplant patients. JHypertens. 2018;36:119-25.

418. Opelz G, Dohler B, Collaborative Transplant S. Improved long-term outcomes after renal transplantation associated with blood pressure control. Am J Transplant. 2005;5:2725-31.

419. Mange KC, Feldman HI, Joffe MM, Fa K, Bloom RD. Blood pressure and the survival of renal allografts from living donors. JAm Soc Nephrol. 2004;15:187-93.

420. Cross NB, Webster AC, Masson P, O'Connell PJ, Craig JC. Antihypertensives forkidney transplant recipients: systematic review and meta-analysis of randomized controlled trials. Transplantation. 2009;88:7-18.

421. Heinze G, Mitterbauer C, Regele H, Kramar R, Winkelmayer WC, Curhan GC, et al. Angiotensin-converting enzyme inhibitor or angiotensin II type 1 receptor antagonist therapy is associated with prolonged patient and graft survival after renal transplantation. J Am Soc Nephrol. 2006;17:889-99.

422. Pisano A, Bolignano D, Mallamaci F, D'Arrigo G, Halimi JM, Persu A, et al. Comparative effectiveness of different antihypertensive agents in kidney transplantation: a systematic review and meta-analysis. Nephrol Dial Transplant. 2019;pii:gfz092. https://doi.org/10.1093/ndt/gfz092.

423. Midtvedt K, Hartmann A, Foss A, Fauchald P, Nordal KP Rootwelt K, et al. Sustained improvement of renal graft function for two years in hypertensive renal transplant recipients treated with nifedipine as compared to lisinopril. Transplantation. 2001;72:1728-9.

424. Prischl FC, Wanner C. Renal outcomes of antidiabetic treatment options for type 2 diabetes-A proposed MARE definition. Kidney Int Rep. 2018;3:1030-8.

425. Molino C, Leite-Santos NC, Gabriel FC, Wainberg SK, Vasconcelos LP, Mantovani-Silva RA, et al. Factors associated with high-quality guidelines for the pharmacologic management of chronic diseases in primary care: a systematic review. JAMAIntern Med. 2019;179:553.

426. Leiba A, Fishman B, Twig G, Gilad D, Derazne E, Shamiss A, et al. Association of adolescent hypertension with future end-stage renal disease. JAMA Intern Med. 2019;179:517.

427. Feigin VL, Forouzanfar MH, Krishnamurthi R, Mensah GA, Connor M, Bennett DA, et al. Global burden of diseases I, risk factors $S$ and the GBDSEG. Global and regional burden of stroke during 1990-2010: findings from the global burden of disease study 2010. Lancet. 2014;383:245-54.

428. O’Donnell MJ, Xavier D, Liu L, Zhang H, Chin SL, RaoMelacini P, et al. Risk factors for ischaemic and intracerebral haemorrhagic stroke in 22 countries (the INTERSTROKE study): a case-control study. Lancet. 2010;376:112-23.

429. Pinna G, Pascale C, Fornengo P, Arras S, Piras C, Panzarasa P, et al. Hospital admissions for hypertensive crisis in the emergency departments: a large multicenter Italian study. Plos One. 2014;9:e93542.

430. Ahmed N, Nasman P, Wahlgren NG. Effect of intravenous nimodipine on blood pressure and outcome after acute stroke. Stroke. 2000;31:1250-5.

431. Liu S, Liu M, Peterson S, Miyake M, Vallyathan V, Liu KJ. Hydroxyl radical formation is greater in striatal core than in 
penumbra in a rat model of ischemic stroke. J Neurosci Res. 2003;71:882-8.

432. Jauch EC, Saver JL, Adams HP Jr., Bruno A, Connors JJ, Demaerschalk BM, et al. Guidelines for the early management of patients with acute ischemic stroke: a guideline for healthcare professionals from the American Heart Association/American Stroke Association. Stroke. 2013;44:870-947.

433. Lee M, Ovbiagele B, Hong KS, Wu YL, Lee JE, Rao NM, et al. Effect of blood pressure lowering in early ischemic stroke: meta-analysis. Stroke. 2015;46:1883-9.

434. Robinson TG, Potter JF, Ford GA, Bulpitt CJ, Chernova J, Jagger C, et al. Effects of antihypertensive treatment after acute stroke in the Continue or Stop Post-Stroke Antihypertensives Collaborative Study (COSSACS): a prospective, randomised, open, blinded-endpoint trial. lancet Neurol. 2010;9:767-75.

435. National Institute of Neurological D and Stroke rt PASSG. Tissue plasminogen activator for acute ischemic stroke. NEngl J Med. 1995;333:1581-7.

436. Ahmed N, Wahlgren N, Brainin M, Castillo J, Ford GA, Kaste M, et al. Relationship of blood pressure, antihypertensive therapy, and outcome in ischemic stroke treated with intravenous thrombolysis: retrospective analysis from Safe Implementation of Thrombolysis in Stroke-International Stroke Thrombolysis Register (SITS-ISTR). Stroke. 2009;40:2442-9.

437. Anderson CS, Huang Y, LindleyRI, ChenX, Arima H, Chen G, et al. Intensive blood pressure reduction with intravenous thrombolysis therapyfor acuteischaemicstroke(ENCHANTED): an international, randomised, open-label, blindedendpoint, phase 3 trial. Lancet 2019;393:877-88.

438. Potter JF, Robinson TG, Ford GA, Mistri A, James M, Chernova J, et al. Controlling hypertension and hypotension immediately post-stroke (CHHIPS): a randomised, placebo-controlled, double-blind pilot trial. lancet Neurol. 2009;8:48-56.

439. Sandset EC, Bath PM, Boysen G, Jatuzis D, Korv J, Luders S, et al. The angiotensin-receptor blocker candesartan for treatment of acute stroke (SCAST): a randomised, placebocontrolled, double-blind trial. Lancet. 2011;377:741-50.

440. Kim JY, Bae HJ. Spontaneous Intracerebral hemorrhage: management. JStroke. 2017;19:28-39.

441. Butcher KS, Jeerakathil T, Hill M, DemchukAM, Dowlatshahi D, Coutts SB, et al. The Intracerebral hemorrhage acutely decreasing arterial pressure trial. Stroke. 2013;44:620-6.

442. Anderson CS, HeeleyE, HuangY, WangJ, StapfC, DelcourtC, et al. Rapid blood-pressure lowering in patients with acute intracerebral hemorrhage. NEngl J Med. 2013;368:2355-65.

443. Manning L, Hirakawa Y, Arima H, Wang X, Chalmers J, Wang J, et al. Blood pressure variability and outcome after acute intracerebral haemorrhage: a post-hoc analysis of INTERACT2, a randomised controlled trial. lancet Neurol. 2014;13:364-73.

444. Qureshi AI, Palesch YY, Barsan WG, Hanley DF, Hsu CY, Martin RL, et al. Intensive blood-pressure lowering in patients with acute cerebral hemorrhage. N Engl J Med. 2016;375:1033-43.

445. SipahiI,SwaminathanA, NatesanV,DebanneSM, SimonDI, Fang JC. Effect of antihypertensive therapy on incident stroke in cohorts with prehypertensive blood pressure levels: a meta-analysis of randomized controlled trials. Stroke. 2012;43:432-40.

446. Kernan WN, Ovbiagele B, Black HR, Bravata DM, Chimowitz MI, Ezekowitz MD, et al. Guidelines for the prevention of stroke in patients with stroke and transient ischemic attack: a guideline for healthcare professionals from the
American Heart Association/American Stroke Association. Stroke. 2014;45:2160-236.

447. Dhamoon MS, Sciacca RR, Rundek T, Sacco RL, Elkind MS Recurrent strokeand cardiac risks afterfirstischemic stroke: the Northern Manhattan Study. Neurology. 2006;66:641-6.

448. PROGRESS Collaborative Group. Randomised trial of a perindopril-based blood-pressure-lowering regimen among 6,105 individuals with previous stroke or transient ischaemic attack. Lancet. 2001;358:1033-41.

449. Arima H, Chalmers J, Woodward M, Anderson C, Rodgers A, DavisS, etal.Lower targetblood pressures are safe and effective for the prevention of recurrent stroke: the PROGRESS trial. JHypertens. 2006;24:1201-8.

450. Liu L, Wang Z, Gong L, Zhang Y, Thijs L, Staessen JA, et al. Blood pressure reduction for the secondary prevention of stroke: a Chinese trial and a systematic review of the literature. Hypertens Res. 2009;32:1032-40.

451. OvbiageleB, DienerHC, YusufS, Martin RH, Cotton D, Vinisko R, et al. Level of systolic blood pressure within the normal range and risk of recurrent stroke. JAMA. 2011;306:2137-44.

452. SPS3 Study Group, Benavente OR, Coffey CS, Conwit R, Hart RG, McClure LA, et al. Blood-pressure targets in patients with recent lacunar stroke: theSPS3 randomised trial. Lancet. 2013;382:507-15.

453. Rothwell PM, Howard SC, Spence JD, Carotid Endarterectomy Trialists C. Relationship between blood pressure and stroke risk in patients with symptomatic carotid occlusive disease. Stroke. 2003;34:2583-90.

454. Turan TN, Cotsonis G, Lynn MJ, ChaturvediS, Chimowitz M, Warfarin-Aspirin Symptomatic Intracranial Disease Trial I. Relationshipbetweenblood pressureand strokerecurrence in patients with intracranial arterial stenosis. Circulation. 2007;115:2969-75.

455. Powers WJ, Clarke WR, Grubb RL Jr., Videen TO, Adams HP Jr., Derdeyn CP, et al. Lower stroke risk with lower blood pressure in hemodynamic cerebral ischemia. Neurology. 2014;82:1027-32.

456. Gottesman RF, Schneider AL, Zhou Y, Coresh J, Green E Gupta N, et al. Association between midlife vascular risk factors and estimated brain amyloid deposition. JAMA. 2017;317:1443-50.

457. Mortimer JA. The Nun Study: risk factors for pathology and clinical-pathologic correlations. Curr Alzheimer Res. 2012;9:621-7.

458. Emdin CA, Rothwell PM, Salimi-Khorshidi G, Kiran A, Conrad N, Callender T, et al. Blood pressure and risk of vascular dementia: evidence from a primary care registry and a cohort study of transient Ischemic attack and stroke. Stroke. 2016;47:1429-35.

459. Ninomiya T, Ohara T, Hirakawa Y, Yoshida D, Doi Y, Hata J, et al. Midlife and late-life blood pressure and dementia in Japanese elderly: the Hisayama study. Hypertension. 2011;58:22-8.

460. Oishi E, Ohara T, Sakata S, Fukuhara M, Hata J, Yoshida D, et al. Day-to-day blood pressure variability and risk of dementia in a general Japanese elderly population: the Hisayama study. Circulation. 2017;136:516-25.

461. Snyder HM, Corriveau RA, Craft S, Faber JE, Greenberg SM, Knopman D, et al. Vascular contributions to cognitive impairment and dementia including Alzheimer's disease. alzheimers Dement. 2015;11:710-7.

462. Yaffe K, Vittinghoff E, Pletcher MJ, Hoang TD, Launer LJ, Whitmer R, et al. Early adult to midlife cardiovascular risk factors and cognitive function. Circulation. 2014;129:1560-7.

463. SPRINT MIND Investigators for the SPRINT Research Group, Williamson JD, Pajewski NM, Auchus AP, Bryan 
RN, Chelune G, Cheung AK, et al. Effect of Intensive vs Standard Blood Pressure Control on Probable Dementia: A Randomized Clinical Trial. JAMA. 2019;321:553-561.

464. Gaillard R, Bakker R, Willemsen SP, Hofman A, Steegers EA, Jaddoe VW. Blood pressure tracking during pregnancy and the risk of gestational hypertensive disorders: the Generation R Study. Eur Heart J. 2011;32:3088-97.

465. Strevens H, Wide-Swensson D, Ingemarsson I. Blood pressure during pregnancy in a Swedish population; impact of parity. Acta Obstet Gynecol Scand. 2001;80:824-9.

466. Macdonald-Wallis C, Lawlor DA, Fraser A, May M, Nelson SM, Tilling K. Blood pressure change in normotensive, gestational hypertensive, preeclamptic, and essential hypertensive pregnancies. Hypertension. 2012;59:1241-8.

467. James PR, Nelson-Piercy C. Management of hypertension before, during, and after pregnancy. Heart. 2004;90:1499-504.

468. Wagner SJ, Barac S, Garovic VD. Hypertensive pregnancy disorders: current concepts. JClin Hypertens. 2007;9:560-6.

469. Bateman BT, Huybrechts KF, Fischer MA, SeelyEW, Ecker JL, Oberg AS, et al. Chronic hypertension in pregnancy and the risk of congenital malformations: a cohort study. Am J ObstetGynecol.2015;212:337e1-337e14.

470. Martin JA, Hamilton BE, Ventura SJ, Osterman MJ, Kirmeyer S, Mathews TJ, et al. Births: final data for 2009. Natl Vital Stat Rep. 2011;60:1-70.

471. Schutte JM, Steegers EA, Schuitemaker NW, Santema JG, de Boer K, Pel M, et al. Rise in maternal mortality in the Netherlands. BJOG. 2010;117:399-406.

472. Bramham K, Parnell B, Nelson-Piercy C, Seed PT, Poston L, Chappell LC. Chronic hypertension and pregnancy outcomes: systematic review and meta-analysis. BMJ. 2014;348:g2301.

473. Brown MA. Is there a role for ambulatory blood pressure monitoring in pregnancy? Clin Exp Pharmacol Physiol. 2014;41:16-21.

474. Brown MA, Lindheimer MD, de Swiet M, Van Assche A, Moutquin JM. The classification and diagnosis of the hypertensive disorders of pregnancy: statement from the International Society for the Study of Hypertension in Pregnancy (ISSHP). Hypertens Pregnancy. 2001;20:IX-XIV.

475. Working Group on High Blood Pressure in Pregnancy. Report of thenationalhigh blood pressure education program. AmJ Obstet Gynecol.2000;183:S1-S22.

476. Magee LA, Helewa M, Rey E; Hypertension Guideline Committee.Diagnosis, evaluation, and management of the hypertensive disorders of pregnancy. Strategic training initiative in research in the reproductive health sciences scholars. JObstet Gynaecol Can. 2008;30(3 Suppl):S1-S2.

477. Agrawal S, Cerdeira AS, Redman C, Vatish M. meta-analysis and systematic review to assess the role of soluble FMS-like tyrosinekinase- 1 and placentagrowth factor ratio in prediction of preeclampsia: the SaPPPhirE study. Hypertension. 2018;71:306-16.

478. Chappell LC, Enye S, Seed P, Briley AL, Poston L, Shennan $\mathrm{AH}$. Adverse perinatal outcomes and risk factors for preeclampsia in women with chronic hypertension: a prospective study. Hypertension. 2008;51:1002-9.

479. Tal R. The role of hypoxia and hypoxia-inducible factor-1alpha in preeclampsia pathogenesis. Biol Reprod. 2012;87:134.

480. National Institute for health and care Excellence (NICE). Hypertension in pregnancy: diagnosis and management. NICE guideline 2019, published 25 June 2019. www.nice. org.uk/guidance/ng133.

481. Regitz-Zagrosek V, Roos-Hesselink JW, Bauersachs J, Blomstrom-Lundqvist C, Cifkova R, De Bonis M, et al. 2018 ESC
Guidelines for the management of cardiovascular diseases during pregnancy. Eur Heart J. 2018;39:3165-241.

482. Rolnik DL, Wright D, Poon LC, O'Gorman N, Syngelaki A, de Paco Matallana C, et al. Aspirin versus placebo in pregnancies at high risk for preterm preeclampsia. NEngl J Med. 2017;377:613-22.

483. Brown MA, Buddle ML, Bennett M, Smith B, Morris R, Whitworth JA. Ambulatory blood pressure in pregnancy: comparison of the Spacelabs 90207 and Accutracker II monitors with intraarterial recordings. Am J Obstet Gynecol. 1995;173:218-23.

484. Lo C, Taylor RS, Gamble G, McCowan L, North RA. Use of automated home blood pressure monitoring in pregnancy: is it safe? Am J Obstet Gynecol. 2002;187:1321-8.

485. Natarajan P, Shennan AH, Penny J, Halligan AW, de Swiet M, Anthony J. Comparison of auscultatory and oscillometric automated blood pressure monitors in the setting of preeclampsia.Am J Obstet Gynecol. 1999;181:1203-10.

486. Bellomo G, Narducci PL, Rondoni F, Pastorelli G, StangoniG, AngeliG, etal.Prognostic value of 24-hourblood pressure in pregnancy. JAMA. 1999;282:1447-52.

487. Halligan A, O’Brien E, O'Malley K, Mee F, Atkins N, Conroy $\mathrm{R}$, et al. Twenty-four-hour ambulatory blood pressure measurement in a primigravid population. J Hypertens. 1993;11:869-73.

488. PennyJA, Halligan AW, Shennan AH, Lambert PC, Jones DR, de Swiet M, et al. Automated, ambulatory, or conventional blood pressure measurement in pregnancy: which is the better predictor of severe hypertension? Am J Obstet Gynecol. 1998;178:521-6.

489. Schmella MJ, Clifton RG, AlthouseAD, Roberts JM. Uric acid determination in gestational hypertension: is it as effective a delineator of risk as proteinuria in high-risk women? reprodSci. 2015;22:1212-9.

490. Abalos E, Duley L, Steyn DW, Gialdini C. Antihypertensive drug therapy for mild to moderate hypertension during pregnancy. Cochrane Database Syst Rev. 2018; https://doi. org/10.1002/14651858.CD002252.pub4.

491. Magee LA, von Dadelszen P, Rey E, Ross S, Asztalos E, Murphy KE, et al. Less-tight versus tight control of hypertension in pregnancy. NEngl J Med. 2015;372:407-17.

492. Magee LA, von Dadelszen P, Singer J, Lee T, Rey E, Ross S, et al. The CHIPS randomized controlled trial (control of hypertension in pregnancy study): is severe hypertension Just an elevated blood pressure? Hypertension. 2016;68:1153-9.

493. Magee LA, Abalos E, von Dadelszen P, Sibai B, Easterling T, Walkinshaw $S$, et al. How to manage hypertension in pregnancy effectively. Br JClin Pharmacol. 2011;72:394-401.

494. Nascimento SL, Surita FG, Cecatti JG. Physical exercise during pregnancy: a systematic review. curr Opin Obstet Gynecol. 2012;24:387-94.

495. Krysiak R, Samborek M, Stojko R. Primary aldosteronism in pregnancy. Acta Clin Belg. 2012;67:130-4.

496. Sibai BM, Grossman RA, Grossman HG. Effects of diuretics on plasma volume in pregnancies with long-term hypertension. Am J Obstet Gynecol. 1984;150:831-5.

497. Vadhera RB, Simon M. Hypertensive emergencies in pregnancy. Clin Obstet Gynecol. 2014;57:797-805.

498. Carles G, Helou J, Dallah F, Ibrahim N, Alassas N, Youssef M. Use of injectable urapidil in pregnancy-induced hypertension and preeclampsia. J Gynecol Obstet Biol Reprod. 2012;41:645-9.

499. Wacker JR, Wagner BK, Briese V, Schauf B, Heilmann L, Bartz C, etal. Antihypertensive therapy in patients with preeclampsia: a prospective randomised multicentre study comparing dihydralazine with urapidil. Eur J Obstet GynecolReprod Biol. 2006;127:160-5. 
500. Magee LA, Cham C, Waterman EJ, Ohlsson A, von Dadelszen P. Hydralazine for treatment of severe hypertension in pregnancy: meta-analysis. BMJ. 2003;327:955-60.

501. Mannisto T, Mendola P, Vaarasmaki M, Jarvelin MR, Hartikainen AL, Pouta A, et al. Elevated blood pressure in pregnancy and subsequent chronic disease risk. Circulation. 2013;127:681-90.

502. Chasan-Taber L, Willett WC, Manson JE, Spiegelman D, Hunter DJ, Curhan G, et al. Prospective study of oral contraceptives and hypertension among women in the United States. Circulation. 1996;94:483-9.

503. Lubianca JN, Moreira LB, Gus M, Fuchs FD. Stopping oral contraceptives: an effective blood pressure-lowering intervention in women with hypertension. J Hum Hypertens. 2005;19:451-5.

504. Mosca L, Benjamin EJ, Berra K, Bezanson JL, Dolor RJ, Lloyd-Jones DM, et al. Effectiveness-based guidelines for the prevention of cardiovascular disease in women-2011 update: a guideline from the American Heart Association. JAm Coll Cardiol. 2011;57:1404-23.

505. IssaZ, SeelyEW, RahmeM,FuleihanE-HG.Effects ofhormone therapy on blood pressure. Menopause. 2015;22:456-68.

506. LurbeE, Agabiti-Rosei E, CruickshankJK, DominiczakA, ErdineS, Hirth A, etal. 2016European Society of Hypertension guidelines for the management of high blood pressure in children and adolescents. J Hypertens. 2016;34:1887-920.

507. Chen X, Wang Y. Tracking of blood pressure from childhood to adulthood: a systematic review and meta-regression analysis. Circulation. 2008;117:3171-80.

508. Neuhauser HK, Thamm M, Ellert U, Hense HW, Rosario AS. Blood pressure percentiles by age and height from nonoverweight children and adolescents in Germany. Pediatr Electron Pages. 2011;127:e978-e88.

509. National High Blood Pressure Education Program Working Group on High Blood Pressure in Children and Adolescents. The fourth report on the diagnosis, evaluation, and treatment of high blood pressure in children and adolescents. Pediatrics. 2004;114:555-76.

510. Stergiou GS, Nasothimiou EG, Giovas PP, Rarra VC. Longterm reproducibility of home vs. office blood pressure in children and adolescents: the Arsakeion school study. Hypertens Res. 2009;32:311-5.

511. Wuhl E, Hadtstein C, Mehls O, Schaefer F, Escape Trial G. Home, clinic, and ambulatory blood pressure monitoring in children with chronic renal failure. Pediatr Res. 2004;55:492-7.

512. Chandar J, Zilleruelo G. Hypertensive crisis in children. Pediatr Nephrol. 2012;27:741-51.

513. Wuhl E, Witte K, Soergel M, Mehls O, Schaefer F, German Working Group on Pediatric H. Distribution of 24-h ambulatory blood pressure in children: normalized reference values and role of body dimensions. J Hypertens. 2002;20:1995-2007.

514. ESCAPE Trial group, Wuhl E, Trivelli A, Picca S, Litwin M, Peco-Antic A, et al. Strict blood-pressure control and progression of renal failure in children. N Engl J Med. 2009;361:1639-50.

515. Leyvraz M, Chatelan A, da Costa BR, Taffe P, Paradis G, Bovet $P$, et al. Sodium intake and blood pressure in children and adolescents: a systematic review and meta-analysis of experimental and observational studies. Int J Epidemiol. 2018;47:1796-810.

516. Williamson JD, Supiano MA, Applegate WB, Berlowitz DR, Campbell RC, Chertow GM, et al. Intensive vs standard blood pressure control and cardiovascular disease outcomes in adults aged $\rangle /=75$ years: a randomized clinical trial.JAMA. 2016;315:2673-82.
517. Bavishi C, Bangalore S, Messerli FH. Outcomes of intensive blood pressureloweringinolderhypertensive patients.JAm Coll Cardiol. 2017;69:486-93.

518. Warwick J, Falaschetti E, Rockwood K, Mitnitski A, Thijs L, Beckett N, et al. No evidence that frailty modifies the positive impact of antihypertensive treatment in very elderly people: an investigation of the impact of frailty upon treatment effect in the HYpertension in the Very Elderly Trial (HYVET) study, a double-blind, placebo-controlled study of antihypertensives in people with hypertension aged 80 and over. BMCMed. 2015;13:78.

519. Corrao G, Rea F, Monzio Compagnoni M, Merlino L, Mancia G. Protective effects of antihypertensive treatment in patients aged 85 years or older. J Hypertens. 2017;35:1432-41.

520. Watschinger B, Arbeiter K, Auer J, Drexel H, Eber B, Fasching P, et al. Klassifikation, Diagnostik und Therapie der arteriellen Hypertonie 2013: Empfehlungen der Österreichischen Gesellschaft für Hypertensiologie (ÖGH). Austrian J Hypert. 2013;17:99-108.

521. Wenzel RR, Slany J, Weber T, Zweiker R, Watschinger B. Hypertensiver Notfall - Hypertensive Entgleisung (Krise). Austrian J Hypert. 2014;18:97-100.

522. Perera GA. Hypertension; a manifestation of hypertensive vascular disease. NYState J Med. 1948;48:1724-8.

523. Vlcek M, Bur A, Woisetschlager C, Herkner H, Laggner AN, Hirschl MM. Association between hypertensive urgencies and subsequent cardiovascular events in patients with hypertension. J Hypertens. 2008;26:657-62.

524. Kaplan NM. Management of hypertensive emergencies. Lancet. 1994;344:1335-8.

525. Marik PE, Rivera R. Hypertensive emergencies: an update. Curr Opin Crit Care. 2011;17:569-80.

526. Rosei EA, Salvetti M, Farsang C. European Society of Hypertension Scientific Newsletter: treatment of hypertensive urgencies and emergencies. J Hypertens. 2006;24:2482-5.

527. Vaughan CJ, Delanty N. Hypertensive emergencies. Lancet. 2000;356:411-7.

528. Montgomery HE, Kiernan LA, Whitworth CE, Fleming S, Unger T, Gohlke P, et al. Inhibition of tissue angiotensin converting enzyme activity prevents malignant hypertension in TGR(mREN2)27. J Hypertens. 1998;16:635-43.

529. Strandgaard S, Paulson OB. Cerebral blood flow and its pathophysiology in hypertension. Am J Hypertens. 1989;2:486-92.

530. Novak V, Hajjar I. The relationship between blood pressure and cognitive function. Nat Rev Cardiol. 2010;7:686-98.

531. Nurnberger J, Keflioglu-Scheiber A, Opazo Saez AM, Wenzel RR, Philipp T, Schafers RF. Augmentation index is associated with cardiovascular risk. JHypertens. 2002;20:2407-14.

532. Shantsila A, Lane DA, Beevers DG, Lip GY. Lack of impact of pulse pressure on outcomes in patients with malignant phase hypertension: the West Birmingham Malignant Hypertension study. J Hypertens. 2012;30:974-9.

533. Weber T, Wassertheurer S. Moving on-on average in the right direction?: noninvasive methods to estimate central blood pressure. Hypertension. 2014;63:665-7.

534. Weber T, Wassertheurer S, Rammer M, Haiden A, Hametner B, Eber B. Wave reflections, assessed with a novel method for pulse wave separation, are associated with end-organ damage and clinical outcomes. Hypertension. 2012;60:534-41.

535. van den Born BH, Lip GYH, Brguljan-Hitij J, Cremer A, Segura J, Morales E, et al. ESC Council on hypertension position document on the management of hypertensive emergencies. Eur Heart JCardiovasc Pharmacother. 2019;5:37-46. 
536. Chester EM, Agamanolis DP, Banker BQ, Victor M. Hypertensive encephalopathy: a clinicopathologic study of 20 cases. Neurology. 1978;28:928-39.

537. Cremer A, Amraoui F, Lip GY, Morales E, Rubin S, Segura J, et al. From malignant hypertension to hypertensionMOD: a modern definition for an old but still dangerous emergency. J Hum Hypertens. 2016;30:463-6.

538. van den Born BJ, Lowenberg EC, van der Hoeven NV, de Laat B, Meijers JC, Levi M, et al. Endothelial dysfunction, platelet activation, thrombogenesis and fibrinolysis in patients with hypertensive crisis. J Hypertens. 2011;29:922-7.

539. Phillips SJ, Whisnant JP. Hypertension and the brain. The National High Blood Pressure Education Program. Arch Intern Med. 1992;152:938-45.

540. Houston MC. Nonsteroidal anti-inflammatory drugs and antihypertensives. Am J Med. 1991;90:42S-7S.

541. Houston MC, Weir M, Gray J, Ginsberg D, Szeto C, Kaihlenen PM, etal. The effects of nonsteroidal anti-inflammatory drugsonblood pressures of patients with hypertension controlled by verapamil. Arch Intern Med. 1995;155:1049-54.

542. Hirschl MM. Guidelines for the drug treatment of hypertensive crises. Drugs. 1995;50:991-1000.

543. Hirschl MM, Binder M, Bur A, Herkner H, Mullner M, Woisetschlager C, et al. Safety and efficacy of urapidil and sodium nitroprusside in the treatment of hypertensive emergencies. Intensive Care Med. 1997;23:885-8.

544. Task Force for the management of arterial hypertension of the European Society of Hypertension, Task Force for the management of arterial hypertension of the European Society of Cardiology. 2013 ESH/ESC Guidelines for the Management of Arterial Hypertension. blood Press. 2013;22:193-278.

545. Wacker J, Muller J, Grischke EM, Unkels R, Bastert G. Antihypertensive therapy in pregnancy-induced hypertension with urapidil.Zentralbl Gynakol. 1994;116:271-3.

546. Luscher TF, Wenzel RR, Noll G. Controversy over calcium antagonists: is there a rationally differentiating therapy? Dtsch Med Wochenschr. 1996;121:532-8.

547. Perez MI, Musini VM. Pharmacological interventions for hypertensive emergencies: a Cochrane systematic review. JHum Hypertens. 2008;22:596-607.

548. Furberg CD, Psaty BM. Corrections to the nifedipine metaanalysis. Circulation. 1996;93:1475-6.

549. Furberg CD, Psaty BM, Nifedipine MJV. Dose-related increase in mortality in patients with coronary heart disease. Circulation. 1995;92:1326-31.

550. Wenzel RR, Allegranza G, Binggeli C, Shaw S, Weidmann P, Luscher TF, et al. Differential activation of cardiac and peripheral sympathetic nervous system by nifedipine: role of pharmacokinetics. JAm Coll Cardiol. 1997;29:1607-14.

551. Wenzel RR, Bruck H, Noll G, Schafers RF, Daul AE, Philipp T. Antihypertensive drugs and the sympathetic nervous system. JCardiovasc Pharmacol. 2000;35:S43-S52.

552. Perl S, Riegelnik V, Mrak P, Ederer H, Rakovac I, Beck P, et al. Effects of a multifaceted educational program on blood pressure and cardiovascular risk in hypertensive patients: the Austrian herz.leben project. J Hypertens. 2011;29:2024-30.

553. Lane DA, Lip GY, Beevers DG. Improving survival of malignant hypertension patients over 40 years. Am J Hypertens. 2009;22:1199-204.

554. Kristensen SD, Knuuti J, Saraste A, Anker S, Botker HE, Hert SD, et al. 2014 ESC/ESA Guidelines on non-cardiac surgery: cardiovascular assessment and management: The Joint Task Force on non-cardiac surgery: cardiovascular assessment and management of the European Society of
Cardiology (ESC) and the European Society of Anaesthesiology (ESA). Eur HeartJ. 2014;35:2383-431.

555. Futier E, Lefrant JY, Guinot PG, Godet T, Lorne E, Cuvillon P, et al. Effect of individualized vs standard blood pressure management strategies on postoperative organ dysfunction among high-risk patients undergoing major surgery: a randomized clinical trial. JAMA. 2017;318:1346-57.

556. Blessberger H, Kammler J, Domanovits H, Schlager O, Wildner B, Azar D, et al. Perioperative beta-blockers for preventing surgery-related mortality and morbidity. Cochrane Database Syst Rev. 2018; https://doi.org/10.1002/14651858. CD004476.pub2.

557. Bouri S, Shun-Shin MJ, Cole GD, Mayet J, Francis DP. Metaanalysis of secure randomised controlled trials of betablockade to prevent perioperative death in non-cardiac surgery. Heart. 2014;100:456-64.

558. Rosenman DJ, McDonald FS, Ebbert JO, Erwin PJ, LaBella M, Montori VM. Clinical consequences of withholding versus administering renin-angiotensin-aldosterone system antagonists in the preoperative period. J Hosp Med. 2008;3:319-25.

559. Shammash JB, Trost JC, Gold JM, Berlin JA, Golden MA, KimmelSE. Perioperative beta-blockerwithdrawal and mortalityinvascularsurgical patients. AmHeartJ.2001;141:148-53.

560. Andersson C, Merie C, Jorgensen M, Gislason GH, TorpPedersen C, Overgaard C, et al. Association of beta-blocker therapy with risks of adverse cardiovascular events and deaths in patients with ischemic heart disease undergoing noncardiac surgery: a Danish nationwide cohort study. JAMA Intern Med. 2014;174:336-44.

561. Barrett TW, Mori M, De Boer D. Association of ambulatory use of statins and beta-blockers with long-term mortality after vascular surgery. J Hosp Med. 2007;2:241-52.

562. Hoeks SE, Scholte Op RWJ, van Urk H, Jorning PJ, Boersma E, Simoons ML, et al. Increase of 1-year mortality after perioperative beta-blocker withdrawal in endovascular and vascular surgery patients. Eur J Vasc Endovasc Surg. 2007;33:13-9.

563. London MJ, Hur K, Schwartz GG, Henderson WG. Association of perioperative beta-blockade with mortality and cardiovascular morbidity following major noncardiac surgery. JAMA. 2013;309:1704-13.

564. Turan A, You J, Shiba A, Kurz A, Saager L, Sessler DI. Angiotensin converting enzyme inhibitors are not associated with respiratory complications or mortality after noncardiac surgery. Anesth Analg. 2012;114:552-60.

565. Wallace AW, Au S, Cason BA. Association of the pattern of use of perioperative beta-blockade and postoperative mortality. Anesthesiology. 2010;113:794-805.

566. Zou Z, Yuan HB, Yang B, Xu F, Chen XY, Liu GJ, et al. Perioperative angiotensin-converting enzyme inhibitors or angiotensin II type 1 receptor blockers for preventing mortality and morbidity in adults. Cochrane Database Syst Rev. 2016; https://doi.org/10.1002/14651858.CD009210.pub2.

567. Roshanov PS, Rochwerg B, Patel A, Salehian O, Duceppe E, Belley-Cote EP, et al. Withholding versus continuing angiotensin-converting enzyme inhibitors or angiotensin II receptor blockers before Noncardiac surgery: an analysis of the vascular events in noncardiac surgery patIents cOhort evaluatioN prospective cohort. Anesthesiology. 2017;126:16-27.

568. Charlson ME, MacKenzie CR, Gold JP, Ales KL, Topkins M, Fairclough GP Jr., et al. The preoperative and intraoperative hemodynamic predictors of postoperative myocardial infarction or ischemia in patients undergoing noncardiac surgery. Ann Surg. 1989;210:637-48. 
569. Aronson S, Dyke CM, Stierer KA, Levy JH, Cheung AT, Lumb PD, et al. The ECLIPSE trials: comparative studies of clevidipine to nitroglycerin, sodium nitroprusside, and nicardipine for acute hypertension treatment in cardiac surgery patients. Anesth Analg. 2008;107:1110-21.

570. Cheung AT. Exploring an optimum intra/postoperative management strategy for acute hypertension in the cardiac surgery patient. JCard Surg. 2006;21(Suppl 1):S8-S14.

571. Dix P, Howell S. Survey of cancellation rate of hypertensive patients undergoing anaesthesia and elective surgery. Br J Anaesth. 2001;86:789-93.

572. Espinosa A, Ripolles-Melchor J, Casans-Frances R, AbadGurumeta A, Bergese SD, Zuleta-Alarcon A, et al. Perioperative use of Clevidipine: a systematic review and metaanalysis. Plos One. 2016;11:e150625.

573. Fleisher LA, Fleischmann KE, Auerbach AD, Barnason SA, Beckman JA, Bozkurt B, et al. 2014 ACC/AHA guideline on perioperative cardiovascular evaluation and management of patients undergoing noncardiac surgery: executive summary: a report of the American College of Cardiology/ American Heart Association Task Force on Practice Guidelines. Circulation. 2014;130:2215-45.

574. Haas CE, LeBlanc JM. Acute postoperative hypertension: a review of therapeutic options. Am J Health Syst Pharm. 2004;61:1661-73. quiz 1674-5.

575. Rimoldi SF, Messerli FH, Bangalore S, Scherrer U. Resistant hypertension: what the cardiologist needs to know. Eur Heart J. 2015;36:2686-95.

576. Calhoun DA, Jones D, Textor S, Goff DC, Murphy TP, Toto RD, et al. Resistant hypertension: diagnosis, evaluation, and treatment. A scientific statement from the American HeartAssociation ProfessionalEducation Committee of the Council for High Blood Pressure Research. Hypertension. 2008;51:1403-19.

577. Calhoun DA, Booth JN 3rd, Oparil S, Irvin MR, Shimbo D, Lackland DT, et al. Refractory hypertension: determination of prevalence, risk factors, and comorbidities in a large, population-based cohort. Hypertension. 2014;63:451-8.

578. PickeringTG, HallJE, Appel LJ, Falkner BE, Graves J, Hill MN, etal.Recommendationsforblood pressuremeasurementin humans and experimental animals: part 1: blood pressure measurement in humans: a statement for professionals from the Subcommittee of Professional and Public Education of the American Heart Association Council on High Blood Pressure Research. Circulation. 2005;111:697-716.

579. Jung O, Gechter JL, Wunder C, Paulke A, Bartel C, Geiger H, et al. Resistant hypertension? Assessment of adherence by toxicological urine analysis. J Hypertens. 2013;31:766-74.

580. LaurentS, CockcroftJ, VanBortelL, BoutouyrieP, Giannattasio C, Hayoz D, et al. Expert consensus document on arterial stiffness: methodological issues and clinical applications. Eur HeartJ. 2006;27:2588-605.

581. Zhao D, Liu H, Dong P, Zhao J. A meta-analysis of add-on use of spironolactone in patients with resistant hypertension. Int JCardiol. 2017;233:113-7.

582. DudenbostelT, CalhounDA. UseofAldosteroneantagonists for treatment of uncontrolled resistant hypertension. Am J Hypertens. 2017;30:103-9.

583. Eide IK, Torjesen PA, Drolsum A, Babovic A, Lilledahl NP. Low-renin status in therapy-resistant hypertension: a clue to efficient treatment. J Hypertens. 2004;22:2217-26.

584. Lohmeier TE, Iliescu R. The baroreflex as a long-term controller of arterial pressure. Physiology. 2015;30:148-58.

585. OttC, SchmidA, Toennes SW, DittingT, VeelkenR, Uder M, et al. Central pulse pressure predicts BP reduction after renal denervation in patients with treatment-resistant hypertension. EuroIntervention. 2015;11:110-6.
586. Ewen S, Ukena C, Linz D, Kindermann I, Cremers B, Laufs U, et al. Reduced effect of percutaneous renal denervation on blood pressure in patients with isolated systolic hypertension. Hypertension. 2015;65:193-9.

587. Daugherty SL, Powers JD, Magid DJ, Tavel HM, Masoudi FA, Margolis KL, et al. Incidence and prognosis of resistant hypertension in hypertensive patients. Circulation. 2012;125:1635-42.

588. Esler M, Krum H, Schlaich M, Schmieder R, Böhm M, SobotkaPA. Renalsympathetic denervationfor treatment of resistant hypertension: One year results from the Simplicity HTN-2 randomized controlled trial. J Am Coll Cardiol. 2012;59:E1705.

589. deLeeuwPW, BisognanoJD, Bakris GL, Nadim MK, HallerH, Kroon AA, et al. Sustained Reduction of Blood Pressure With Baroreceptor Activation Therapy: Results of the 6-Year Open Follow-Up. Hypertension. 2017;69:836-43.

590. Brandt MC, Mahfoud F, Reda S, Schirmer SH, Erdmann E Bohm M, et al. Renal sympathetic denervation reduces left ventricular hypertrophy and improves cardiac function in patients with resistant hypertension. J Am Coll Cardiol. 2012;59:901-9.

591. Bhatt DL, Kandzari DE, O’Neill WW, D'Agostino R, FlackJM, Katzen BT, et al. A controlled trial of renal denervation for resistant hypertension. N Engl J Med.2014;370:1393-401.

592. Mathiassen ON, Vase H, Bech JN, Christensen KL, Buus NH, Schroeder AP, et al. Renal denervation in treatment-resistant essential hypertension. A randomized, SHAM-controlled, double-blinded 24-h blood pressure-based trial. JHypertens. 2016;34:1639-47.

593. Kandzari DE, Bhatt DL, Brar S, Devireddy CM, Esler M, Fahy M, et al. Predictors of blood pressure response in the SYMPLICITYHTN-3 trial. Eur Heart J. 2015;36:219-27.

594. Fadl Elmula FE, Jin Y, Yang WY, Thijs L, Lu YC, Larstorp AC, et al. Meta-analysis of randomized controlled trials of renal denervation in treatment-resistant hypertension. blood Press. 2015;24:263-74.

595. Townsend RR, Mahfoud F, Kandzari DE, Kario K, Pocock S, Weber MA, et al. Catheter-based renal denervation in patients with uncontrolled hypertension in the absence of antihypertensive medications (SPYRAL HTN-OFF MED): a randomised, sham-controlled, proof-of-concept trial. Lancet. 2017;390:2160-70.

596. Kandzari DE, Bohm M, Mahfoud F, Townsend RR, Weber MA, Pocock S, et al. Effect of renal denervation on blood pressure in the presence of antihypertensive drugs: 6-month efficacy and safety results from the SPYRAL HTN-ON MED proof-of-concept randomised trial. Lancet. 2018;391:2346-55.

597. Zweiker D, Lambert T, Steinwender C, Weber T, Suppan M, Brussee $\mathrm{H}$, et al. Blood pressure changes after renal denervation are more pronounced in women and nondiabetic patients: findings from the Austrian Transcatheter Renal Denervation Registry. J Hypertens 2019;37:2290-7.

598. Azizi M, Schmieder RE, Mahfoud F, Weber MA, Daemen J, Lobo MD, et al. Six-month results of treatment-blinded medication titration for hypertension control following randomization to endovascular ultrasound renal denervation or a sham procedure in the RADIANCE-HTNSOLO trial.Circulation. 2019. https://doi.org/10.1161/CIRCULATIONAHA. 119.040451

599. Azizi M, Schmieder RE, Mahfoud F, Weber MA, Daemen J, DaviesJ, etal.Endovascularultrasound renaldenervation to treathypertension (RADIANCE-HTNSOLO): a multicentre, international, single-blind, randomised, sham-controlled trial. Lancet. 2018;391:2335-45. 
600. Naderi SH, Bestwick JP, Wald DS. Adherence to drugs that prevent cardiovascular disease: meta-analysis on 376,162 patients. Am J Med.2012;125:882-887e1.

601. Wang YR, Alexander GC, Stafford RS. Outpatient hypertension treatment, treatment intensification, and control in Western Europe and the United States. Arch Intern Med. 2007;167:141-7.

602. Corrao G, Parodi A, Nicotra F, Zambon A, Merlino L, Cesana G, etal. Better compliance to antihypertensive medicationsreduces cardiovascularrisk.JHypertens. 2011;29:610-8.

603. Tiffe T, Wagner M, Rucker V, Morbach C, Gelbrich G, Stork S, et al. Control of cardiovascular risk factors and its determinants in the general population-findings from the STAAB cohort study. BMC Cardiovasc Disord. 2017;17:276.

604. Gupta P, Patel P, Strauch B, Lai FY, AkbarovA, Gulsin GS, etal. Biochemical screening for nonadherence is associated with blood pressure reduction and improvement in adherence. Hypertension. 2017;70:1042-8.

605. Viigimaa M, Doumas M, Vlachopoulos C, Anyfanti P, Wolf J, Narkiewicz K, et al. Hypertension and sexual dysfunction: time to act. JHypertens. 2011;29:403-7.

606. Ibanez B, James S, Agewall S, Antunes MJ, BucciarelliDucci C, Bueno H, et al. 2017 ESC Guidelines for the management of acute myocardial infarction in patients presenting with ST-segment elevation: The Task Force for the management of acute myocardial infarction in patients presenting with ST-segment elevation of the European Society of Cardiology (ESC). eur HeartJ. 2018;39:119-77.

607. Fletcher BR, Hartmann-BoyceJ, HintonL, McManus RJ.The effect of self-monitoring of blood pressure on medication adherence and lifestyle factors: a systematic review and meta-analysis. Am J Hypertens. 2015;28:1209-21.

608. PrischlFC, RebhandlE, Kronberger M, KoppelstatterC. Successful communication with hypertensive patients [German]. JHyperton. 2018;22:29-34.

609. Johnson AG. NSAIDs and increased blood pressure. What is the clinical significance? drug Saf. 1997;17:277-89.

610. Armstrong EP, Malone DC. The impact of nonsteroidal antiinflammatory drugs on blood pressure, with an emphasis on newer agents. Clin Ther. 2003;25:1-18.

611. Frishman WH. Effects of nonsteroidal anti-inflammatory drug therapy on blood pressure and peripheral edema.Am J Cardiol.2002;89:18D-25D.

612. Ruschitzka F, Borer JS, Krum H, Flammer AJ, Yeomans ND, Libby P, et al. Differential blood pressure effects of ibuprofen, naproxen, and celecoxib in patients with arthritis: the PRECISION-ABPM (Prospective Randomized Evaluation of Celecoxib Integrated Safety Versus Ibuprofen or Naproxen Ambulatory Blood Pressure Measurement) Trial. Eur Heart J.2017;38:3282-92.

613. Solomon SD, Pfeffer MA, McMurray JJ, Fowler R, Finn P, Levin B, et al. Effect of celecoxib on cardiovascular events and blood pressure in two trials for the prevention of colorectal adenomas. Circulation. 2006;114:1028-35.

614. Chan CC, Reid CM, Aw TJ, Liew D, Haas SJ, Krum H. Do COX-2 inhibitors raise blood pressure more than nonselective NSAIDs and placebo? An updated meta-analysis. JHypertens. 2009;27:2332-41.

615. Krum H, Swergold G, Curtis SP, Kaur A, Wang H, Smugar SS, et al. Factors associated with blood pressure changes in patients receiving diclofenac or etoricoxib: results from the MEDAL study. J Hypertens. 2009;27:886-93.

616. Singh BK, Haque SE, Pillai KK. Assessment of nonsteroidal anti-inflammatory drug-induced cardiotoxicity. Expert Opin Drug Metab Toxicol. 2014;10:143-56.

617. Turtle EJ, Dear JW, Webb DJ. A systematic review of the effect of paracetamol on blood pressure in hypertensi- ve and non-hypertensive subjects. Br J Clin Pharmacol. 2013;75:1396-405.

618. Barton DA, Dawood T, Lambert EA, Esler MD, Haikerwal D, Brenchley C, et al. Sympathetic activity in major depressive disorder: identifying those at increased cardiac risk? JHypertens. 2007;25:2117-24.

619. Licht CM, de Geus EJ, SeldenrijkA, van Hout HP, Zitman FG, van Dyck R, et al. Depression is associated with decreased blood pressure, butantidepressantuse increases theriskfor hypertension. Hypertension. 2009;53:631-8.

620. Thase ME. Effects of venlafaxine on blood pressure: a metaanalysis of original data from 3744 depressed patients.jClin Psychiatry. 1998;59:502-8.

621. Dawood T, Lambert EA, Barton DA, Laude D, Elghozi JL, Esler MD, et al. Specific serotonin reuptake inhibition in major depressive disorder adversely affects novel markers of cardiac risk. Hypertens Res. 2007;30:285-93.

622. Schlaich MP, Lambert E, Kaye DM, Krozowski Z, Campbell DJ, Lambert G, et al. Sympathetic augmentation in hypertension: role of nerve firing, norepinephrine reuptake, and Angiotensin neuromodulation. Hypertension. 2004;43:169-75.

623. Boal AH, Smith DJ, McCallum L, Muir S, Touyz RM, Dominiczak AF, et al. Monotherapy with major antihypertensive drug classes and risk of hospital admissions for mood disorders. Hypertension. 2016;68:1132-8.

624. Cardoso F, Polonia J, Santos A, Silva-Carvalho J, Ferreira-de-Almeida J. Low-dose oral contraceptives and 24hour ambulatory blood pressure. Int J Gynaecol Obstet. 1997;59:237-43.

625. Oelkers W, Foidart JM, Dombrovicz N, Welter A, Heithecker R. Effects of a new oral contraceptive containing an antimineralocorticoid progestogen, drospirenone, on the renin-aldosterone system, body weight, blood pressure, glucose tolerance, and lipid metabolism. J Clin Endocrinol Metab. 1995;80:1816-21.

626. Hussain SF. Progestogen-only pills and high blood pressure: is there an association? A literature review. Contraception. 2004;69:89-97.

627. Cagnacci A, Zanin R, Napolitano A, Arangino S, Volpe A. Modification of 24-h ambulatory blood pressure and heart rate during contraception with the vaginal ring: a prospective study. Contraception. 2013;88:539-43.

628. Coylewright M, Reckelhoff JF, Ouyang P. Menopause and hypertension: an age-old debate. Hypertension. 2008;51:952-9.

629. Affinito P, Palomba S, Bonifacio M, Fontana D, Izzo R, Trimarco B, et al. Effects of hormonal replacement therapy in postmenopausal hypertensive patients. Maturitas. 2001;40:75-83.

630. Cifkova R, Hallen H. Cyclosporin-induced hypertension. JHypertens. 2001;19:2283-5.

631. Pesavento TE, Jones PA, Julian BA, Curtis JJ. Amlodipine increases cyclosporine levels in hypertensive renal transplant patients: results of a prospective study. J Am Soc Nephrol. 1996;7:831-5.

632. Beckey C, Lundy A, Lutfi N. Lercanidipine in the treatment of hypertension. Ann Pharmacother. 2007;41:465-73.

633. MadsenJK, JensenJD, JensenLW, PedersenEB. Pharmacokinetic interaction between cyclosporine and the dihydropyridine calcium antagonist felodipine. Eur J Clin Pharmacol. 1996;50:203-8.

634. Morales JM. Influence of the new immunosuppressive combinations on arterial hypertension after renal transplantation. Kidney IntSuppl.2002;(82):S81-7. 
635. Izzedine H, Ederhy S, Goldwasser F, Soria JC, Milano G, Cohen A, et al. Management of hypertension in angiogenesis inhibitor-treated patients. Ann Oncol.2009;20:807-15.

636. Zhu X, Wu S, Dahut WL, Parikh CR. Risks of proteinuria and hypertension with bevacizumab, an antibody against vascular endothelial growth factor: systematic review and meta-analysis. Am J Kidney Dis. 2007;49:186-93.

637. Escudier B, Eisen T, Stadler WM, Szczylik C, Oudard S, Siebels M, et al. Sorafenib in advanced clear-cell renal-cell carcinoma. NEnglJ Med. 2007;356:125-34.

638. Maitland ML, Kasza KE, Karrison T, Moshier K, Sit L, Black HR, et al. Ambulatory monitoring detects sorafenib-induced blood pressure elevations on the first day of treatment. Clin Cancer Res. 2009;15:6250-7.

639. ZhuX, Stergiopoulos K, Wu S. Riskof hypertension and renal dysfunction with an angiogenesis inhibitor sunitinib: systematic review and meta-analysis. Acta Oncol. 2009;48:9-17.

640. Bono P,ElfvingH,Utriainen T, OsterlundP, SaartoT,AlankoT, et al. Hypertension and clinical benefit of bevacizumab in the treatment of advanced renal cell carcinoma. Ann Oncol. 2009;20:393-4.

641. Wu S, Chen JJ, Kudelka A, Lu J, Zhu X. Incidence and risk of hypertension with sorafenib in patients with cancer: a systematic review and meta-analysis. Lancet Oncol. 2008;9:117-23.

642. Rixe O, Billemont B, Izzedine H. Hypertension as a predictive factor of Sunitinib activity. Ann Oncol. 2007;18:1117.

643. Molteni A, Heffelfinger S, Moulder JE, Uhal B, Castellani WJ. Potential deployment of angiotensin I converting enzyme inhibitors and of angiotensin II type 1 and type 2 receptor blockers in cancer chemotherapy. Anticancer Agents Med Chem. 2006;6:451-60.

644. Dirix LY, Maes H, Sweldens C. Treatment of arterial hypertension (AHT) associated with angiogenesis inhibitors. Ann Oncol.2007;18:1121-2.

645. Porta C, Paglino C, Imarisio I, Bonomi L. Uncovering Pandora's vase: the growing problem of new toxicities from novel anticancer agents. The case of sorafenib and sunitinib. Clin Exp Med. 2007;7:127-34.

646. Miura S, Fujino M, Matsuo Y, Tanigawa H, Saku K. Nifedipine-induced vascular endothelial growth factor secretion from coronary smooth muscle cells promotes endothelial tube formation via the kinase insert domain-containing receptor/fetal liver kinase-1/NO pathway. Hypertens Res. 2005;28:147-53.

647. Krapf R, Hulter HN. Arterial hypertension induced by erythropoietin and erythropoiesis-stimulating agents (ESA). Clin JAm Soc Nephrol. 2009;4:470-80.

648. Palmer SC, Saglimbene V, Mavridis D, Salanti G, Craig JC, Tonelli M, et al. Erythropoiesis-stimulating agents for anaemia in adults with chronic kidney disease: a network metaanalysis. Cochrane Database Syst Rev. 2014; https://doi. org/10.1002/14651858.CD010590.pub2.

649. Lee MS, Lee JS, Lee JY. Prevention of erythropoietin-associated hypertension. Hypertension. 2007;50:439-45.

650. Kaupke CJ, Kim S, Vaziri ND. Effect of erythrocyte mass on arterial blood pressure in dialysis patients receiving maintenance erythropoietin therapy. J Am Soc Nephrol. 1994;4:1874-8.

651. Santschi V, Chiolero A, Colosimo AL, Platt RW, Taffe P, Burnier M, et al. Improving blood pressure control through pharmacist interventions: a meta-analysis of randomized controlled trials. JAm Heart Assoc. 2014;3:e718.

652. Clark CE, Smith LF, Taylor RS, Campbell JL. Nurse led interventions toimprovecontrolofblood pressurein people with hypertension: systematic review and meta-analysis. BMJ.2010;341:c3995.

653. RuddP, HoustonmillerN, Kaufman J, KraemerH, BanduraA, Greenwald G, et al. Nurse management for hypertensionA systems approach. Am J Hypertens. 2004;17:921-7.

Hinweis des Verlags Der Verlag bleibt in Hinblick auf geografische Zuordnungen und Gebietsbezeichnungen in veröffentlichten Karten und Institutsadressen neutral. 